\title{
Pädagogen in der Politik. Netzwerke der Curriculumforschung in der Schweiz, 1968-1986
}

Höhener, Lukas

\begin{abstract}
Sind Jugendliche nach ihrer Schulzeit hinreichend auf die kommenden gesellschaftlichen Herausforderungen vorbereitet? Ist es an der Zeit, bestimmte Inhalte zugunsten anderer aus den Lehrplänen zu streichen? Und wer soll nach welchen Kriterien die neuen Lehrpläne entwickeln? Ab 1968 widmete sich die Curriculumforschung solchen Fragen und wurde zum prominenten Thema in Diskussionen von Erziehungswissenschaft, Bildungspolitik und Bildungspraxis. Lukas Höhener legt eine Analyse von lehrplanbezogenen Entwicklungen in der Schweiz zwischen 1968 und 1986 vor. Die umtriebigen, umsichtig geplanten Aktivitäten der von Karl Frey gegründeten Freiburger Arbeitsgruppe für Lehrplanforschung bilden den Ausgangspunkt, um Veränderungen in der Produktion von pädagogischem Wissen an Universitäten, aber auch in der Diffusion und Rezeption von Wissen in Bildungspolitik, -verwaltung und -praxis zu erläutern. So werden Entwicklungen in der Curriculumforschung, Praxisversuche mit Lernzielen in der Unterrichtsvorbereitung und die umfassenden Lehrplanreformen in den 1980er-Jahren in einen Zusammenhang gebracht. Historische Netzwerkanalysen machen zudem die Verflechtungen von Personen aus Forschung und Politik in Gremien und Kommissionen sichtbar. Sie fordern dazu auf, das Bedürfnis der Politik nach Expertise und Beratung als Folge von Reformdruck zu thematisieren. Zugleich geben sie Anlass zur Frage nach den Rückwirkungen politischer Interessen auf die thematische Ausrichtung der pädagogischen Forschung.
\end{abstract}

DOI: https://doi.org/10.33057/chronos.1625

Posted at the Zurich Open Repository and Archive, University of Zurich

ZORA URL: https://doi.org/10.5167/uzh-207834

Monograph

Published Version

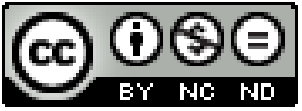

The following work is licensed under a Creative Commons: Attribution-NonCommercial-NoDerivatives 4.0 International (CC BY-NC-ND 4.0) License.

Originally published at:

Höhener, Lukas (2021). Pädagogen in der Politik. Netzwerke der Curriculumforschung in der Schweiz, 19681986. Zürich: Chronos.

DOI: https://doi.org/10.33057/chronos.1625 


\section{LUKAS HÖHENER}

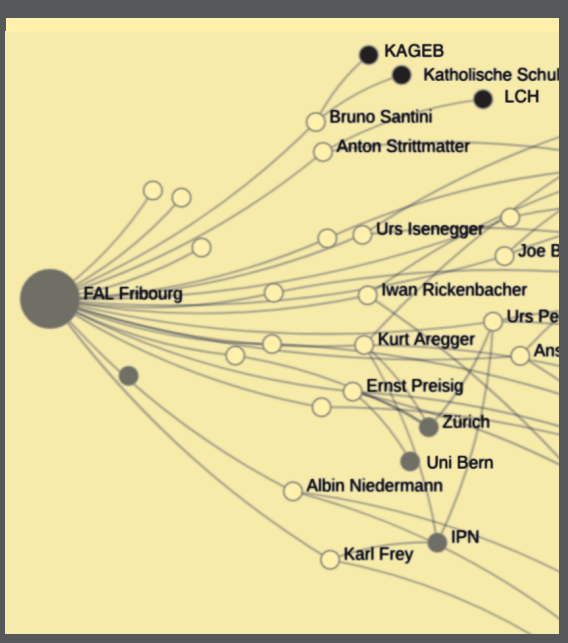

\section{IN DER \\ POLITIK}

PÄDAGOGEN

NETZWERKE DER

CURRICULUMFORSCHUNG

IN DER SCHWEIZ, 1968-1986

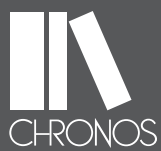


Historische Bildungsforschung

herausgegeben von

Patrick Bühler, Lucien Criblez,

Claudia Crotti und

Andreas Hoffmann-Ocon

Band 9 
Lukas Höhener

\section{Pädagogen in der Politik}

Netzwerke der Curriculumforschung in der Schweiz, 1968-1986 
Publiziert mit Unterstützung des Schweizerischen Nationalfonds zur Förderung der wissenschaftlichen Forschung.

\section{(C) $(1) \Theta$}

Informationen zum Verlagsprogramm:

www.chronos-verlag.ch

Umschlagbild: vgl. S. 66

(C) 202 I Chronos Verlag, Zürich

Print: ISBN 978-3-0340-I625-4

E-Book (PDF): DOI I0.33057/chronos.1625 


\section{Inhalt}

Vorwort des Herausgeberkreises

Vorwort

I Einleitung

2 Curriculumforschung in der Schweizvon der Entwicklung einer neuen Forschungsrichtung 29

2.I Curriculumbegriff und Forschungsstand 30

2.2 Förderung der Curriculumforschung in der Schweiz 36

2.2.I Ermittlung der dringlichen Forschungsbedürfnisse der Schweiz 36

2.2.2 Förderung der Curriculumforschung durch den 39 $\begin{array}{ll}\text { Schweizerischen Nationalfonds } & 39\end{array}$

2.3 Die Freiburger Arbeitsgruppe für Lehrplanforschung (FAL) 43

2.3.I Die Gründung der FAL 44

2.3.2 Institutionelle Verortung der FAL an der Universität Freiburg $\quad 46$

2.3.3 Der Forschungsgegenstand der FAL 48

2.3.4 Die Forschungspraxis der FAL $\quad 56$

2.4 Erklärungsansätze für die Einstellung der Curriculumforschung und der FAL

2.4.I Kaum Pädagogiklehrstühle für Forschende mit Schwerpunkt Curriculumforschung

2.4.2 Wirtschaftskrise und Stellenmangel in der Forschung 66

2.4.3 Bildungspolitisches Desinteresse und uneingelöste Versprechen 68

3 Vom wissenschaftlichen Programm zur politischen Debatte 7I

3.I Kontexte der Curriculumdebatten in den I970er-Jahren $\quad 72$

3.I.I Von der «äusseren» zur «inneren» Schulkoordination 72

3.I.2 Fortschrittsorientierung und Planungsgläubigkeit $\quad 74$

3.I.3 Verwendungsforschung und Bedarf an Expertise $\quad 76$

$\begin{array}{lll}3.2 & \text { Programmatik der Curriculumforschung } & 79\end{array}$

3.2.I Legitimationen von Curriculumreformen 79

3.2.2 Herausforderungen der Curriculumkonstruktion 82

3.2.3 Curriculumreform als Instrument der Schulreform 85

3.2.4 Leitideen der Öffentlichkeitsarbeit 87

3.2.5 Curriculumreform nach wissenschaftlichem Diktum 90

3.3 Das Curriculum in bildungspolitischen Debatten 9I

3.3.I Reformdebatten der Lehrerinnen- und Lehrerbildung 92

3.3.2 Formierungsdebatten der Erziehungs- und Bildungsforschung 96 
3.3.3 Bildungsplanung, Bildungsforschung und Schulpraxis im

Austausch

3.3.4 Umtriebige Vortragstätigkeit der FAL

3.3.5 Curricula als Dreh- und Angelpunkt

3.4 Wissenschaftler und Experten in Wissensnetzwerken

3.4.I Verflechtung und Dominanz der FAL in den Debatten

3.4.2 Wissenschaftler und Experten - Wissenschaftler als Experten

4 Lehrplanreformprojekte: Realisierung von Forschungskonzepten

4.I «Erziehungswissenschaftliche Kapazitäten» in

Lehrplanreformprojekten des Kantons Basel-Stadt

4.I.I Lehrplanreformen zur Umsetzung der Koedukation

4.I.2 Neue Prinzipien der Lehrplanarbeit ab i975

4.I.3 Das Institut für Unterrichtsfragen und Lehrerfortbildung (ULEF) I 24

4.I.4 Karl Frey in den Reformprojekten des Kantons Basel-Stadt I 28

4.I.5 Lehrplanreform im Kanton Basel-Stadt

4.2 Zentralschweizer Schulreformprojekte unter Anleitung des ZBS I32

4.2.I Oberstufenreform in der Zentralschweiz

4.2.2 Curriculumreform statt Lehrplanreform

4.2.3 Curriculumreform unter Anleitung des ZBS

4.2.4 Lehrplanreform in der IEDK

4.3 Das Freiburger EBAC-Projekt als «Erfahrungsfeld»

$\mathrm{I} 48$

4.3.I Beginn, Ziele und Organisation des EBAC-Projektes

4.3.2 «Demokratische Lehrplanarbeit»-

Herausforderungen und Widerstände

I 52

158

4.3.3 Das EBAC-Projekt als «Erfahrungsfeld» für die Forschung $\quad$ I62

4.3.4 Curriculum(theorie)entwicklungsprojekt in Freiburg $\quad$ I64

4.4 SIPRI - ein «Animationsprojekt» der EDK für die Primarschule I65

4.4.I Die Situation der Primarschulen ist zu überprüfen $\quad$ I66

4.4.2 Die Lernziele der Primarschule: Anspruch und Wirklichkeit $\quad$ I68

4.4.3 Lehrpläne und Lehrplanentwicklung im SIPRI-Teilprojekt I $\quad$ I7I

4.4.4 Verbesserung der Primarschullehrpläne $\quad$ I78

4.5 Planen, leiten und beraten: Bildungsengineering in Schulreformen $\quad$ I 79

$5 \quad$ Netzwerke und die Dynamisierung pädagogischer Forschung I85

Grafiken

Abkürzungen 


\section{Vorwort des Herausgeberkreises}

Im Vordergrund des vorliegenden Bandes steht die Curriculumforschung, -theorie und -entwicklung in den späten 1960er-, den 1970er- und frühen I980er-Jahren. Am Beispiel der deutschsprachigen Schweiz wird nicht nur ein bislang kaum erforschtes, zeitgeschichtliches Thema der Erziehungswissenschaft rekonstruiert, sondern auch danach gefragt, wie in diesem neuen Forschungsbereich erziehungswissenschaftliches Wissen produziert wurde, welche Strategien der Wissensdiffusion die untersuchte Freiburger Arbeitsgruppe für Lehrplanforschung (FAL) einsetzte und wie die Konzepte, Modelle, Befunde und Postulate in praktischen Zusammenhängen (Bildungspolitik/-verwaltung, Lehrerinnen- und Lehrerbildung, Schulen) genutzt wurden. Die Studie ist im Schnittfeld der Entwicklungen von Erziehungswissenschaft, Bildungspolitik und Schule und deren sich verändernden gegenseitigen Verhältnissen zu verorten und kann als wesentlicher Beitrag zu einer historischen Wissenschafts- und Wissenssoziologie der Erziehungswissenschaft in der Schweiz gelten.

Der bearbeitete Zeitraum, der zunächst von Bildungsplanung und Bildungs(planungs)euphorie, ab Mitte der I970er-Jahre aber zunehmend von Reformskepsis, Ernüchterung, ja Zukunftsängsten einer «verlorenen» Generation geprägt war, ist bildungshistorisch bislang noch wenig erforscht. Und das Thema der Curriculumforschung, -theorie und -entwicklung, das während des Bearbeitungszeitraum eines der meistdiskutierten Themen in Erziehungswissenschaft, Bildungspolitik und Bildungspraxis war, gehört bislang zu den Forschungsdesiderata der historischen Bildungsforschung - nicht nur für die Schweiz, sondern für den gesamten deutschsprachigen Raum. Für den Ausgangspunkt der Dissertation, die Aktivitäten der FAL der Universität Freiburg/Ue., liegen bislang überhaupt keine historischen Analysen vor, obwohl sie während eines Jahrzehnts die Diskussionen über die Curriculumentwicklung bestimmt und während eines weiteren Jahrzehnts die Lehrplanreformen in den Kantonen wesentlich beeinflusst hat.

Forschungsgegenstand im engeren Sinne sind die Akteure der FAL, deren Netzwerke und Akteursverflechtungen mit ausserwissenschaftlichen Reformakteuren sowie deren Tätigkeiten und Engagements in Wissenschaft, Politikberatung sowie Lehreraus- und -weiterbildung. Die so angelegten Analysen erlauben es dem Autor, Veränderungen sowohl in der Wissensproduktion (empirische Grundlagenstudien, Theorieproduktion, Modell- und Konzeptentwicklung) als auch im Wissenstransfer (Arbeitspapiere zuhanden von Bildungspolitik und Bildungsbehörden, Referate, Weiterbildung) und in der Wissensrezeption 
durch Bildungspolitik und Bildungspraxis zu beschreiben und vor dem Hintergrund der «Versozialwissenschaftlichung der Gesellschaft» (Weingart, I983) und der Etablierung eines wissenschaftlichen Expertentums auch im Bildungsbereich zu verorten. Gleichzeitig kann er aufzeigen, dass die Beratungs- und Entwicklungsangebote von Seiten der Wissenschaft in Bildungspolitik, Bildungsverwaltung und Lehrerbildung stark nachgefragt wurden. Die so angelegte Bildungsforschung war stark an Vewertungszusammenhängen orientiert. Dies führte einerseits zu einer «win-win»-Situation in Bildungsforschung und Praxisfeldern, tendierte aber andererseits auch dazu, deren Grenzen zu verwischen. Bildungsforschug wird dadurch zur Bildungspolitik - und kann an wissenschaftlicher Glaubwürdigkeit verlieren.

An vier Fallbeispielen werden die Forschungs- und Diffusionspraxen der FAL sowie deren Einfluss und Indienstnahme in praktischen Verwendungszusammenhängen verdeutlicht: Erstens wird gezeigt, wie die FAL die baselstädtische Lehrplanreform wesentlich beeinflusste. Zweitens wird der Einfluss der FAL am Beispiel der Zentralschweizer Oberstufenreform aufgezeigt. Gleichzeitig wird an diesem Beispiel der «Seitenwechsel» des Personals aus der FAL in die Bildungsverwaltung deutlich und damit der Wissenstransfer aus der Erziehungswissenschaft über den Stellenwechsel der Forschenden in die Bildungsadministration. Drittens wird am Beispiel des Freiburger LehrplanReformprojektes für die Primarschule gezeigt, wie die FAL-Mitglieder ihre theoretischen Konzepte und Konstrukte in einem grösseren Reformprojekt umsetzten. Gleichzeitig kann der Autor an diesem Fall auch die Rückwirkungen aus den Umsetzungsprojekten auf die ursprünglichen Curriculumkonzeptionen zeigen. Die Hierarchisierung der Lernziele bis auf die Ebene von Feinzielen, die Operationalisierung der Lernziele sowie deren Überprüfung folgen einem einfachen Regelzyklusmodell, dem ein technologisches Lernverständnis zugrunde liegt. Als letzter Fall wird das Lehrplan-Teilprojekt des von der Schweizerischen Konferenz der kantonalen Erziehungsdirektoren (EDK) initiierten SIPRI-Projekts (SIPRI: Überprüfung der Situation der Primarschulen; Laufzeit: I978-ı 986) analysiert. An diesem Projekt zeigt sich noch einmal, dass die Curriculumkonzepte der FAL nun Breitenwirkung entfalteten; sie dominierten die Diskussionen um die Lehrplanrevisionen der i $980 e r-$ und frühen I990er-Jahre ganz wesentlich.

Die FAL ist damit ein gutes Beispiel für die «Selbstermächtigung» der Wissenschaftler (nur Männer!) als Experten, deren Expertenwissen aber in Bildungspolitik und -verwaltung aufgrund der Komplexitätssteigerungen während der Bildungsexpansion und des Reformdrucks auch nachgefragt wurde.

Die vorliegende Studie gibt auch einen guten Einblick in disziplinäre Veränderungen. Sie ist sowohl bildungs- als auch wissenschaftshistorisch und gibt 
einen vertieften Einblick in die Veränderung des Verhältnisses zwischen der Produktion wissenschaftlichen Wissens und den entsprechenden Verwendungszusammenhängen. Sie zeigt auch auf, dass die Nutzenorientierung von Wissenschaft, die neuerdings immer wieder als Ökonomisierung beklagt wird, kein neues Phänomen darstellt. Dass damit Risiken verbunden sind, zeigt der Autor an der Auflösung der FAL Ende der i97oer-Jahre. Die Konzentration der wissenschaftlichen Disziplin auf Themen, die praktischen Nutzen versprechen, ist riskant, weil der Nutzen sich aus der Bearbeitung gesellschaftlicher Problemlagen ergibt, die sich aber verändern.

Patrick Bübler, Lucien Criblez, Claudia Crotti, Andreas Hoffmann-Ocon Muttenz, Solothurn und Zürich im Mai 202 I 


$$
\text { (name }
$$




\section{Vorwort}

Forschung ist etwas Gemeinschaftliches. Stunde um Stunde knien sich Forschende in Recherchen, Analysen und Lektüre, immer bemüht um ihr Erkenntnisinteresse und eine Schärfung ihrer Forschungsfragen. Mindestens so bedeutsam ist aber die Exposition der eigenen Ideen und Zwischenergebnisse im Feld. Für die Entwicklung dieser Arbeit waren die Teilnahme und der Austausch an Konferenzen unverzichtbar, ebenso die Rückmeldungen und Hinweise von Kolleginnen und Kollegen am Institut.

«Kommen in Ihrer Erzählung denn keine Frauen vor?» lautete eine der häufig gestellten Rückfragen nach meinen Referaten. Die Analysen von Dokumenten aus Forschungsgruppen, Kommissionen und politischen Gremien mussten mich diese Frage jeweils verneinen lassen. In den zwei Jahrzenten der I970erund 1980er-Jahre wurde die Aushandlung von pädagogischen Konzepten in der Lehrplanarbeit angeführt von Pädagogen und Politikern.

Bei der vorliegenden Arbeit handelt es sich um eine unwesentlich überarbeitete Fassung meiner Dissertationsschrift, welche im Rahmen meiner Anstellung am Institut für Erziehungswissenschaft der Universität Zürich entstanden ist und im Frühjahrssemester 2020 dort eingereicht wurde. Die Einbettung in ein anregendes und freundschaftliches Umfeld mit offenen Türen und Partizipationskultur habe ich als grosse Bereicherung erlebt.

Von ersten Skizzen bis zur mehrfach überarbeiteten Endversion meiner Arbeit habe ich immer wieder auf Unterstützung aus meinem Umfeld zählen dürfen. Für die Freiheit in der Bestimmung und Ausrichtung meines Forschungsthemas, für eine Betreuung nicht nur mit Hinweisen, sondern auch mit anregenden, weiterführenden Fragen und Ermutigungen, dafür und für einiges mehr gilt meinem Doktorvater Lucien Criblez mein besonderer Dank. Ich danke auch Philipp Gonon als Zweitbetreuer für seine fundierten Kommentare zu Referaten und Textentwürfen. Caroline Suter, Anja Giudici, Susanne Ender, Mirjam Staub und Michael Geiss verdanke ich wertvolle Rückmeldungen auf einzelne Kapitel. Thomas Ruoss und Karin Manz danke ich für die anregenden Hinweise vor allem in den Anfängen der Arbeit. Barbara Hof hat mich mit ihren präzisen Rückfragen und reichhaltigen Anmerkungen durch den gesamten Schaffensprozess begleitet. Vielen Dank, Barbara.

Meine Erhebung von Quellenmaterial wurde erheblich erleichtert durch die kompetente Unterstützung von Archivmitarbeitenden, insbesondere in den Staatsarchiven des Kantons Luzern, des Kantons Freiburg und des Kantons Basel-Stadt und im Archiv der Schweizerischen Koordinationsstelle für Bil- 
zurück dungsforschung. Dass mir warmherzig Zugang zum Privatarchiv von Karl Frey gewährt wurde, hat sich als Glücksfall herausgestellt. Und zu meiner grossen Freude haben sich einige ehemalige Mitglieder der Freiburger Arbeitsgruppe für Lehrplanforschung für Experteninterviews zur Verfügung gestellt. Sie erlaubten mir Einblicke in die Anfänge ihrer beruflichen Laufbahn, eine bewegte Zeit, über die so nirgends nachgelesen werden kann.

Der grösste Dank gilt meiner Partnerin und meinen Eltern für die unzähligen Momente der Unterstützung.

Winterthur, Januar 202 I 


\section{Einleitung}

Die Bestimmung dessen, was Schülerinnen und Schüler lernen sollen, wirft einige grundlegende Fragen auf, die immer wieder neu zu verhandeln sind: Bereitet das zu erwerbende Wissen genügend auf die kommenden gesellschaftlichen Herausforderungen vor? Sollte dieses Wissen nicht eher in Form von Kompetenzen vermittelt werden? Sind Lehrpläne (noch) das richtige Instrument zur Fixierung schulischen Wissens? Ist es an der Zeit, bestimmte Inhalte zugunsten anderer aus den Lehrplänen zu streichen? Und wer soll die neuen Lehrpläne entwickeln?

In den I970er-Jahren widmete sich unter dem Prädikat «Curriculumforschung» ein ganzer Forschungszweig ebensolchen Fragen. Die involvierten Forschenden waren bestrebt, die Neubestimmung der Inhalte von Lehrplänen der Volksschule mit wissenschaftlichen Methoden anzugehen. Die Ermittlung von Bildungsansprüchen sollte dem «Kampf geistiger Mächte» ${ }^{1}$ entzogen werden und neu in rationalisierter Form erfolgen. Dies galt auch für die Auswahl, Bewertung, Gewichtung, Operationalisierung und Überprüfung von Bildungsinhalten, welche neu als Bildungsziele zu formulieren waren. Für diesen fundamentalen Wandel im Bereich der Fixierung schulischen Wissens glaubten die Curriculumforschenden plausible theoretische Ansätze gefunden zu haben und auch mit eigenen Modellen und Konzepten begründen zu können, wie die dafür notwendigen Reformen zu planen und zu realisieren seien.

In der Schweiz leistete fast ausschliesslich die um 1968 von Karl Frey gegründete Freiburger Arbeitsgruppe für Lehrplanforschung (FAL) intensive Grundlagenforschung und Projektarbeit in diesem Bereich. Die Freiburger Arbeiten referierten ebenso wie vergleichbare Arbeiten in der Bundesrepublik Deutschland auf angelsächsische Konzepte der «curriculum studies», ohne sie sich vorbehaltlos zu eigen zu machen. Dass sich die Curriculumforschung als junger Zweig der eben im Entstehen begriffenen empirischen Bildungsforschung nicht längerfristig in der Wissenschaft etablierte, etwa durch entsprechend denominierte Professuren, tat der bildungspolitischen Rezeption ihrer Modelle und Konzepte bemerkenswerterweise keinen Abbruch. Insbesondere den pädagogischen Planungs- und Arbeitsstellen der Kantone galten die Arbeitsergebnisse als Bezugs- und Legitimationspunkt für die bald darauf flächendeckend einsetzenden Lehrplanreformen in der Schweiz. ${ }^{2}$

I Weniger, 1975 .

2 Gretler, 200I; Höhener, 20ı 8; Künzli, 2006a. 
Zeitgleich zu den universitären Forschungsbemühungen sind in der Schweizer Bildungspolitik Lehrplankoordination oder innere Schulreform zu Schlagworten der Reformprogramme geworden. ${ }^{3}$ Die Fragen zur Auswahl und Aufbereitung schulischer Inhalte folgten unmittelbar auf die laufenden Reformen zu schulischen Strukturen, etwa den Ausbau der obligatorischen Schulzeit oder diversen Gesamtschulversuchen. ${ }^{4}$ Für die neuen Schultypen oder zusätzlichen Schuljahre mussten schulische Inhalte bestimmt werden und die bestehenden Inhalte standen angesichts eines da und dort konstatierten Modernitätsrückstandes ebenso zur Disposition. ${ }^{5}$ Neue Konzepte der Lehrplankonstruktion waren bildungspolitisch erwünscht und nachgefragt, zumal sie in Aussicht stellten, zur Schulkoordination Hand zu bieten. ${ }^{6}$

An dieser Stelle setzt diese Arbeit an und fragt erstens nach der Produktion von Modellen und Konzepten der Schweizer Curriculumforschung, den Bezügen zu den internationalen «curriculum studies» und nimmt eine Bestimmung dieser neuen Wissensbestände vor. Es ist zweitens zu klären, wie dieses Wissen über die Neugestaltung von Lehrplänen und die dazu vorgelegten Verfahren in Debatten der Bildungspolitik rezipiert wurden. Drittens gilt es herauszuarbeiten, weshalb die Curriculumforschung während knapp zehn Jahren als bedeutender Teilbereich der aufstrebenden Bildungsforschung galt, in den frühen I980er-Jahren fast vollständig eingestellt wurde, ihre Forschungserzeugnisse aber in verschiedenen Bildungsreformprojekten zur leitenden Idee wurden. ${ }^{7}$ Schliesslich gilt das übergeordnete Forschungsinteresse den Bedingungen und Folgen von curriculumbasierten Modernisierungsprozessen vor dem Hintergrund wissenschaftlich legitimierter Gestaltungsabsichten und bildungspolitischer Wissensnachfrage. So analysiert dieses Projekt das wissensbasierte $\mathrm{Zu}$ sammenspiel von pädagogischer Forschung und bildungspolitischer Rezeption.

\section{Forschungsstand}

Die Frage nach dem Stellenwert pädagogischen Wissens in der Beziehung zwischen Forschung und Politik der Bildung ist bisher in erziehungswissenschaftlichen Studien unter verschiedenen Vorzeichen bearbeitet worden: Edwin Keiner meint sie in den späten I970er-Jahren vorrangig als «Theorie-PraxisProblem» angegangen zu sehen und betont zusätzlich den Bereich der Ver-

3 Aregger, Brunner und Isenegger, I972; Jenzer, Weiss und Strittmatter, 1978.

4 Bosche, 2013.

5 Heckhausen, 1970.

6 Criblez, 2007a.

7 Hameyer, 1992. 
wendungsforschung, welcher in den I990er-Jahren dann von der Forschung zu Aspekten des disziplinären Wissens in der Pädagogik abgelöst wurde. ${ }^{8}$ Klaus-Jürgen Tillmann zeichnet in seinen Beiträgen die Entwicklung des Verhältnisses von Erziehungswissenschaft und Bildungspolitik seit den I970erJahren für Deutschland nach, um letztlich zu argumentieren, dass die Prozesse und Mechanismen der Rezeption pädagogischer Forschungserkenntnisse beziehungsweise das «Herausfiltern» politikrelevanter Ergebnisse weitgehend ungeklärt sei. ${ }^{9}$ Radtke vertritt daran anschliessend die These, dass Forschungsresultate für andere gesellschaftliche Teilbereiche erst dann relevant seien, wenn sie Antworten auf Steuerungsfragen liefern würden, politisch durchsetzbar seien und eine Verbesserung der Arbeit in der Schulpraxis in Aussicht stellen würden. ${ }^{10}$ Und für das Verhältnis der pädagogischen Forschung zur Schulpraxis in den USA hat Jack Schneider herausgearbeitet, dass Lehrpersonen neue Ideen auf ihre Praxisrelevanz, den Komplexitätsgrad und die Kompatibilität mit bestehenden Vorstellungen abklopfen und gegebenenfalls ablehnen würden. Möglicherweise weil Austausch und Kooperation in Wechselspielen zwischen Gremien und Instanzen im Bildungswesen ordinär sind, ${ }^{\text {II }}$ werden sie oftmals nur mit pragmatischen Beschreibungen eingefangen. Niklas Luhmanns Griff zur soziologischen Grosstheorie hilft nur bedingt. Er thematisiert Austausch oberflächlich im Rahmen der Diskussion von strukturellen Kopplungen zwischen Teilbereichen wie Wissenschaft und Erziehung. ${ }^{12}$ Ewald Terhart benennt die Formate des Austausches unspezifisch als «Amt- und Dienstwege». ${ }^{13}$ Hingegen machen Stefan Hopmann und Rudolf Künzli mit Blick auf Entwicklungen in Deutschland geltend, dass jener Wissensaustausch, mindestens im Bereich der Lehrplanarbeit, die Folge einer institutionellen Segmentierung sei. Sie meinen damit ein «Netz von Mitwirkenden in Politik, Öffentlichkeit und Verwaltung, Lehreraus- und -fortbildung, Wissenschaft und Schule», welches an der Ausarbeitung neuer Lehrpläne am Werk sei, wobei nicht weiter auf dieses «Netz» eingegangen wird. ${ }^{14}$

Insgesamt wird deutlich, dass Wissenschaft und Politik im Bereich der Bildung in irgendeiner Form im Austausch sind und Wissen teilen, doch bleibt weitgehend unklar, nach welchen Verfahren und Prozessen sich die jeweiligen Felder Wissensbestände zu eigen machen und weshalb. Auch in den jüngst erstarkenden netzwerktheoretischen Ansätzen besteht Unklarheit «über Wissensver-

8 Keiner, 2002.

9 Tillmann, 2008a, S. 40. Vgl. dazu auch Tillmann, I99I; 2015.

Io Radtke, I996.

I I Oelkers, 2007.

I2 Luhmann, 20I5, S. $784 \mathrm{f}$.

I 3 Terhart, 200I, S. 27.

I4 Hopmann und Künzli, 20I s, S. 8. 
wendung und Wissensverarbeitung in Netzwerken». ${ }^{\text {Is }}$ Aufgrund dessen nimmt die vorliegende Arbeit eine Untersuchung der Produktion von pädagogischen Wissensbeständen im Bereich der Curriculumforschung aus den I970er-Jahren vor. Dabei ist nach Volker Roelcke zu bedenken, dass die systematisierte Einbeziehung und Reflexion der politischen Dimension bei der Produktion von neuem Wissen in den Wissenschaften für ein angemessenes Verständnis historischer Phänomene unverzichtbar sei. ${ }^{16}$ Indem die Forschungsperspektive dann erweitert wird um Analysen von ausserwissenschaftlichen Debatten über Curricula und verschiedenartige Rezeptionsprozesse in Bildungsreformprojekten mit einbezieht, leisten die vorliegenden Analysen einen Beitrag zur Erforschung der Beziehung zwischen universitärer Wissensproduktion und bildungspolitischer Wissensnutzung.

Der vorliegende Problemkomplex ist vor dem Hintergrund einiger zeitgeschichtlicher Kontexte einzuordnen, wobei das erst seit kurzem in den Fokus historischer Aufarbeitungen geratene Jahrzehnt der 1970er-Jahre nicht allzu einfach fassbar sei. Es entziehe sich einer eindeutigen Charakterisierung, markiere aber den Beginn eines fundamentalen Strukturwandels. ${ }^{17}$ Solche Veränderungen von wirtschaftlichen, sozialen, politischen oder mentalen Strukturen werden erstens den Nachwehen der 68er-Bewegungen zugeschrieben: Gesellschaftspolitische Lenkungs- und Entscheidungsmechanismen waren demnach zunehmend emanzipatorischen Ansprüchen und Experimenten unterworfen. Zweitens wird eine gesellschaftliche Ausdifferenzierung festgestellt, welche an einer zunehmenden Spezialisierung der Arbeitskräfte im Zuge des Übergangs zur Dienstleistungsgesellschaft abgelesen wird. ${ }^{18}$ Drittens werden die I970er-Jahre aus wirtschaftlicher Perspektive als Wendepunkt der Nachkriegsgeschichte betrachtet, zumal der Ölpreisschock dem seit Mitte der 1950er-Jahre andauernden goldenen Zeitalter ein Ende bereitete. ${ }^{19}$

Für die Bildungs- und Forschungslandschaft in der Schweiz blieben jene Entwicklungen nicht ohne Bedeutung. Bis die wirtschaftlich bedingte Zäsur in der Mitte der I $970 e r-J a h r e$ auch die Hochschulförderung erreichte und den stetigen Zuwachs von Forschungsgeldern des Bundes vorerst bremste, erfuhren Sozialwissenschaften wie die Politologie und die Soziologie einen stetigen Auf- und Ausbau. ${ }^{2 \circ}$ Währenddessen löste sich die pädagogische Forschung zunehmend von ihrer institutionellen Verortung in der Philosophie. Sie orientierte sich ver-

Keiner, 2002, S. 244.

Roelcke, 2010.

Jarausch, 2006.

Gilcher-Holtey, Eckert, François, Klessmann und Ruchniewicz, 2006.

Fabian, 2016.

Honegger, 2007. 
mehrt auch an Erkenntnissen ihrer Nachbardisziplinen und integrierte Theoreme aus der Psychologie, was wiederum zu intensiven Diskussionen über die Bezeichnung der eigenen Disziplin anregte. ${ }^{21}$ Mit dazu beigetragen hat die Tendenz, politische Entscheidungen vermehrt aufgrund «sozialwissenschaftlicher Interpretationsmuster» zu fällen. ${ }^{22}$ Es handelte sich dabei um einen Prozess, der als «Versozialwissenschaftlichung» in die Forschungsliteratur Einzug gehalten hat und meist verbunden wird mit der Herausbildung des Sozialstaates und dem Auftreten von Experten. ${ }^{23}$ Letztere sollten objektive, sozialwissenschaftliche Erkenntnisse einer «Zweck-Mittel-Rationalität» folgend übersetzen und auf entsprechende gesellschaftspolitische Problemstellungen anwenden. ${ }^{24}$ Der Bedarf an empirischen, sozialwissenschaftlichen Beschreibungen der «Wirklichkeit» im Bildungsbereich in den 1970er-Jahren war ferner gegeben durch Effekte der Bildungsexpansion: eine stetige Zunahme von Schülerinnen und Schülern infolge des «Babybooms», Erweiterungen der obligatorischen Schuldauer mit zusätzlichen Schuljahren sowie die Öffnung von Gymnasien und Universitäten für breitere Gesellschaftsschichten. ${ }^{25}$ Anlässlich der damit verbundenen Herausforderungen und mit dem Anspruch, zukünftige Entwicklungen errechnen und planen zu können, etablierte sich neben der an Universitäten verorteten pädagogischen Forschung die Bildungsforschung in Bildungsplanungsstellen. ${ }^{26}$ Diese meist an die kantonalen Bildungsverwaltungen gebundenen Planungsstellen legitimierten bildungspolitische und administrative Prozesse, produzierten und rezipierten Planungs- und Reformwissen und positionierten sich als Vermittlungsinstanz zwischen sozialwissenschaftlichem Wissen und dem Kontroll- und Entscheidungsbedürfnis politischer und administrativer Gremien. ${ }^{27}$ Obwohl im Zuge der Wirtschaftskrise gegenüber sozialwissenschaftlich abgestützten Steuerungsansprüchen erhebliche Skepsis laut wurde und Vorstellungen über die Planbarkeit des Fortschritts revidiert werden mussten, wurden Bildungsplanungsstellen bis in die I990er-Jahre schrittweise erweitert. ${ }^{28}$ Die Wege zwischen Forschung, Verwaltung und Anwendungsfeldern sozialwissenschaftlicher Forschung seien in der Phase des beschleunigten Wachstums mithin länger geworden.9

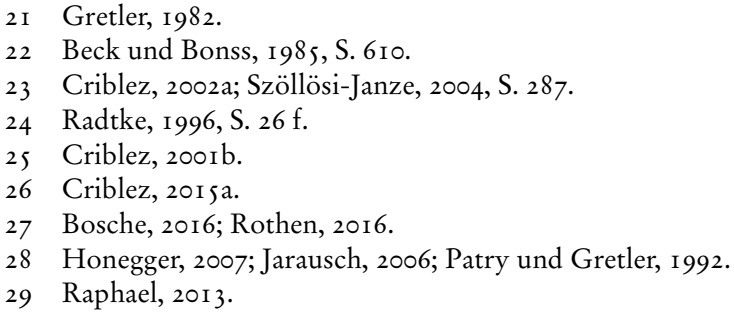




\section{Wissenschafts- und Wissensgeschichte als Heuristik}

Der Fokus auf die Produktion und den Austausch von Wissen zwischen Wissenschaft und Politik im Bereich der Bildung verlangt nach einer Rahmung, wie sie die Wissenschafts- und Wissensgeschichte anbietet. Wissensgeschichte betrachtet Wissen als historisches Phänomen und versucht zu ergründen, «wie, wann und gegebenenfalls warum ein bestimmtes Wissen auftaucht - und wieder verschwindet». ${ }^{\circ}$ Bei diesen Leitfragen ausgespart bleibt die Frage nach dem «wo», nach dem Ausgangspunkt von Wissen, weil die Existenz eines solchen abgelehnt wird und folglich unbestimmt bleiben müsse. Wissen entstehe hingegen «in einer kulturellen Produktion und in gesellschaftlichen Kräfteverhältnissen, für die - zum Beispiel - das Labor oder der Schreibtisch nur vorübergehende Kristallisationspunkte darstellen». ${ }^{\text {I }}$ Wer diesen momentanen Erscheinungspunkten folge, der könne die Bewegungen und Wandlungen von Wissen über nationalstaatliche, geografische und kulturelle Grenzen hinweg rekonstruieren..$^{32}$

Auch Prozesse der Wissensgenese oder die Einbindung wissenschaftlichen Wissens ins jeweilige Umfeld sind Gegenstand wissensgeschichtlicher Analysen. ${ }^{33}$ Daniel Speich Chassé und David Gugerli bemerken hierzu, dass die Wissensgeschichte eine kulturalistische Wende erlebt habe. ${ }^{34} \mathrm{Z}$ war liege der Fokus der jeweiligen Forschung nach wie vor auf der konkreten Herstellung von Fakten oder Denkweisen unter Berücksichtigung breiter soziopolitischer Konstellationen. Neu seien aber ebenso Fragestellungen zur Professionalisierung von Berufsgruppen, zur Popularisierung von Wissen oder zur Konstituierung von wissensgenerierenden Institutionen mit einzubeziehen. ${ }^{35}$ Demnach gilt es, die Prozesse der Wissenskonstruktion nicht losgelöst von den Bereichen der Anwendung dieses Wissens zu betrachten, oder, um mit Jakob Vogel zu präzisieren: Die Produktion von Wissen ist zeit- und kontextgebunden. ${ }^{6}$ Allein, der Schwierigkeit, «Wissen» eine abschliessende Definition zu unterlegen, vermag sich auch ein wissensgeschichtlicher Ansatz nicht zu entziehen und behilft sich hier mit einem "pluralisierten, von Wahrheit (und Wissenschaft) entkoppelten analytischen Begriff von Wissen» in der grundlegenden «Annahme von dessen sozialer Konstruiertheit». ${ }^{37}$

30 Sarasin, 20I I, S. I65.

3 I Sarasin und Kilcher, 20 I I, S. Io.

32 Ebd.

33 Breidbach, 2007.

34 Speich Chassé und Gugerli, 2012.

35 Vgl. dazu auch Hübinger, 2013; Stolleis, 2009; J. Vogel, 2004.

$36 \mathrm{~J}$. Vogel, 2004.

37 Behm, Drope, Glaser und Reh, 2017, S. 8. 
Versuche der in den I970er-Jahren vorherrschenden sozialwissenschaftlichen Verwendungsforschung, anhand von «mehr» und «besserem» Wissen eine überzeugendere Entscheidungsgrundlage für gesellschaftliche Handlungsfelder hervorzubringen, sind mittlerweile relativiert. Heiner Drerup begründet das damit, dass «die Wege wissenschaftlicher Informationen in der Wissenschaft, von der Wissenschaft in ausserwissenschaftliche Bereiche [...] vielfältiger und unübersichtlicher» seien..$^{38}$ Stattdessen stehen die Fragen im Raum, welche Informationen, Wissensprodukte, Modelle und Konzepte in bildungspolitischen Debatten überhaupt Beachtung fanden und welche Folgen die Institutionalisierung beispielsweise von Bildungsforschung auf ihre Verwendung hatte. ${ }^{39}$ Auf diesen für die Schweiz unbearbeiteten Bereich der Forschung deutet auch Lucien Criblez hin und betont seine Bedeutung für das Verhältnis von Bildungsforschung und Bildungspolitik. Er verweist überdies auf den Befund, wonach sozial- und erziehungswissenschaftliche Forschung «nicht nach den Regeln der Wissenschaft, sondern nach den Regeln der Bildungspolitik rezipiert» wurden..$^{\circ}$ Als Erklärung für diesen Sachverhalt sieht Werner Plumpe den gesellschaftlichen Wandel, welcher sich spätestens seit Mitte der I960er-Jahre derart komplex gestaltete, «dass die Politik den Überblick über Ausmass, Struktur und Richtung dieses Wandels verlor». ${ }^{41}$ Im Hintergrund steht zudem der Kommentar von Jakob Vogel zum Einfluss der Wissenssysteme auf verschiedene Praxisbereiche. Er betont, dass «die politischen, sozialen und kulturellen Einflüsse auf die Konstruktion des wissenschaftlichen Wissens in vielfältiger Weise ausgelotet wurden, [...] die gesellschaftlichen Wirkungen der auf diese Weise entstandenen Praktiken und Artefakte erstaunlich wenig erforscht wurden».42 Die eher wissenssoziologisch ausgerichtete Wissensgeschichte diskutiert die Entwicklungen ab der zweiten Hälfte des 20. Jahrhunderts unter dem Begriff Verwissenschaftlichung, welche an Veränderungen sowohl der Wissensproduktion als auch der Wissensnutzung abgelesen wird. Im Wandel der Wissensproduktion erkennt Holger Braun-Thürmann einen fortschreitenden Prozess der disziplinären Ausdifferenzierung, der neue Spezialgebiete und Disziplinen hervorgebracht habe. ${ }^{43}$ Weiter sei die Wissensproduktion zunehmenden bürokratischen Massgaben und entsprechenden Förderinstrumenten unterworfen worden und es habe eine zunehmende Kontextualisierung der Wissensproduktion stattgefunden. Braun-Thürmann meint damit eine Wechselwirkung der 
Wissenschaft mit anderen gesellschaftlichen Teilbereichen, wobei «relevante Forschungsbiete [...] als Reaktion auf gesellschaftliche Probleme begründet [werden], während das Praxisfeld wiederum zum Ort von wissenschaftlichen Entdeckungen wird».44

Darüber hinaus wird ein Wandel der Wissensnutzung beschrieben, welchen Lutz Raphael zu erkennen meint an einer «dauerhafte[n] Präsenz humanwissenschaftlicher Experten, ihrer Argumente und Forschungsergebnisse in Verwaltungen und Betrieben, in Parteien und Parlamenten».45 In der Absicht, die «spekulative» Gesellschaftstheorie zu verabschieden, setzte eine empirische Beobachtung, eine «Dauerreflexion» ein, geleistet von Expertengremien, Beiräten und Kommissionen. ${ }^{4}$ Peter Weingart bezeichnet dieses in den I960er- und I970er-Jahren vorherrschende Wissenschaftsverständnis als «technokratisch» und als Vorläufer eines späteren, skeptisch-konstruktivistischen Wissenschaftsverständnisses, ${ }^{47}$ «die naive Zuversicht jedenfalls, die in den I 960er Jahren bei der Verwissenschaftlichung Pate gestanden war, war Ende der I970er Jahre einer tiefgehenden «Ernüchterung〉 gewichen».$^{8}$

Ausgehend von den konstatierten Veränderungen muss sich nach Stefan Böschen der Fokus wissensgeschichtlicher Forschung auf Wissenskonflikte oder institutionelle Lösungsmechanismen richten, die aus der gegenseitigen Durchdringung von Wissenschaft und anderen gesellschaftlichen Feldern resultieren. ${ }^{49}$ Besonders aufgrund der Politisierung von Wissen seien bisweilen neue Arbeitsteilungen beobachtbar und letztlich veränderte Bedingungen der Erzeugung und Verteilung von Wissen relevant. Wissen wird ein regulierendes Moment zugeschrieben, einmal in Zirkulation, bleibt es nicht folgenlos..$^{\circ}$ Thomas Brandstetter verweist darüber hinaus auf die sozialen Beziehungen, welche die Zirkulation oder Überzeugungskraft von Wissen beeinflussen würden. ${ }^{\text {sI }}$ In dieselbe Richtung argumentiert auch Mitchell Ash in seinen Studien zur gegenseitigen Bezugnahme von Wissenschaft und Politik. Er vertritt die These, dass die Wissenschaft weit häufiger als die Politik die Initiative ergreife und ihre men argumentiert, basierend auf der Tatsache, dass Laien in bestimmten Bereichen die
Experten seien und deshalb eine Partizipation der Betroffenen notwendig sei. Weiterfüh-
rend Weingart, 2005. Plumpe, 2009, S. I I9.

49 Böschen, 2010.

50 Carrier, 2014. 5I Brandstetter, $20 \mathrm{I} 2$. 
Forschungsergebnisse wie auch personelle Ressourcen der Politik anbiete. Es seien deshalb etwaige Vernetzungen zwischen jenen gesellschaftlichen Teilsystemen in den Blick zu nehmen. ${ }^{52}$

Eine Analyse unter Berücksichtigung von Teilsystemen erfordert die Klärung des Umfelds der 1970er-Jahre, in welchem Entstehung, Diskussion und Rezeption von Wissen über theoretisch abgestützte Curricula vorangetrieben wurde. Ewald Terhart legt hierzu ein Modell vor und betont das komplexe Beziehungsgefüge zwischen Bildungsforschung, Bildungspolitik, Bildungsverwaltung und Bildungspraxis. ${ }^{53}$ Diese vier Bereiche seien streng formal betrachtet klar voneinander abzugrenzen, hinsichtlich bestimmter Orientierungen, Teilkulturen oder Interessen seien sie in sich aber je sehr heterogen aufgestellt. Ferner gebe es zwischen den vier Bereichen formalisierte Kommunikationskanäle. Gleichwohl müsse man «von einer starken wechselseitigen Durchdringung ausgehen».54

Der Bereich der Bildungspolitik ist nach Terhart bestimmt durch teilweise parteigebundene Interessen, das Streben nach dem Erfolg einmal ergriffener politischer Massnahmen sowie die Auswahl von zu bearbeitenden Problemen, auch Agenda-Setting genannt. Dem Bereich der Bildungsverwaltung schreibt er eine anhaltende, die Kontinuität wahrende Ordnungs- und Steuerungsfunktion zu. Dort werde das Wissen zur Abschätzung realistischer Reformmöglichkeiten gewahrt, weshalb diesem Bereich grosse Bedeutung beizumessen sei. Unter die Bildungspraxis subsumiert Terhart Handlungsprozesse in pädagogischen Einrichtungen, vorrangig unter Beteiligung von Lehrpersonen in Schulen. Die Bildungsforschung unterteilt Terhart letztlich in drei sehr unterschiedlich ausgerichtete Forschungstypen: erstens die problemerschliessende oder Grundlagenforschung, zweitens die Systembeobachtung, welche stärker mit Interessen der Bildungsverwaltung verknüpft sei, drittens die entwicklungsorientierte Forschung mit starken Bezügen zur Praxis und deren Einbezug in den Forschungsprozess.

Hinter der Einteilung des Bildungswesens in einzelne Teilbereiche verbirgt sich die Annahme, dass diese sich je über die ihnen eigenen Regulative und Strukturen bestimmen und so voneinander abgrenzen lassen. So erfolgt die Konstruktion und Rezeption von Wissen im Bereich der Bildungsverwaltung andersartig als etwa im Bereich der Bildungsforschung. Dieser Ansatz impliziert die Annahme von Feldern, wie sie Pierre Bourdieu in seinen Vorlesungen am

52 Ash, 2002.

53 Terhart, 200I. Zur Bestimmung von Bezugssystemen der Bildungsforschung haben Tillmann und Vollstädt mit denselben Bereichen gearbeitet, vgl. Tillmann und Vollstädt, $200 \mathrm{I}$.

Terhart, 200I, S. 27. 
Collège de France vertreten hat."s Anders als in funktionalistischen Theorien gesellschaftlicher Strukturen argumentiert Bourdieu mit geteilten Interessen von Akteuren an einem Gegenstandsbereich und spricht sich so aus für soziale Felder als hochdifferenzierte, thematisch gebundene, soziale Räume. ${ }^{6}$ Demnach sind die Rituale und Praktiken in der Erarbeitung wie im Umgang mit bereit gestellten Wissensbeständen feldspezifisch zu verstehen; da wird Wissen produziert, dort wird es rezipiert, adaptiert oder ignoriert, und zwar sowohl innerhalb eines Feldes wie auch Felder übergreifend. An der Verwendung des Begriffs Curriculum sollen der feldspezifische Umgang mit neuen Konzepten kurz illustriert und gleichsam dessen weitere Verwendung in dieser Arbeit erläutert werden.

Mitglieder der Freiburger Arbeitsgruppe betrieben Curriculumforschung als Angehörige der Universität Freiburg. Sie bezeichneten während ihrer gesamten Forschungstätigkeit als Curriculum, was bis anhin «Lehrplan» genannt worden war. ${ }^{57}$ Demgegenüber haben Akteure im Feld der Bildungspolitik diesen Begriff zur Benennung von Lehrplänen nie in ihr Vokabular übernommen, wenngleich sie sich in unterschiedlichen politischen Geschäften mit Curriculumforschung auseinandergesetzt haben. ${ }^{8}$ Demgemäss soll der offenbar feldspezifische Umgang mit Begriffen in der vorliegenden Arbeit sichtbar bleiben.

Eine zweite begriffliche Klärung muss an dieser Stelle noch zum Feld der Forschung angebracht werden. Im Bereich von Bildung und Erziehung wird Forschung als «Erziehungswissenschaft», «Bildungsforschung» oder «Pädagogik» charakterisiert, wobei die Differenzen der Begriffe tendenziell in Beiträgen zum Selbstverständnis der Disziplin betont werden. 59 Gemeinhin wird «Pädagogik» als Begriff mit grösserer Vorgeschichte genannt, wohingegen «Erziehungswissenschaft» mit der Weiterentwicklung und Ausdifferenzierung der Disziplin ab den I960er-Jahren in Verbindung gebracht wird und «Bildungsforschung» keine Verortung innerhalb der Disziplin voraussetzt. ${ }^{60}$ Nun experimentierten die Curriculumforscher in der Schweiz - es waren in der Scientific Community der Schweiz tatsächlich keine Curriculumforscherinnen aktiv - durchaus mit innovativen Forschungsmethoden und fühlten sich zeitweise kaum mehr einer geisteswissenschaftlichen Tradition verpflichtet. ${ }^{61}$ Indes waren sie eingebunden

Bourdieu, I993; 1997.

Rieger-Ladich, 2009, S. I 57.

Exemplarisch dazu Aregger, I97 Ib.

Jenzer et al., 1978.

K.-P. Horn, 2014; Tenorth, I986; 1990.

Criblez, 20r6b.

Vgl. dazu Kapitel 2. 
in ein Feld der szientistischen Bearbeitung von Fragestellungen des Pädagogischen, allerdings unter Rückgriff auf letztlich normative Auslegungen. ${ }^{62}$

\section{Zur Vorgehensweise}

Um wissensspezifische Entwicklungen im Wechselspiel von Pädagogik, Politik und Schule von den späten I960er-Jahren bis in die frühen I980er-Jahre methodisch fassbar zu machen, wird auf den Begriff Wissenskulturen zurückgegriffen, welcher auch als «epistemic communities» (EC) gefasst wird. Es handelt sich bei solchen «communities» um ein Professionellennetzwerk, geprägt von Schlüsselakteuren mit anerkannter Expertise und Kompetenz, nach Haas um einen Mechanismus, in welchem neue Ideen entstehen und zirkulieren. ${ }^{63} \mathrm{Pe}-$ ter Haas definiert eine EC anhand von vier Kriterien: Ihr wohnt erstens eine gemeinsame wertorientierende Grundüberzeugung inne, welche sich in der sozialen Wirkung ihrer Mitglieder äussert. Zweitens treffen Mitglieder einer EC Entscheidungen oftmals gemeinsam auf der Grundlage geteilter Wissensbestände, weshalb Erklärungen für das Verhalten von Mitgliedern aufgrund ihrer Zugehörigkeit zu einer EC zulässig sind. Drittens haben einer EC Angehörige gemeinsame Vorstellungen von intersubjektiver Gültigkeit und damit gemeinsame Kriterien der Wissensvalidierung, etwa bei der Differenzierung zwischen validem oder unsicherem Wissen bei Fragen der Lehrplankonstruktion. Viertens hat eine EC gemeinsame Veränderungsabsichten aus der Überzeugung heraus, eine Verbesserung der entsprechenden Problemlage zu erreichen.

Die Bedeutung von EC für die Erzeugung wie Verteilung von Wissen stützt sich ferner auf die wissenssoziologische Prämisse, wonach neben den methodologischen Standards soziale Faktoren innerhalb einer Fachgemeinschaft bei der Entwicklung und Bewertung der Gültigkeit von Theorien eine entscheidende Rolle spielen. ${ }^{64}$ Kontakt und Kommunikation unter Forschenden sind zentrale Elemente, die es zu untersuchen gilt, sofern man sich für Wissensentwicklung oder die Verbreitung von Wissen interessiert. ${ }^{65}$ Für jene Wissensdissemination kann Forschergruppen wie einzelnen Forschenden zwar bloss ein Teil der Verantwortung zukommen, da sie immer kontextgebunden arbeiten: «Nur das gesellschaftlich Akzeptierte wird zum Erfolg, und es ist evident, dass die Akzeptanz von wissenschaftsexternen Faktoren gesteuert wird.» ${ }^{66}$ Dass die

62 P. Vogel, I989.

63 Haas, I992; $201 \mathrm{I}$.

64 Schützeichel, $2007 \mathrm{~b}$.

65 Fangerau, 2009; Keller und Proferl, 2016.

66 Stolleis, 2009. 
Forschenden aber immer auch selbst zur Schaffung von Akzeptanz ihres Beitrags zur Wissenschaft und damit verbundener gesellschaftlicher Fragen beitragen, verhandelt und unterstreicht Weingart vor dem Hintergrund vorliegender Eigeninteressen der Wissenschaft. ${ }^{67}$ Peter Burke ergänzt, dass «informelle Netzwerke von führenden Persönlichkeiten und ihren Anhängern - ob sie sich nun an Universitäten, in Clubs oder Labors bilden - wesentlich für die Vermittlung von Wissen sind». ${ }^{6}$ Auf den Punkt bringt es Hübinger mit seinem Votum: «Die Erforschung wissenschaftlicher Tatsachen ist ohne die Erforschung der sie umfassenden Wissenschaftskulturen nicht zu haben.» ${ }^{69}$

Unter diesen Gesichtspunkten nimmt die vorliegende Arbeit eine Ergänzung beziehungsweise den teilweisen Ersatz der bisher verengenden Sicht auf Wissenschaft als System durch eine Vorstellung von Wissenschaft als Prozess vor. ${ }^{\circ}$ Es werden diejenigen Personen und Mittel fokussiert, welche Prozesse der wissenschaftlichen Erkenntnisgewinnung in Gang setzten, in Gang hielten und zielgerichtet ihre Resultate in ausseruniversitäre Zusammenhänge zu vermitteln versuchten. Das Projekt folgt ferner dem Ansatz, wonach Forschende wie die der FAL potenziell als Repräsentanten einer Wissenskultur eingeordnet werden können: Relevante Akteure, ihre Wissensordnungen und Praktiken werden rekonstruiert, ihr Mitwirken in Schul- und Curriculumprojekten wird ebenso analysiert wie deren Repräsentation in Zeitschriften, Monografien und Sammelbänden, um letztlich auch ihre Initiativen zur Wissensproduktion und -verbreitung herauszuarbeiten.

Zur Rekonstruktion und Visualisierung von Verflechtungen zwischen den Akteuren aus Forschung, Politik, Bildungsplanung und Schulpraxis wird auf Resultate einer umfangreichen historischen Netzwerkanalyse zurückgegriffen..$^{71}$ Generell tendieren Gremien mit strategischer Ausrichtung zur Unsichtbarkeit und sind gemeinhin auf der «Hinterbühne» angesiedelt. ${ }^{2}$ Gerade deshalb erschliesst sich eine umfassende Annäherung an Ausmass, Positionierung und Zugehörigkeit der involvierten Personen erst der historisch interessierten Forschung. Auch wenn über einzelne Formate des bildungspolitischen Austausches akribische Berichte oder Protokolle vorzufinden sind, bedarf es einer Ge-

67 Weingart, 2010.

68 Burke, 20I4, S. 289.

69 Hübinger, 2013 , S. 7.

70 Rheinberger, 2007; Rieger-Ladich, 2019.

7 I Die Abgrenzungen zwischen den Begriffen «Netzwerkforschung», «Netzwerkanalyse» und «Verflechtung» sind nicht abschliessend festgelegt. Sie sind symptomatisch für die je unterschiedlichen Forschungspraxen und Forschungskulturen in den Naturwissenschaften, den Sozialwissenschaften und der historischen Forschung. Weiterführend HoffmannOcon, 2013.

72 Goffman, 1991; Willems, 2010, S. 26r. 
samtschau unter Einbezug von Anstellungsverhältnissen, Projektengagements oder Tagungsaktivitäten, wie sie insbesondere die historische Netzwerkanalyse zu leisten vermag.

Die historischen Netzwerkanalysen fussen auf der Prämisse, wonach die Entstehung und Verbreitung von Wissen anhand der Beziehungen von Akteuren und ihrer Repräsentation in gesellschaftlichen Handlungsfeldern abgebildet und erklärt werden können. ${ }^{73}$ Nach diesem Verständnis und ergänzend zu den Ausführungen oben zu «epistemic communities» ist das Handeln von Akteuren eingebunden in soziale Beziehungen und soziale Kontexte und wird davon geprägt. ${ }^{74}$ So sind die sozialen Austauschprozesse wie auch die historisch bedingten Anlässe des Austausches in den Fokus zu nehmen, um die Entstehung und Verbreitung von Wissen greifbar zu machen.

Auf dieser Grundlage rekonstruiert die softwarebasierte Analyse zu Netzwerken der Curriculumforschung in der Schweiz quellenbasiert das Feld aller in der Schweiz tätigen Curriculumforscher und setzt es in Verbindung mit sämtlichen federführenden Akteuren in einschlägigen Bildungsdebatten an entsprechenden Anlässen des Austausches wie etwa Konferenzen und Seminaren.75 Zunächst ist also ein Wissenschaftsnetzwerk zu rekonstruieren, welches alle an der Curriculumforschung in der Schweiz beteiligten Forschenden und ihre gegenseitige Bezugnahme erfasst. Aufgrund der bisherigen Ausführungen ist anzunehmen, dass an der innerwissenschaftlichen Debatte vorrangig der FAL Zugehörige teilgenommen haben. In einem zweiten Schritt wird dann ein Netzwerk der an den bildungspolitischen Debatten aktiv Beteiligten aufgespannt. Daran werden nicht nur die besonders aktiven Personen in den Debatten und ihre Beziehungen untereinander ablesbar, sondern generelle Tendenzen zu Deutungshoheiten bestimmter Personen über die Debatten erkennbar. ${ }^{76}$ Ferner wird eine empirische Erklärungsgrundlage geschaffen, etwa für die Frage, weshalb Curricula als Forschungsgegenstand zum Thema langjähriger bildungspolitischer Debatten geworden sind.

Das Projekt stützt sich auf ein heterogenes Quellenkorpus. Es umfasst die bisher ungenutzten privaten Archivalien der Freiburger Arbeitsgruppe für Lehrplanforschung und Archivalien von dessen Gründer und anfänglichem Leiter Karl Frey. Darin sind Jahresberichte, Briefwechsel, Artikelentwürfe, Ordner zur Organisation von Tagungen oder Weiterbildungen enthalten. Zudem werden sämtliche spezifischen Monografien und Zeitschriftenartikel beigezogen, sowohl zur Rekonstruktion und Charakterisierung des wissenschaftlichen

73 Reitmayer und Marx, 2010.

74 H. Gruber und Rehrl, 2009; Jansen, 2003; Ketelhut, 2013.

75 Die Datenerhebung und -verarbeitung erfolgte in der Software Nodegoat.

76 Fuhse, 2019. 
Outputs der Curriculumforschung in der Schweiz als auch, um die Zusammenhänge und Querbezüge zu internationalen Debatten herauszuarbeiten. Zur Ergänzung der wissenschaftshistorischen Analysen wird ferner auf Berufungsakten der Universitäten Zürich und Freiburg (Schweiz) zurückgegriffen.

Für die rezeptionsgeschichtlichen Analysen von bildungspolitischen Debatten werden Bestände aus den Staatsarchiven der Kantone Basel-Stadt, Freiburg und Luzern beigezogen, wobei ebenfalls die Archivalien der Schweizerischen Konferenz der kantonalen Erziehungsdirektoren (EDK) aus dem Luzerner Staatsarchiv berücksichtigt werden. Das Quellenmaterial wird zusätzlich angereichert mit Archivalien aus zwei Bildungsplanungsstellen, dem Zentralschweizer Beratungsdienst für Schulfragen (ZBS) und der Schulberatungsstelle ULEF des Kantons Basel-Stadt. Schliesslich werden die Transkripte von Experteninterviews mit ehemaligen Mitgliedern der FAL und weiteren thematisch assoziierten Forschenden mit einbezogen. Sie dienen in einzelnen Fällen zur Validierung von Ergebnissen aus den Quellenanalysen.

Zur Analyse der Rezeption von wissenschaftlichen Forschungsleistungen in ausgewählten Bildungsreformprojekten werden vier Fallstudien vorgenommen. Sie konzentrieren sich I. auf den Kanton Basel-Stadt, 2. auf die Zentralschweizer Kantone mit einem Schwerpunkt beim Kanton Luzern, 3. auf den Kanton Freiburg, 4. auf ein mehrjähriges Grossprojekt der EDK mit dem Namen «Überprüfung der Situation der Primarschulen»(SIPRI). Diese vier Fallstudien machen je unterschiedliche Mechanismen der Ausbreitung und Rezeption von Wissen der Curriculumforschung in Bildungsreformprojekten greifbar. Erstens hat die FAL im Kanton Freiburg die Projektleitung wahrgenommen, zweitens haben im Kanton Basel-Stadt ausgewiesene Bildungsexperten ein Beratungsmandat übernommen, drittens wurde in der Zentralschweiz die Projektleitung einer Planungsstelle der Bildungsverwaltung übertragen und viertens wurde mit SIPRI ein Projekt mit zunächst ergebnisoffener Ausgangslage realisiert, welches bald Personal mit Erfahrung in Forschung zu und Entwicklung von Lehrplänen in einem seiner Teilprojekte zusammenführte.

Die Fallauswahl weist überdies auf eine Besonderheit des Netzwerks der Curriculumforschung hin: Die Bezüge der Curriculumforschung konzentrieren sich nahezu vollständig auf die deutschsprachige Schweiz. Davon zeugen auch die analysierten bildungspolitischen Debatten in der deutschsprachigen Lehrerinnen- und Lehrerpresse oder in Formaten zum Austausch von Akteuren aus Bildungsforschung, -administration und -praxis. Wenngleich die französischsprachige und die italienische Schweiz hie und da Bezugspunkte zu Entwicklungen in der Deutschschweiz aufweisen, verweist curriculumgeschichtliche Forschung auf sprachregionale Varietäten, die eine eigene Untersuchung wert wären. 


\section{Gliederung}

Die Entwicklungen der Curriculumforschung in der Schweiz und ihre Bedeutsamkeit für bildungspolitische Debatten und Reformprojekte ab den I970er-Jahren werden in vier Kapiteln aufgearbeitet, welche je mit einer Einleitung und einem Fazit abschliessen. Das folgende, zweite Kapitel ist zunächst geleitet von der Feststellung, dass die wissenschaftliche Auseinandersetzung mit Curricula in der Schweiz von umtriebigen Wissenschaftlern der FAL rund um Karl Frey angestossen wurde und zeitweise auf für diese Zeit ungewohnt umfangreiche finanzielle Förderung abgestützt war. Auch bedingt durch personelle Wechsel in der FAL und die Akquise neuer Forschungsprojekte veränderten sich die Forschungsschwerpunkte, gleichwohl überwogen die sozialwissenschaftlichen Zugänge und mithin Fragestellungen zur Überprüfbarkeit von schulischen Inhalten, Schulreformen oder Unterrichtsgewohnheiten von

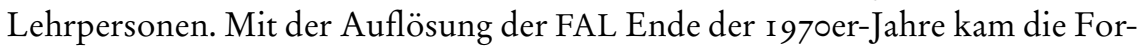
schung zu Curricula unter diesem Begriff in der Schweiz aber zum Stillstand. Schliesslich wird die These vertreten, dass die Hauptursache dafür nebst mangelnden Stellen im wissenschaftlichen Betrieb im nicht realisierbaren Bemühen der Curriculumforschung lag, eine wissenschaftlich begründete Setzung schulischer Inhalte in Form von Lernzielen vorzulegen.

Das dritte Kapitel nimmt eine fundierte Analyse von Debatten zu Curricula in ausserwissenschaftlichen Zusammenhängen vor und präsentiert zunächst drei bedeutende Kontexte: die Reformintentionen der EDK zur Koordination des Schulwesens über schulische Inhalte, die seit den I960er-Jahren verstärkte Tendenz zur Planung im Bildungswesen sowie ein damit verbundener Bedarf an Experten. Vor diesem Hintergrund arbeiten die Analysen das geteilte Verständnis der Schweizer Curriculumforscher als «epistemic community» heraus. Es wird aufgezeigt, dass die Gruppe mit einem Selbstverständnis als Bildungsexperten mittels breiter Informationskampagnen für ihre Konzepte zur erfolgreichen Bearbeitung der gegebenen bildungspolitischen Herausforderungen warb und in Reformen eine gestaltende Rolle einnahm. Die anschliessenden Untersuchungen von Reformdebatten aus der Lehrerinnen- und Lehrerbildung oder Anlässen zum Austausch von Personal aus Bildungsverwaltung, -forschung und -praxis weisen darauf hin, dass Konzepte der Curriculumforschung zum argumentativen Dreh- und Angelpunkt von Bildungsreformen avancierten, konzipiert als problemlösendes Instrument zur Koordination des kantonalen Volksschulwesens oder als Innovationswerkzeug zur Entwicklung von Schulen. Wie zu zeigen sein wird, ist dies nicht zuletzt einer personellen Verflechtung von Personal aus der Curriculumforschung mit Personal aus der Bildungsverwaltung geschuldet. Das 
Feld der Schlüsselakteure war überschaubar, und einige Mitglieder der FAL waren Teil davon.

Das letzte empirische Kapitel rollt den Untersuchungszeitraum der I970er-Jahre noch einmal auf, nun aber aus der Perspektive der Bildungspolitik. Dementsprechend wird die Rezeption curriculumspezifischer Wissensbestände in vier unterschiedlich gelagerten Bildungsreformprojekten in den Mittelpunkt gestellt. Die Auswertungen weisen deutlich darauf hin, dass in den Projekten zur Überarbeitung der Lehrpläne auf Konzepte der Curriculumforschung zurückgegriffen wurde, zumal durchgängig die schulischen Inhalte in Form von Lernzielen aufbereitet und die Ausarbeitung und Implementation von Leitideen in Lehrplänen verwirklicht wurde. Darüber hinaus zeigen die Analysen, dass in allen untersuchten Bildungsreformprojekten Personal mit Expertise beigezogen wurde. Die Experten brachten jeweils reflexartig Konzepte der Curriculumforschung ein und lancierten umfassende Reformen der Lehrpläne, selbst wenn zur Bearbeitung der Problemlagen andere Wege denkbar gewesen wären. Der Einbezug von Experten hatte in jedem Fall eine Komplexitätssteigerung der Verfahren, einen intensivierten Einbezug der Lehrpersonen in die Erarbeitungsprozesse der Lehrpläne sowie eine unerwartet lange Dauer der Verfahren zur Folge.

Die Schlussbetrachtungen bündeln die vorliegenden Analysen hinsichtlich des wissensbasierten Zusammenspiels von Forschung und Politik der Bildung. Aufgrund der vorliegenden Verflechtung von Entscheidungsträgern aus Forschung, Politik und Verwaltung zur Aushandlung von bildungspolitischen Themensetzungen in den 1970er- und frühen I980er-Jahren wird die folgende These vertreten: Netzwerke(r) der Curriculumforschung trugen massgeblich zur Dynamisierung der pädagogischen Forschung bei. Dieser Prozess veränderte das Verhältnis zwischen der pädagogischen Forschung und den in sie gesetzten bildungspolitischen Erwartungen und forcierte gleichsam eine Wende hin zur empirischen Bildungsforschung. 


\section{Curriculumforschung in der Schweiz - von der Entwicklung einer neuen Forschungsrichtung}

Theorien brauchen oder suchen Probleme. An der Sicht für die Probleme hat es der Curriculumforschung häufig gefehlt. ${ }^{\mathrm{I}}$

«Heute könnte man meinen, die Curriculumforschung sei dem Bewusstsein der Erziehungswissenschaftler weitgehend entrückt.» ${ }^{2} \mathrm{Zu}$ dieser Einschätzung gelangte Uwe Hameyer ${ }^{3}$ I 982 nach einer eingehenden Analyse der in der «Zeitschrift für Pädagogik» aufgeführten Dissertationen und Habilitationen sowie einer differenzierten Betrachtung der in der Bundesrepublik gesprochenen Fördergelder. Er konstatierte eine exponentielle Zunahme der Publikationen im Bereich der Curriculumforschung Anfang der I970er-Jahre, welcher ein massiver Rückgang der Publikationshäufigkeit zu diesem Thema gefolgt sei. Durchsucht man das bei Google Books hinterlegte deutschsprachige Textkorpus mithilfe des Ngram Viewer nach dem Schlagwort «Curriculum», bestätigt sich Hameyers Einschätzung, wie die Grafik I zu zeigen vermag.

Einer gaussschen Normalverteilung ähnlich schien der Begriff Curriculum in den deutschsprachigen Publikationen ab i965 aus dem Nichts aufzutauchen und seine Verwendung bis i975 massiv zuzunehmen. Danach flachte der Gebrauch des Begriffs fast ebenso schnell wieder ab.

Das vorliegende Kapitel nimmt diese Entwicklungen als Ausgangspunkt und sucht aus wissenschaftshistorischer Perspektive nach quellenbasierten Erklärungen für den scheinbar vorliegenden Aufstieg und Niedergang einer Forschungsrichtung, die in der pädagogischen Forschung der I970er-Jahre als Trend gegolten hat. ${ }^{4}$ Es ist erstens zu klären, inwieweit die hier angesprochenen Entwicklungen in der Forschungsförderung und in den pädagogischen Debatten in der Schweiz zu beobachten sind. Dabei wird fokussiert auf die einschlägige Grundlagenforschung. Ganz nach damaligem Verständnis des Schweizerischen Nationalfonds dienen wissenschaftliche Ergebnisse wie Dissertationen,

\footnotetext{
K. Frey, 1971, S. 53.

Hameyer, 1982, S. 8 I.

3 Uwe Hameyer hat zusammen mit Karl Frey und Henning Haft 1983 das bis heute jüngste deutschsprachige «Handbuch der Curriculumforschung. Übersichten zur Forschung I970-198 I» beim Beltz Verlag herausgegeben.

4 Gruber, 1992.
} 
Grafik 1: Verwendung des Schlagwortes «Curriculum» in deutschsprachiger Literatur 1960-1990

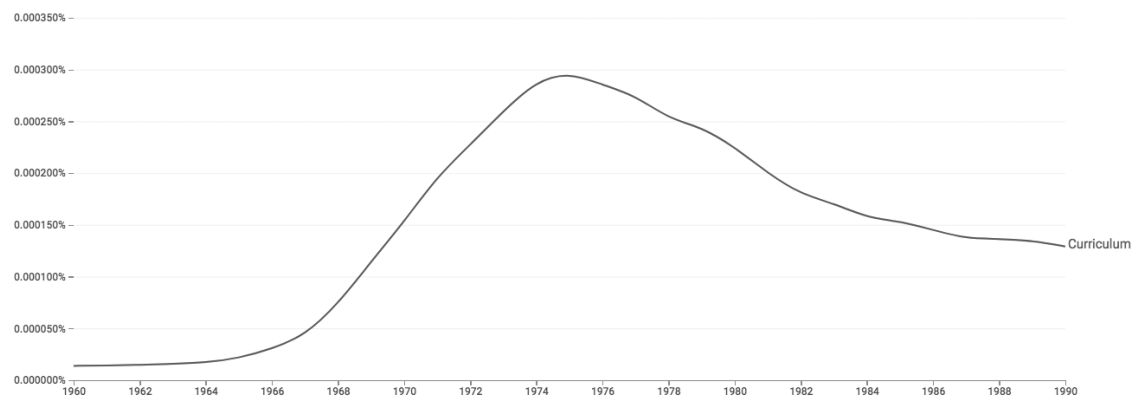

Quelle: Google Books, Ngram Viewer, abgerufen im April 2018

Habilitationen und vom Nationalfonds geförderte Projekte als Anknüpfungspunkte für die Analysen der Curriculumforschung in der Schweiz. Zweitens werden die Hauptakteure der Curriculumforschung in der Schweiz genannt, und deren Forschungsgegenstand und ihre Forschungspraxis werden analysiert. Zunächst folgen aber einige Einordnungen zum Begriff Curriculum und der Forschungsstand zur Curriculumforschung in der Schweiz wird dargelegt.

\subsection{Curriculumbegriff und Forschungsstand}

Im Februar 1970 veranstaltete die Freiburger Arbeitsgruppe für Lehrplanforschung (FAL) an der Universität Freiburg (Schweiz) eine zweitägige Konferenz mit dem Thema «Kriterien der Curriculumkonstruktion». Dieser Anlass wurde mit finanziellen Mitteln vom Schweizerischen Nationalfonds zur Förderung der wissenschaftlichen Forschung (SNF) unterstützt. Die Tagung führte Forschungsergebnisse aus Lernpsychologie, Entwicklungspsychologie und anderen Disziplinen zusammen mit dem Ziel, die Curriculumkonstruktion zu objektivieren und ihren praktischen Einsatz und die wissenschaftlichen Anforderungen dafür abzustecken.5 Im zugehörigen Tagungsband machten die Organisatoren einleitend deutlich, dass es sich beim Themenkomplex der Curri-

5 Auf der Liste der an der Tagung Referierenden stehen beispielsweise Hans Aebli, Jean Cardinet, August Flammer, Karl-Heinz Flechsig, Urs Haeberlin, Samuel Roller, Kurt Widmer oder Hans Wymann. 
culumkonstruktion um ein Hauptgebiet der aktuellen Curriculumforschung handle. ${ }^{6}$

Indes war die Forschung zur Konstruktion von Lehrplänen oder Lernzielen unter den Vorzeichen des Begriffs Curriculum in der Schweiz ein Bereich mit Bezügen nach Deutschland und in die USA. Dies wird im Folgenden anhand der Provenienz und Verwendung des Curriculumbegriffs aufgezeigt.

I959 veröffentlichte Josef Dolch seine viel zitierte Abhandlung zum «Lehrplan des Abendlandes. Zweieinhalb Jahrtausende seiner Geschichte». Darin zeigte er auf, dass bereits im Barock der Begriff Curriculum bei Morhofs «Polyhistor» geläufig gewesen sei, dann aber in Vergessenheit geraten sei. ${ }^{7}$ Für den Mitherausgeber des Handbuches für Curriculumforschung Uwe Hameyer nahm die Curriculumforschung ihren Anfang erst mit John Dewey zu Beginn des 20. Jahrhunderts in der «progressive education» in den USA und sie habe knappe 50 Jahre später mit Tyler und dessen «rationale» $^{8}$ eine theoretische Weiterentwicklung erfahren. ${ }^{9}$

Auf die angloamerikanische Herkunft machte auch Robinsohn in seiner für den deutschsprachigen Raum wegweisenden Veröffentlichung unter dem Titel «Bildungsreform als Revision des Curriculum» ${ }^{10}$ von 1967 aufmerksam, welche einen richtiggehenden Aufschwung der Forschung zu Curriculumthemen einleitete. ${ }^{\text {II }}$ Zur Verwendung dieses Terminus bemerkte er ferner, dass er «aus dem anglo-amerikanischen Gebrauch übernommen [wird], ohne dass damit eine Festlegung auf eine seiner spezifischen, unterschiedlichen Erscheinungsformen in England oder in den Vereinigten Staaten beabsichtigt ist». ${ }^{12}$

$\mathrm{Zu}$ Beginn der 1970er-Jahre schien in der deutschsprachigen Forschung weiterhin kein Konsens dazu erarbeitet worden zu sein, wofür der Begriff Curriculum stehen könnte. An einer Tagung mit dem Titel «Begriff und Funktion von Curricula», wiederum in Freiburg (Schweiz) und organisiert von der FAL, nahm Urs Isenegger eine Einordnung zweier damals vorherrschender Begriffsverständnisse vor. Bei einem Curriculum handle es sich entweder I. um «ein Dokument, als kodifizierte Form von Bildungsabsichten (in verschiedenen Variationen vom Lehrplan bisheriger Form, zu Lernzielkatalogen oder durchgeplanten Materialsystemen)», ${ }^{13}$ oder 2. "um ein Insgesamt von (geplanten) Aktivitäten, welche Unterricht beeinflussen (Lehrerberatung, Stundenpläne,

6 Lattmann, I97I, S. II.

7 Dolch, 1959, S. 319.

8 Tyler, I949.

9 Hameyer, 1983.

I0 Robinsohn, I967.

I I Göldi, 20I I; Hameyer, I983; Horlacher und De Vincenti, 20 I 4; Lattmann, 20 I 5.

I 2 Robinsohn, I967, S. I.

I3 Isenegger, I975, S. IO. 
Curricula im oben definierten Sinne, Lehrereinstellungen, Normvorstellungen innerhalb der Schule, vgl. dazu hidden curricula〉, <implizites Curriculum〉)». ${ }^{14}$ Die Tagungsteilnehmenden wurden darauf hingewiesen, dass die erste Definition die derzeit im deutschsprachigen Raum verwendete sei, während die zweite im angelsächsischen Raum die gebräuchliche sei. Ein Blick in die ersten einschlägigen Forschungsarbeiten aus der Schweiz verrät, dass dort der Begriff Curriculum von Beginn weg weitgehend mit «Lehrplan» gleichgesetzt wurde. Exemplarisch dazu nochmals Isenegger: «Der deutsche Ausdruck «Lehrplan» ist grundsätzlich gleichbedeutend wie 〈Curriculum〉. In der Vergangenheit wurden im deutschen Sprachraum z. T. beide Begriffe verwendet. Heute herrscht in der pädagogischen Fachsprache «Curriculum〉 vor. Diese Entwicklung wurde vor allem von S. B. Robinsohn mit seiner Schrift «Bildungsreform als Revision des Curriculum> beeinflusst.» ${ }^{\text {is }}$

Auf der Grundlage dieser Curriculumdefinition, die gleichsam für den weiteren Verlauf dieser Arbeit verwendet wird, erfolgt nun ein erster einordnender Überblick über die Curriculumforschung in der Schweiz.

Uwe Hameyer, Henning Haft und Karl Frey ${ }^{16}$ haben ${ }^{9} 8_{3}$ das bis heute jüngste deutschsprachige "Handbuch der Curriculumforschung» herausgegeben. ${ }^{17}$ Darin publizierte Armin Gretler, ${ }^{18}$ Direktor der Schweizerischen Koordinationsstelle für Bildungsforschung (SKBF), ${ }^{19}$ einen Artikel zur «Finanzierung der Curriculumforschung in der Schweiz». ${ }^{20}$ In seinen Ausführungen werden zwei Grundprobleme des hier untersuchten Gegenstandes manifest. Bei der Klassifizierung und Einordnung bildungsspezifischer Forschungsprojekte stellt sich

I4 Ebd.

is K. Frey, i969a, S. 270.

I6 Karl Frey (1942-2005) studierte Psychologie und Theologie in Löwen (B), Münster (D) und Freiburg $(\mathrm{CH})$, wo er in Pädagogik promovierte und 1968 die Freiburger Arbeitsgruppe für Lehrplanforschung am pädagogischen Institut gründete. I97 I folgte er dem Ruf auf eine ordentliche Professur nach Kiel und leitete dort das Institut für Pädagogik der Naturwissenschaften. Ab I 988 hatte er die Professur für Erziehungswissenschaft an der ETH in Zürich inne. Hintergrundinformationen zu einzelnen Personen werden hier und im Folgenden nur angeführt, wenn sie für den vorliegenden Forschungsgegenstand bedeutend sind.

I7 Hameyer, Frey und Haft, 1983.

I8 Armin Gretler war 197I-I999 Direktor der Schweizerischen Koordinationsstelle für Bildungsforschung. Zuvor studierte er Soziologie in Bern und war 1963-1971 freiberuflich in der Forschung und als Forschungsbeauftragter im Internationalen Arbeitsamt in Genf tätig.

I9 Die SKBF hat sich seit ihrer Gründung 1974 dem Ziel verschrieben, sämtliche Projekte zu Bildungsthemen in der Schweiz zu dokumentieren und in einer Datenbank aufzubereiten. Zur Entstehungsgeschichte weiterführend Grossenbacher und Oggenfuss, 20 I 5 . Bereits ein Jahr vor der eigentlichen Gründung der SKBF ist ein Tätigkeitsbericht der SKBF zu den Jahren I97I-I973 entstanden. Vgl. dazu Schweizerische Koordinationsstelle für Bildungsforschung, 1973 .

Gretler, 1983 . 
erstens die Frage: Welche Kriterien wurden herangezogen, um ein Projekt der Curriculumforschung zuzurechnen? Und im Anschluss daran wäre zu klären, wie Projekte der Curriculumforschung von Projekten der Curriculumentwicklung abgegrenzt werden können.

Armin Gretler wies sämtliche Curriculumprojekte entweder der Kategorie «Curriculumforschung im engeren Sinne» oder "Curriculumforschung im weiteren Sinne» zu, wobei die offenere Kategorie wie folgt umrissen wurde: «Der Bereich Curriculumforschung i. w. S. wurde hier weitestmöglich interpretiert und umfasst Grundlagenforschung in folgenden Bereichen: Entwicklungspsychologie, Lernpsychologie, Kognitionspsychologie, pädagogische Psychologie, Didaktik und einen Teil der Forschung von und um Jean Piaget. ${ }^{21}$ Diese Definition geht offensichtlich weit über Iseneggers Verständnis von Curriculumforschung hinaus. Sie erlaubt hingegen erstens eine inhaltliche Annäherung an den Gegenstand jenes Forschungsbereichs und zeigt die entsprechenden Bezugsdisziplinen auf. Eine Melange von psychologischen und pädagogischen Fragestellungen wurde zur Ausgangslage für theoretisch abgestützte Überlegungen im Lehrplanbereich und konkretisiert anhand von Lernzielen, Leitideen oder in didaktischen Konzepten zuhanden der Lehrpersonen. Curriculumforschende waren davon überzeugt, eine wissenschaftlich verortete Neuerung des Bildungswesens anzustossen und anhand von Lehrplänen zu realisieren. ${ }^{22}$ Die Ermittlung von Bildungsansprüchen sowie die Auswahl, Bewertung, Gewichtung, Operationalisierung und Überprüfung von Bildungszielen und -inhalten sollten dem «Kampf geistiger Mächte» ${ }^{23}$ entzogen und in rationalisierter Form unter wissenschaftlicher Anleitung vorgenommen werden, zumal die bisherige geisteswissenschaftliche Reflexionsarbeit zu Lehrplänen für die weitere Planung und Revision als untauglich erachtet wurde. ${ }^{24}$

Die Kategorisierungspraxis von Armin Gretler provoziert zweitens die Frage nach der Abgrenzung von Projekten der Curriculumforschung von Projekten der Curriculumentwicklung. Gretlers Erhebungspraxis bei der SKBF hat hier$\mathrm{zu}$ keine Unterscheidung vorgesehen. Ob es sich bei den kategorisierten Bildungsforschungsprojekten um eine theorieorientierte Habilitationsschrift im Curriculumbereich oder um ein Lehrplanentwicklungsprojekt für die Volksschule einer kantonalen Bildungsplanungsstelle gehandelt hat, war in seiner Zählweise einerlei. ${ }^{25}$

2 I Nezel und Ghisla, 1977, S. 86.

22 Flitner, I977; Knab, I969; Künzli und Frey, I977; Oswald, I984; Robinsohn, I97 I.

23 Weniger, 1975, S. 216.

24 Achtenhagen und Meyer, i97I.

25 Die von Gretler erhobenen Daten vermögen generell einen guten ersten Überblick über das Feld der Bildungsforschung zu geben, für systematische Analysen sind die zugrunde liegenden Kategorisierungen aus den genannten Gründen problematisch. 
Für das «Handbuch der Curriculumforschung» von 1983 ist ein zweiter einordnender Artikel von Kurt Aregger und Armin Gretler zu Forschungsleistungen in der Schweiz in jenem Bereich verfasst worden. ${ }^{26}$ Die beiden Autoren kamen darin zum Schluss, dass auf eine chronologische Darstellung der Curriculumforschung der Schweizer I970er-Jahre verzichtet werden müsse, dass sie mehr als eines Erzählstranges bedürfe, es sei denn, man entschliesse sich zur Lückenhaftigkeit. Allerdings hielten sich ihre Ausführungen weniger lange bei der eigentlichen Forschung als bei deren Rezeption in Form von Projekten in der Praxis auf.

Deutlich forschungsspezifischer und weiter fassten Nezel und Ghisla den Fokus bei ihrer gründlichen Vergleichsarbeit über die Teilbereiche und Schwerpunkte unterschiedlichster Projekte der deutschsprachigen Curriculumforschung. ${ }^{27}$ Unter dem Titel "Theorie und Verfahren zur Konstruktion und Analyse curricularer Wissensstrukturen» diskutieren sie die damals bestehenden acht Personengruppen und deren Projekte. ${ }^{28}$ Zwei davon waren im Schweizer Kontext angesiedelt: zunächst eine Gruppe um Heinz Moser, damals an der Universität Zürich mit einem Projekt zur politischen Bildung. Heinz Moser publizierte ferner in der «Zeitschrift für Pädagogik» zu «Curriculumforschung und Ideologie ${ }^{29}$ und doktorierte mit einer Arbeit zur «Handlungsorientierten Curriculumforschung». ${ }^{\circ}$ In seinen Publikationen übte er teilweise harsche Kritik an der Curriculumforschung als «Modenwissenschaft», welcher ein technologisches Unterrichtsverständnis zugrunde liege. Die emanzipatorische Komponente von Lernprozessen werde nicht berücksichtigt. ${ }^{31}$ Darüber hinaus problematisierte Moser zusammen mit Peter Roth und Roger Vaissière in einer Vorlesung am pädagogischen Institut 1973 weitere Aspekte der Curriculumund Unterrichtsforschung, etwa die unausgereifte Lernzielformulierung oder die Schwierigkeiten in der Auswahl von Unterrichtsinhalten. ${ }^{32}$

Ebenfalls im Kontext der Schweizer Forschung erwähnt wurde die Freiburger Arbeitsgruppe für Lehrplanforschung. Nezel und Ghisla stützten ihre Einordnungen auf ein ausgewähltes Projekt der FAL, wovon sie besonders die Planungs- und Implementationsorientierung heraushoben. Sie betonten die pro-

Aregger und Gretler, I983.

Nezel und Ghisla, I 977.

Präsentiert und diskutiert wurden auch die Arbeiten von Saul B. Robinsohn, Herwig Blankerz, Karl-Heinz Flechsig, Hartmut von Hentig, Dieter Lenzen und Rudolf Schörken.

H. Moser, I97I.

H. Moser, I974.

Ebd., S. 7.

Nezel und Ghisla, I977, S. 86. 
jektgebundenen Arbeiten zur Curriculumkonstruktion, welche sie schliesslich dem Vorwurf eines «theorielosen Pragmatismus» aussetzten. ${ }^{33}$

In Nezels und Ghislas Artikel nicht erwähnt, aber insbesondere für die Lehrplanentwicklung etlicher Berufsschullehrpläne verantwortlich war Rolf Dubs vom Institut für Wirtschaftspädagogik an der Universität St. Gallen.34 Er hatte sich ebenfalls zur Curriculumforschung geäussert, wenngleich weniger theoriegenerierend als entsprechende erziehungswissenschaftliche Bemühungen kommentierend. ${ }^{35}$ Darüber hinaus betreute Dubs die Dissertation von Hans Jörg Moser, welche nach den politischen Ursachen von Curriculumreformen fragte. Moser kam darin zum Schluss, dass nicht die gesellschaftlichen Problemlagen bestimmte Curriculumreformen anstossen würden, eher dienten sie im Nachhinein zur Legitimation der Reformen. Die verursachenden Faktoren von Curriculumreformen machte Hans Jörg Moser bildungssystemintern in den kleinen, überschaubaren Verwaltungen und gegeben durch persönliche Konstellationen in bildungspolitischen Instanzen aus. ${ }^{36}$

Die Darstellungen zum Forschungsstand der Curriculumforschung in der Schweiz zeigen ein sehr heterogenes und gleichsam überschaubares Bild der einschlägigen Forschung in den I970er-Jahren. An der Universität St. Gallen standen der Bezug zur Berufsbildung und die praktische Lehrplanarbeit im Vordergrund. An der Universität Freiburg wurden einzelne theoretische Aspekte in die konkrete Projektarbeit an Lehrplänen integriert. Die Forschung in Zürich scheute sich nicht davor, die Curriculumforschung insgesamt einer kritischen Betrachtung zu unterziehen. Vor allem die Einordnungsversuche der SKBF zur Forschung unter dem Prädikat «Curriculum» verdeutlichen anschaulich, wie diffus der Forschungsbegriff in jenem Bereich war: Curriculumforschung konnte sowohl an einer Universität als Grundlagenforschung betrieben werden als auch praxis- beziehungsweise anwendungsbezogen in Lehrplanrevisionsprojekten.

Das folgende Teilkapitel nimmt diesen Faden wieder auf, führt eine systematisierende Präzisierung zur gegenseitigen Abgrenzung von Grundlagenforschung und Anwendungsforschung ein und ordnet die Curriculumforschung in der Schweiz in die übrige Forschung im pädagogischen Bereich ein. Dazu werden die verschiedenen Umfragen zur Forschungsförderung der I970erJahre berücksichtigt, sie werden eingebettet in die Praxis der Forschungsförderung der Schweiz, und eine Analyse der Fördermittel des Nationalfonds wird präsentiert.

33 Ebd.

34 Dubs, Metzger und Hässler, 1973.

35 Dubs, 1974, 1976.

36 H. J. Moser, I980. 


\subsection{Förderung der Curriculumforschung in der Schweiz}

$\mathrm{Ab}$ den 1960er-Jahren war die Schweiz wie ihre Nachbarländer mit einer Entwicklung konfrontiert, die gegenwärtig als Bildungsexpansion bezeichnet und beschrieben wird. ${ }^{77}$ Der starke Anstieg der Geburtenzahlen in der Schweiz stellte die Kantone als Träger von Volksschule, Gymnasium, Lehrerinnen- und Lehrerbildung und teilweise von Hochschulen vor neue Herausforderungen. Die vollzogene Öffnung der höheren Bildung wie auch der Ausbau in allen Bereichen des Bildungswesens bis hin zu den Bildungsverwaltungen sollten mit den Mitteln verlässlicher Planung angegangen werden. ${ }^{38}$ Planungsstudien entwarfen Szenarien zur Hochschulentwicklung, etwa zum künftigen Bedarf an Lehrpersonen oder Schulbauten. Auch die Akteure der Wissenschaftspolitik in der Schweiz orteten Koordinations- und Entwicklungsbedarf, einerseits angesichts bildungsexpansionsbedingter Herausforderungen, andererseits aufgrund der Volksinitiative für Schulkoordination von I969. ${ }^{39} \mathrm{Im}$ Zuge dessen legte auch der Schweizerische Wissenschaftsrat ${ }^{40}$ Studien zur Situation der Forschung in der Schweiz vor. ${ }^{4}$

\subsubsection{Ermittlung der dringlichen Forschungsbedürfnisse der Schweiz}

Der Zweck solcher Studien war die Eruierung dringender Forschungsbedürfnisse in der Schweiz. Hierzu versendete am 26. Mai 1970 Kurt Schmid als Angehöriger des Schweizerischen Wissenschaftsrates einen Aufruf an die Leiter der schweizerischen Hochschulinstitute, Laboratorien und Seminare..$^{42}$ Mit diesem Schreiben war der Aufruf verbunden, in den kommenden zwei Monaten anhand zweier Fragebogen die Forschungsbedürfnisse und drängenden Problemstellungen in den einzelnen Subdisziplinen zurückzumelden. Nach mehrfacher Zwischenberichterstattung, schriftlichen Stellungnahmen dazu und einer Expertenkonferenz wurden die Resultate der Umfrage im Schlussbe-

37 Vgl. etwa Criblez, 2001a, 20 15; Geiss, 201 8; Kussau und Oertel, 200 I.

38 Zur «Errechenbarkeit» der Zukunft und zur einsetzenden Planungseuphorie vgl. Stettler, I 994.

39 Zum Wandel der Schweizer Forschungspolitik vgl. Gees, 2016; zur Bedeutung der Volksinitiative von 1969 für die Schulkoordination vgl. Criblez, 2007a; Manz, 20 I I.

40 I965 zunächst ohne gesetzliche Grundlage gegründet, etablierte sich der Schweizerische Wissenschaftsrat als beratendes Organ des Bundes für alle Fragen der Wissenschaftspolitik. Weiterführend Hafner, 2015 .

4I Criblez, 2007, S. 209.

42 K. Schmid, Dringende Forschungsbedürfnisse in der Schweiz, 26. 5. 1970, Archivalien der FAL. 
richt des Schweizerischen Wissenschaftsrates publiziert. ${ }^{43}$ Als förderungswürdige Bereiche genannt wurden Bildungsorganisation und Bildungsplanung, die Vorschulerziehung, Lehr-Lern-Prozesse in Mathematik und Sprachunterricht sowie der Bereich der Berufsbildung. Auch die Curriculumforschung wurde zu den dringend zu fördernden Bereichen der Bildungsforschung gezählt. Die Curriculumforschung müsse sich gemäss dem Bericht zukünftig auf die folgenden fünf Gebiete erstrecken: I. Analyse bestehender Lehrpläne, 2. Verfahren der Curriculumkonstruktion, 3. Ermittlung und Formulierung von Bildungszielen, 4. Erforschung von Unterrichtsmodellen, Unterrichtsmitteln und Kontrollverfahren und 5. Curriculumtheorie. ${ }^{44}$

Nicht nur die Nennung der Curriculumforschung unter den förderungswürdigen Forschungsbereichen ist hier herauszuheben, auch ihre Verortung im Feld der pädagogischen Forschung ist bemerkenswert: Der Schweizerische Wissenschaftsrat betrachtete Curriculumforschung wie die anderen zu fördernden Bereiche offenbar als Teile der Bildungsforschung und verwendete damit einen Begriff, den der Nationalfonds nur kurzzeitig verwendete, wie weiter unten noch genauer ausgeführt wird.

$\mathrm{Zu}$ den begrifflichen Nuancen rund um die Benennung der Teilgebiete der Pädagogik legte Armin Gretler in der «Revue française de pédagogie» 1979 einen Rückblick auf die pädagogische Forschung und Lehre von 1974 bis 1977 in der Schweiz vor. Dabei thematisierte er im Fazit ein Grundproblem der Disziplin. Sie hadere mit der eigenen Identität, was erkennbar sei an den Kontroversen zu ihrer Namensgebung. Auch wenn es gute Gründe gäbe, sich für das eine oder andere zu entscheiden, stünde doch die Frage im Raum: «[...] faut-il dire les sciences de l'éducation ou la science de l'éducation? En allemand [...] le problème est encore plus compliqué: Bildungsforschung, Bildungswissenschaft et Erziehungswissenschaft, les deux derniers chaque fois au singulier ou au pluriel, cinq termes donc au total, sont au choix pour désigner le domaine dont nous parlons.»45

Gretler entschied sich bei der Wahl eines Überbegriffs für «Bildungsforschung», weil dieser ihm am globalsten erschien. Der Schweizerische Nationalfonds wiederum folgte, wie unten weiter ausgeführt werden wird, einer anderen Systematik.

Neben den Studien des Schweizerischen Wissenschaftsrates wurden bis 1975 weitere Erhebungen mit Bezug auf die Schweizer Forschung im Bildungsbereich durchgeführt, diesmal von den Bildungsministerien des Europarates und mehrfach von der pädagogischen Kommission der EDK, welche eine Subkom- 
mission einberufen hatte zur Findung von Themen für die geplanten nationalen Forschungsprogramme. ${ }^{46}$ Über alle Planungsstudien hinweg betrachtet zeichnete sich keine klare Präferenz für einen der Forschungsbereiche ab. Samuel Roller ${ }^{47}$ vom Institut romand de recherches et de documentation pédagogiques (IRDP) führte die Unentschlossenheit auf eine fehlende Forschungspolitik in der Schweiz zurück. Ebenso wie Gretler war er der Ansicht, dass Unklarheit darüber herrsche, «was unter Bildungsforschung verstanden werden muss, wer voll zuständig ist jene zu betreiben, woraus ein Forschungszentrum besteht und schliesslich welche Zielsetzungen angestrebt werden sollen».48

Wohl wurde 1975 auf Initiative der SKBF die Schweizer Fachgesellschaft gegründet, für deren Bezeichnung man sich auf «Schweizerische Gesellschaft für Bildungsforschung (SGBF)» geeinigt hatte. In der Frage um die zu fördernden Bereiche der Forschung hatte sich aber keines der bisherigen Themen durchsetzen können. 1976 wurden für das erste nationale Forschungsprogramm im Bildungsbereich 5,9 Millionen gesprochen, ${ }^{49}$ und zwar im Themenbereich «Probleme der sozialen Integration in der Schweiz» unter der Leitung von Heinrich Tuggener. ${ }^{50}$ Die Curriculumforschung war in diesem Förderungsformat leer ausgegangen. Doch haben Curriculumforscher beim SNF mehrfach erfolgreich Forschungsgelder einwerben können. Im Folgenden soll daher die Vergabe von Fördergeldern im Bereich der Schweizer Forschung im Bildungsbereich in den I970er-Jahren analysiert werden. So wird auch die Möglichkeit gegeben, den Stellenwert der Curriculumforschung anhand der Fördermittelvergabe zu diskutieren.

46 Roller, 1975, S. 2 f.

47 Samuel Roller (1912-2003) erwarb 1930 das Lehrerpatent im Kanton Neuenburg, um anschliessend einige Jahre in den Schuldienst zu treten. Nach einem weiterführenden Studium der Erziehungswissenschaft unterrichtete er bis zu seiner Promotion 1955 in Pädagogik Lehramtsstudenten am Lehrerseminar in Genf. Zwischenzeitlich leitete er bis I965 die Abteilung für pädagogische Forschung des Genfer Erziehungsdepartementes und teilte sich mit Jean Piaget das Direktorat des Genfer Institut des sciences de l'éducation. 1970-1977 war er Direktor des neu gegründeten Institut romand de recherches et de documentation pédagogiques (IRDP) in Neuenburg.

48 Schwarzmann, 1985 , S. I 3 .

49 Ohne Autor, 1976.

50 1972-1989 lehrte und forschte Heinrich Tuggener an der Universität Zürich im Bereich der Sozialpädagogik und er leitete etliche Forschungsprojekte. Die ausserordentliche Professur für Pädagogik und Sozialpädagogik wurde 1976 in eine ordentliche Professur übergeführt. Zur selben Zeit bekleidete Tuggener das Amt eines Forschungsrates beim SNF. 


\subsubsection{Förderung der Curriculumforschung durch den Schweizerischen Nationalfonds}

Der Schweizerische Nationalfonds zur Förderung der wissenschaftlichen Forschung ${ }^{\text {II }}$ (SNF) wurde 1952 gegründet. Mit der Verwendung des Begriffs «wissenschaftlich» im Namen und in der Stiftungsurkunde wurde eine genauere Bestimmung dessen, was förderungswürdig war, vorgenommen, der getreu der SNF in den Folgejahren Hunderte Projekte förderte. Nicht die Forschung mit Verwendungszweck oder kommerziellen Absichten, sondern «zweckfreie, rein theoretische Forschung, die Forschung also, die nicht an der praktischen Anwendbarkeit orientiert ist», ${ }^{52}$ wurde vom SNF finanziell unterstützt. ${ }^{53}$ Im weiteren Kapitelverlauf gilt diese Definition in Bezug auf die Analyse und Diskussion der in der Schweiz geleisteten Forschungsarbeiten im Curriculumbereich. Projekte mit Bezügen zur Curriculumforschung, welche diesem Anspruch nicht gerecht wurden, sind Gegenstand der Kapitel drei und vier.

Der SNF publiziert jährlich einen Bericht und legt darin Rechenschaft über seine Tätigkeiten ab. Für den hier zu untersuchenden Zeitraum von I970 bis 1980 wurden in den Berichten die jeweils ausgebrachten Gelder zur Forschungsförderung ausgewiesen und in drei Abteilungen gegliedert: Geisteswissenschaften (I), exakte und Naturwissenschaften (II), Biologie und Medizin (III). Die Gelder der geisteswissenschaftlichen Abteilung wurden - exemplarisch der Bericht für 1970 - jeweils separat für die drei folgenden Förderbereiche angegeben: I. Philosophie (50000 Franken), 2. Psychologie, Pädagogik, Anthropologie (927 000 Franken) und 3. Soziologie und politische Wissenschaften (763000 Franken). Anhand der Tabelle I wird ersichtlich, dass die Benennungen der einzelnen Bereiche in der Abteilung I während eines Jahrzehnts an einigen Stellen verändert wurden.

Die politischen Wissenschaften wurden 1972 in «Politikwissenschaften» umbenannt, bis sie 1977 von der Soziologie abgespalten und ihre Fördergelder

5I Der SNF wurde in der Absicht gegründet, die immer teurer werdende Grundlagenforschung in der Schweiz voranzubringen. Zwar haben einzelne Forschungsbereiche seit dem Kriegsende im Jahr 1945 durchaus Fördergelder des Bundes erhalten, die Projekte mussten aber immer auf Arbeitsbeschaffung ausgerichtet sein, was die Geisteswissenschaften und die jungen Nachwuchsforschenden systematisch benachteiligte. Weiterführend Schwarzmann, I985.

52 Ebd., S. I3.

53 Seit den Gründerjahren hat sich die Vergabepraxis beim SNF teilweise gewandelt. Schon früh berücksichtigte der SNF den Praxisbereich in der Forschungsförderung, auch wenn dabei arbeitsmarktpolitische Argumente im Vordergrund standen. Weiterführend dazu Criblez, 1995. Derzeit fördert der SNF auch «anwendungsorientierte Grundlagenforschung». Vgl. dazu www.snf.ch/de/derSnf/forschungspolitische_positionen/anwendungsorientierte_grundlagenforschung/Seiten/default.aspx, abgerufen am 9. I0. 2018. 
Tab. 1: Fördergelder des Nationalfonds für Abteilung I: Geisteswissenschaften, 1970-1980 (in 1000 Franken)

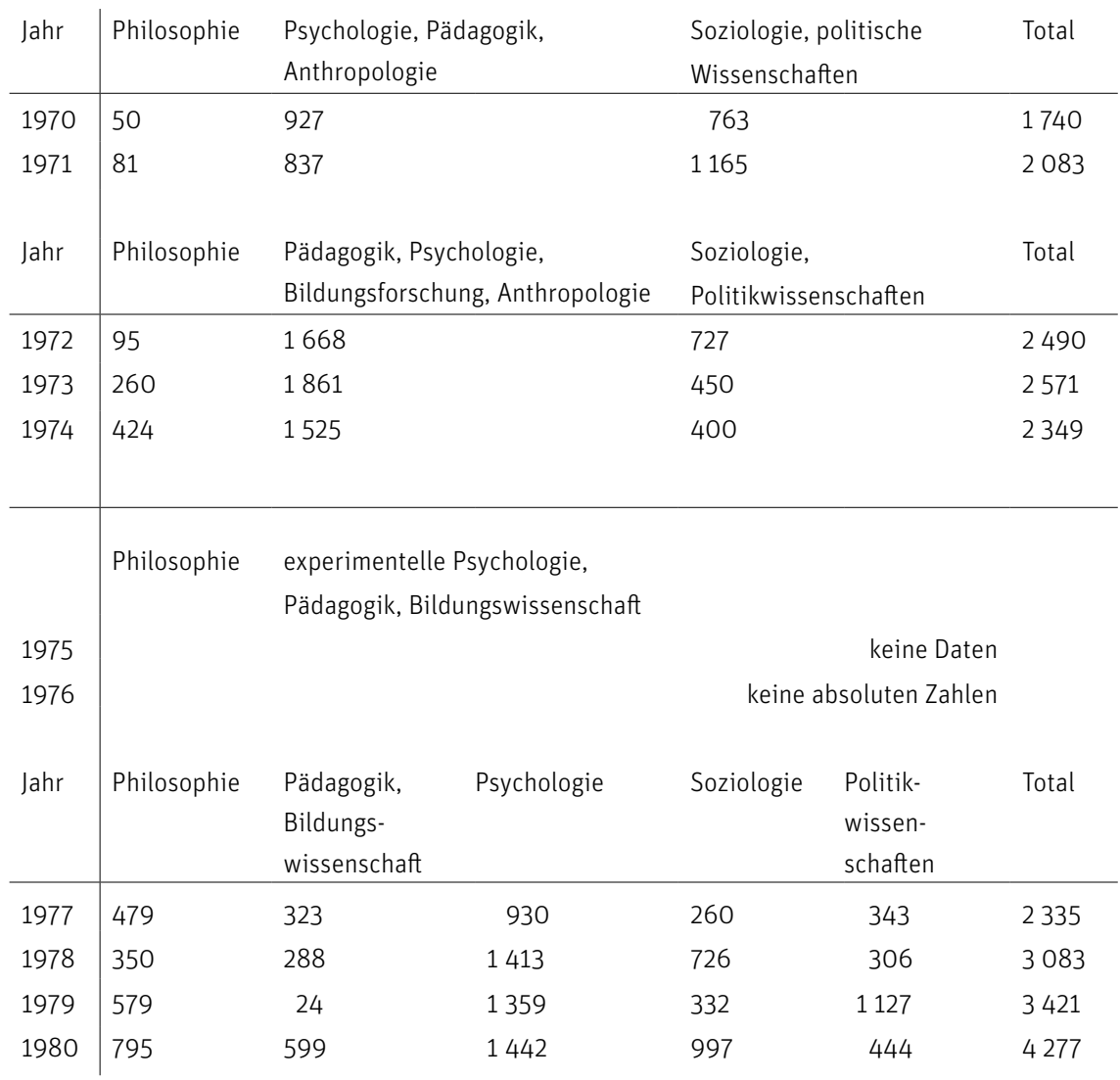

Quelle: Jahresberichte SNF

separat ausgewiesen wurden. Der Pädagogik zugeordnet wurde 1972 die neue Kategorie «Bildungsforschung», drei Jahre später wurde sie durch «Bildungswissenschaft» ersetzt, die Anthropologie verschwand aus jener Kategorie und die Psychologie wurde nach einem kurzen Intermezzo als «experimentelle Psychologie» letztlich separiert.

Zur Einführung der Kategorie «Bildungsforschung» schrieb der SNF: «Allgemein zeichnet sich eine deutliche Tendenz zur Wahl von Projekten ab, die im en- 
geren oder weiteren Sinn zur ‘Bildungsforschung〉 gezählt werden dürfen.» ${ }^{4} 4 \mathrm{Die}$ Ursache für jene neue Kategorie wird 1973 darin gesehen, dass «in neuerer Zeit [...] die empirisch orientierten Forschungsrichtungen der Pädagogik (vor allem der Didaktik), der Psychologie, der Soziologie, der Bildungsökonomie und der Medizin hinzu[kamen]. Dies führte zu einer Annäherung, gegenseitigen Ergänzung und Befruchtung geisteswissenschaftlich-spekulativer und empirisch-experimenteller Forschungsmethoden, Betrachtungsweisen und Standpunkte.»ss

Die Curriculumforschung - es sei daran erinnert, dass die Forschenden beabsichtigten, die schulischen Lehrpläne zum Zweck der rationalen Schulreform auf wissenschaftlich-rationaler Grundlage neu zu schreiben - wurde weiterhin der Pädagogik und damit im Jargon des SNF der «spekulativ-hermeneutisch» ${ }^{6}$ ausgerichteten Forschungsrichtung zugewiesen.

Tabelle I zeigt ferner die Entwicklung der vom SNF zur Verfügung gestellten Forschungsgelder. Am Beispiel der Philosophie, deren Fördergelder in den Jahresberichten des SNF immer explizit ausgewiesen wurden, kann anschaulich ein mehr oder minder kontinuierlicher Zuwachs an gesprochenen Forschungskrediten beobachtet werden. Ebenso wie für die Soziologie und die Politikwissenschaften bis 1974 die Fördergelder nur addiert ausgewiesen wurden, kann auch für Psychologie und Pädagogik bis 1977 kein eigenständiger Betrag an Fördergeldern abgelesen werden. Um Genaueres zur Curriculumforschung im Kontext der Forschungsförderung des SNF sagen zu können, wird im Folgenden der Fokus auf die einzelnen Forschungsprojekte gerichtet.

Von 1968 bis 1979 hat der SNF Gelder gesprochen für knapp dreihundert Projektverlängerungen und Projekte, welche im weitesten Sinne einen Bezug zu Fragen der Bildung und Erziehung aufweisen. Für die Grafik 2 sind die Projekte entsprechend der beantragenden Institution ${ }^{57}$ in die folgenden Kategorien eingeteilt worden: r. Psychologie ohne Curriculumforschung, 2. Pädagogik

54 Jahresbericht SNF 1972, S. 4I, StAZH, DSP 49.

55 Ebd.

56 Jahresbericht SNF 1973, S. 42, StAZH, DSP 49.

57 Die Zuweisung der Projekte in Abhängigkeit von einer beantragenden Institution hiess zum Beispiel: Prof. Ulrich Moser vom psychologischen Institut der Universität Zürich hat I97 I beim SNF Gelder für drei Jahre eingeworben. Demzufolge wird das Projekt der Kategorie «Psychologie» zugewiesen. Mit dieser Kategorisierungspraxis werden Aussagen zu institutionellen Verhältnissen möglich. Auf die Zuordnung in Abhängigkeit von einzelnen Forschungsprojekten und ihrer Beschreibung wird verzichtet. In Einzelfällen, gerade im Forschungsbereich der pädagogischen Psychologie, wären Zuweisungsprobleme häufiger gewesen, zumal solche Projekte oftmals der Pädagogik oder der Psychologie zugeteilt werden konnten. 
Grafik 2: SNF-Fördergelder Geisteswissenschaften I für Bildung und Erziehung

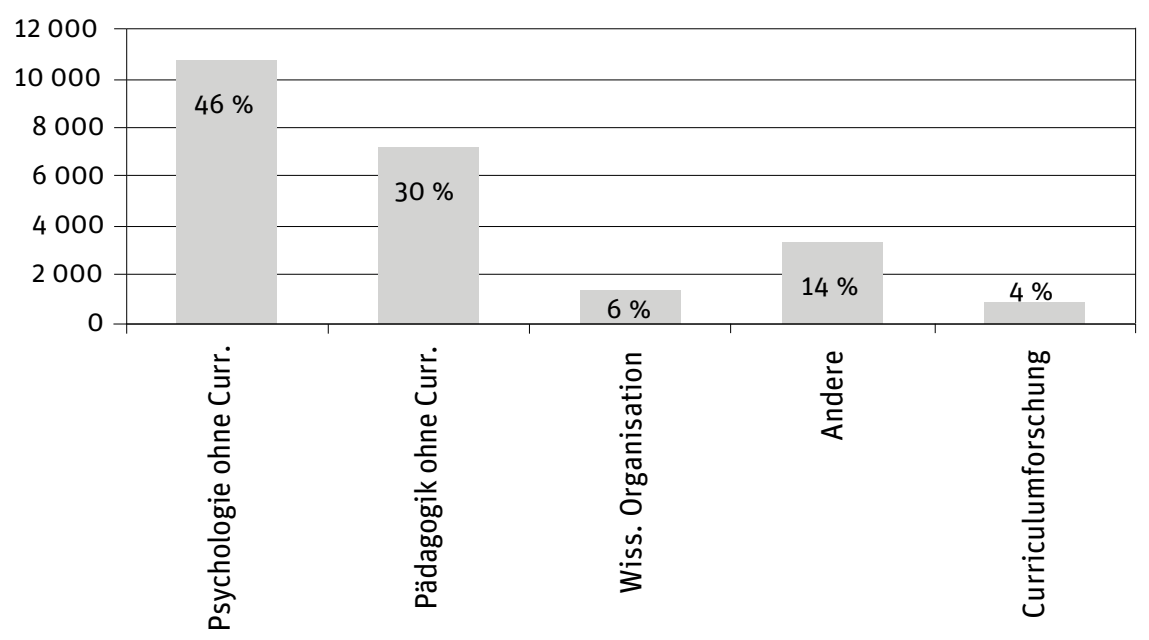

Quelle: Projektdatenbank des SNF (eigene Darstellung)

ohne Curriculumforschung, 3. wissenschaftliche Organisation, ${ }^{8} 4$ 4. andere,, 9 5. Curriculumforschung. ${ }^{60}$

Vergleicht man die Verteilung der Fördergelder zwischen Psychologie und Pädagogik über den ganzen Zeitraum, so ist festzuhalten, dass die psychologische Forschung zahlenmässig immer mehr Projekte bekleidet hat, und diese Projekte waren in der Regel besser finanziert. Dies erklärt den hohen Anteil der psychologischen Forschung am gesamten Forschungsetat für Bildung und Erziehung.

Der Anteil der Curriculumforschung an den insgesamt für Bildung und Erziehung gesprochenen Mitteln des SNF lag bei 4 Prozent. Insgesamt handelte

58 «[W]issenschaftliche Organisation» meint: Koordinationsgelder für ein nationales Forschungsprogramm oder Gelder für eine Informationsveranstaltung.

59 Die Kategorie «andere» umfasst Projekte, welche vom SNF zwar zu Bildung und Erziehung gezählt werden, wo die beantragende Institution aber nur entfernt Bezüge zu Pädagogik und Psychologie aufweist, etwa Projekte des Departementes für Architektur in Lausanne, der Berufsberatungsstelle in Biel oder der Sozialforschungsstelle an der Universität Zürich.

60 Die Projekte der Kategorien Soziologie und Politikwissenschaft sind zwar Teil dieser Analyse, sofern sie gemäss dem Nationalfonds einen Bezug zu Bildung und Erziehung haben, werden aber in der Grafik nicht abgebildet. 
es sich um 875000 Franken, verteilt auf drei Projekte, wovon zwei mehrmals verlängert worden waren. Das erste Projekt unter dem Titel «Strukturanalyse der schweizerischen Lehrerbildung» mit einer Laufzeit von einem Jahr ab 1968 wurde verhältnismässig bescheiden finanziert mit 6000 Franken. Ein zweites Projekt zu «Methoden der Curriculumkonstruktion» dauerte von 1970 bis I 975 und war mit knapp 600000 Franken alimentiert. Drittens wurde ein Projekt gefördert mit dem Titel «Struktur und Funktion didaktischer Bezugssysteme für die Unterrichtsplanung», welches dann unter dem Titel «Leitideen in der Volksschule Freiburg» verlängert wurde und mit insgesamt 277000 Franken gefördert wurde. Im Übrigen zählen die einzelnen Projekttranchen der Curriculumforschung mit Ausnahme der letzten Projektverlängerung und der allerersten Anschubhilfe zu den zwanzig finanzstärksten im betrachteten Zeitraum.

Bemerkenswerterweise sind alle vom Nationalfonds geförderten Projekte der Curriculumforschung an derselben Universität von derselben Gruppe von Forschenden eingeworben worden: der Freiburger Arbeitsgruppe für Lehrplanforschung (FAL). Die Analyse der Projekte des SNF weist zudem deutlich darauf hin, dass sich in der Schweiz nur diese eine Forschungsgruppe mit Curricula insofern wissenschaftlich auseinandergesetzt hat, als ihre Arbeit vom SNF gefördert wurde.

\subsection{Die Freiburger Arbeitsgruppe für Lehrplanforschung (FAL)}

«[D]ie Freiburger Arbeitsgruppe für Lehrplanforschung (FAL) [ist] das einzige schweizerische Team, das sich ständig mit dem Curriculum als Forschungsgebiet befasst. Es besteht zur Zeit die Aussicht, dass sich aufgrund des rasch wachsenden Problembewusstseins der curricularen Frage bald weitere Forschungsgruppen bilden. $»^{61}$

Karl Frey als Gründer und erster Leiter der FAL verfasste diese Einschätzung für einen 1970 publizierten Zeitschriftenartikel zu einem Zeitpunkt, als die Gründung der FAL bereits zwei Jahre zurücklag und sie personell im Ausbau begriffen war. Damals hatten die Mitarbeiter ${ }^{62}$ der FAL bereits ihr drittes Anwendungs- und Entwicklungsprojekt einwerben können. Unter dem Titel «Entwicklung und begleitende Analysen eines Curriculums der Weiter-

6 I K. Frey, 1970, S. 70.

62 Wenn hier wie im Folgenden von «Mitarbeitern» der FAL die Rede ist, handelt es sich keinesfalls um eine Nichtbeachtung geschlechtergerechter Sprache. Tatsächlich waren in der FAL alle an Forschung und Entwicklung beteiligten Personen Männer. 
bildungsschule Zug» (EBAC-WS) planten und entwickelten sie ab I970 einen zweijährigen Diplomschulgang, welcher im Anschluss an die obligatorische Schulzeit absolviert werden konnte. ${ }^{63}$ Daneben entwarf die FAL einen Entwicklungsplan für die Schulen im Kanton Schwyz und ein Curriculum für die Primarschulen Deutschfreiburgs (EBAC-PS). Im Oktober 1972 konnte die FAL die ersten nennenswerten Projektförderungsgelder beim SNF zum Thema «Methoden der Curriculumkonstruktion» einwerben.

Die Reihung der angeworbenen Projekte lässt vermuten, dass die FAL selbst zunächst ein eigenes Problembewusstsein im Curriculumbereich anlässlich von konkreten Entwicklungsprojekten entwerfen musste, bevor sie Fördergelder des Nationalfonds beantragen konnte. Insofern werden die folgenden Analysen die Gründung der FAL an der Universität Freiburg einordnen, bevor die Forschungsleistungen der FAL wissenschaftsgeschichtlich hinsichtlich der Ausarbeitung und Weiterentwicklung von Ideen und Konzepten wie auch in Bezug auf den Output in Form von Dissertationen und Artikeln in Fachzeitschriften untersucht werden. Dabei wird deutlich, inwieweit die Mitglieder der FAL, gegeben durch intensive Zusammenarbeit in der Gruppe, einen gemeinsamen Denkstil ${ }^{64}$ ausgeprägt haben. Die Tätigkeiten der FAL abseits der wissenschaftlich-universitären Zusammenhänge stehen später in dieser Arbeit im Zentrum der Analyse.

\subsubsection{Die Gründung der FAL}

Bevor Karl Frey seine Studienzeit 1966/67 an der Universität Freiburg (Schweiz) abschloss, hatte er bereits zwei Semester im belgischen Löwen am Institut supérieur de la philosophie studiert und anschliessend von 1963 bis I 966 an der Universität Münster Psychologie, Pädagogik und Theologie belegt. Dann arbeitete er zunächst an einer Dissertation mit theologischer Ausrichtung. ${ }^{65}$ Auf Anraten von Norbert Luyten ${ }^{66}$ hat Frey sein fertiges Manuskript

63 Das EBAC-PS-Projekt fällt in die Zeit der Gründungsphase der Diplommittelschulen. Zur Geschichte dieses Schultyps weiterführend Leemann und Christian, 20 I I.

64 Fleck, I935.

65 In den privaten Archivalien von Karl Frey ist ein entsprechendes Manuskript zu finden mit dem Titel «Die Inputation sittlichen Handelns und des Gewissens».

66 Norbert A. Luyten war geweihter Priester sowie studierter Theologe und Philosoph. 1948-1979 war Luyten ordentlicher Professor der Naturphilosophie und philosophischen Anthropologie an der Universität Freiburg. Er gilt laut dem «Historischen Lexikon der Schweiz» als Förderer der interdisziplinären Forschung und interessierte sich für den psychologischen und pädagogischen Unterricht. Luyten war als Präsident der Institutskommission in die Arbeiten der FAL strategisch eng eingebunden. 
aber nie abgegeben. ${ }^{67}$ Stattdessen nahm er ein neues Thema aus seiner Lehrtätigkeit am deutschsprachigen Lehrstuhl für Pädagogik von Ludwig Räber auf. Mit einigen Lehramtsstudierenden führte Frey ein Seminar zu Schweizer Lehrplänen der Real-, Sekundar- und Bezirksschulen durch. Daraus resultierte eine zweibändige Promotionsarbeit, ${ }^{68}$ welche im ersten Teil einen erstmaligen Überblick über die aktuellen kantonalen Lehrpläne und ihre Vielfalt bot. Diesen ersten Teil hat Frey ein Jahr darauf auch in der Zeitschrift der Schweizerischen Konferenz der kantonalen Erziehungsdirektoren veröffentlicht. ${ }^{69}$ Der zweite, darauf aufbauende Band forderte auf zur Koordination und Neugestaltung jener Lehrpläne und stellte darüber hinaus eine Bildungskonzeption zur Diskussion, die zur Neuerarbeitung der Lehrpläne aufrief, zumal die alten als Stichwortsammlungen von Stoffinhalten konzipiert seien. ${ }^{70}$

Dieser Arbeit folgten noch einige weitere, die ebenfalls darauf angelegt waren, über den aktuellen Stand der Lehrpläne im Bildungswesen in der Schweiz einen Überblick zu bieten. Die Lage war unübersichtlich, zumal selbst für die oben erwähnte Untersuchung von 1968 sechs Kantone ihre Lehrpläne nicht vollständig zur Verfügung stellen konnten. ${ }^{71}$ Curriculumforschung hiess zunächst, eine Analyse der bestehenden Lehrpläne vorzunehmen. Dieses Vorgehen «dient dem Aufbau der künftigen Curricula», wie Frey in einem Artikel des «International Review of Education» verlauten liess und weiter ergänzte, es entspreche einem bildungspolitischen Bedürfnis. ${ }^{72}$

Der Gegenstandsbereich der Lehrpläne oder, in der Sprache der Zeit, der «Curricula» war damit hinreichend problematisiert und legitimierte weitere Anstrengungen in diese Richtung. Unter der Leitung von Frey entstanden am Lehrstuhl von Ludwig Räber weitere Untersuchungen zur Ausbildung der Lehrpersonen der Volksschule ${ }^{73}$ sowie zur Ausbildung der Lehrpersonen an den Gymnasien.74 Auch der Vergleich von Aufbau und Inhalten der Lehrerweiterbildung in der Schweiz wurde Gegenstand von wissenschaftlichen Analysen.75

Für diese Arbeiten benötigte Karl Frey zusätzliches Personal. Er begann geeignete Studienabgänger aus seinen Seminaren anzusprechen und offerierte ihnen

67 A. Frey, 2017.

68 K. Frey, 1968b, I968a.

69 K. Frey, i969c.

70 Vernetz, I968.

7I Das Problem wiederholte sich acht Jahre später. 1976 trugen Mitarbeiter der FAL die damals gültigen Lehrpläne der Primarschule aller Kantone im Auftrag der EDK zusammen und mussten wiederum auf etliches Material verzichten, weil es ihnen nicht zur Verfügung gestellt werden konnte.

72 K. Frey, I969d, S. 24.

73 K. Frey, 1969b.

74 Horn, I969.

75 Kaiser, 1970. 
eine Mitarbeit in seinen Projekten. ${ }^{6}$ Bald verfügte er über ein Team von acht Mitarbeitern, welche unter seiner Leitung engagiert waren und daneben an ihren Qualifikationsarbeiten schrieben. Ein Gründungsdatum der Freiburger Arbeitsgruppe ist keines bekannt, auch nicht ein Datum für ihre Auflösung. Anhand des Arbeitsbeginns für die neu eingestellten Mitarbeiter kann immerhin näherungsweise bestimmt werden, wann die FAL ihre Arbeit aufgenommen hat.

$\mathrm{Zu}$ den ersten Mitarbeitern der FAL mit Beginn I968/69 zählten Urs Peter Lattmann, Iwan Rickenbacher und Ralf Horn. Bis 1970 kamen Joseph Eigenmann, Kurt Bossart und Anton Strittmatter dazu. Ab i97 I zählten Kurt Aregger, Urs Isenegger, Bruno Santini, Joe Brunner und Edi Schellhammer mit zur FAL. In demselben Jahr haben die ersten Mitglieder die FAL auch schon wieder verlassen, darunter der einstige Gründer Karl Frey. Er war einem Ruf ans Leibniz-Institut für die Pädagogik der Naturwissenschaften und Mathematik (IPN) in Kiel gefolgt. Zwischen 1972 und 1974 hat die FAL fünf neue Mitarbeiter anstellen können: Ueli Heiniger, Xaver Winiger, Albin Niedermann, Beat Mayer und Anselm Rohner. Gleichzeitig haben Aregger, Rickenbacher, Bossart und Schellhammer die FAL verlassen. Ab 1975 sind noch drei Mitarbeiter neu bei der FAL angestellt worden: Daniel Huber, Ernst Preisig und Kurt Schmid. Mit Blick auf die Dauer des Engagements in der FAL ist erstens festzuhalten, dass einige Mitglieder die Arbeitsgruppe nach zwei oder drei Jahren wieder verlassen haben, was eine beachtliche Fluktuation der Mitarbeiter bedeutete. Hingegen sind von den insgesamt 20 Forschern acht während fünf bis acht Jahren in der FAL aktiv gewesen. Dazu zählen Brunner, Eigenmann, Santini, Heiniger, Rickenbacher, Mayer, Winiger und Strittmatter. Zweitens ist es in Bezug auf das Eingangszitat von Karl Frey wichtig festzuhalten, dass die FAL nicht nur die einzige Forschergruppe in der Schweiz war, die sich vorwiegend der Curriculumforschung annahm, sondern auch eine vergleichsweise grosse Gruppe, die bis zu neun ständige Mitarbeiter zu einem Themenbereich zusammenführte. ${ }^{77}$

\subsubsection{Institutionelle Verortung der FAL an der Universität Freiburg}

Wie lässt sich die Freiburger Arbeitsgruppe institutionell am pädagogischen Institut der Universität Freiburg verorten, zumal es sich nicht um eine kleine, informelle Gruppe an einem Lehrstuhl gehandelt hat, sondern um eine Gruppe, die mit ihrer Arbeitstätigkeit bald eigene Gelder einwarb und über die Univer-

76 Isenegger, 20I6; Lattmann, 20I 5; Rickenbacher, 2016.

77 Dazu exemplarisch der Tätigkeitsbericht der FAL 1974. Eigenmann und Strittmatter, 1975, Archivalien der FAL. 
sität hinaus Bekanntheit erlangte? Und inwiefern war die zugehörige Professur von Ludwig Räber in die Arbeiten eingebunden?

Im «Reglement der Freiburger Arbeitsgruppe für Lehrplanforschung» ${ }^{8}$ ist im ersten Artikel festgehalten, dass es sich bei der FAL um eine Zweckorganisation im pädagogischen Institut zur Durchführung pädagogischer Forschungsprojekte handelte. «Unter Forschungsprojekten sind zu verstehen die wissenschaftlichen Studien und Untersuchungen mit dem Ziel des Erkenntnisfortschrittes und der Dissemination von Forschungserkenntnissen.»79 Entstanden ist dieses Reglement erst nach zwei Jahren des Bestehens der FAL und nach einigen Unstimmigkeiten zwischen Mitgliedern der FAL und der Universität Freiburg. Mit Verwunderung und teilweise mit Argwohn sind an der Universität Freiburg die umtriebigen und teilweise lukrativen Geschäftigkeiten zu Lehrplan- und Schulreformbelangen dieser jungen Arbeitsgruppe zur Kenntnis genommen worden, die abseits von den eigentlichen Forschungsschwerpunkten am pädagogischen Institut und der Ausrichtung des Lehrstuhls von Ludwig Räber positioniert waren. ${ }^{8} \mathrm{Zu}$ Räbers Rolle für die FAL muss an dieser Stelle ein kleiner Rückblick auf seine Berufung an die Universität Freiburg eingeschoben werden.

Als Ludwig Räber als Rektor der Stiftsschule Einsiedeln am 9. Dezember I965 an die Universität Freiburg reiste, um seinen Vortrag im Rahmen der neu zu besetzenden Professur des deutschsprachigen Lehrstuhls für Pädagogik zu halten, war noch nicht abzusehen, dass er 1966 zwar berufen werden würde, aber nach acht Jahren wieder nach Einsiedeln zurückkehren würde. Bereits seine Berufung war umstritten, zumal die philosophische Fakultät seinen Namen nicht mit auf die Vorschlagsliste gesetzt hatte und seine Wahl damit erschwerte. ${ }^{81}$ Auch die diversen vertraulichen Anfragen bei anderen Professoren um die Einschätzung der fachlichen Eignung der Kandidaten brachten nur mehrdeutige Resultate hervor. ${ }^{{ }_{2}}$ Schliesslich gereichte dies Räber zum Vorteil, der 1966 seine Tätigkeit an der Universität Freiburg aufnahm und als Erstes Karl Frey als Assistenten anstellte.

Interessanterweise hat sich die FAL bei der Aushandlung ihres Reglements I97 I von Räber so weit wie möglich losgesagt. Bei den Regelungen zur Bestimmung

78 Reglement der FAL, 22. I. 197I, Pädagogisches Institut der Universität Freiburg, Archivalien der FAL.

79 Ebd.

80 Isenegger, 20I6; Lattmann, 20I5; Rickenbacher, 2016.

8 I Schnidinger, 17. 2. 1966, Universität Freiburg, Archivalien Dekanat der Philosophischen Fakultät.

82 Die vertraulichen Anfragen wurden sowohl vom Dekan, dem Präsidenten der Institutskommission wie auch von Eduard Montalta, dem Inhaber der Professur, verfasst und verschickt. 
des FAL-Rates wäre zu erwarten gewesen, dass hierzu der Lehrstuhlinhaber für Pädagogik der deutschsprachigen Abteilung bestimmt würde. Stattdessen wurde hierfür der Präsident der Institutskommission bestimmt. So hat sich die FAL institutionell als Zweckorganisation neben den beiden Lehrstühlen für Pädagogik in die philosophische Fakultät eingeordnet und konnte mit Norbert Luyten, dem Präsidenten der Institutskommission, einen Fürsprecher einbinden. Wenn über die Annahme von Forschungsarbeiten, die Wahl neuer Mitarbeiter oder den Einbezug von weiteren Personen zu entscheiden war, fiel der Einschätzung von Luyten reglementsgemäss grosses Gewicht zu. ${ }^{83}$

\subsubsection{Der Forschungsgegenstand der FAL}

Indem die FAL mit ihren ersten, oben beschriebenen Projekten einen Überblick über das Lehrplan- und Lehrerbildungswesen in der Schweiz erarbeitete und Entwicklungstendenzen aufzeigte, erschloss sie sich ein neu zu beforschendes thematisches Feld. Als wäre, mit Kuhn gesprochen, ${ }^{{ }^{4}}$ der Beginn einer Phase der Normalwissenschaft angebrochen, taten sich neue Fragehorizonte auf, neue oder wiederentdeckte Problemstellungen wurden aufgeworfen. Nun steht im Fokus, welche Fragen die Mitglieder der FAL besonders beschäftigte und auf welche Theorien und Konzepte sie sich bei ihren Überlegungen abstützten. Als Ausgangspunkt hierfür dienen die von den Mitarbeitern der FAL verfassten Promotionsarbeiten. Sie werden gruppiert nach den Forschungsschwerpunkten Lernziele und Curriculumforschung, Schulreform und Curriculumforschung sowie Lehrberuf und Curriculumforschung.

\section{Theorien des Curriculums}

Die Habilitationsschrift von Karl Frey zu «Theorien des Curriculums» ${ }^{85}$ war noch nicht druckreif, als Frey bereits von Karl Hecht, Professor am Institut für Pädagogik der Naturwissenschaften in Kiel (IPN), um Bewerbungsunterlagen angefragt wurde für die neu geschaffene Professur für Pädagogik. ${ }^{86}$ Am Institut in Kiel bestand grosses Interesse an Freys Forschung, infolge dessen er für den 4. September 1970 einer Einladung für ein Referat vor dem IPN-Kuratorium gefolgt war. ${ }^{87}$ Mit seinen Ausführungen scheint er die Zuhörenden überzeugt

83 Reglement der FAL, 22. I. I97 I, Pädagogisches Institut der Universität Freiburg, Archivalien der FAL.

84 Kuhn, I967.

85 K. Frey, 197I.

86 Karl Hecht an Karl Frey, I4 4 4. 1970, Archivalien von Karl Frey.

87 Karl Hecht an Karl Frey, 16. 7. 1970, Archivalien von Karl Frey. 
zu haben. Im April 197I und nach einigem Schriftenwechsel in Bezug auf das beste Vorgehen aufgrund der noch ausstehenden Habilitation an der Universität Konstanz erhielt Frey einen Ruf nach Kiel. ${ }^{88}$

Über seine Habilitationsschrift war wenig später in der «Zeitschrift für Pädagogik» zu lesen: «Schon das Thema dürfte eine große Zahl von Interessenten ansprechen, steht es doch gegenwärtig im Mittelpunkt der erziehungswissenschaftlichen Diskussion.» ${ }^{89}$ Frey ging darin der Grundfrage nach, welche wissenschaftsmethodischen Prinzipien und Ansätze den verschiedenen Theorien des Curriculums zugrunde liegen und wie sie sich zur curricularen Praxis verhalten. Dazu diskutierte und kategorisierte er bestehende Curriculumtheorien, weshalb er nicht umhin kam, eine eigene Definition des Curriculumbegriffs festzulegen, die da lautete: «Das Curriculum ist die systematische Darstellung des beabsichtigten Unterrichts über einen bestimmten Zeitraum als konsistentes System mit mehreren Bereichen zum Zwecke der optimalen Vorbereitung, Verwirklichung und Evaluation von Unterricht.»90

Mit dieser Definition legte Frey den Grundstein für die weitere Curriculumforschung in der FAL. Er schuf damit den Anknüpfungspunkt zu jenen weiteren Fragen, welcher sich die Mitarbeiter der FAL in ihren Dissertationen annahmen. Allein aus dieser Definition lassen sich Fragen ableiten wie: Wie feingliedrig sollte Unterricht geplant werden? Auf fünf Minuten getaktet oder als Grobplanung konzipiert für das kommende Halbjahr? Welche übergeordneten Bildungsziele sollten wie bestimmt werden? Ist dazu die Erhebung und Auswahl von Lernzielen notwendig? In welcher Sequenz sollten Lernziele arrangiert werden? Wie sollten Lernkontrollen konstruiert werden? Kurz: Wie sollte ein Curriculum konstruiert werden und wie das Curriculum die Instruktion in den Klassenzimmern steuern?

\section{Lernziele und Curriculumforschung}

Als Ausgangspunkt für seine Dissertation wählte Ralf Horn den Befund einer Studie von Karl Frey und Urs Peter Lattmann, wonach Lehrpersonen nach einer Schulung im Formulieren von Lernzielen in ihrem späteren Unterricht andere Lernziele setzen würden als zuvor, und zwar tendenziell solche, die einfacher zu entwickeln seien. ${ }^{91}$ Horn konzentrierte sich in seiner Studie infolgedessen auf die Beschreibung von Verhaltenszielen, welche «höhere kognitive

88 Im März 197 I lehnte Frey einen Ruf auf eine ordentliche Professur für Schulpädagogik in Berlin ab.

89 Treml, 1971, S. 307.

90 K. Frey, 197I, S. 50.

9I K. Frey und Lattmann, 1970. 
Funktionen» voraussetzten..$^{92}$ Zur Beschreibung jener Verhaltensziele wertete Horn Leistungen von Schülerinnen und Schülern bei Prüfungsaufgaben aus dem naturwissenschaftlichen Unterricht aus und verortete sie im Referenzrahmen der bloomschen ${ }^{93}$ Klassifikationen.

Ebenfalls mit Lernzielen setzte sich Urs Isenegger auseinander. Sein Forschungsvorhaben entstand aus der Zusammenarbeit zwischen der FAL und dem pädagogischen Institut der Universität Zürich im Rahmen des BIVOProjektes. ${ }^{94}$ In seiner Dissertation analysierte er zunächst empirische Verfahren zur Lernzielerhebung unter curricularen Aspekten. ${ }^{5}$ Hierbei bezog er sich auf bereits bestehende Überlegungen von Robinsohn und die Weiterentwicklungen der Arbeitsgruppe Bildungsforschung am Max-Planck-Institut in Berlin, aber auch auf Vorlagen von Taba oder Tyler aus den USA..$^{6}$ Isenegger bemerkte zu diesen neuen Zugängen zur Konstruktion von Curricula, dass sie sich von den bisherigen hermeneutischen Strategien Klafkis oder Wenigers gänzlich unterscheiden würden, aber eher «globale Postulate» als «geklärte Anweisungen» vorzuweisen hätten. ${ }^{97}$ Isenegger entwarf dann curriculumtheoretisch begründete Schritte zur Konstruktion von Curricula. In einem zweiten Schritt legte er eine Methodologie zur Lernzielerhebung für ein Curriculum in der Lehrerbildung vor.

Joseph Eigenmann vermerkte zur Provenienz seines Doktoratsthemas in seiner Publikation einleitend, sie sei im Rahmen des von der FAL durchgeführten Projektes «Entwicklung und begleitende Analysen eines Curriculums für die Primarschulen» (EBAC) entstanden, und: «Professor Dr. Karl Frey verdanke ich die Problemstellung. ${ }^{98}$ Wie der Untertitel der Arbeit verspricht, beschäftigte sich Eigenmann mit dem «Problem der Sequenzenbildung in der Curriculumkonstruktion unter besonderer Berücksichtigung kognitionspsychologischer Theorien und des Lernzielansatzes».99 Konkret ging er der Frage nach, wie operationalisierte Lernziele zu sequenzieren, also in eine Reihenfolge zu bringen sind, damit schulische Lernprozesse in einem Curriculum sichtbar, für Lehrpersonen nachvollziehbar und letztlich für die schulischen Lernprozesse

92 Horn, 1972, S. 9.

93 Bloom und Krathwohl, i964.

94 BIVO ist ein Akronym für «Bildungsbedürfnisse der Volksschullehrer». Das Projekt war angesiedelt im Bereich der Lehrerbildungsreform und lag in der Hauptverantwortung des Pädagogischen Seminars der Universität Zürich. Weiterführend Isenegger und Gehrig, I97I.

95 Isenegger, 1972.

96 Robinsohn, I967; Knab, I969; Taba, 1962; Tyler, I949.

97 Isenegger, 1972, S. I4.

98 Eigenmann, 1975, S. I 2.

99 Ebd. (Untertitel). 
dienlich werden können. Die Arbeit konzentrierte sich primär auf die Untersuchung und Diskussion bestehender Modelle aus der Psychologie, der Didaktik und der Curriculumforschung.

Urs Peter Lattmanns Dissertation zu Lernzielen und Unterrichtsvorbereitung $^{100}$ nahm Probleme rund um den lernzielorientierten Unterricht in den Blick mit dem Ziel, eine Grundlage für die Entwicklung eines Modells zur lernzielorientierten Unterrichtsvorbereitung (LUV) zu erarbeiten. ${ }^{\text {Ior }} \mathrm{Er}$ ging aus von unterschiedlichen Elementen von Lernzielen: ihnen inhärenten Komplexitätsstufen, zu berücksichtigendem Vorwissen der Schülerinnen und Schülern oder den emotionalen, affektiven oder motorischen Lernbereichen, die angesprochen werden sollten. Mit Referenz auf das Projekt EBAC-PS und die Untersuchung von Santini, wonach Lehrpersonen konkretere, detailliertere Lehrpläne wünschten, ${ }^{102}$ entwickelte Lattmann ein LUV-Modell, welches er ein Jahr später infolge einer Weiterbildungsveranstaltung für Lehrpersonen unter dem Titel «Unterrichtsvorbereitung» im Comenius-Verlag auch publizierte. ${ }^{103}$

Kurt Bossart befasste sich in seiner empirischen Dissertation mit denjenigen Akteuren, die Lernziele formulierten oder, wie es der Titel seiner Arbeit verspricht, mit den Persönlichkeitsmerkmalen von «Abnehmer[n], Experten, Ausbildner[n] und Berufsträger[n] als Formulierer[n] von Lernzielen». ${ }^{104}$ Er stellte sich «die generelle Frage, welche von diesen 4 Personengruppen sich für die gestellte Aufgabe am besten eignet». ${ }^{\text {Ios }}$ Im Rahmen des FAL-Projektes mit dem Titel «Entwicklung und begleitende Analysen des Curriculums Weiterbildungsschule Zug» (EBAC-WS) hat Bossart inhaltsanalytisch die in diesem Projekt entworfenen Lernziele ausgewertet und mit Persönlichkeitsmerkmalen der Beteiligten aufgrund statistischer Berechnungen in Verbindung gebracht.

\section{Schulreform und Curriculumforschung}

«Bildungsplanung ist in der Schweizerischen Bildungspolitik ein relativ neuer Begriff», ${ }^{106}$ hielt Iwan Rickenbacher einleitend in seiner Dissertation fest. Er beschäftigte sich anlässlich seines Engagements im 1968 lancierten Entwicklungsprojekt der Volksschulen im Kanton Schwyz mit Ansätzen und Verfahren der Bildungsplanung. Der Kanton sah sich in Anbetracht interkantonaler Harmonisierungsbestrebungen vor der Aufgabe, etliche strukturelle und curri-

I00 Lattmann, I97Ib.

IOI Ebd., S. I3.

102 Santini, I97I.

I03 Lattmann, 1972.

I04 Bossart, 1975.

I05 Ebd., S. I 5 .

I06 Rickenbacher, 1972, S. 5. 
culare Änderungen an seiner Volksschule zu erwägen. ${ }^{107}$ In seiner Arbeit konzentrierte sich Rickenbacher auf Probleme der Systemanalyse, die Herausforderungen im Findungsprozess von politischen Zielen sowie auf die Umsetzung und Kontrolle geplanter Schulreformen.

Ebenfalls im Forschungsbereich der Schulreform legte 1974 Kurt Aregger eine Dissertation vor, just zu einem Zeitpunkt, «in dem schulnahe, praxisbezogene und lehrerzentrierte Curriculumreformen am intensivsten gefordert werden», ${ }^{108}$ wie Karl Frey im Vorwort der Publikation bemerkte. Aregger legte den Fokus seiner Studien auf die Revision von Curricula unter besonderer Berücksichtigung der Lehrpersonen, zumal «eine grosse Zahl von Lehrergruppen in irgendeiner Form an neuen Unterrichtsinhalten, Lernzielen oder Curricula für einzelne Schulen, Schularten oder -stufen» mitarbeiteten. ${ }^{109}$ Lehrpersonen sollten in der gesamten Phase einer Reform, von der Planung über die Entwicklung bis zur Evaluation eines Curriculums, massgeblich mit in den Reformprozess einbezogen sein. $\mathrm{Zu}$ diesem Schluss kommt Aregger auf der Grundlage einer vertieften Analyse des EBAC-PS-Projektes.

Lehrpersonen und Schulreformen machte auch Ueli Heiniger zum Gegenstand der Forschung. Er publizierte 1977 seine Dissertation zur Neuentdeckung der Rolle der Lehrpersonen mit dem Untertitel «Didaktische Zentren als Marktplatz für Schulreformen». ${ }^{10}$ Anhand von halbstandardisierten Interviews in englischen Teacher's Centres sammelte Heiniger Erfahrungen mit einer für Schulhäuser in der Schweiz noch neuen Organisationform, welche er dann in einem Modell für eine kooperative Schulreform zu pädagogischen Zentren weiterentwickelte. Dem Curriculum kommt nach Heiniger in Schulzentren eine grosse Bedeutung zu als «Informations- und Verständigungsbasis für alle an der Schule Beteiligten». ${ }^{111}$

Das Dissertationsprojekt von Albin Niedermann «stellt den Versuch dar, ein Verfahren zur Entwicklung eines Evaluationsplanes für eine neue Schule zu gewinnen und teilweise erfahrungswissenschaftlich zu erproben». ${ }^{112}$ Er bemerkte einleitend, dass sein Forschungsgegenstand in den USA zur Curriculumevaluation gehöre, dass in Europa in der Regel von wissenschaftlicher Begleitforschung insbesondere bei Schulversuchen die Rede sei, bezüglich der Evalua-

Die EDK legte Ziele zur Harmonisierung der kantonal teilweise sehr unterschiedlichen Schulstrukturen vor, infolge dessen im Kanton Schwyz insbesondere die Oberstufe der Volksschule reorganisiert und ausgebaut werden sollte. Weiterführend Manz, 20I I.

I08 Aregger, 1974, S. 8.

I09 Ebd., S. I7.

I Io Heiniger, 1977.

I I I Ebd., S. I42.

I 2 Niedermann, 1977, S. I 2. 
tion einer gesamten Schule aber ein Forschungsdesiderat bestehe. ${ }^{113}$ Anlässlich eines FAL-Projektes (EBAC-WS) setzte er sich insbesondere mit Problemen der Evaluation in einem wissenschaftlich begleiteten Schulprojekt auseinander und thematisierte Erfahrungen aus der Zusammenarbeit mit involvierten Lehrpersonen und anderen Leuten aus der Praxis.

\section{Lehrberuf und Curriculumforschung}

Bruno Santini verwendete im Vorwort seiner Dissertation folgendes Zitat eines Schulinspektors, um auf sein Erkenntnisinteresse hinzuführen: «Die meisten Lehrer, mit denen ich arbeite, haben eine Einführung in den Lehrplan erhalten. Aber viele von ihnen kommen in Schwierigkeiten, wenn es gilt, die erworbenen Kenntnisse in die Schulwirklichkeit zu übertragen. Der Graben zwischen Theorie und Praxis ist zu gross. ${ }^{114}$ Santini interessierte sich für die hier angesprochene Diskrepanz zwischen curriculumtheoretischen Überlegungen auf der einen Seite und der tatsächlichen Verwendung des Lehrplanes im Schulalltag auf der anderen Seite. Wenn der Lehrplan nicht zuletzt durch schulstrukturelle Neuerungen wie die Gesamtschulen an Bedeutung gewinne, so müsse auch Klarheit über die Stellung der Lehrpersonen zum Lehrplan herrschen. Mittels kooperativer Interviews und Fragebogen ermittelte Santini die Nutzung der bestehenden Lehrpläne durch die Lehrpersonen, fragte nach der Einführung in den Lehrplan während ihres Ausbildungsganges oder auch nach dem Einbezug bei laufenden Lehrplanreformen. Darüber hinaus formulierte er aufgrund der gewonnenen Daten aus den Befragungen Entwicklungsbereiche für künftige Lehrpläne. Es zeigte sich beispielsweise die Tendenz in den Befragungsresultaten, dass sich Lehrpersonen die künftigen Lehrpläne weit umfangreicher wünschten, angereichert mit Lernzielkatalogen oder Verzeichnissen von Lehrmitteln. ${ }^{115}$

Im Rahmen des Schulreformprojektes EBAC-PS im Kanton Freiburg wurden allgemeine Erziehungsziele erarbeitet, welche dann als Grundlage für fachspezifische Leitideen dienten, worauf wiederum Lehr- und Lernziele für die einzelnen Fächer aufbauten. ${ }^{16}$ Die mit in dieses Projekt eingebundenen Lehrpersonen, genauer deren Weltbilder, machte Joe Brunner zum Gegenstand seiner Dissertation. Brunners Forschung interessierte sich erstens fragebogenbasiert für die «normative Grundkonzeption» beziehungsweise den «Orientierungsrahmen» von Lehrkräften, aufgrund dessen sie Zielvorstellungen für ihre Schülerinnen und Schüler formulieren. ${ }^{117}$ Zweitens stellte er Untersuchungen

II 3 Ebd., S. I 5-I9.

I 4 Santini, I97I, S. 5.

II Ebd., S. I32 f.

I 6 Brunner, I980, S. $20 \mathrm{f}$.

II7 Ebd., S. 23. 
dazu an, inwiefern sich die Resultate einer solchen Untersuchung verändern, je nachdem welche Erhebungsmethoden man anwendet.

Dieselbe sozialwissenschaftliche Forschungsmethodik verwendete Ernst Preisig in seiner Dissertationsschrift, entstanden wie jene von Brunner unter wissenschaftlicher Begleitung von Prof. Meinrad Perrez. ${ }^{118}$ Preisig befragte Lehrpersonen und Eltern anhand von Fragebogen mit dem Ziel, Aussagen zu Auswirkungen und Bedingungen erzieherischer Bekräftigung zu treffen. So prüfte er Hypothesen zum Zusammenhang von positiven leistungsbezogenen Bekräftigungstendenzen und der Schulleistung eines Kindes oder zum Zusammenhang einer negativen Bekräftigungstendenz und der Ängstlichkeit eines Kindes. Mit seiner Forschung knüpfte er an Arbeiten zur verhaltenstheoretisch orientierten Erziehungsstilforschung an, welche bislang vor allem an der Universität Marburg von Edmund Ernst Hermann und Kurt H. Stapf betrieben worden sei. ${ }^{119}$

\section{Fazit zum Forschungsgegenstand der FAL}

Mit Blick auf die Darstellungen der Dissertationsprojekte der Mitglieder der FAL wird bezüglich der Curriculumforschung an der Universität Freiburg offensichtlich, dass weitgehend sozialwissenschaftliche Methoden ihre Forschungspraxis bestimmten. Die Forschenden machten sich vertraut mit Erhebungsmethoden wie Fragebogen oder Interviews. Die daraus hervorgehenden Resultate haben sie oftmals durch statistische Verfahren gewonnen, wozu sie Computeranlagen nutzten. Die Universität Freiburg hatte die erforderliche IT-Infrastruktur noch nicht anzubieten, weshalb die FAL-Mitarbeiter auf die Zusammenarbeit mit anderen Forschenden mit Zugang zu solchen Anlagen angewiesen waren. ${ }^{120}$

Auf der Grundlage des Verzeichnisses für Habilitationen und Dissertationen hat Lucien Criblez eine quantitative Analyse der erziehungswissenschaftlichen Promotionen in der Schweiz vorgenommen. ${ }^{121}$ Für die 1970er-Jahre konnten insgesamt 94 Dissertationen gezählt werden. Wie hier gezeigt, haben davon allein die Mitglieder der FAL i3 Dissertationen erarbeitet. Dieser hohe Anteil einer Forschergruppe an der Grundlagenforschung ist bemerkenswert.

I 8 Meinrad Perrez übernahm i975 den deutschsprachigen Lehrstuhl für Pädagogik an der Universität Freiburg von Ludwig Räber, welcher auf Initiative der katholischen Kirche wieder das Rektorat der Stiftsschule Einsiedeln übernahm. Weiterführend Ruhstaller, 1977 .

I 19 Preisig, I980, S. 5.

I 20 Insbesondere die folgenden Mitglieder machten für ihre Qualifikationsarbeiten von grossen Computeranlagen Gebrauch: Bossart, 1975; Horn, 1972; Preisig, 1980; Rickenbacher, 1972; Santini, I97I.

Criblez, $200 \mathrm{Ib}$. 
Anhand der Dissertationen der Mitglieder der FAL wird weiter ersichtlich, dass die Promotionsprojekte mit wenigen Ausnahmen an laufende Reform- und Entwicklungsprojekte in unterschiedlichen Kantonen geknüpft waren. Die Forschenden entwickelten oftmals ihr Erkenntnisinteresse für die Dissertationsprojekte in der Auseinandersetzung im Praxisfeld. Schön ersichtlich wird dieser Sachverhalt am Projekt EBAC-PS zur Erarbeitung eines Curriculums für Primarschulen: Es war angelegt nach den neusten Erkenntnissen der Curriculumforschung und ist selbst aus unterschiedlichen Perspektiven zum Gegenstand der Forschung geworden. Forschung und Entwicklung im Praxisfeld waren eng gekoppelt. Dass es sich bei diesem Austausch gar um einen wechselseitigen Prozess handelte, werden die Ausführungen unten zur Forschungspraxis der FAL zeigen.

An den schulreformspezifischen Projekten der FAL-Mitarbeiter zeigt sich noch etwas Weiteres: Indem die Forscher laufende Reformen wie die Entwicklung eines neuen Schultypus oder die Neugestaltung des obligatorischen Schulwesens im Kanton Schwyz beforschten, erarbeiteten sie Wissen darüber, wie eine Reform zu planen, durchzuführen und zu evaluieren sei. Sie experimentierten mit partizipativen Reformmodellen zum Einbezug verschiedener schulnaher Akteure und grenzten sich so auch ein Stück weit von den Vorschlägen Robinsohns ab. ${ }^{122}$ Curriculumforschung produzierte nebst den bekannteren und in der Lehrerschaft umstritteneren unterrichts- und lehrplanspezifischeren Modellen und Theorien auch Schulorganisations- und Reformwissen. ${ }^{123}$

Zudem zeigen die Darstellungen, dass der Lehrberuf einen wichtigen Bestandteil im Forschungsportfolio der FAL-Mitglieder ausmachte. Lehrerinnen und Lehrer wurden beispielsweise nach ihren Ansichten und Präferenzen oder nach ihren Gewohnheiten bei der Nutzung von Lehrplänen gefragt. Gerade weil Lehrpersonen in die Reformprojekte mit einbezogen waren, wurden ihre Vorstellungen, Ideale und Ansichten für die FAL-Mitglieder interessant, zumal sie in die Planung und Durchführung von Unterricht einfliessen würden, und damit in der Formulierung von Lernzielen relevant waren. Curriculumforschung im Sinne der FAL blieb nicht bei der inhaltlichen Neukonzeption von Lehrplänen stehen. Zusammen mit den Forschungsergebnissen zu Bildungsreformen und zielgerichteter Unterrichtsplanung erarbeiteten sich die Curriculumforscher eine solide Grundlage für spätere Vorstösse zur Erneuerung der Lehrerbildung oder der Lehrerweiterbildung.

I22 Robinsohn schlug vor, «Fachwissenschaftler, «Repräsentanten der wichtigsten Verwendungsbereiche für das Gelernte» sowie «Vertreter der anthropologischen Wissenschaften» als Instanzen einer Curriculumrevision beizuziehen. Robinsohn, 1967, S. 49.

I23 Zu den teilweise gravierenden Differenzen zwischen Lehrpersonen und Curriculumforschern siehe Kapitel 4.3. 
Die Ausführungen zur Forschung der FAL-Mitglieder vermitteln das Bild einer ziemlich breiten thematischen Ausrichtung, deren verbindendes Element erstens in der Anbindung an Curriculumreformprojekte bestand, zweitens in der weitgehend empirischen Arbeitsweise. Drittens liegen über alle Arbeiten hinweg gemeinsame Bezüge zu Theorien und Modellen angelsächsischer Provenienz vor, welche sogleich genauer dargelegt werden.

Eindeutig sind die Bezüge der FAL-Arbeiten im Bereich der Lernziele. Zu ihrer Bedeutung im Rahmen der Curriculumkonstruktion schrieb Lattmann: «Die Curriculumkonstruktion der FAL unterscheidet sich in diesem Punkt grundsätzlich von bisherigen Lehrplänen, indem sie nicht von Themen ausgeht, sondern operationalisierte Lernziele als Ausgangspunkt für Curriculumkonstruktion und detaillierte Lernplanung verwendet.» ${ }^{124}$ Diese Operationalisierung, also die Festlegung eines angestrebten Endverhaltens, der unterstützenden Instrumente bei der Bearbeitung von Aufgaben und des Beurteilungsmassstabes, wurde in den Arbeiten der FAL durchgängig mit der Publikation von Robert Mager zu Lernzielen und programmiertem Unterricht ${ }^{125}$ legitimiert. Zur Klassifizierung der Lernziele nach ihren Anforderungen wurde auf ein Taxonomiemodell aus der Literatur der Psychologie zurückgegriffen, in der Regel auf Bloom, ${ }^{126}$ aber auch Gagné, Bruner und Ausubel als Vertreter der lernpsychologischen Forschung ${ }^{\mathrm{I} 27}$ wurden regelmässig zitiert. Goodlad, Tyler und, als einziger Forscher ohne amerikanischen Hintergrund, Robinsohn bilden einen weiteren gemeinsamen Bezugspunkt für die Arbeiten der FAL. ${ }^{128}$ An ihnen orientierten sie sich bei Überlegungen zur Curriculumkonstruktion, allerdings ohne ihre Modelle diskussionslos auf den eigenen Anwendungsbereich zu übertragen.

\subsubsection{Die Forschungspraxis der FAL}

Die wissenschaftsgeschichtliche Forschung tendiert derzeit dazu, mit Bezug auf kulturgeschichtliche Zugänge die Prozesse der Wissensgenese nachzuzeichnen, also Produktionsformen und dabei involvierte Akteursgruppen in den Blick zu nehmen. ${ }^{129}$ Schaut man sich demgemäss die Tätigkeiten der FAL-Mitglieder genauer an, lassen sich grob vier Schwerpunkte ausmachen: Die Mitarbeiter

I24 Lattmann, I97Ib, S. 6r.

I25 Mager, I965.

I 26 Bloom und Krathwohl, i964.

I 27 Ausubel, I968; Bruner, I966; Gagné, I965.

I 28 Goodlad, I964; Robinsohn, I967; Tyler, I949.

I 29 Vgl. dazu Baur, Besio, Norkus und Petschick, 2016; Brandstetter, 201 2; Breidbach, 2007; Reichmann und Knorr Cetina, 20 I6; Sarasin, 20 I I. 
schrieben in der Regel an einer Doktorarbeit, sie waren aktiv in Curriculumprojekten, die entweder vom SNF oder einem anderen Auftraggeber finanziert waren, sie publizierten in Fachzeitschriften und sie waren oft in die Lehrtätigkeit an der Universität Freiburg eingebunden. Ausgehend davon werden in diesem Teilkapitel vier Aspekte hinsichtlich des forschungspraktischen Handelns der Freiburger Arbeitsgruppe für Lehrplanforschung diskutiert: die Interdisziplinarität innerhalb der FAL, ihre selbstreferenzielle Publikationspraxis, die Veränderungen der Forschungspraxis und der Forschungsschwerpunkte und schliesslich das Verhältnis der FAL zu empirischen Forschungsmethoden.

\section{Interdisziplinarität in der FAL}

Die Arbeitsweise der FAL muss im Kontext einer neueren Erscheinung im praktischen Forschungshandeln gesehen werden, die in den einleitenden Worten im Jahresbericht des Nationalfonds von 1967 wie folgt umschrieben wurde: «Es wird immer schwieriger, sich über die Entwicklung eines Gebietes umfassend zu orientieren, man ist gezwungen, sich immer mehr zu spezialisieren, verliert den Überblick und kann nur noch in Gruppen oder ‘Teams` ein Gebiet erfolgreich bearbeiten. Ja, es ist gar nicht abwegig, von einer eigentlichen Industrialisierung der Forschung zu sprechen. $»^{130}$

Die Tendenz, in Gruppen an Forschungsthemen zu arbeiten, führte indes dazu, dass beim SNF zunehmend grössere Geldbeträge für Projekte beantragt wurden. Der SNF erklärte sich das damit, dass «namentlich in den modernen Disziplinen, der Soziologie, der Politologie, den Wirtschaftswissenschaften und der Bildungsforschung immer mehr im Teamwork gearbeitet wird». ${ }^{131}$ Die Mitglieder der FAL als dominante Repräsentanten der Curriculumforschung in der Schweiz machten es sich zur Aufgabe, Forschungsstränge insbesondere aus angloamerikanischen Zusammenhängen der «curriculum studies», der Psychologie, der Innovationsforschung und teilweise des programmierten Unterrichts zusammenzuführen und gemäss den Anwendungszusammenhängen des Bildungswesens in der Schweiz zu übersetzen. ${ }^{132}$ Dazu waren sie, wie der Nationalfonds beobachtet hat, als Team organisiert, mit zeitweise über zehn gleichzeitig angestellten Mitarbeitern, die sich unter dem Prädikat «Curriculumforschung» schweizweit Bekanntheit verschafften.

Die einzelnen Mitglieder der FAL waren Spezialisten für je einen bestimmten Bereich, bedingt durch die vertiefte Beschäftigung mit einem Promotionsthema, aber auch durch unterschiedliche Studienrichtungen und berufliche Tätigkeiten vor dem Engagement in der FAL. Mit Urs Isenegger und Kurt Schmid

I30 Jahresbericht SNF 1967, S. I I, StAZH, DSP 49.

I 3 I Jahresbericht SNF i97I, S. 43, StAZH, DSP 49.

I32 Vgl. Höhener und Criblez, 2018. 
wirkten studierte Psychologen mit, Ralf Horn hingegen war in statistischen Belangen besonders beschlagen. Joseph Eigenmann und Urs Peter Lattmann waren eher wissenschaftstheoretisch und hermeneutisch orientiert, Kurt Aregger und Iwan Rickenbacher wiederum legten den Schwerpunkt auf Innovations- und Planungsarbeiten. ${ }^{133}$ Diese exemplarische Darstellung unterschiedlicher Schwerpunkte und Spezialbereiche der Mitglieder der FAL präsentiert die Arbeitsgruppe als interdisziplinär ausgerichtetes Team. Mit Blick auf ihre Publikations- und Projekttätigkeit wird darüber hinaus deutlich, dass ihre Arbeiten sehr oft im Verbund entwickelt und realisiert worden sind.

\section{Selbstreferenzielle Curriculumforschung}

Im Tätigkeitsbericht der FAL für das Jahr 1973 sind 32 Publikationen aufgeführt, die in unterschiedlichen Formaten erschienen sind. ${ }^{134}$ Publiziert wurde in verschiedenen schulnahen Zeitschriften wie der «Schweizerischen Lehrerzeitschrift» oder der «Schweizer Schule», in Sammelbänden, in wissenschaftlichen Zeitschriften wie dem «Journal of Curriculum Studies», in der katholischen Zeitschrift «Civitas» oder in der eigenen Reihe mit dem Titel «Studien und Forschungsberichte aus dem Pädagogischen Institut der Universität Freiburg/ Schweiz». Von den 32 publizierten Beiträgen wurde knapp die Hälfte von einem FAL-Autorenkollektiv geschrieben. Im darauffolgenden Jahr wurden in neun von 2I Fällen wiederum Autorenteams in unterschiedlichen Zusammensetzungen gebildet. ${ }^{135}$ Die Mitglieder der FAL haben oft gemeinsam publiziert, aber selten sind Publikationen mit FAL-externen Forschenden entstanden.

Die enge Zusammenarbeit zwischen den Mitgliedern hat dazu geführt, dass sie untereinander gut über die jeweiligen Forschungsschwerpunkte informiert waren. Die gemeinsame Erarbeitung von Publikationsbeiträgen oder Zwischenresultaten in laufenden Projekten kommt einer stetigen Weiterentwicklung der Curriculumforschung gleich oder, mit kuhnschen Worten, einer intensiven Bearbeitung der normalen Wissenschaft. ${ }^{136}$ Davon zeugt auch die gegenseitige Bezugnahme in Publikationen.

Ein anschauliches Beispiel hierfür präsentiert die weitere Entwicklung eines Befundes aus Bruno Santinis Forschung zu Ansprüchen der Lehrerinnen und Lehrer an künftige Curricula. Die Hälfte der von Santini anlässlich seiner Dissertation befragten Lehrinnen und Lehrer wünschte «einen Bildungsplan, d. h.

I33 Lattmann, 2015.

I 34 Forschungszentrum FAL, Tätigkeitsbericht 1973, Freiburg, 21. I. 1974, Archivalien der FAL.

I35 Forschungszentrum FAL, Tätigkeitsbericht 1974, Freiburg, Februar 1975, Archivalien der FAL.

I36 Kuhn, 1967 . 
ein Vollcurriculum. Diese Form umfasst neben den Komponenten des Stoffplans mit Auswahlstoffen Angaben über Prüfverfahren, Aufgabe und Stellung des Lehrers, wissenschaftliche und methodische Erläuterungen der Themen.» ${ }^{137}$ Eine Wiederaufnahme dieser Aussage nahm Isenegger wenig später in einem Artikel für die «Schweizerische Lehrerzeitung» vor, ${ }^{13^{8}}$ Rickenbacher bezog sich in seinem Beitrag zum «Lehrer als Objekt wissenschaftlicher Untersuchung» ebenfalls darauf. ${ }^{139}$ Dann erschien im Jahrbuch der Schweizerischen Konferenz der kantonalen Erziehungsdirektoren mit dem Schwerpunkt «Schulkoordination über Lehrplanreform» von Santini, Urs Isenegger und Hans Stricker ${ }^{\mathrm{r} 40}$ ein Beitrag zur «Form und Inhalt künftiger Lehrpläne». ${ }^{\text {III }}$ Darin wurde informiert über die Elemente, mit welchen inskünftig die Lehrpläne versehen werden sollten. Die einstigen Forschungsresultate von Santini lagen nun als bildungspolitischer Vorschlag vor. Wie dieses kleine Beispiel zeigt, stützten sich die Mitglieder der FAL gegenseitig auf ihre Forschungsresultate ab, sie verwiesen auf Erkenntnisse aus Studien ihrer Kollegen, etwa zur Legitimation weiterführender Fragestellungen, und sie erreichten selbst bildungspolitische Kreise mit ihren empirischen Befunden.

\section{Wandel der Forschungsschwerpunkte}

Der Blick auf die Forschungspraxis der FAL - gewissermassen auf die Mikroebene der wissenschaftlichen Wissensproduktion - ermöglicht es, die Rezeption, Verarbeitung und Weiterentwicklung von Wissensbeständen innerhalb einer grösseren Arbeitsgruppe zu beleuchten. ${ }^{\mathrm{I} 22}$ Werden demgemäss die Publikationen der FAL in diachroner Weise betrachtet, zeichnet sich eine bestimmte Verlagerung der Forschungsschwerpunkte ab. Armin Gretler hat hierzu bemerkt, die FAL habe während ihrer Schaffenszeit zweimal einen thematischen Wandel vollzogen: In einer ersten, vorbereitenden Phase habe die FAL einige

I37 Santini, I97I, S. I I I.

I 38 Isenegger, 1973, S. 94.

I39 Rickenbacher, 1974, S. 548.

I40 Hans Stricker war zunächst Schulinspektor im Kanton Bern und ab I97I und für die nächsten 20 Jahre Gründer und Direktor des Amtes für Unterrichtsforschung des Kantons Bern.

I4 I Stricker, Isenegger und Santini, 1978.

I42 Baur et al., 2016. Reichmann und Knorr Cetina, 20I6, vertreten den Standpunkt, dass Wissenschaft und Wissensproduktion als Mehrebenensystem zu begreifen und zu analysieren ist. Während auf der Makroebene unterschiedliche Disziplinen in der Wissenschaft oder deren Verhältnis zu anderen gesellschaftlichen Feldern zu erforschen seien, stehe auf der Mesoebene die Frage im Zentrum, wie Forschung organisiert werde, etwa an Universitäten. Auf der Mikroebene wird auf den Arbeitsalltag von Forschenden geachtet. Dabei stehen Wissenskulturen und die Praktiken der Wissenserzeugung, Wissensvalidierung und Wissensevaluation im Fokus. 
vergleichende Überblickswerke zu den bestehenden kantonalen Lehrplänen in der Schweiz vorgelegt, um in einer zweiten Phase Curriculumforschung «im engeren Sinn» zu betreiben. ${ }^{143}$ Ab 1975 sei die FAL dann auf Fragen der Didaktik und der Wirksamkeit von Unterricht umgeschwenkt und habe «wissenschaftstheoretische Grundlagenprobleme im Bereich der Erziehungs- und Unterrichtsziele» bearbeitet. ${ }^{144}$ Für den hier angesprochenen Wandel war unter anderem die Rolle und Funktion der Schulreformprojekte unter der Leitung der FAL entscheidend.

Das EBAC-PS-Projekt der FAL ist als Bezugspunkt für die Dissertationen von Joseph Eigenmann, Bruno Santini, Kurt Aregger und Joe Brunner bereits genannt worden. ${ }^{\mathrm{I} 5}$ Es wurde argumentiert, dass die Forschenden dieses Schulreformprojekt im Kanton Freiburg für ihre Dissertationsprojekte genutzt haben zur Erarbeitung von Fragestellungen oder als Gelegenheit, empirische Daten durch Befragungen zu generieren, was von der FAL auch in Bezug auf laufende Drittmittelprojekte beschrieben wurde: «Die EBAC-Projekte stellen die Erfahrungsfelder für die mehr auf Theoriebildung, auf übergreifendere, allgemeinere Fragen ausgerichteten Forschungsziele des Nationalfonds-Projektes dar.» ${ }^{146}$

Bis 1975 waren mit Ausnahme des EBAC-PS-Projektes alle weiteren Reformprojekte ${ }^{147}$ der FAL entweder ausgelaufen, bereits in der Endphase, oder sie waren vom mittlerweile gegründeten Zentralschweizer Beratungsdienst für Schulfragen (ZBS) übernommen worden. Entsprechend dieser sich abzeichnenden Zäsur im Bereich der Curriculumentwicklungsprojekte ist den Mitgliedern der FAL ein wichtiger Bezugspunkt für die Akquise wissenschaftlicher Fragestellungen entgangen.

Nach 1975 sind die Dissertationen von Ueli Heiniger, Albin Niedermann, Joe Brunner und Ernst Preisig entstanden. Sie beschäftigen sich mit didaktischen Zentren in Schulen, Evaluationskonzepten, Erziehungsverhalten und Erziebereits als relevante Einflussgrösse hinsichtlich der Forschungsleistungen der Gruppe
thematisiert.

I46 Forschungszentrum FAL, Tätigkeitsbericht 1974, S. I0, Freiburg, Februar 1975, Archivalien der FAL.

I47 Die FAL-Mitglieder engagierten sich im «Entwicklungsplan für die Schulen im Kanton Schwyz», in dessen Folgeprojekt zur «Entwicklung und begleitenden Analysen des Curriculums der Oberstufe Schwyz», in der «Entwicklung und begleitenden Analyse eines Curriculums für die Weiterbildende Schule Zug», entwarfen ein «Curriculum Religionsunterricht» und waren beteiligt am Projekt $\mathrm{zu}$ «Bildungsbedürfnissen für Volksschullehrer». 
hungszielen. Die Auseinandersetzung mit Curricula war abgesehen von Brunners Arbeit stark in den Hintergrund gerückt.

Die Arbeit von Heiniger und Brunner können aber noch in einem anderen Zusammenhang gelesen werden: Bis Mitte der I970er-Jahre hatten die FALMitglieder bereits etliche Jahre Erfahrungen sammeln können in den unterschiedlichsten Projekten zur Schulreform. Sie waren konfrontiert mit «Hoffnungen, Befürchtungen, Widerstand und Resignation» ${ }^{14^{8}}$ der Involvierten, insbesondere von Lehrpersonen. Die Forscher stürzten sich zunächst in die Entwicklung von Lernzielen mit den Freiburger Lehrpersonen, um im Nachhinein zu konstatieren, dass die Erarbeitung von Lernzielen eine Klammer in Form von Leitideen oder Erziehungszielen benötigen würde. ${ }^{149}$ Die Dissertationen von Heiniger und Brunner nahmen unterschiedliche Erfahrungen aus den Reformprojekten auf und stehen für neue Aspekte der einst konzipierten «Lehrplanreform als Grundlage der Schulreform», ${ }^{150}$ wie sie von der FAL zu Beginn der I970er-Jahre entworfen wurde. Diese beiden wie andere projektbezogene Dissertationen aus der Feder der Mitarbeiter der Freiburger Arbeitsgruppe zeigen deutlich, wie sich wissenschaftliche Fragestellungen durch die langjährige Auseinandersetzung mit Reformprojekten veränderten und teilweise erst dadurch entwickelt werden konnten. ${ }^{\text {ss }}$

Ersichtlich ist auch der erfahrungsbedingte Wandel der Forschungsschwerpunkte der FAL an der Umbenennung der beim Nationalfonds eingeworbenen Drittmittelprojekte. Das erste Projekt von 1972 trug den Titel «Methoden der Curriculumkonstruktion». Die Verlängerung dieses Projektes trug ab 1975 den Titel «Struktur und Funktion didaktischer Bezugssysteme für die Unterrichtsplanung». Ebenfalls ab 1975 arbeiteten die Mitarbeiter der FAL an einem Projekt mit dem Namen «Problemaspekte der Leitideen in der Volksschule empirische Untersuchung im Kanton Freiburg». Das erfolglos eingereichte Projekt mit dem Titel «Integrierte Schulentwicklungsplanung» ${ }^{152}$ ist ebenso symptomatisch für die Neuausrichtung der Forschungsschwerpunkte der FAL $\mathrm{ab}$ 1975. ${ }^{153}$

I48 Heiniger, I977, S. 20.

I49 Brunner, I980, S. 20 f.

I 50 Aregger, Brunner und Isenegger, 1972.

I 5 I Urs Isenegger hat diesen Sachverhalt rückblickend als «fortlaufenden Lernprozess» bezeichnet. Vgl. Isenegger, 2016.

I52 Protokoll der ordentlichen FAL-Generalversammlung 1975, 4. 6. 1975, Archivalien der FAL.

I 53 Über einzelne Teilforschungsbereiche innerhalb der jeweiligen Nationalfondsprojekte der FAL weiterführend Aregger und Gretler, 1983, S. 826 f. 


\section{Empirisches Forschen in der Pädagogik}

In seiner Antrittsvorlesung im Juli 1962 vertrat Heinrich Roth ${ }^{154}$ zur Frage der realistischen Wendung in der pädagogischen Forschung den Standpunkt: «Die Fortschritte der erfahrungswissenschaftlichen Methoden in allen Wissenschaften vom Menschen, die Variabilität der Methoden, der Verzicht auf einen falschen Exaktheitsfanatismus zugunsten einer den Gegenstand einkreisenden Methodenvielfalt und einer Strategie der Methoden, die laufende kritische Selbstkontrolle der Forschenden, der Prozess von Forschung, Kritik und Kontrolle (Wittram), die Einsicht in den Wert von Theorien sogenannter <mittlerer Reichweite, haben es auch in der Pädagogik möglich gemacht, Wirklichkeit auf Wahrheit zu befragen.» ${ }^{155}$

Die Mitglieder der FAL haben weitgehend in Roths Sinne ihre Forschungstätigkeit auf «erfahrungswissenschaftliche» Methoden abgestützt. Die bisherigen Lehrpläne wurden grundlegend hinterfragt und relativiert. Mit sozialwissenschaftlichen Studien rückten die Mitglieder der FAL dem Lehrplan zu Leibe und fragten danach, was zu lernen sei, wie das überprüft werden könne und nach welchen Methoden das Lernen besser auszurichten wäre. Sie fragten die Lehrpersonen nach ihren Gewohnheiten in der Nutzung von Lehrplänen und begleiteten die Erarbeitung darauf abgestimmter neuer Curricula. Darüber hinaus haben sie bereits 1970 erste Interventionsstudien bei Lehrpersonen durchgeführt und mit statistischen Mitteln ausgewertet. ${ }^{156}$

Die Freiburger Neubesetzung der deutschsprachigen Professur für Pädagogik mit Meinrad Perrez, einem studierten Psychologen, im Jahr 1975 ist symptomatisch für den von Roth beschriebenen Wandel der Pädagogik weg von historisch-hermeneutisch ausgerichteter Forschung und Lehre hin zu empirischsozialwissenschaftlichen Zugängen und Forschungspraxen. Die Dissertationen der Mitglieder der FAL zeigen jedoch, dass bereits in der Amtszeit von Ludwig Räber sozialwissenschaftliche Methoden in Freiburg rege Anwendung gefunden haben. Dies muss einerseits auf Karl Frey und seine Beziehungen zu seinem einstigen Studienort Marburg zurückgeführt werden. Andererseits dürften die Freiheiten, die Karl Frey in der Gestaltung und Begleitung von Dissertationsvorhaben von Räber eingeräumt wurden, eine entscheidende Rolle gespielt haben.

I 4 Heinrich Roth (1913-1992) war Professor für Pädagogik, zunächst in Frankfurt am Main, 196 I-1973 in Göttingen, und gilt als früher Vertreter der empirischen pädagogischen Forschung. Er war ab i965 Mitglied im Deutschen Bildungsrat. Weiterführend Hoffmann, 2005 .

I55 Roth, 1963, S. I19.

I 56 K. Frey und Lattmann, 1970. 
Die Zugehörigkeit von Karl Frey zur 1965 gegründeten Arbeitsgruppe für empirische pädagogische Forschung (AEPF) ${ }^{157}$ der Deutschen Gesellschaft für Erziehungswissenschaft (DGFE) ist ein weiterer Beleg für die deutliche Positionierung in der Frage, wie pädagogische Forschung zu erfolgen habe. Die Mitglieder der AEPF bekannten sich dazu, «die empirische pädagogische Forschung durch Diskussion ihrer Methoden und Forschungsprojekte zu fördern und die Verwirklichung der Forschung durch Beratungen über Projektplanung und -realisierung zu unterstützen». ${ }^{158}$ Die I4. Tagung der AEPF wurde zudem von Karl Frey erfolgreich an der Universität Freiburg (Schweiz) mit einem Schwerpunkt zur Curriculumkonstruktion durchgeführt. Die Tagung verzeichnete mit die höchsten Besuchszahlen in der Geschichte der AEPF bis I990. 59 Sie bot darüber hinaus gute Gelegenheit, mit anderen empirisch Forschenden Kontakte zu knüpfen, zumal Wolfgang Klafki, Uwe Henning oder Karl-Heinz Flechsig ebenfalls zu dieser Gruppe gehörten, später mit Karl Frey zusammenarbeiteten oder sich in Bewerbungsverfahren als Gutachter betätigten.

\section{Fazit zur Forschungspraxis der FAL}

Eine Besonderheit der Organisation der Curriculumforschung in der Schweiz besteht darin, dass ihre Hauptvertreter allesamt an derselben Universität in einer Arbeitsgruppe tätig waren. Die dadurch bedingte Konzentration von themenspezifischem Wissen und einschlägigen Forschungsprojekten macht die Freiburger Arbeitsgruppe zu einem anschaulichen Fall für die Entwicklung einer Forschungsrichtung innerhalb der pädagogischen Forschung, die weitgehend mit hermeneutischen Arbeitsweisen gebrochen hatte und mit empirischen Methoden vorging. Unter dem Schlagwort «Curriculum» knüpften die Mitglieder der FAL Fäden aus Didaktik, Lernpsychologie, Organisationstheorie und Erziehung zu einem neuen Strang und publizierten ihre Erkenntnisse breit in der fachwissenschaftlichen Literatur. Dass die einzelnen Mitglieder je eigene Forschungsschwerpunkte und -interessen pflegten, trug zum Erfolg der Arbeiten bei. Die Mitglieder standen in engem gegenseitigem Austausch, erarbeiten Resultate oftmals gemeinsam und vertraten gemeinsame Positionen in wissenschaftlichen Debatten. ${ }^{160}$

Darüber hinaus ist die Arbeitsweise der FAL ein anschauliches Beispiel für eine Art von Forschung, die kaum ohne greifbaren Anwendungsbereich in dieser

I 57 Zur Geschichte der AEPF vgl. Ingenkamp, I992; von Saldern, I992, 20 I0.

I 58 Ingenkamp, I992, S. I IO.

I59 Von Saldern, 1992.

I60 Neben der FAL könnte als vergleichbares, wenn auch älteres Beispiel für eine straff organisierte und längerfristig bestehende Forschungszusammenarbeit in der Pädagogik das Institut Jean-Jacques Rousseau in Genf angeführt werden. Vgl. dazu Hofstetter, Ratcliff und Schneuwly, $20 \mathrm{I} 2$. 
Weise hätte praktiziert und gefördert werden können. Es bedurfte der nebenher von der FAL begleiteten Schulreformprojekte. Sie boten auf der einen Seite Zugang zu empirischen Daten für die Dissertationsprojekte. Sie lieferten Problemstellungen aus der Praxis für die theoretische Bearbeitung. Auf der anderen Seite ermöglichten sie etliche Anstösse für eine thematische Weiterentwicklung der Curriculumforschung weg von Fragen der Lernzielformulierung und deren Evaluation hin zu Fragen der Schulentwicklung oder der übergeordneten Bedingungen und Ziele von Schule, den Leitideen.

Nach zehn Jahren des Forschens an der Universität Freiburg hat sich die Freiburger Arbeitsgruppe 1978 aufgelöst. Das nächste Kapitel diskutiert einige mögliche Erklärungen.

\subsection{Erklärungsansätze für die Einstellung der Curriculumforschung und der FAL}

Im Kern der folgenden Ausführungen steht die zu Beginn dieses Kapitels aufgeworfene Frage: Weshalb wurde die Schweizer Grundlagenforschung im Bereich der Lehrpläne eingestellt? Ganz grundlegend mit Kuhn ${ }^{161}$ gesprochen bestehen zwei Optionen: I. Curriculumforschung hat sich als Paradigma einer Normalwissenschaft gar nie etabliert, oder 2. sie hat zwar vorgelegt, was Kuhn als Kriterium anführt, «anerkannte wissenschaftliche Leistungen, die für eine gewisse Zeit einer Gemeinschaft von Fachleuten massgebende Probleme und Lösungen liefern», ${ }^{162}$ es handelte sich im vorliegenden Fall aber um nicht mehr als ein Strohfeuer, intensiv, aber kurz. ${ }^{163}$

\subsubsection{Kaum Pädagogiklehrstühle für Forschende mit Schwerpunkt Curriculumforschung}

Eine erste quellenbasierte Erklärung dafür, dass die Curriculumforschung in der Schweiz nach der Auflösung der FAL nicht weitergeführt wurde, kann darin gefunden werden, dass in der Schweiz kein einziger Lehrstuhl neu geschaffen wurde, der mindestens teilweise auf Bereiche der Curriculumforschung

I6I Kuhn, 1967.

162 Ebd., S. Iо.

I63 Die vorliegende Referenz auf Kuhn ist im Sinne einer wissenschaftsgeschichtlichen Bezugnahme zu verstehen und soll losgelöst von erkenntnistheoretischen oder wissenschaftstheoretischen oder gar psychologischen Einordnungen verstanden werden. Weiterführend dazu Hoyningen-Huenem, 1989; Richards und Daston, 2016. 
ausgerichtet war. ${ }^{164}$ Wohl befand sich die Bildungsforschung in der Schweiz seit den I960er-Jahren in einer Phase der Neukonstituierung, die Forscher der FAL hatten aufgrund ihres Profils aber kaum Gelegenheit, sich in einem Wahlverfahren erfolgsversprechend zu bewerben. Erfolgreich um eine Professur wegbeworben hat sich nur der Gründer Karl Frey, ans Institut für Pädagogik der Naturwissenschaften in Kiel, bevor er 1988 nach Zürich an die Eidgenössische Technische Hochschule (ETH) berufen wurde. Von den anderen i9 FAL-Mitarbeitern hatten aber durchaus auch andere Ambitionen auf eine Professur.

Im Wahlverfahren 1976/77 zur Berufung auf die Assistenzprofessur an der Universität Freiburg waren auch Repräsentanten der Schweizer Curriculumforschung mit ihren Bewerbungen vertreten. Die Findungskommission hat für die engere Auswahl bestimmte Kriterien für die Stelle formuliert und diese für jeden Kandidierenden durchdekliniert. ${ }^{165}$ Für die Kommission dieser katholischen Universität war die Konfession der Bewerbenden noch Mitte der I970er-Jahre das erstgenannte Selektionskriterium, vor den Französischkenntnissen, der bisherigen Ausbildung, Schulerfahrung oder Erfahrungen in der Lehrerbildung. Aus dem Kriterienkatalog geht zudem hervor, dass für die wissenschaftliche Qualifikation für die ausgeschriebene Assistenzprofessur mit allgemeindidaktischer Ausrichtung die Themenbereiche Schulorganisation und Curriculum weniger gewichtet wurden. Die Diskussionsprotokolle der Kommission machen deutlich, dass eine Promotion in Psychologie einer Promotion in Pädagogik vorgezogen wurde. ${ }^{166}$ Am Schluss des Wahlverfahrens hat der erstplatzierte Bewerber den Ruf der Universität Freiburg abgelehnt. Es wäre dann um ein Haar eine Bewerbung mit Hintergrund aus der Curriculumforschung berücksichtigt worden, wäre nicht noch eine weitere Person zu einem späteren Zeitpunkt auf Anfrage in das Verfahren einbezogen und schliesslich berufen worden. ${ }^{167}$

Erst Mitte der 1980er-Jahre bot sich mit dem frei gewordenen Lehrstuhl von Konrad Widmer in Zürich wieder die Chance auf eine Professur für die Curriculumforschenden, zumindest für jene, die ihre wissenschaftliche Karriere

I64 In Bern wurde I969 ein Lehrstuhl für pädagogische Psychologie geschaffen, I 968 einer in Zürich für Pädagogik und pädagogische Psychologie, 1972 einer in Zürich für Sozialpädagogik. Vgl. Criblez, 2007b.

i65 Tabelle zum Protokoll vom 3 I. 5. 1976, Dekanat der philosophischen Fakultät, Universität Freiburg.

I66 Protokolle der Sitzung vom 30. I0. 1976 und vom I3. I2. 1976, Kommission zur Berufung eines Assistenzprofessors für Allgemeine Didaktik, Dekanat der philosophischen Fakultät, Universität Freiburg.

I67 Protokolle der Sitzung vom I 3. I2. 1976 und vom 23. I2. 1976, Kommission zur Berufung eines Assistenzprofessors für Allgemeine Didaktik, Dekanat der philosophischen Fakultät, Universität Freiburg. 
nicht schon lange aufgegeben hatten. Die Akten des Berufungsverfahrens zeigen, dass die bisherige pädagogisch-psychologische Ausrichtung noch weiter gestärkt werden sollte. ${ }^{168}$ Selbst die vorgängige schriftliche Intervention eines Bewerbers beim Regierungsrat mit der überzeugend begründeten Bitte um Prüfung und Ausweitung der Denomination sollte diesen Kurs nicht beeinflussen, welchem die Wahlkommission ohne Umschweife folgte. ${ }^{169}$

Nicht ganz zufällig wurden hier die Beispiele der aus Sicht des FAL-Personals erfolglosen Wahlverfahren an der Universität Zürich und der Universität Freiburg ausgewählt. Weitere Möglichkeiten einer Bewerbung für eine wieder zu besetzende oder neu geschaffene Professur an einer Schweizer Universität mit einschlägiger Denomination standen gar nicht zur Verfügung. Die Curriculumforschenden mit Ambitionen auf eine Professur befanden sich in den I970er-Jahren in einer unvorteilhaften Situation: Der Ausbau der Professuren für Pädagogik hielt sich sehr in Grenzen. Zudem fehlten Stellen im höheren Mittelbau weitgehend, weshalb Drittmittelprojekte oder Forschungsstipendien für eine Weiterbeschäftigung an der Universität nach der Promotionsphase unerlässlich waren. ${ }^{170}$ Die institutionellen Logiken der Professorenuniversitäten haben sich für die wissenschaftliche Weiterentwicklung des FAL-Personals als grosse Hürde herausgestellt.

\subsubsection{Wirtschaftskrise und Stellenmangel in der Forschung}

I 973 überraschte der erste Ölpreisschock die Volkswirte weltweit, was wenig später auch für die Schweizer Forschungslandschaft Konsequenzen zeigte. ${ }^{17 \mathrm{I}}$ Der Schweizerische Nationalfonds war gar der umgekehrten Ansicht: «[...] die Krise, - wenn man dieses grosse Wort gebrauchen will - hat die Universitäten und die Grundlagenforschung erfasst, bevor sie die Wirtschaft erfasste! ${ }^{172}$ Der Nationalfonds konstatierte 1974 einen Rückgang seiner Fördermittel, ferner empfahl er etlichen Antragstellern, von einer Eingabe abzusehen, und er lehnte ungewöhnlicherweise die Förderung eines Drittels der eingegangenen Anträge ab. ${ }^{173}$

Auch für die Forscher der Freiburger Arbeitsgruppe wurde der wirtschaftliche Wandel spürbar. 1976 berichteten Joseph Eigenmann und Anton Strittmatter,

I68 Fakultätsbericht vom 10. 3. 1986, UAZ, E.3.I.304.

I69 Brief vom 28. 3. I985 an Erziehungsdirektor Gilgen, UAZ, E.3.I.304.

I70 Criblez, 2002.

I7I Graf, 20I4; Sheldon, 2010.

I72 Jahresbericht SNF 1975, S. 8, StAZH, DSP 49.

I73 Jahresbericht SNF 1975, StAZH, DSP 49. 
sie würden hoffen und auch etwas dazu beitragen wollen, dass die FAL noch eine Existenzgrundlage finden könne, zumal «in einer Zeit, da die meisten Menschen sich wieder vermehrt nach Sicherheit und Stabilität sehnen». ${ }^{174}$ Ein Jahr später wurde über eine noch kritischere finanzielle Lage der FAL informiert. Zusätzlich würden der FAL einige durch die Unsicherheiten bedingte personelle Abgänge und die gewachsene Komplexität der Aufgabenstellungen zusetzen. ${ }^{175}$ Im letzten Tätigkeitsbericht wurde dann erwähnt, wie die Versuche einer Institutionalisierung der FAL gescheitert seien. Obwohl die Zusammenarbeit mit dem Kanton Freiburg in unterschiedlichen praxisorientierten Lehrplanprojekten zwar weitergelaufen wäre, konnte die angestrebte Aufnahme der FAL ins Institut an der Universität oder ins Erziehungsdepartement des Kantons Freiburg nicht realisiert werden. ${ }^{176}$

Die wirtschaftlich herausfordernden Bedingungen für die FAL mögen dazu beigetragen haben, dass eine Weiterführung der Arbeiten der FAL erschwert wurde, auch vor dem Hintergrund der Tatsache, dass ihr jüngstes, nationalfondsgefördertes Projekt auslief und kein neues eingereicht wurde. Massgeblich zum Ende der Curriculumforschung in Freiburg und damit der Schweiz scheinen aber die personellen Fluktuationen beigetragen zu haben. Ab Mitte der 1970er-Jahre hat die Personalsituation der FAL wie oben erwähnt verschiedentlich Probleme bereitet. Nur schon die anstehenden Aufgaben zu erledigen und der Lehrverpflichtung nachzukommen war eine Herausforderung. Bereits um 1972, also nach vier Jahren, hatten fünf Mitarbeiter die FAL schon wieder verlassen. Neu dazu gekommen waren ebenso viele, und ein vergleichbarer Wechsel hat sich 1975/76 wiederholt. Bloss vier Mitarbeiter, Joseph Eigenmann, Bruno Santini, Joe Brunner und Ueli Heiniger, waren bis zu acht Jahre in der FAL engagiert. In der Grafik 3 wird ersichtlich, bei welchen Arbeitgebern die Mitarbeiter nach ihrer Zeit bei der FAL tätig geworden sind. Anton Strittmatter etwa trat nach der FAL zunächst beim Zentralschweizer Beratungsdienst für Schulfragen (ZBS) als Leiter die Nachfolge von Iwan Rickenbacher an, bevor er die Leitung des Dachverbandes der Schweizer Lehrerinnen und Lehrer (LCH) übernahm. Generell zeigt die Grafik anhand der Punkte die Tendenz, wohin sich die Forscher beruflich entwickelten: überwiegend hin zur Lehrerbildung und zu kantonalen Verwaltungs- und Planungsstellen. Weder als Seminardirektor noch als Projektbeauftragter für Lehrplanfragen einer kantonalen pädagogischen Abteilung hat ein Grundlagenforscher im Curriculumbereich weitergewirkt.

I74 Forschungszentrum FAL, Tätigkeitsbericht 1975, Freiburg 1976, Archivalien der FAL, S. 2.

175 FAL Information 1976/77. Freiburg 1978, Archivalien der FAL.

I76 Forschungszentrum FAL, Tätigkeitsbericht 1978, Freiburg im April 1979, Archivalien der FAL. 


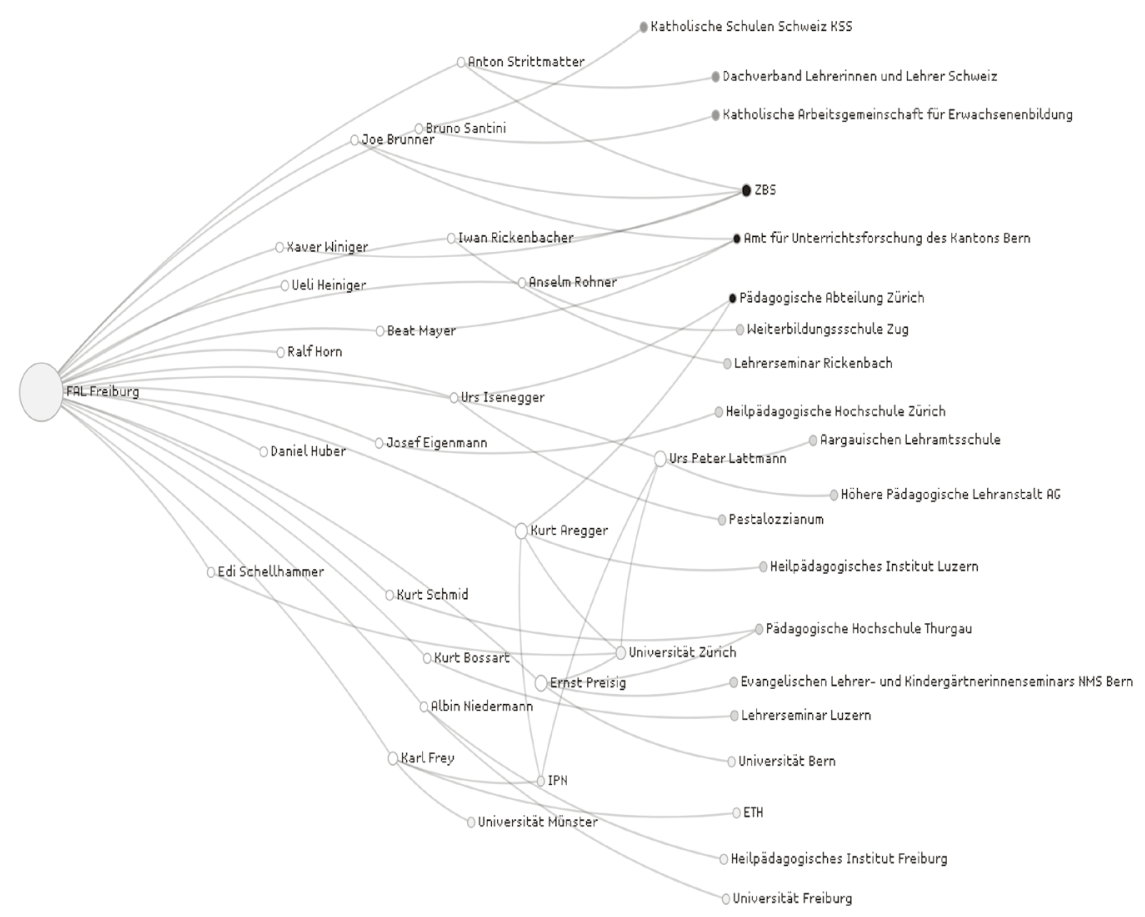

Grafik 3: Spätere Arbeitgeber der FAL-Mitglieder (eigene Darstellung)

Die Gemeinschaft der Curriculumforschung hat sich in der Wissenschaft nicht langfristig etabliert, die bei der FAL Forschenden haben ausserhalb der Wissenschaft neue Betätigungsfelder gesucht und gefunden. Der Curriculumforschung ist der wissenschaftliche Nachwuchs abhanden gekommen. Die Forschenden waren mit ihren Qualifikationen auf dem Stellenmarkt ausserhalb der Universitäten sehr begehrt.

\subsubsection{Bildungspolitisches Desinteresse und uneingelöste Versprechen}

Ein dritter Einordnungs- und Erklärungsansatz für das vorläufige Ende der Grundlagenforschung zum Curriculum in der Schweiz hängt zusammen mit dem nachlassenden Interesse in bildungspolitischen Kreisen an den Resultaten dieser Forschungsrichtung. Seit Herbst 1969 begleiteten und gestalteten einige Mitarbeiter der FAL im Rahmen des EBAC-PS-Projektes die Prozesse der damals laufenden Lehrplanreformen im deutschsprachigen Teil des Kan- 
tons Freiburg, zunächst für die Primarschullehrpläne, später auf der Orientierungsstufe. Etwas zugespitzt formuliert war das die praktische Versuchsanlage derjenigen Konzepte, welche aus der Theoriearbeit hervorgingen. ${ }^{177}$ Auch in anderen Kantonen machte sich, wenngleich etwas später, das Lehrplanreformfieber bemerkbar, die Bezüge zur Vorarbeit in Freiburg sind etwa anhand der Orientierung an Lernzielen oder Leitideen als Abbild und Ziel bildungspolitischen und pädagogischen Handelns unübersehbar. ${ }^{178}$ Aus bildungspolitischer Sicht war Grundlagenforschung in diesem Bereich nun keine Notwendigkeit mehr. Für die kantonalen Reformprojekte im Lehrplanbereich war Grundlagenforschung obsolet geworden. Aus dieser Perspektive betrachtet brauchte die Curriculumforschung auch in keinem nationalen Forschungsprogramm mehr berücksichtigt zu werden. Es sei denn, man hätte den Anspruch verfolgt, einige ungelöste theoretische Probleme ausräumen zu wollen. Diese stehen im Folgenden im Fokus.

Mit Doris Knab gesprochen, einer wissenschaftlichen Mitarbeiterin bei Saul B. Robinsohn am Max-Plack-Institut für Bildungsforschung, bestand das Ziel der Curriculumforschung in der Entwicklung eines wissenschaftlichen Instrumentariums zur systematischen Neubestimmung der Aufgaben der Schulen. ${ }^{179}$ Mit der Zeit wurden die Ansprüche aber reduziert auf die «Aufgabe, die Auseinandersetzung, die rationale Diskussion um die allgemeinen Ziele der Leitideen der Primarstufe bei allen, die sich mit Schule und Unterricht befassen, neu zu beleben». ${ }^{180}$ Nicht mehr eine theoriegeleitete wissenschaftliche Bestimmung der Aufgaben der Schule, sondern eine rationale Diskussion wurde angestrebt. Die FAL scheint sich von ihrem einstigen Anspruch auf Wissenschaftlichkeit teilweise verabschiedet zu haben, präzisierte sie doch weiter: «Leitideen sind letztlich wissenschaftlich nicht begründbar. Es sind Setzungen und damit Wertaussagen, welche in einem gesellschaftlichen und geschichtlichen Zusammenhang gesehen werden müssen.» ${ }^{181}$ Selbst an Treffen von Bildungspolitikern, Bildungswissenschaftlern und Akteuren aus Bildungsverwaltung und Schulpraxis, wie die OECD-CERI-Seminare sie organisierte, wurde in den frühen I980er-Jahren deutlich, dass die Curriculumforschung keine wissenschaftlich fundierte Antwort auf ihre einst wichtigste Frage geben konnte, nämlich warum ein bestimmtes schulisches Ziel gesetzt werden sollte. ${ }^{1{ }^{82}}$

177 Lattmann, 2015.

I78 Innerschweizer Erziehungsdirektorenkonferenz IEDK, I984, S. 2.

I79 Knab, I969.

I 80 Forschungszentrum FAL, Tätigkeitsbericht 1977, Archivalien der FAL, S. I 8.

I8 I Ebd.

I 82 Oswald, 1984 . 
Parallel dazu taten sich Probleme der Wissenschaftlichkeit auch im Bereich der Formulierung von Lernzielen auf. Unter dem Begriff Deduktionsproblem wies bereits 197I Hilbert Meyer auf den fehlenden Ableitungszusammenhang hin, der in der theoretischen Diskussion ignoriert und in den empirischen Projekten ausgeklammert werde. ${ }^{183} \mathrm{Er}$ meinte, dass die abstrakten Grobziele nicht zwingend zu den untergeordneten Richtzielen führen und daraus die Feinziele folgen würden. Jahrzehnte später sollte dasselbe Problem in den Diskussionen um Bildungsstandards wieder auftauchen, dann unter dem Begriff Taxonomieproblem. ${ }^{184}$

Die von den Mitarbeitern der FAL gemeinsam erarbeiteten Theorien und Konzepte stellten in mehrfacher Hinsicht einen Wendepunkt in der Bestimmung schulischer Inhalte dar, welche über den Anspruch einer thematischen Neuerung hinausgingen, sich als solche in der wissenschaftlichen Community aber nicht langfristig etablieren konnten. Aus wissenschaftssoziologischer Perspektive waren wohl zwei Faktoren besonders ausschlaggebend: Einerseits war die mit dem Forschungsgegenstand verbundene Normativitätsproblematik nicht aufzulösen, andererseits blieb FAL-Mitgliedern eine weitere Bearbeitung des Forschungsbereichs infolge der Nichtberufung auf Lehrstühle verwehrt.

Der Nationalfonds schrieb im Rückblick auf sein 25-jähriges Bestehen 1977: «Die Entwicklung neuer Forschungsrichtungen birgt Risiken. Der Nationalfonds nimmt in Kauf, dass nicht alle Versuche auf Anhieb zu Lösungen führen.» ${ }^{18}$ Mit anderen Worten: Grundlagenforschung muss scheitern können. Dafür liegt mit der knapp zehnjährigen Phase der Curriculumforschung ein Beispiel vor.

I83 Meyer, I97I.

I84 Criblez et al., 2009.

I85 Schweizerischer Nationalfonds zur Förderung der wissenschaftlichen Forschung, I977, S. 30 . 


\title{
3 Vom wissenschaftlichen Programm zur politischen Debatte
}

\begin{abstract}
Wir haben - wieder einmal auch im Bereich der Bildungsreform - entdeckt, wieviel weniger wir können als wir wollen. Wir haben gelernt, dass alles so viel schwieriger ist als wir dachten und dabei hatten wir es uns doch schwierig genug vorgestellt. ${ }^{1}$
\end{abstract}

Während knapp zehn Jahren waren die Mitglieder der Freiburger Arbeitsgruppe für Lehrplanforschung (FAL) intensiv im Bereich der Curriculumforschung aktiv. Parallel dazu wurde ihr Forschungsgegenstand ab den frühen I970erJahren zum Gegenstand von teilweise langwierigen und zähen bildungspolitischen Debatten im Zuge von Reformbemühungen in den Kantonen sowie auf interkantonaler und internationaler Ebene.

Dieses Kapitel beleuchtet den Verlauf dieser Debatten und fragt übergeordnet, wie und weshalb der Gegenstand der Curriculumforschung überhaupt Einzug in die bildungspolitischen Debatten in der Schweiz der 1970er-Jahre gefunden hatte. So werden die Gremien, Reformvorhaben und Projekte genannt und analysiert, die zu Treffpunkten der Verhandlung curricularen Wissens wurden. Nicht minder bedeutsam sind die Motive und Strategien der Curriculumforschenden selbst, die es aufzudecken und nach ihrer Bedeutung für Bildungsreformen zu befragen gilt.

Abschliessend wird aufgrund einer fundierten historischen Netzwerkanalyse visualisiert und diskutiert, auf welchen Wegen und unter Berücksichtigung welcher Mechanismen und kontextuellen Faktoren curriculumspezifische Forschungsergebnisse in bildungspolitischen Kontexten rezipiert wurden, ohne die involvierten Akteure und ihre strategischen Vorgehensweisen aussen vor zu lassen. 


\subsection{Kontexte der Curriculumdebatten in den 1970er-Jahren}

Bildungspolitik ist heute wie nie zuvor zu einem nationalen Anliegen geworden. Die Probleme der Schulkoordination und Schulreform haben Prioritätscharakter angenommen. ${ }^{2}$

Die Mitglieder der EDK verorteten am Ende der I980er-Jahre die dringendsten bildungspolitischen Herausforderungen der Schweiz im Bereich der Volksschule, wie sie einleitend im Jahresbericht von 1968/69 festhielten. Die EDK sei vor eine «schwerwiegende Aufgabe» gestellt, weshalb «auf bildungspolitischem Gebiet mannigfache Anstrengungen unternommen werden [...], wenn wir zu einer schweizerischen Bildungspolitik gelangen wollen». ${ }^{3}$ Der Vielfalt im Bereich der Volksschule sei mit Schulkoordination zu begegnen, so der damalige Tenor. Zu diesem Zweck rückten die Lehrpläne der Volksschule schon bald als bildungspolitisches Instrument in den Fokus nicht nur der Politik, sondern auch der Wissenschaft.

Noch bevor aber auf die politisch-programmatischen Verhandlungen von Konzepten und Reformverheissungen eingegangen wird, werden drei bedeutende Kontexte für die hierzu geführten Debatten aufgespannt. Im Bereich der öffentlichen Schule zeichnete sich erstens ein Wandel in der Strategie der gegenseitigen Angleichung der kantonalen Schulsysteme ab, wobei der Lehrplan zum Kernelement dieses Prozesses wurde. Zweitens waren die dazu entworfenen bildungs- und gesellschaftspolitischen Programme geprägt von Fortschrittsorientierung und Planungsgläubigkeit. Dies wiederum förderte drittens den Bedarf an Expertise und damit die Nachfrage nach Forschung mit unmittelbarem Verwendungszweck.

\subsubsection{Von der «äusseren» zur «inneren» Schulkoordination}

«Die Kantone befürworten von sich aus eine Reform und Koordination. Seit längerer Zeit sind solche Bestrebungen im Gange und mehrere Kommissionen bemühen sich um eine Neugestaltung unseres Bildungswesens.» ${ }^{4}$ Der Autor dieser Zeilen formulierte seine Einschätzung zur Neugestaltung des Schweizer Bildungswesens anlässlich einer ersten Publikation und Gesamtschau zur Schweizer Lehrplanvielfalt 1968.5 $\mathrm{Zu}$ diesem Zeitpunkt waren laut dem Zitat bereits einige Anstrengungen unternommen worden. Diese Bemühungen könnten durch-

2 Konferenz der kantonalen Erziehungsdirektoren, I970, S. 2.

3 Ebd.

4 Vernetz, I968, S. 560.

5 K. Frey, 1968b. 
aus als Vorboten der ein Jahr später eingereichten Volksinitiative der Bauern-, Gewerbe- und Bürgerpartei eingeordnet werden. Die Initianten verlangten eine Überarbeitung des Bildungsartikels 27 in der Bundesverfassung, legitimiert durch den Anspruch auf Chancengleichheit im Bildungswesen. Infolgedessen strebten sie eine Harmonisierung der kantonalen Schulsysteme auf Bundesebene an. ${ }^{6}$

Die Initiative bewog die EDK zu einer Reaktion auf dem Konkordatsweg: Festgelegt wurden das Schuleintrittsalter auf das vollendete sechste Altersjahr, die Dauer der Schulpflicht von neun Jahren, die Schuldauer bis zur Matura auf zwölf bis dreizehn Jahre und der Schuljahresbeginn im Herbst. Während das Schulkonkordat 197I in Kraft trat, verwarfen Bundesrat und Parlament die Initiative und legten den Stimmbürgerinnen und Stimmbürgern 1973 einen eigenen Vorschlag zur Abstimmung vor, welcher dem Bund mehr Befugnisse zur Unterstützung des Harmonisierungsvorhabens über das Konkordat hinaus einräumen wollte. Die Abstimmung scheiterte knapp am Ständemehr.?

Wie an den Zielsetzungen des Konkordats oben gut zu erkennen ist, wurde bis $\mathrm{zu}$ diesem Moment unter dem Begriff der «äusseren» Schulkoordination eine Harmonisierung schulischer Strukturen angestrebt. ${ }^{8}$ Einzelne Kantone hielten sich in den Folgejahren aber nicht an die Konkordatsbestimmungen, was die EDK zu einer Änderung ihrer Koordinationsstrategie veranlasste. ${ }^{9}$ Vermehrt sollten die schulischen Inhalte, also Lehrpläne und Lehrinhalte, zur Koordination des Schulwesens dienen. Für eine «innere» Koordination der schulischen Inhalte stützten sich die Erziehungsdirektoren auf die im Konkordat von 1970 notierten Empfehlungen in Absatz 3: ${ }^{\circ}$

«Die Konkordatskantone arbeiten zuhanden aller Kantone Empfehlungen aus, insbesondere für folgende Bereiche:

a) Rahmenlehrpläne

b) gemeinsame Lehrmittel;

c) Sicherstellung des freien Übertritts zwischen gleichwertigen Schulen;

d) Übertritt in die aufgegliederten Oberstufen;

e) Anerkennung von Examensabschlüssen und Diplomen, die in gleichwertigen Ausbildungsgängen erworben wurden;

f) einheitliche Bezeichnung der gleichen Schulstufen und gleichen Schultypen;

g) gleichwertige Lehrerausbildung.

6 Manz, 20II.

7 Criblez, 2007 a.

8 Aregger, 1971b, S. I 824; Trier, I976, S. 80.

9 Die Kantone Bern und Zürich sind bedingt durch Volksabstimmungen der Verpflichtung zur Verlegung des Schulbeginns in den Herbst nicht nachgekommen. Die Kantone Aargau, Bern, Basel-Stadt, Thurgau und Tessin sind dem Konkordat nicht beigetreten.

I0 «Konkordat über die Schulkoordination», 29. I0. 1970, www.edk.ch/dyn/ I 43 I I.php, abgerufen am 22. 3. 2020. 
Die Konferenz schweizerischer Lehrerorganisationen ist bei der Ausarbeitung dieser Empfehlungen anzuhören.»

Insbesondere die Empfehlungen zur gemeinsamen Ausarbeitung von Rahmenlehrplänen und Lehrmitteln sind für den vorliegenden Zusammenhang bedeutsam. Die Innerschweizer Erziehungsdirektorenkonferenz ${ }^{\text {II }}$ (IEDK) hat alsbald die Beschäftigung mit den Empfehlungen aufgenommen und 1972/73 die Erarbeitung eines gemeinsamen Lehrplanes für die Sekundarschulen in die Wege geleitet. ${ }^{12}$ Seit auf interkantonaler Ebene die Einführung von neun obligatorischen Schuljahren propagiert wurde, waren etliche Kantone mit der Aufgabe konfrontiert, ein bis zwei neue obligatorische Schuljahre einzuführen und die schulischen Inhalte dafür zu bestimmen. ${ }^{13}$ Anlässlich dieses neuen, «inneren» Koordinationsprozesses sind sich die Erziehungsdirektoren bewusst gewesen, «dass diese Reformprojekte noch weit stärker an die bestehenden Strukturen rühren würden». ${ }^{14}$ Die Auseinandersetzung mit den schulischen Strukturen war nicht abgeschlossen, neu rückten aber die Fragen nach Auswahl, Aufbereitung und Fixierung schulischer Inhalte in Lehrplänen ${ }^{15}$ in den Vordergrund.

\subsubsection{Fortschrittsorientierung und Planungsgläubigkeit}

Jener Wandel der Koordinationsbestrebungen der Kantone hin zu Reformen über die schulischen Inhalte vollzog sich mindestens zu Beginn vor dem Hintergrund einer bereits in den I960er-Jahren konstatierten gesellschaftlichen Tendenz, welche als «Planungsgläubigkeit» und «Fortschrittsorientierung» charakterisiert wurde. ${ }^{16}$ Unter dem Zwischentitel «Entdeckung politischer Planung im Bildungssystem» beschreiben Kussau und Oertel, ${ }^{17}$ dass die angestrebte Veränderung und der Ausbau des Bildungswesens in der Schweiz im Zuge der Bildungsexpansion ${ }^{18}$ Effekte auf die Aufgaben und Tätigkeiten der kantonalen Erziehungs- und Bildungsdirektionen hatten. Allerdings ist darauf hinzuweisen, dass es sich beim Planen von Projekten und Vorhaben keines-

I I Zu diesem Verbund gehören die Kantone Luzern, Uri, Schwyz, Obwalden, Nidwalden, Zug und der deutschsprachige Teil des Kantons Wallis. Jahresbericht der Konferenz der kantonalen Erziehungsdirektoren 1972/73. Bern: EDK.

I3 Rickenbacher, 1972b, S. 27.

I4 Egger und Blanc, 1977, S. 3 .

Is Begriffsgeschichtlich interessant ist die Feststellung, dass die EDK in ihren Berichten und Protokollen den Begriff «Lehrplan» nie durch den Begriff «Curriculum» ersetzte, wie das etwa die Fachzeitschriften zu tun pflegten.

I6 Honegger, 2007; Stettler, I994.

I7 Kussau und Oertel, 200I, S. I 44.

I 8 Zur Erklärung des Begriffs siehe Kapitel I. 
falls um ein rein schweizerisches oder bloss bildungsspezifisches Phänomen handelte: In nahezu allen Politikbereichen hat «Planung» als Instrument der Umgestaltung international die gesamtgesellschaftlichen Umbruchsituationen seit den frühen I960er-Jahren geprägt. ${ }^{19}$

Eine lange Phase des Wirtschaftswachstums, der wissenschaftliche und technologische Wandel sowie wohlfahrtsstaatliche Neuerungen haben «eine Grundstimmung der Machbarkeit und Planbarkeit» gefördert. ${ }^{20}$ Mit den wirtschaftlichen Einbrüchen in den I970er-Jahren gingen zwar «Ernüchterung, Nachlassen des Reformelans, ja Anzeichen von Planungsmüdigkeit und Reformverdrossenheit und in der Bildungsdiskussion regressiv-konservative Haltungen ${ }^{21}$ einher, sie taten aber den Diskussionen im Bereich der Lehrplanreformen keinen Abbruch. Im Herbst 1976 widmete die pädagogische Kommission der $\mathrm{EDK}^{22}$ dem Gegenstandsbereich der Schulkoordination über Lehrplanreform ein ganztägiges Seminar mit dem Ziel, einen Überblick im Bereich der Lehrpläne der Volksschule zu gewinnen und die Kantone gleichsam zur engeren Zusammenarbeit in diesem Bereich aufzurufen. ${ }^{23}$ So wenig die Bildungspolitik vom Themenbereich der Schulreform über die Inhalte abrückte, so langjährig gestalteten sich die eingeräumten Lehrplanrevisionen. Bis in die frühen I990er-Jahre und damit bis zur Verschiebung des schulpolitischen Interesses hin zu Fragen der Schulqualität und -wirksamkeit blieben die Lehrpläne bedeutender Reformgegenstand in den Kantonen. ${ }^{24}$

Anders als von Saul B. Robinsohn am Max-Plack-Institut in Berlin einst angedacht, ${ }^{25}$ waren die ersten Reformen der Curricula der 1970er-Jahre in der Schweiz partizipativ angelegt, Lehrpersonen wurden in die Verfahren mit einbezogen. ${ }^{26}$ Beobachter der Debatten meinten in dieser Form der Mitsprache und Mitbestimmung gar eine «Ideologisierung der Diskussion» ${ }^{27} \mathrm{zu}$ spüren und Rudolf Künzli ${ }^{28}$ schrieb rückblickend, die Verantwortlichen hätten sich in

I9 Bosche, 20I3; Bürgi, 2017.

20 Criblez, 2001 a, S. 98.

2 I Widmer, I976, S. Is f.

22 Gegründet wurde die pädagogische Kommission (PK) im Juli 1967 mit dem Ziel, die EDK in allen Belangen hinsichtlich der anzustrebenden Koordination des Schweizer Schulwesens zu beraten.

23 Jenzer et al., 1978.

24 Künzli, Fries, Hürlimann und Rosenmund, 2013.

25 Robinsohn, 1967.

26 Eine genauere Beschreibung der Partizipationsformen von Lehrpersonen in Lehrplanreformen ist in Kapitel vier zu lesen.

27 Widmer führt die partizipativen Organisationsweisen der Verfahren auf Studentenunruhen und politische Forderungen im Zuge der Demonstrationen von i 968 zurück. Widmer, I976, S. I I.

28 Rudolf Künzli hatte am Institut für Erziehungswissenschaft in Zürich I999-2006 eine Titularprofessur inne. Er doktorierte 1970 an der Universität Zürich, war dann bis 1988 am 
ihre Debatte um die Festlegung der in den Arbeitsgruppen notwendigen «Verfahren und Modi» verbissen. ${ }^{29}$ Das Planen war zur Selbstverständlichkeit und in Anbetracht der mehrjährigen Verfahren und der Vielzahl zu involvierender Akteure zur Notwendigkeit geworden, auch wenn sich das eigentliche Ziel, die Harmonisierung kantonaler Lehrpläne, vorerst nicht realisieren lassen sollte. ${ }^{30}$ Dennoch herrschte bis in die späten I980er-Jahre in Wissenschaft, Politik und Verwaltung die Überzeugung vor, dass die Gestaltung des Schulsystems nur mittels der richtigen Informationen der neuen Komplexität gerecht werden könne. ${ }^{3 \mathrm{I}}$ «Bildungspolitik als Gesellschaftspolitik muss strategisch orientiert sein und durch sachliche Informationen vorbereitet werden», ${ }^{32}$ war demgemäss zu vernehmen. Der Bedarf an Information zur sachgerechten Vorbereitung von politischen Entscheidungen war verbunden mit der Hoffnung auf eine Effektivierung politischen Handelns, was der Verbreitung eines bestimmten Forschungstypus, der sogenannten Verwendungsforschung, Vorschub leistete. ${ }^{33}$

\subsubsection{Verwendungsforschung und Bedarf an Expertise}

Forschende im Bereich der Verwendungsforschung hatten es sich zur Aufgabe gemacht, «das Vertrauen in die sozialen und technologischen Problemlösungspotentiale professioneller Berater» zu nutzen, und beabsichtigten gemäss Strassheim den Transfer von wissenschaftlichem Wissen in die Praxis voranzutreiben. ${ }^{34}$ Die für den vorliegenden Zusammenhang aktiven Forschenden mit Ambitionen auf eine Verwendung ihrer Resultate für gesellschaftliche Problemstellungen stammten vornehmlich aus den Sozialwissenschaften. ${ }^{35}$ Beck und Bonss beschrieben anlässlich des 22. Deutschen Soziologentages das Ziel verwendungsorientierter Forschung. Dieser Bereich beschäftige sich primär mit

Institut für Pädagogik der Naturwissenschaften (IPN) in Kiel (D) tätig und habilitierte sich 1983 an der Christian-Albrechts-Universität zu Kiel. Er war Leiter der aargauischen Bezirkslehrerausbildung, Direktor der Pädagogischen Hochschule an der Fachhochschule Aargau und Gründungsdirektor der Pädagogischen Hochschule der Fachhochschule Nordwestschweiz. In seinen wissenschaftlichen Arbeiten beschäftigte er sich umfassend und regelmässig mit dem Lehrplan; als Gestaltungsinstrument, als Schulprogramm (2013) oder als Auftrag der Gesellschaft (201 I).

Künzli, 2006b, S. 88.

30 Künzli, I999; 2006a, S. 64.

3 I Kussau und Oertel, 200 I.

32 Widmaier, 1968, S. 52.

33 Wingens, 1988.

34 Strassheim, 2014, S. 68.

35 Beck und Bonss, 1985; König und Zedler, 1998. Zu Funktion und Bedeutung naturwissenschaftlicher Expertise in Beratungs- und Steuerungsgremien vgl. beispielsweise Jasanoff, 1994; Vandendriessche, 2015. 
Praxisstrukturen. «Ihr Gegenstand sind real existierende Verwendungsfelder, professioneller wie nicht-professioneller, organisierter wie nicht-organisierter, öffentlicher und privater Art.» ${ }^{36}$ In diese weit gefasste Beschreibung potenzieller Betätigungsfelder von Verwendungsforschung fällt zweifelsohne auch das Bildungswesen.

Wingens verortet die Anfänge der verwendungsorientierten sozialwissenschaftlichen Wissensproduktion in den USA nach 1945. In soziologischen Arbeiten wurde versucht, die Bedeutung der Ergebnisse für die politisch-praktische Arbeit herauszuheben. In der Bundesrepublik Deutschland haben sich erst in den I960er-Jahren beiläufige Kontakte zwischen Sozialwissenschaft und Politik ergeben, wobei die Diskussionen eher im Abstrakten, Übergeordneten verlaufen sind. Es bedurfte der allgemeinen Einsicht, dass gesellschaftspolitische Planung eine Notwendigkeit darstelle, bis politische Akteure Erkenntnisse der Verwendungsforschung als Hilfsmittel politischer Planung in den Blick nahmen. ${ }^{37}$ Stand für Verwendungsforschende zunächst die Bereitstellung von Wissen zur rationalen Planung im Vordergrund, sollte dieses Wissen bald auch «eine Veränderung in der Problemsicht der Politiker bewirken und im Ausloten von Handlungsspielräumen mögliche alternative politische Strategien aufzeigen».$^{38}$ Die Politik, das Verwaltungswesen und die Wissenschaft beabsichtigten, gemeinsam gesellschaftliche Handlungsfelder zu gestalten. Das heisst aber auch, dass Forschung über ihre angestammte Aufgabe des Analysierens hinaus normative Entscheidungen zu fällen und mitzutragen hatte und somit politisch wurde. Allerdings ist dieses auf einem Rationalitätskontinuum basierende Konzept zu Beginn der I980er-Jahre widerlegt worden. ${ }^{39}$ Als Begründung führt Drerup an: «Die Wege wissenschaftlicher Informationen in der Wissenschaft, von der Wissenschaft in ausserwissenschaftliche Bereiche sind vielfältiger und unübersichtlicher als es die konventionellen Fahrpläne «naiver〉 Verwendungsforschung vorsehen.»40

Die bisherigen Ausführungen verdeutlichen, dass der Einbezug rationaler Wissensbestände in politische Zusammenhänge zur Lösung gesellschaftlicher Probleme ein zweiseitiger war: Planungsrationalitäten und komplexe Problemstellungen förderten einerseits das Bedürfnis nach wissenschaftlichen Einschätzungen zuhanden von Politik und Verwaltung. ${ }^{41}$ Andererseits entdeckten Forschende in praxisgebundenen Problemstellungen einen neuen, attraktiven

37 Wingens, I988.

38 Ebd., S. 56.

39 Drerup, I989.

40 Ebd., S. I 6.

4 I Criblez, 20i6a. 
Tätigkeitsbereich, welcher eine gute Auftragslage und die Bearbeitung neuer Fragestellungen in Aussicht stellte..$^{42}$ Die produzierten Forschungsresultate wurden zwar politischen und nicht wissenschaftlichen Rationalitäten gemäss rezipiert. ${ }^{43}$ Wie im weiteren Verlauf dieses Kapitels gezeigt wird, hatten die Forschenden aufgrund der Vermittlung und Erläuterung ihrer Resultate einigermassen grosse Gestaltungsspielräume, wurden sie doch in unterschiedliche Gremien als Experten mit einbezogen.

«Experience alone is not an indicator of expertise», schreiben Bradley, Paul und Seeman in ihrem Beitrag zur Struktur von Expertenwissen. ${ }^{44}$ Zwar sei das Vorhandensein von spezialisiertem Wissen eine notwendige, aber noch keine hinreichende Bedingung, um als Expertin oder als Experte zu gelten. In der einschlägigen Literatur ist man sich einig, dass kein fixer Kriterienkata$\log$ zur Definition eines Expertenstatus vorliegt. ${ }^{45}$ Schützeichel wie Tenorth meinen etwa, das Expertentum sei eine soziale Relation und könne nicht nur wissenssoziologisch gefasst werden. Jemand könne nur Experte sein, wenn er als solcher anerkannt werde. ${ }^{6}$ Carrier wiederum unterscheidet zwischen drei verschiedenen Formen von Expertentum: ${ }^{47}$ Er nennt als Erstes die technischen Experten, welche sich an vorgegebenen Regelwerken orientieren und diese anwenden; zweitens die professionellen Experten, welche wie Ärzte oder Richter Einzelfälle unter Berücksichtigung von Präzedenzfällen beurteilen. Die dritte Form von Expertentum sei eine wissenschaftliche. Hierbei würden Probleme behandelt, deren Lösung wiederum in wissenschaftliches Wissen eingepasst werde oder dieses ergänze. In Bezug auf die Funktion von wissenschaftlichen Experten in ausserwissenschaftlichen Feldern wie der Politik weist Lengwiler schliesslich darauf hin, ${ }^{4}$ dass sie auch als Grenzgänger zwischen zwei Welten oder als Übersetzer eingeordnet werden müssen. ${ }^{49}$

Sozialwissenschaftliche Verwendungsforschung steht auch als Überbegriff für Formen der «Begleitforschung», wie die in den I970er-Jahren zunehmend betriebene praxisorientierte Bildungsforschung auch bezeichnet wurde. Dazu weiterführend Weishaupt, I99I. Criblez, 2008, S. I 59.

44 Bradley, Paul und Seeman, 2006, S. 77.

45 Amann, 1994; Brosziewski, 2016; Trischler, 201 5.

46 Schützeichel, 2007a; Tenorth, 20I4b.

47 Carrier, 20I4.

48 Lengwiler, 2010.

49

Wie vor dem Hintergrund von «technokratischen Kausalitätsschemata» ganze Organisationen zu international wirkmächtigen Bildungsexpertinnen werden können, hat Regula Bürgi am Beispiel der OECD herausgearbeitet. Bürgi, 2017, S. 2 I 2 f. 


\subsection{Programmatik der Curriculumforschung}

Im vorherigen zweiten Buchteil zu Aufstieg und Niedergang der Curriculumforschung in der Schweiz ist offensichtlich geworden, dass die Mitglieder der Freiburger Arbeitsgruppe für Lehrplanforschung (FAL) fast ausschliesslich die Deutungshoheit über den Begriff Curriculum im wissenschaftlichen Feld in der Schweiz innehatten. Massgeblich dazu beigetragen hat ihre vergleichsweise imposante Publikationstätigkeit, die nicht nur an etlichen Monografien und Sammelbänden erkennbar ist, sondern auch an der Anzahl Artikel in einschlägigen Fachzeitschriften der Wissenschaft oder in Lehrerzeitungen abzulesen ist.

In diesem dritten Teilkapitel geht es erstens um die politischen Ansichten, Vorstellungen und Legitimationen der Forscher aus Freiburg in Bezug auf Bildungsreformen. Insbesondere in den Artikeln für Lehrerzeitschriften und in Buchbeiträgen haben die Mitglieder der FAL festgehalten, weshalb Curriculumreformen in den Kantonen dringlich sind und wie diese anzugehen sind. Damit legten sie gleichsam ihre Ansichten darüber offen, inwiefern Wissenschaftler in die anzugehenden Reformen mit einzubeziehen sind.

Zweitens werden anhand von Quellenmaterialien aus den Archivalien der FAL Strategien und Massnahmen präsentiert, anhand deren sich die Mitglieder der FAL Gehör und Sichtbarkeit in einer breiteren Öffentlichkeit zu verschaffen beabsichtigten. Dazu werden insbesondere ihre Konzepte der Öffentlichkeitsarbeit und ihre Thesen zu Bildungsplanung und Schulreform diskutiert.

\subsubsection{Legitimationen von Curriculumreformen}

Der Gründer und Leiter der Freiburger Arbeitsgruppe Karl Frey publizierte I 969 in der Zeitschrift «Die Deutsche Schule» einen Artikel mit dem Titel «Das Curriculum im Rahmen der Bildungsplanung und Unterrichtsvorbereitung»..$^{\circ}$ Freys Beitrag liess sich durchaus anschliessen an eine Debatte, die in Deutschland unter Mitwirkung der Organisation für wirtschaftliche Zusammenarbeit und Entwicklung (OECD) bereits seit ein paar Jahren Fahrt aufgenommen hatte. So veranstaltete das Institut für Bildungsforschung in der Max-PlanckGesellschaft in Berlin 1967 ein mehrtägiges internationales Seminar zum Thema Bildungsplanung und kam nicht umhin, im Tagungsbericht auf die wiederholt konstatierten Lücken in der Curriculumforschung als Instrument der Bildungsplanung hinzuweisen. ${ }^{5 I}$ Ein Jahr später dann verwies Heinrich Roth

5 I Edding, Jensen und Ulshoefer, I967. 
auf die «kommende Revolution der Inhalte» im Bildungswesen, um nichts weniger als unter Zuhilfenahme der Wissenschaft «das Gemeinwohl der Gesellschaft $\mathrm{zu}$ sichern».52

Im Artikel von Karl Frey wird der Hintergrund dieser Rhetorik deutlich. In seinen Ausführungen setzt er voraus, dass «Bildungs- und Wirtschaftswachstum voneinander abhängig sind und wenn die Bildungseffizienz mit der Prosperität der Gesellschaft nach der Art eines Regelkreises im Zusammenhang steht, dann müssen sich die Unterrichtsleistungen [...] mit den gesellschaftspolitischen Zielen decken». ${ }^{33}$ Das nimmt Frey zum Anlass, um das Curriculum als zentrales Planungs- und Gestaltungselement von Unterricht zu propagieren. Um den Anforderungen der Planung als systematischer Abgleich des Bildungswesens mit wirtschaftlichen und gesellschaftlichen Ansprüchen zu genügen, müsse ein Curriculum als Dokument Aussagen zu vier Dimensionen machen: «(I) Funktionen: Bildungsziele, Lernziele, (2) Inhalte: Lerngegenstände, Unterrichtsthemen, Fächer, (3) Inhaltstruktur: didaktische Struktur, formalisierte Inhalte in Hinsicht auf die Lernziele, (4) Unterrichtsorganisation: Lernverfahren, Hilfsmittel.» ${ }^{4}$

Ein Curriculum musste nach Frey dem Anspruch nachkommen, gesellschaftspolitische Ziele in einen zirkulären und stetigen Abgleich mit Unterricht zu bringen, weshalb die Formulierung und Überprüfung von Lernzielen in den I970er-Jahren zum Kernelement der Curriculumforschung avancierte.5" In der katholischen Lehrerzeitung "Schweizer Schule» warb Bruno Santini ebenfalls für «die Bedeutung des Curriculums in der inneren und äusseren Bildungsplanung». ${ }^{6}$ Auch er erläuterte zunächst, dass ein Curriculum für die Bildungsplanung zweierlei Funktion habe: Eine erste, innere Planung bestehe in der Unterrichtsvorbereitung von Lehrpersonen, die von noch festzulegenden Bildungszielen herzuleiten sei. Für die zweite, äussere Planung erfülle das Curriculum eine Schlüsselfunktion der Koordination im Schulwesen. Erst wenn die Curricula die Minimalanforderungen und Abschlussziele eines Schultyps oder einer Schulstufe festlegen würden, könnten die bestehenden Probleme der Durchlässigkeit zwischen zwei Schultypen innerhalb einer Ortschaft, aber auch zwischen verschiedenen Ortschaften oder Kantonen gelöst werden.

52 Roth, I968, S. 69.

53 K. Frey, 1969a, S. 274.

54 Ebd., S. $270 \mathrm{f}$.

55 Dass es sich bei diesem zirkulären Konzept nicht um eine Neuerung handelte, sondern bereits etwa Ralph W. Tyler in seiner Publikation «Basic Principles of Curriculum and Instruction» (1949) die stetige Evaluation eines Curriculums vorgeschlagen hatte, hat Göldi (20I I) herausgearbeitet.

56 Santini, 1970. 
Santini äusserte sich ferner zu den aktuellen Lehrplänen und sparte nicht mit Kritik. In Bezug auf eigens durchgeführte Befragungen bei Lehrkräften und abgestützt auf die Lehrplanuntersuchungen der FAL wies er auf die derzeit knappen Darstellungen von «stichwortartigen Stoffprogrammen» hin. ${ }^{57} \mathrm{Er}$ konkretisierte: «Diese Ausführungen sind meist so kurz und allgemein gehalten, dass sie zwar für die langfristige Unterrichtsplanung, d. h. für ein Semester oder ein Jahr, dienen können, nicht aber für die wöchentliche oder gar tägliche Unterrichtsvorbereitung.» ${ }^{8}$

Iwan Rickenbacher legitimierte die «Curriculumreform als Ansatz der Schulreform» und verwies erstens auf einen markanten Modernitätsrückstand der Schule.59 Er machte einen beschleunigten gesellschaftlichen Wandel geltend, der dazu führe, dass das bestehende Lernangebot in der Schule nicht mehr auf die künftigen Lebenswelten vorbereite. Die Bezugswissenschaften der meisten Schulfächer hätten sich weiterentwickelt und würden einen merklich grösseren Wissensstand ausweisen. Demgegenüber habe der Wissensstand der Schulbücher und auch der Lehrpersonen stagniert.

Darüber hinaus verwies Rickenbacher auf die aufstrebenden Wirtschaftswissenschaften oder die Sozialwissenschaften, welche noch keine Entsprechung in den Lehrplänen gefunden hätten. «Die Geschwindigkeit jedoch, mit der wissenschaftliche Erkenntnisse bis auf die Ebene des Schulfaches tradiert werden, könnte erhöht werden.» ${ }^{60}$ Ferner habe man es bisher verpasst, bei der Festlegung von Bildungszielen oder Stundentafeln, aber auch in der Unterrichtsplanung auf die neusten Ergebnisse der Lern- und Entwicklungspsychologie zurückzugreifen.

Mit Bezug auf einen Artikel zur Verwendung von Unterrichtstechnologien in der Schulpraxis von Karl Frey ${ }^{61}$ verdeutlichte Rickenbacher ein weiteres Argument, weshalb eine Reform des Schulwesens mit dem Curriculumansatz angezeigt wäre: Frey vertrat in seinem Beitrag für die «Zeitschrift für Lehrerbildung» die These, dass «die Lehrer und ihre Mitarbeiter in den Schulverwaltungen und Ausbildungsstätten die angebotenen technischen Instrumente pädagogisch und lernpsychologisch nicht richtig einzusetzen, das heißt Wert und Wirkung zu beurteilen vermögen». ${ }^{62}$ Die Beurteilung des Nutzens von Fernsehgeräten, Lernmaschinen, Projektoren oder Tonbandgeräten fiele den Lehrpersonen und allen anderen genannten Unterrichtsexperten bedeutend leichter,

57 Ebd., S. 526.

58 Ebd.

59 Rickenbacher, 1972a.

60 Ebd., S. 60.

6I Zu Schulversuchen mit neuen Unterrichtstechnologien und Lernmaschinen vgl. Deplazes, im Druck.

62 K. Frey, I970b, S. 1009. 
so dann die Argumentation von Rickenbacher, wenn ihr Einsatz im Unterricht mittels eindeutig formulierter Lernziele überprüft würde. ${ }^{63}$ Und dazu würden die traditionellen Lehrpläne nicht Hand reichen. Es bedurfte nach dem Verständnis der Curriculumforscher der Universität Freiburg einer Reform der bestehenden Lehrpläne nach den neusten Erkenntnissen aus ihrer Forschung.

\subsubsection{Herausforderungen der Curriculumkonstruktion}

So klar die Kritik an bestehenden Lehrplänen formuliert und die Forderung nach einer Reform der Curricula positioniert war, so disparat waren die Forschungsergebnisse zu Beginn der I970er-Jahre hinsichtlich der nun einzuleitenden Schritte unter Berücksichtigung der selbstauferlegten wissenschaftlichen Prämissen. Anschaulich repräsentiert wird dieser Moment der akademischen Unklarheit in einer von Saul B. Robinsohn verantworteten Aufsatzreihe zur Curriculumproblematik. ${ }^{64}$ Robinsohn positionierte einleitend die Curriculumarbeit als «policy science», als «politik-orientierte Wissenschaft». ${ }^{65}$ Ihre Ziele seien «Reflexion und Interpretation kultureller Traditionen, sozial- und verhaltenswissenschaftliche Teiltheorien, empirische Evidenzen und Entscheidungsmodelle so, dass praktische Operationen rationalisierbar werden. Normen, Ziele und Mittel werden in einen Begründungszusammenhang gebracht, Erkenntnisse und Erkenntnisinstrumente verschiedener wissenschaftlicher Disziplinen werden in ihre bildenden Funktionen transponiert, gegenwärtige und antizipierte Bedürfnisse konstatiert und postuliert, hypothetisch oder geprüft systematisierte Erfahrungen über Wirksamkeit von Lehren und Lernen eingebracht, und aus all diesen Interessen und Erkenntnissen werden durch systematische und offengelegte Kommunikation haltbare Alternativen ermittelt.» ${ }^{66}$ Die oben genannten Zielsetzungen aber würden das Problem mit sich bringen, dass sie weder streng wissenschaftlich fundiert seien noch der vorliegenden gesellschaftlichen und politischen Dringlichkeit nachkämen, schrieb Robinsohn weiter. Sie hätten ferner die Diskussion eröffnet über didaktische und unterrichtsorganisatorische Vorstellungen, aber auch Raum geboten für fundamentale Schulkritik und Skepsis gegenüber der Ausführbarkeit wissenschaftlicher Konzepte. Letztlich plädierte Robinsohn für den Versuch, «radi-

63 Rickenbacher, 1972a.

$64 \mathrm{Vgl}$. dazu die Ausgaben 5/1971 und 2/1972 in «Bildung und Erziehung». Die Beiträge sind später in Buchform erschienen unter dem Titel «Curriculumentwicklung in der Diskussion Robinsohn» (1972).

65 Robinsohn, I97Ib, S. 322.

66 Ebd. 
kale Curriculumreform von den Zielen her zu legitimieren», ${ }^{67}$ wohl wissend, dass die dazu erforderlichen Methoden, Möglichkeiten und Grenzen erst auszuloten seien.

Ebensolche Optionen legten Karl Frey und Urs Isenegger in Robinsohns Band von 1972 vor. ${ }^{68}$ Sie gingen davon aus, dass ein Curriculum ein Dokument sei, das konstruiert und später implementiert würde. Sie präsentierten zunächst zwei mögliche Vorgehensweisen zur Frage, womit die Konstruktion eines Curriculums beginnen sollte: Entweder schliesse die Entscheidung für ein bestimmtes Curriculum die Sammlung und Auswahl von Bildungs- und Lernzielen bereits mit ein oder der Konstruktionsprozess beginne erst, nachdem eine Fixierung der Lerninhalte erfolgt sei. Anschliessend diskutierten Frey und Isenegger beide Varianten vor dem Hintergrund der Frage, welche Kriterien für eine Auswahl der Lernziele herangezogen werden müssten, und wiesen darauf hin, dass hierfür noch keine handreichende Theorie der Curriculumkonstruktion bestehe.

Selbst die vorgeschlagene Legitimation von Kriterien der Auswahl von Lernzielen durch empirische Erhebungen im Praxisfeld etwa bei Lehrpersonen konnte das Dilemma einer theorie- oder «satzsystem»-basierten Curriculumkonstruktion nicht auflösen; ${ }^{69}$ sie schien mehr Fragen aufzuwerfen, als sie zu beantworten vermochte. Die Debatte um eine gelingende Konstruktion von Curricula wurde wenig später von einer Erweiterung geprägt: Welche Akteure sind sinnvollerweise überhaupt an einer Konstruktion von Curricula zu beteiligen?

Demgemäss fragte Ueli Heiniger in einem Beitrag für die «Schweizer Schule», $\mathrm{ob}$ «Curriculumentwicklung an der Basis» erfolgen sollte. ${ }^{\circ 0}$ nachdem er 1973 während zweier Wochen im Auftrag der Schweizer Abteilung für Wissenschaft und Forschung des Departements des Innern als Abgeordneter an einer internationalen Tagung zu diesem Thema teilgenommen hatte. Die Erfahrungen in anderen Ländern, namentlich den USA, Grossbritannien und Schweden, hätten gezeigt, dass Curriculumentwicklung nach dem Ansatz «Forschung - Entwicklung - Vermittlung ${ }^{{ }^{11}}$ bloss geringen Ertrag bringe: «Produkte, die von

67 Robinsohn, I97Ib, S. 322.

68 K. Frey und Isenegger, 1972.

69 Ebd., S. 69.

70 Heiniger, 1974 .

7I Dieser Ansatz, auf welchen in der Planung der Curriculumkonstruktion auch zurückgegriffen wurde, evozierte eine starke Trennung zwischen der theoretischen Ausarbeitung eines Curriculums und der konkreten Anwendung der entstandenen Produkte, etwa in der Schulpraxis. Daneben wurde auch über die Verwendung von Problem-SolvingModellen nachgedacht, welche eine Mitsprache bei der Problemdefinition und -ausarbeitung durch Betroffene vorsah. Eine noch offenere Vorgehensweise in der Planung und 
«oben〉 nach <unten〉 entwickelt wurden, haben sich im Klassenzimmer nicht durchgesetzt.» ${ }^{72}$ Infolgedessen schlug Heiniger vor, diesen Ansatz für die Curriculumentwicklung in der Schweiz weiter zu prüfen.

Schulzentrierte Curriculumentwicklung, Curriculumentwicklung in der Schule und schulnahe Curriculumentwicklung waren für Heiniger gleichbedeutend und bezeichneten einen dezentralisierten, partizipativen Prozess. Dieser Prozess erfordere zwar «massvolle Koordination», anders als die bisherigen Entwürfe werde er aber angebahnt, geplant und realisiert von «allen in der täglichen Schularbeit Beteiligten (Lehrer, Eltern, Schüler, Schulverwaltung)».73 Karl Frey wiederum diskutierte 1975 diese Variante der Auswahl und Bündelung von schulischen Inhalten zur Konstruktion von Curricula als Modell «diskursiver Legitimation». ${ }^{74}$ Sein Ansatz für eine Theorie der Rechtfertigung von Bildungsinhalten ist als Versuch zu verstehen, solche Diskussionen unter Teilnehmenden mit unterschiedlichem beruflich-gesellschaftlichem Hintergrund zu versachlichen. Dazu erforderlich sei ein «curricularer Bezugsrahmen», und Frey meinte damit: «Bedingungen, Vorentscheidungen, gesamtcurriculare $\mathrm{Zu}$ sammenhänge und Zielorientierungen bilden den inhaltlichen Teil des «Curricularen Bezugsrahmens für das jeweils konkret zu erarbeitende Curriculum.»75 Die Diskussionsteilnehmenden müssten sich nach Frey also, noch bevor sie sich an die konkrete Konstruktion eines Curriculums heranwagen, auf übergeordnete Absichten und Ziele des auszuarbeitenden Dokumentes einigen.

Die bisherigen Ausführungen verdeutlichen, dass die Curriculumforscher die beabsichtigte Konstruktion von Curricula in theoretischen Überlegungen von teilweise schwer nachvollziehbarer Art dargelegt hatten. Jede Entscheidung zur Auswahl von Inhalten, deren Reihung oder die Art und Weise ihrer Aufbereitung war ihren Modellen gemäss in Passung zu bringen mit einer übergeordneten Referenz, sei das ein Bezugsrahmen, ein Leitsystem oder ein Satzssystem. Konsequenterweise setzte die Festlegung solcher Satzsysteme wiederum Entscheidungsprozesse voraus, die wiederum bestimmte Setzungen erforderlich machten. Die Forschung zur Curriculumkonstruktion war getragen von der Überzeugung, die dazu nötigen Prozesse seien planbar, und übertrug sie auf Entscheidungssituationen in der Curriculumkonstruktion. Von der theoretischen Unlösbarkeit dieser Aufgabe war in den politisch-programmatischen Positionierungen der Schweizer Forscher nichts zu lesen.

Einleitung von Curriculumkonstruktionen sahen verschiedene Partizipationsmodelle vor. Weiterführend Nezel und Ghisla, 1977.

72 Heiniger, 1974 , S. 19.

73 Ebd., S. I4.

74 K. Frey, I975.

75 Ebd., S. I07. 


\subsubsection{Curriculumreform als Instrument der Schulreform}

Mitte 197I veröffentlichte die «Schweizer Schule» Is Thesen zur Bildungsplanung und Schulreform. ${ }^{76}$ Bei den Autoren der Thesen handelte es sich um die Mitglieder der Freiburger Arbeitsgruppe für Lehrplanforschung. Dieser Beitrag ist für den vorliegenden Zusammenhang aus zwei Gründen von Bedeutung. Erstens liegen in den Lehrerzeitschriften keine vergleichbaren Positionsbezüge oder Vorschläge dazu vor, welche die aktuellen bildungspolitischen Herausforderungen skizzierten und ein Programm darlegten, mit welchen Schritten nun vorgegangen werden sollte. Zweitens ist ungeachtet der inhaltlichen Positionierung der Autoren bemerkenswert, dass sich Wissenschaftler zu aktuellen Themen und Fragen von explizit bildungspolitischer Dimension überhaupt in einer Lehrerzeitschrift äusserten.

Die Is Thesen des Zeitschriftenartikels ${ }^{77}$ werden im Folgenden in vier Punkten zusammengefasst und diskutiert. Sie verstehen sich als bezeichnendes Beispiel dafür, wie Wissenschaftler, deren Forschungsgegenstand hauptsächlich von Schulreformen und Curriculumtheorie geprägt war, ihren Forschungsbereich bildungspolitisch positionierten. Die Zusammenfassung des ersten Themenbereichs lautet:

I. Schulreform ist gleichbedeutend mit Curriculumreform, also der Reform von Lehrplänen, Unterrichtsformen und der Unterrichtsorganisation. Eine solche Reform muss geplant werden und bedarf überprüfbarer Lernziele.

Hier wird die Frage beantwortet, was eine Schulreform überhaupt ist und mit welchen Instrumenten und anhand welcher Steuerungsmittel sie vollzogen werden soll. Es wurde proklamiert, dass es zur Schulreform einer Planung bedürfe und dass das Instrument der Steuerung von Schule und Unterricht der Lehrplan sei. Diesen gelte es zu reformieren und mit Lernzielen anzureichern. Unterrichtsformen und Unterrichtsorganisation sollten in Lehrplänen festgeschrieben werden.

2. Schulreform ist ein partizipativer Prozess. Lehrpersonen, politische Parteien, die Wirtschaft, Eltern und Schülerinnen und Schüler, Forschende aus der Wissenschaft und alle anderen interessierten Gesellschaftsgruppen sind an der Debatte um die Zielsetzungen zu beteiligen.

Die FAL legte in ihrem Beitrag einige Thesen vor, welche die in einer Schulreform zu involvierenden Akteure benannten. Die Forscher gingen davon aus, dass grundsätzlich alle Menschen einer Gesellschaft sich in die Diskussionen über die künftige Zielsetzung der Schule und des Unterrichts einbringen kön-

76 Freiburger Arbeitsgruppe für Lehrplanforschung, I97I.

77 Ebd. 
nen. Dass es überhaupt Ziele braucht und dass die Vielfalt der Meinungen und Ansichten der Leute einvernehmlich festgelegt und letztlich abgebildet werden könne, stand für die Forscher der FAL ausser Frage.

3. Die kantonalen Bildungsverwaltungen gewährleisten die Schulkoordination und pflegen den gegenseitigen Austausch von Informationen und Statistiken. Sie bedürfen noch zu entwickelnder Verfahren zur Sammlung, Gewichtung und Koordination von Zielsetzungen, die letztlich von einer zentralen Stelle aus garantiert werden.

Dieser dritte Bereich ist einerseits im Zusammenhang mit dem Schulkonkordat von $1970 \mathrm{zu}$ sehen, andererseits mit den Erkenntnissen aus der Forschung der FAL begründet, wonach die Schweizer Lehrplanlandschaft föderalismusbedingt von Vielfalt beziehungsweise von Unübersichtlichkeit geprägt war. ${ }^{78}$ Darauf sei konkordatsgemäss mit Koordinationsbemühungen und Informationsaustausch zu reagieren, wobei die Verantwortung hierfür bei den Bildungsverwaltungen verortet wurde. Es wurde zudem darauf hingewiesen, dass es für künftige Bildungsplanende eine Ausbildung an einer Universität geben müsse.79 Ferner sei der interkantonale Austausch bestimmten Zielen und Verfahren unterworfen. Es blieb aber die Frage offen, wer diese Ziele zu bestimmen habe.

4. Eine Schulreform ist eine permanente Aufgabe. Sie findet unter der Zuhilfenahme und Kontrolle wissenschaftlicher Berater statt. Schulinspektoren haben für die Informationsverbreitung und Formulierung von Vorschlägen eine grosse Bedeutung.

Der vierte Themenkomplex in den Thesen der FAL rückte die Wissenschaft und die Forschenden in eine einflussreiche Position. Demgemäss war Schulreform nicht ohne fachkundige Kontrolle und Beratung durchführbar. Schulreform wurde entworfen als Angelegenheit unter der Leitung der Wissenschaft. Wohl war den Schulinspektoren eine gewisse Bedeutung zugemessen worden. Sie beschränkte sich allerdings darauf, die Funktion eines Bindeglieds zu den Lehrpersonen einzunehmen.

Zusammenfassend verdeutlichen die Darstellungen und Analysen des publizierten Thesenpapiers der FAL die Vision einiger Wissenschaftler zur Planung und Organisation von Schulreform aufgrund curriculumbasierter Überlegungen. In ihren Augen war der politische Wille zur Schulkoordination anhand von zielorientierter Planung, Informationsbeschaffung und -austausch unter Einbezug aller Interessierten sowie - und dies ist in diesem Zusammenhang besonders bemerkenswert - mittels Beratung und Kontrolle der Wissenschaft zu

78 K. Frey, i968b.

79 Dieses Anliegen ist ab Oktober 1974 an der Universität Bern in Form eines Studienganges «Sachverständiger der Erziehungs- und Bildungswissenschaften» in die Tat umgesetzt worden. Weiterführend Aebli, I985; Regierungsrat des Kantons Bern, 1974. 
realisieren. Das Thesenpapier stellt schliesslich ein Dokument dar, woran die Haltung der Forschenden zu Organisation, Ziel und Dringlichkeit von Schulreformen abzulesen ist.

Die Mitglieder der FAL haben sich überdies weiterführend Gedanken gemacht dazu, wie wissenschaftliche Erkenntnisse anders als mittels Lehrerzeitschriften vermittelt werden könnten, und sich um die Sichtbarkeit ihrer Positionen ausserhalb der wissenschaftlichen Community bemüht.

\subsubsection{Leitideen der Öffentlichkeitsarbeit}

Im Januar 1972 verfassten vier Mitglieder der FAL ein arbeitsgruppeninternes Konzept zur «Öffentlichkeitsarbeit im Rahmen der Schweizerischen Bildungspolitik». ${ }^{8 \circ}$ Es handelte sich hierbei um ein Konzept, welches auf der Grundlage zweier Leitideen ihre Idealvorstellungen bildungspolitischer Öffentlichkeitsarbeit festhielt. Gemäss der ersten Leitidee sollten bildungspolitische Entscheidungen dahingehend vorbereitet werden, dass «den Entscheidungen eine differenzierte und rationale Beurteilung der bildungspolitischen Probleme vorausgeht, in adäquatem Masse finanzielle Mittel zur Lösung solcher Aufgaben bereitgestellt werden [und] die Ausbildung von Fachleuten im pädagogischen Sektor qualitativ und quantitativ gefördert wird». ${ }^{81}$ Nach Auffassung der FAL mangelte es der Bildungspolitik in der Schweiz zu Beginn der I970er-Jahre an tatsachengebundener und nüchterner Entscheidungsfindung, den finanziellen Mitteln zur politischen Bearbeitung aktueller Herausforderungen im Bildungsbereich sowie an einer Ausbildung qualifizierter Bildungsexpertinnen und -experten. Um diesen Umstand zu optimieren, müssten, so die zweite Leitidee, «die durch bildungspolitische Entscheidungen Betroffenen (Gruppen und Individuen) [...] urteilsfähiger werden, um kompetent an den Entscheidungen teilnehmen zu können und dadurch zu guten Lösungen beizutragen». ${ }^{82}$ Diese beiden Ideen verstanden die Mitglieder der FAL auch als Zielsetzung und folgerten, dass zur Verbesserung der festgestellten Umstände «Informationen vermittelt werden müssen», wobei «versucht werden soll, über adäquate Informationsträger unsere Kenntnisse und Konzepte weiterzugeben». ${ }^{8_{3}}$

Das Konzeptpapier führte eine ganze Liste potenzieller Adressaten auf, welche mit zusätzlichen Informationen zu bildungspolitischen Themen versorgt

80 Aregger, Brunner, Isenegger, Santini, FAL-Konzept zur Öffentlichkeitsarbeit im Rahmen der Schweizerischen Bildungspolitik, Januar I972, FAL-Archivalien.

8 I Ebd., S. I.

82 Ebd.

83 Ebd. 
werden sollten. Es wurde klar zwischen zwei Personengruppen unterschieden: jenen, welche bereits anlässlich einzelner Projekte mit ihnen in Austausch standen, und den neu mit Informationen zu Versorgenden. Grob zusammengefasst beabsichtigten die FAL-Mitglieder, politische Entscheidungsträger, Akteure aus der Schulpraxis sowie private und privatwirtschaftliche Verbände $\mathrm{zu}$ adressieren. Konkret genannt wurden beispielsweise der Wissenschaftsrat, EDK-Kommissionen, eidgenössische Räte, Inspektoren, Schulleiter, Parteien, Eltern- und Lehrerverbände, privatwirtschaftliche Bildungsinstitute wie die Migros oder private Institute wie die Katholische Arbeitsgemeinschaft für Erwachsenenbildung der Schweiz und Liechtensteins.

Allerdings war gemäss dem Konzept weit mehr angedacht als blosse Informationsvermittlung. Es sollten «die folgenden Einstellungen gegenüber Problemen, Innovationen, schulreformerischen Tätigkeiten gefördert werden»: ${ }^{8}$

- Primat der Fachautorität berücksichtigen

- zu rationalisierten Entscheidungen tendieren

- zur Demokratisierung von Entscheidungsprozessen tendieren

- innovationsbereit sein

- bereit sein, eigene Leistungen zu relativieren

Die Informationen hatten zum Ziel, bei den Adressaten etwas auszulösen, bestimmte Einstellungen zu fördern, eine Offenheit gegenüber Neuerungen zu erwirken, und es war beabsichtigt, dies an bestimmten Lernbereichen festzumachen. Das Konzeptpapier hatte einige zu erwerbende Kenntnisse lernzielartig festgehalten. Es war vorgesehen, die Adressaten zum Lernen anzuregen, sodass sie «Kenntnisse besitzen über:

- Feststellungen, Prinzipien, Methoden und Arbeitsformen der Curriculumforschung,

- die Lehrerrolle bei der Schulreform,

- die Funktion der Bildungsforschung,

- Probleme des heutigen Schulsystems,

- den Zusammenhang zwischen Schule und Gesellschaft.»85

Die FAL-Mitglieder stellten anlässlich ihres Konzeptpapiers auch Überlegungen dazu an, wie die Informationen am angemessensten zu vermitteln seien. Sie formulierten entsprechende methodische Prinzipien wie «Aktualitätsbezug, problemorientierte Darstellungen oder Berücksichtigung des Sprachniveaus der Adressaten». ${ }^{86}$ Ferner umfasste das Konzept eine ausführliche Liste zu möglichen Zeitungen und Zeitschriften als «Informationsträger» und es enthielt Vorgaben dazu, in welcher Frequenz die Zeitungen mit Informationen

84 Ebd., S. 2.

85 Ebd.

86 Ebd. 
bespielt werden sollten. Zur besseren Übersicht und Planung des Vorhabens wurde zudem ein Koordinator aus den Reihen der FAL-Mitarbeiter ernannt. Seine Aufgabe umfasste das Anlegen eines Archivs oder die Evaluation der Öffentlichkeitsarbeit der Arbeitsgruppe.

Das beschriebene Konzept zur Öffentlichkeitsarbeit verdeutlicht anschaulich bestimmte Absichten und Ziele der Curriculumforscher aus Freiburg in den frühen I970er-Jahren. Sie wollten auf unterschiedliche Akteure des Bildungswesens in der Schweiz Einfluss nehmen, indem sie systematisch ihre Standpunkte in Aufsätzen und Beiträgen publizierten. Die FAL-Mitglieder gingen davon voraus, dass die Leserinnen und Leser für ihre bildungspolitischen Vorstellungen und Ansichten zu gewinnen wären, wenn sie über die bestehenden Probleme und Herausforderungen aufgeklärt würden. Dazu legten sie sich eine differenzierte Vorgehensweise zurecht, welche nicht bloss geschriebenes Wort bleiben sollte.

Im August 1973 wandte sich die Freiburger Arbeitsgruppe brieflich an nicht weniger als 22 Redaktionen von Zeitungen und Zeitschriften aus der ganzen Schweiz. Diese wurden eingeladen, am 28. August an einem «Informationsund Diskussionsnachmittag für Redaktoren und Journalisten zu Fragen der schweizerischen Bildungspolitik» ${ }^{87}$ teilzunehmen. Als Grund für die Einladung wurde das Ziel der FAL beschrieben, nicht nur wissenschaftliche Erkenntnisse zu erarbeiten, sondern auch «reale Verbesserungen der Schule» anzustreben. ${ }^{88}$ Aus diesem Grund müsse auch die breitere Öffentlichkeit angesprochen werden, denn «wir [möchten] eine wirkungsvolle Informationspolitik betreiben, die auch politische Gruppen und Entscheidungsträger einbezieht».89

Der Einladung lag ein zweiteiliges Programm bei, wonach zunächst eine Präsentation der aktuellen Reformprojekte der FAL geplant war, anschliessend eine Diskussionsrunde mit den Journalisten. Für die Diskussion schlug die FAL einige Fragen vor, beispielsweise: «Welche Ratschläge können Journalisten einem Bildungsforschungsinstitut für eine wirkungsvolle Öffentlichkeitsarbeit geben?» Oder: «Welches sind die Erwartungen der Leser an Artikel, die Aussagen zum Bereich der Bildung beinhalten?»90

Das Schreiben an die Redaktionshäuser kann als Beleg dafür gesehen werden, dass die Wissenschaftler ihre Absichten strategisch in die Tat umzusetzen gedachten. Sie wollten an die mediale Öffentlichkeit gelangen, um ihr Konzept der Öffentlichkeitsarbeit einzulösen. Sie waren der Überzeugung, ihre Leitide-

87 Joe Brunner: Informations- und Diskussionsnachmittag für Redaktoren und Journalisten zu Fragen der schweizerischen Bildungspolitik, 7. 8. I973, FAL-Archivalien.

88 Ebd.

89 Ebd.

90 Ebd. 
en zu aktuellen bildungspolitischen Fragen über die Printmedien vermitteln zu können, und waren sich sicher, damit zur Verbesserung der Volksschule in der Schweiz beizutragen. Nicht zuletzt knüpften sie Kontakte mit den Medien, um in Erfahrung zu bringen, wie die von ihnen angestrebte Zusammenarbeit am besten auszugestalten wäre.

Bevor im Anschluss systematisch Orte, Themen und Diskussionsstränge der bildungspolitischen Debatten zu Curriculumreformen genannt werden und aufgezeigt wird, inwiefern die Curriculumforschenden sich an den dortigen Debatten beteiligten, wird zuerst ein kurzes Fazit zur Programmatik der Curriculumforschung gezogen und daraus resultierende bildungspolitische Aspirationen werden offengelegt.

\subsubsection{Curriculumreform nach wissenschaftlichem Diktum}

Parallel zu ihren Qualifikationsarbeiten im Bereich der Curriculumforschung in der Schweiz ${ }^{11}$ brachten sich die Forschenden mit ihren Positionsbezügen in Fach- und Lehrerzeitschriften nicht nur in die aktuellen wissenschaftlichen Diskussionen ein, sie bezogen darüber hinaus Stellung in den bildungspolitischen Debatten zur Bewältigung der anstehenden Schulkoordinationsbemühungen zwischen den Kantonen. Ihre Forschungsergebnisse zu Curricula, deren Konstruktion sowie Implementation, anerboten sie als politisches Planungs- und Steuerungsinstrument und stellten in Aussicht, die vorliegenden Probleme rational und wissenschaftsbasiert zu lösen. Curriculumforschung ist zu Curriculumpolitik geworden.

Ungeachtet der ungeklärten Konstruktionsprobleme von lernzielbasierten Curricula legitimierten die Wissenschaftler ihre Lösungsvorschläge, indem sie mit Bezug auf die eigenen Ansätze die Modernisierung der Volksschule propagierten. Die bisherigen Lehrpläne wurden als überaltert taxiert und für eine zeitgemässe Unterrichtsplanung als unbrauchbar erklärt. Mit dieser Kritik bespielten die Forscher die gesellschaftlich vorherrschende Planungsgläubigkeit und bedienten den Wunsch nach und das Ideal von Fortschritt. Sie kritisierten das Bisherige, verorteten ihre Arbeit in aktuellen bildungspolitischen Bestrebungen und zeigten im selben Atemzug den einzuschlagenden Lösungsweg auf.

Bemerkenswert am strategischen Vorgehen der Mitglieder der Freiburger Arbeitsgruppe ist ihr Konzept der Wissenschaftskommunikation. Sie gingen davon aus, dass ihre Vorschläge zur Schulreform als Curriculumreform auf

9I Vgl. dazu Kapitel zwei. 
breitere Akzeptanz stossen würden, wenn sie einer innovativen Informationsstrategie gemäss Aufklärungsarbeit über unterschiedliche Kanäle leisten würden. Sämtliche Involvierten und an Entscheidungen Beteiligten sollten folglich mit Informationen der FAL versorgt werden, damit Reform- und Innovationsbereitschaft sowie eine grundlegende Kenntnis des Schulsystems aufgebaut werden könnten.

Die Rekonzeptualisierungen des Forschungsgegenstandes der FAL für bildungspolitische Zusammenhänge haben zusammen mit den formulierten Absichten über aufzubauende «Einstellungen» gegenüber schulpolitischen Belangen deutlich die Rolle und Funktion veranschaulicht, welche sich die Wissenschaftler in den Reformprozessen selbst zuschrieben. Die Forschenden ordneten sich konzeptionell in den zu lancierenden Reformen als federführende Akteure ein, ausgestattet mit Entscheidungsmacht und legitimiert durch Expertenwissen. Sie sollten in den zirkulär angelegten Reformprojekten als Bildungsexperten begleitend, beratend und steuernd in Erscheinung treten. Die Mitglieder der FAL zielten darauf ab, sich selbst im bildungspolitischen Feld gleichzeitig als Experten, Berater und operative Leiter von aus ihrer Sicht dringlichen Reformen zu positionieren.

\subsection{Das Curriculum in bildungspolitischen Debatten}

Über mehr oder weniger zufällige Kontakte zwischen Sozialwissenschaftlern und Politikern bzw. Regierungsbeamten hinausgehende Formen institutionalisierter sozialwissenschaftlicher Politikberatung existierten kaum.92

Anders als im oben stehenden Befund zu den i960er-Jahren wird anhand des vorliegenden Teilkapitels deutlich, dass zur Bearbeitung bildungsspezifischer Problemstellungen der i970er-Jahre Personen aus Wissenschaft, Verwaltung, Politik und Schulpraxis anlässlich von Konferenzen, Seminaren sowie länger dauernden Projekten zusammengeführt worden sind. Allen nachfolgend benannten und beschriebenen Gremien gemeinsam war, dass die dort geführten Debatten inhaltlich an den Gegenstand der Curriculumforschung anschlossen. Es wäre aber verfrüht, die hier analysierten Anlässe des Wissensaustausches über Curricula bereits darauf zu befragen, inwieweit explizit Forschungsresultate der Curriculumforschung in den Debatten verhandelt wurden oder inwiefern diese Wissensbestände in den Debatten eine Veränderung erfahren hätten. Das Ansinnen dieses Kapitels besteht vielmehr darin, Curricula zunächst als 
Debattengegenstand aufzuspüren sowie die beteiligten Akteursgruppen, die Hintergründe der Debatten und ihren Verlauf aufzuzeigen.

Die in diesem Kapitel präsentierten Anlässe des Wissensaustausches unterliegen zwangsläufig einer quellenbedingten Auswahl. Die Auswahl konzentriert sich vorrangig auf Formate des Austausches auf interkantonaler und nationaler Ebene, um gewissermassen die grossen Linien der Debatten in der Schweiz verfolgen zu können. Hierzu wird auf Publikationen und Berichte der Schweizerischen Koordinationsstelle für Bildungsforschung (SKBF) und der EDK zurückgegriffen. Überdies werden auch Verweise in den Archivalien der Freiburger Arbeitsgruppe auf die Beteiligung ihrer Mitarbeiter in solchen Gremien beigezogen.

Thematisch strukturiert sind die nächsten vier Unterkapitel wie folgt: Zunächst werden Reformdebatten in der Lehrerinnen- und Lehrerbildung der 1970erJahre beleuchtet, dann wird der Blick auf einen Moment der Formierung der Erziehungs- und Bildungsforschung geworfen, um anschliessend einige Projekte unter Anleitung der pädagogischen Kommission der EDK in den Fokus zu rücken. Am Schluss werden Analysen zu den Referatstätigkeiten der Freiburger Arbeitsgruppe für Lehrplanforschung dargelegt. Sie veranschaulichen im Sinne einer Ergänzung das Bemühen der FAL, alle Interessierten über ihre Forschungsresultate zu informieren. Das Teilkapitel schliesst wiederum mit einem Fazit ab.

\subsubsection{Reformdebatten der Lehrerinnen- und Lehrerbildung}

Wie soll aus der Sicht von Volksschullehrpersonen die Ausbildung ihrer künftigen Berufskolleginnen und -kollegen inhaltlich ausgestaltet sein? Dieser Frage widmete sich ein Forschungsprojekt unter dem Titel «Bildungsbedürfnisse der Volksschullehrer» (BIVO) im Auftrag des Schweizerischen Pädagogischen Verbandes (SPV) und der Konferenz der Leiter Schweizerischer Lehrerbildungsanstalten in den Jahren 1969-1974. Das pädagogische Institut der Universität Zürich wurde mit diesem Projekt in Zusammenarbeit mit dem pädagogischen Institut der Universität Freiburg betraut. In Freiburg arbeitete Urs Isenegger von der Freiburger Arbeitsgruppe an dem Projekt, wo er vornehmlich die methodischen Grundlagen im Bereich der Lernzielformulierung verantwortete. ${ }^{33}$ Der Curriculumforschung fiel im BIVO-Projekt also die Aufgabe zu, ein wissenschaftliches Instrumentarium zur Festlegung von Ausbildungsinhalten angehender Lehrpersonen zu entwerfen. ${ }^{94}$

$93 \mathrm{Zu}$ seiner aus dem Projekt entstandenen Dissertation vgl. das Vorkapitel und Isenegger, 1972.

94 Im Laufe des Projektes wurde eine Vielzahl weiterer förderungswürdiger Fragen aufgeworfen, was die Zürcher Erziehungsdirektion veranlasste, das Projekt um vier Jahre zu 
Zur selben Zeit initiierten der SPV und die Konferenz der Leiter Schweizerischer Lehrerbildungsanstalten eine Tagung mit über 30 Vertreterinnen und Vertretern aus Wissenschaft und Lehrerbildung in Kaltbad auf der Rigi. Der Präsident der Konferenz der Leiter Schweizerischer Lehrerbildungsanstalten formulierte eindringlich das Ziel der Tagung im Oktober 1969: Die Lehrerinnen- und Lehrerbildung in der Schweiz sei in einen «Teufelskreis» hineingeraten, welcher sich konstituiere durch einen Mangel an Lehrpersonen und eine ungenügende Ausbildung der Lehrpersonen. Dieser Teufelskreis müsse überwunden werden. ${ }^{95}$ Der Herausgeber des im Jahr darauf erschienenen Tagungsbandes präzisierte, auf welchem Weg diese Herausforderung an der Tagung angegangen worden sei: Es gehe «um Fragen der Funktion der Pädagogik in der Lehrerbildung, um ihren Inhalt, um Probleme des Aufbaus und der Anordnung, kurz, um Probleme des Curriculums». ${ }^{6}$ Ferner habe man sich aufgrund der Reformerfahrungen im Ausland darüber verständigt, die Planung der anzustrebenden Reformen in der Lehrerinnen- und Lehrerbildung primär mittels einer Neujustierung ihrer Inhalte voranzubringen.97

Der Ausgangspunkt der Debatten auf der Rigi wurde massgeblich bestimmt von einem zweiteiligen Bericht mit dem Titel «Der Ausbildungsgang der Lehrer. Eine Modellanalyse des Unterrichts in den 52 Lehrerbildungsanstalten zum Zwecke der Curriculumreform».9 ${ }^{8}$ Verfasst haben diesen Bericht Karl Frey und seine Mitarbeiter von der Freiburger Arbeitsgruppe gemäss einem 1967 erteilten Auftrag der Tagungsorganisatoren. Der Bericht schliesst mit einer Empfehlung, die die Bedeutung und Funktion eines neuen Curriculums und damit der neu zu konzipierenden Inhalte der künftigen Ausbildungsgänge für Lehrerinnen und Lehrer zusätzlich unterstreicht: «Die vielerorts angestrebte Verlängerung der unmittelbar berufsvorbereitenden Phase im OS [Oberseminar, Anm. L. H.] auf 4 oder 6 Semester (und damit die Einrichtung der Lehrerbildung auf Akademiebzw. Hochschulebene) folgt nach der Entwicklung eines neuen Curriculums. Die Konstruktion des neuen Curriculums setzt ihrerseits das Erlernen von didaktischen Arbeitstechniken, Methoden der Unterrichtsvorbereitung und von Strategien der Innovation (Verwirklichung der Reform) voraus.»

Die Mitglieder der FAL als Urheber des Berichtes zum Ausbildungsgang angehender Lehrpersonen und als Mitverantwortliche des BIVO-Projektes waren an der Tagung auf der Rigi zahlreich vertreten und verantworteten im Tagungs-

verlängern und explizit dafür eine neue Assistenzstelle am Zürcher Institut zu schaffen. Vgl. StAZH, RRB Nr. 5384 vom 5. I I. 1970.

95 Gehrig, I970a, S. 8.

96 Gehrig, 1970b, S. 9. Hervorhebungen im Original.

97 Zum «Ausland als Argument» vgl. Gonon, 1998; Zymek, I975.

98 K. Frey, 1969b. 
band mit ihren Vorstellungen von der Neukonzeption der Ausbildung angehender Lehrpersonen rund die Hälfte der Beiträge. Indes konnte an der Tagung hinsichtlich der Frage, was für Reformen einzuleiten seien, keine abschliessende Einigung erzielt werden. So beantragte die Konferenz der Leiter Schweizerischer Lehrerbildungsanstalten bei der EDK erfolgreich die Schaffung einer Expertenkommission «Lehrerbildung von morgen» (LEMO). 99

Die LEMO-Expertenkommission machte es sich zur Aufgabe, «über die Einigung auf den Bildungsauftrag und auf das Bildungsprogramm der schweizerischen Lehrerbildungsanstalten eine Verbesserung und eine Harmonisierung der künftigen Lehrerbildung in der Schweiz zu erreichen». ${ }^{100}$ Ab I 970 tagten Vertreterinnen und Vertreter aus der Forschung, der Lehrerbildung und der Volksschule regelmässig, um 1975 ihren Bericht vorzulegen. Dieser sollte von nachhaltiger Bedeutung bleiben, zumal selbst zehn Jahre später festgehalten wurde, dass «auch heute noch kaum einer, der sich in unserem Land mit Lehrerbildungsfragen befasst, an ihm» vorbeikomme. ${ }^{\text {or }}$ Allerdings ist auch im Zuge der LEMO-Kommission ein konsensuales Ergebnis in Bezug auf die Weiterentwicklung der Lehrerbildung in der Schweiz erzielt worden, ohne eindeutige Stossrichtungen vorzugeben.

Die Ursache für den vorgelegten «Lösungs-Pluralismus» ${ }^{102}$ verortete Anton Strittmatter als ehemaliges Kommissionsmitglied in der Kommissionzusammensetzung, welche ein Spannungsverhältnis zwischen drei Positionen provoziert habe: ${ }^{103}$ Hans Gehrig ${ }^{104}$ als Leiter des BIVO-Projektes wollte das künftige Curriculum angehender Lehrpersonen an Anwendungsproblemen im Berufsalltag und kritischen Berufssituationen ausrichten. Hans Aebli zog es vor, die Themenbereiche in der Lehrerbildung anhand von begrifflichen Strukturen $^{\text {1os }} \mathrm{zu}$ fassen, und kritisierte deutlich die Auffassung von Karl Frey. Dieser argumentierte vor dem Hintergrund der Forschungen in der FAL für ein Curriculum mit präzisen Lernzielen. ${ }^{106}$

Mit dem Expertenbericht «Lehrerbildung von morgen» waren die Debatten um das Curriculum und die Struktur der künftigen Lehrerinnen- und Lehrerbildung in der Schweiz nicht abgeschlossen. Bereits zwei Jahre später fanden

Criblez, 2002b.

Müller, I975, S. I 5 .

Müller, I985, S. I I.

Ebd., S. Is.

Strittmatter, 2003 .

Hans Gehrig war I97 I-I980 Direktor des Oberseminars in Zürich, anschliessend Direktor des neu geschaffenen Seminars für Pädagogische Grundausbildung bis i 988, um dann bis I994 das Direktorium des Pestalozzianums in Zürich zu übernehmen.

Weiterführend Aebli, r96ı; I97ı.

Für weiterführende Informationen zu LEMO Huber, 2017. 
sich auf Initiative der pädagogischen Kommission der EDK vom 20. bis 23. September 1977 über 70 Expertinnen und Experten aus Lehrerbildung, Forschung, Schulpraxis, Bildungsverwaltung und Bildungspolitik in Luzern ein, um über die Lehrerbildung in der Schweiz und ihre Auswirkung auf den Unterricht zu diskutieren. ${ }^{107}$

Der damalige Präsident der EDK, Alfred Gilgen, legte das Gewicht seines einleitenden Beitrages auf die Bedeutung der Aus- und Weiterbildung der Lehrpersonen für die laufenden Schulreformen. Er begründete: «Keine Schulreform kommt ohne die Lehrerschaft zum Durchbruch, ja sie vollzieht sich recht eigentlich erst in der Schulwirklichkeit. Damit werden auch Schulreformen zu Anliegen der Lehreraus- und weiterbildung.» ${ }^{108}$ Mit dieser Position bereitete Gilgen Referierenden wie Karl Frey und Kurt Aregger den Boden für ihre Ausführungen. Sie plädierten für einen Ausbau von Anteilen an praktikumsbezogenem, forschendem Lernen in der Ausbildung ${ }^{109}$ oder für eine «Pädagogisierung der Fachdisziplinen» in der Ausbildung angehender Lehrpersonen, gewissermassen eine Fachdidaktisierung der Unterrichtsinhalte. ${ }^{\text {10 }}$

Die meisten an der Expertentagung vom September 1977 Referierenden machten die Auseinandersetzung mit dem Curriculum in der Lehrerinnen- und Lehrerbildung an der Frage der Qualifizierung künftiger Lehrpersonen fest. Wenn Klarheit über die Anforderungen an die Lehrpersonen im Schulalltag herrschte, könnte sich das Curriculum der Ausbildung daran ausrichten, so die Grundüberlegung in einigen Referaten. Auch Urs Peter Lattmann verwies auf diese «wohl kaum bestrittene» Vorgehensweise, zeigte aber ebenso auf, dass die Forschenden «gegenwärtig noch über zu wenige Erkenntnisse verfügen würden, um auf dieser Basis eine curriculare Planung der Lehrerbildung vornehmen zu können». ${ }^{I I}$

Als letztes bedeutsames Beispiel für Debatten in der Lehrerbildung mit Bezügen zu Themen der Curriculumforschung sei eine Expertenkommission der Innerschweizer Erziehungsdirektorenkonferenz (IEDK) erwähnt, welche sich ab dem Frühjahr 1976 auch mit curricularen Planungsfragen beschäftigte. Ihr Ziel bestand in der Konzipierung einer Reorganisation der Ausbildung künftiger Lehrpersonen für die Volksschuloberstufe in der Zentralschweiz unter dem Titel «Lehrerbildung für die Orientierungsstufe». Die erste von drei Leitfragen der Expertenkommission lautete demgemäss: «Auf welches Berufsbild, auf welche Qualifikationen hin sind künftige Orientierungslehrer auszubilden?» ${ }^{112}$

I07 Die Tagung firmierte unter dem Namen «Expertentagung Lehrerbildung und Unterricht» (ELEBU).

Io8 Aregger, Lattmann und Trier, 1978, S. 6.

I09 Aregger, 1978.

I IO K. Frey, 1978, S. 35 .

I I Lattmann, I978, S. I00 f.

I 2 Strittmatter, I978c, S. 5. 
Die Kommission knüpfte an Vorarbeiten der vorhergehenden Kommission Rickenbacher an und war gleichzeitig verzahnt mit Planungsvorhaben für eine neue Hochschule im Aargau und eine Universität in Luzern. ${ }^{133}$ Dementsprechend war die Kommission besetzt worden mit Rektoren von Lehrerseminaren, Kantonsschullehrern, Vertretern von kantonalen Planungsstellen sowie Delegierten für Hochschulfragen vonseiten der kantonalen Verwaltungen und des Bundes. Zahlreich in der Kommission vertreten waren auch aktuelle und ehemalige Mitglieder der FAL. Der Zentralschweizer Beratungsdienst für Schulfragen (ZBS) leitete und betreute die Kommission.

Die Kommission erarbeitete einen Expertenbericht und formulierte darin über 40 Thesen, welche sich zu den «Leitideen für die Orientierungsstufe in der Zentralschweiz», zum «Verhältnis von Schulreform und Lehrerbildung für die Orientierungsstufe», zum «Berufsbild des Lehrers in der Orientierungsstufe» und schliesslich zu «Grundsätzen für die Gestaltung der Lehrerausbildung für die Orientierungsstufe» äusserten. ${ }^{114}$ Etliche der vorgeschlagenen Entwicklungen nahmen Überlegungen und Konzepte der Freiburger Curriculumforschung auf. In Bezug auf den Unterricht schlug das Konzeptpapier etwa vor, Rahmenlehrpläne für die Orientierungsstufe zu erarbeiten und die Lehrmittel darauf abzustimmen. Zudem sollten die künftigen Lehrpersonen auch «die individuellen Eingangsvoraussetzungen der Schüler abklären sowie den Unterrichtserfolg überprüfen und zur Verbesserung der Unterrichtsplanung auswerten». ${ }^{\text {II }}$ Im Januar 1977 verabschiedete die IEDK den Bericht der Expertenkommission und leitete auf dessen Grundlage eine umfassende Reform der Orientierungsstufe in der Zentralschweiz ein.

\subsubsection{Formierungsdebatten der Erziehungs- und Bildungsforschung}

Die Schweizerische Koordinationsstelle für Bildungsforschung (SKBF) veranstaltete Ende Oktober 1974 eine Arbeitstagung unter dem Titel «Die Bildungsforschung im Rahmen des Forschungsberichtes des Schweizerischen Wissenschaftsrates (SWIR)». Anlass dieses Treffens von Vertreterinnen und Vertretern aus Wissenschaft, Bildungsverwaltung, Lehrerinnen- und Lehrerbildung und weiteren Interessierten war die «auch vom Schweizerischen Wissenschaftsrat festgestellte mangelhafte Entwicklung der Bildungsforschung in der Schweiz» ${ }^{116}$ Der Schweizerische Nationalfonds stellte nicht zuletzt aufgrund dieses Befun-

I 3 Huber, 2017.

I 4 Strittmatter und Winiger, 1978.

i Is Ebd., S. I I.

I 6 Kägi, 1974. 
des einen Anteil der projektierten 80 Millionen Franken für die Sonderförderung der Erziehungs- und Bildungsforschung in Aussicht. Infolgedessen rief Urs Hochstrasser ${ }^{17}$ die Teilnehmenden dazu auf, bis 1975 geeignete Forschungskonzepte einzureichen. ${ }^{118}$

Die SKBF beschrieb diese Tagung als erste Zusammenkunft einer grösseren Zahl von Vertreterinnen und Vertretern der Bildungsforschung. Im zugehörigen Tagungsband ${ }^{119}$ formulierte sie die aus ihrer Sicht drei wichtigsten Ergebnisse der Versammlung: Die Tagung habe erstens dazu beigetragen, grundlegende Probleme zu erörtern. Besonders bedeutsam gewesen sei die Diskussion des Theorie-Praxis-Bezuges und die damit verbundene Forderung nach einem Einbezug der Praxisebene in die Bildungsforschung. Insbesondere zu diesem Punkt hatten Kurt Aregger und Karl Frey als ehemalige Mitglieder der FAL einiges beizutragen. Sie erhielten Gelegenheit, ihre Standpunkte aus der Perspektive der Curriculumforschung zu präsentieren. Die beiden Referate dürften den anwesenden Journalisten der «Neuen Zürcher Zeitung» in seiner Wahrnehmung bestärkt haben, wonach «die eigentlichen Erziehungsprobleme in den Hintergrund gedrängt [sind], während die Lernprozesse kognitiver Art (intellektuelle Komponente) das Feld zu beherrschen scheinen». ${ }^{120}$ Zweitens wurden an der Tagung einhellig die Bedeutung und das Erfordernis eines Entwicklungsplanes für die Bildungsforschung in der Schweiz festgehalten, wobei diesem Bedürfnis erst 1988 entsprochen wurde. ${ }^{\text {I2I }}$ Und drittens beauftragten die Versammelten eine Arbeitsgruppe mit der Gründung der Schweizerischen Gesellschaft für Bildungsforschung (SGBF). Dieser Entschluss wurde im Folgejahr realisiert. Der erste Kongress der SGBF knüpfte notabene wieder am ersten der Kernthemen der Tagung an. In den Worten des ersten Präsidenten der SGBF bestand es darin, «à savoir la relation entre la recherche et la pratique, entre le chercheur, l'enseignant et l'administration». ${ }^{122}$

I 7 Urs Hochstrasser wurde 1969 vom Bundesrat zum Leiter der Abteilung für Wissenschaft und Forschung (später Bundesamt für Bildung und Wissenschaft) berufen. Er verantwortete in dieser Funktion bis 1989 die Wissenschaftspolitik auf Bundesebene.

I 8 Aus dem zweiten Kapitel ist bereits hervorgegangen, dass das erste nationale Forschungsprogramm im Bildungsbereich «Probleme der sozialen Integration in der Schweiz» zum Thema hatte.

I I9 Schweizerische Koordinationsstelle für Bildungsforschung, I975.

I 20 Kägi, I 974 .

I 2 I Schweizerische Gesellschaft für Bildungsforschung, I988.

I22 Goldschmid, 1977, S. 6. 


\subsubsection{Bildungsplanung, Bildungsforschung und Schulpraxis im Austausch}

«Des Weiteren wird zur Zeit in der Pädagogischen Kommission die Frage besprochen, ob und in welcher Weise Kantone oder Regionen bei der Erstellung von Lehrplänen zusammenarbeiten könnten.» ${ }^{123}$ Anhand dieses Zitats aus dem Jahresbericht der EDK von 1974 wird deutlich, dass auf interkantonaler Ebene eine Kommission bestand, welche sich mit bestimmten pädagogischen Geschäften auseinandersetzte, die auch die kantonalen Lehrpläne und deren Revision betrafen. Gegründet wurde die pädagogische Kommission (PK) im Juli I967 mit dem Ziel, die EDK in allen Belangen hinsichtlich der anzustrebenden Koordination des Schweizer Schulwesens zu beraten. Zunächst ging es darum, konkrete Vorschläge betreffend den Schuljahresbeginn, das Schuleintrittsalter und den Fremdsprachenunterricht auf der Volksschulstufe auszuarbeiten und der EDK vorzulegen. Nachdem das Schulkonkordat betreffend die Koordination I97I in Kraft getreten war, wurde die PK aufgelöst, 1972 aber bereits wieder eingeführt. Die konkreten Aufgaben bestanden nun I. in einer Bestandsaufnahme und Bedürfnisabklärung in den Regionen und Kantonen, 2. der Erarbeitung und Formulierung von Mandaten für Institutionen, Kommissionen und Beauftragte der EDK, 3. der Koordination und Überwachung der laufenden Geschäfte im pädagogischen Bereich, 4. der Begutachtung der Anträge und Arbeiten von Institutionen, Kommissionen und Beauftragten und 5. der Verarbeitung von Vernehmlassungsergebnissen. ${ }^{\mathrm{I} 2}$

Die PK war besetzt mit je einer Vertretung aus den vier EDK-Regionen, ${ }^{125}$ sechs "pädagogische[n] Experten» ${ }^{126}$ und, um die Lehrpersonen angemessen in die Debatten mit einzubeziehen, mit acht Vertretern aus der Konferenz Schweizerischer Lehrerorganisationen (KOSLO). ${ }^{127}$ In dieser Zusammensetzung behandelten die Kommissionsmitglieder koordinationsrelevante Geschäfte, wobei ab I 975 unter dem Schlagwort «Koordination durch Kooperation» weitere Formen der Zusammenarbeit der Kantone geprüft wurden. ${ }^{128}$ Diese Strategie ist

I 23 Konferenz der kantonalen Erziehungsdirektoren, 1974, S. I7.

I 24 S. n., I 973 .

I 5 Die Kantone haben sich zusätzlich in vier Regionalkonferenzen zusammengeschlossen, wobei die Westschweizerische EDK (mit den Kantonen BE, FR, TI, VS, VD, NE, GE, ab I 979 JU), die Nordwestschweizer EDK (BE, LU, FR, SO, BS, BL, AG), die Innerschweizer EDK (LU, UR, SZ, OW, NW, ZG, VS) und die Ostschweizerische EDK (ZH, GL, SH, AR, AI, SG, GR, TG, Fürstentum Liechtenstein) unabhängig voneinander tagten.

I $26 \mathrm{Zu}$ den «Experten» zählten vorrangig Vertreter aus pädagogischen Abteilungen oder Planungsstellen einzelner Kantone, vgl. dazu die Nennung der Mitglieder in den Jahresberichten der EDK, hier exemplarisch Konferenz der kantonalen Erziehungsdirektoren i 975, S. 49.

I 27 S. n., I 1973 .

I 28 Konferenz der kantonalen Erziehungsdirektoren, I975, S. 7. 
auch innerhalb einzelner EDK-Regionen angestrebt worden, wie einzelne Bemühungen um Rahmenlehrpläne zeigen. Anlässlich der oben erwähnten Volksschulreform in der Zentralschweiz von der Oberstufe zur Orientierungsstufe rückten die Lehrpläne der beteiligten Kantone in den Fokus. Es wurde auf Antrag und «entsprechend den Vorschlägen des Leiters des Zentralschweizerischen Beratungsdienstes für Schulfragen beschlossen», die Lehrpläne auf der Orientierungsstufe in der IEDK weitgehend zu harmonisieren. ${ }^{\mathrm{I} 29}$ Die personelle Zusammensetzung der PK erlaubte offenbar eine breite und nachhaltige Debatte zu den jeweiligen Schwerpunktthemen und begünstigte die Entstehung eines Netzwerks, «das sehr viel in informeller Weise zur besseren Koordination der kantonalen Schulsysteme beitrug», so Carlo Jenzer. ${ }^{130}$

$\mathrm{Ab}$ Mitte der 1970er-Jahre intensivierte die PK ihre Koordinationsintentionen auf der Grundlage von Lehrplanrevisionen. Sie initiierte diverse Vorarbeiten, an welchen die Mitglieder der FAL massgeblich beteiligt waren. Der Auftrag an die FAL bestand darin, wie einst im Rahmen der Dissertation von Karl Frey, ${ }^{\mathrm{I} 1 \mathrm{I}}$ eine Übersicht über die Lehrplanvielfalt in der Schweiz zu erarbeiten, um weiterführende Debatten zu ermöglichen. Für dieses Geschäft und zur Organisation einer entsprechenden Tagung zum Thema «Lehrplangestaltung, Reform und Koordination» schuf die PK eigens eine Arbeitsgruppe. ${ }^{132}$ Der Jahresbericht der EDK von 1977 vermeldete rückblickend: «Die Tagung befasste sich mit dem Stand der derzeitigen kantonalen Lehrpläne sowie mit der Reform der Lehrpläne, den Möglichkeiten zur interkantonalen Zusammenarbeit und den konkreten Wegen zur Revision von Lehrplänen. ${ }^{133}$

I978 publizierte die EDK ihr Jahrbuch unter dem Titel «Schulkoordination über Lehrplanreform». Es fungierte zudem als Tagungsband der Veranstaltung von 1976 und betonte einleitend: «Insbesondere aber möchte das Werk zur Erneuerung von Lehrplänen und zu engerer Zusammenarbeit unter den Kantonen Wege weisen.» ${ }^{134}$ Diese Einschätzung von Eugen Egger ${ }^{135}$ wird nachfolgend mit ausgewählten Eckpunkten aus dem Jahrbuch der EDK kontextualisiert.

I29 Ebd., S. 36.

I30 Jenzer, I995, S. 38. Carlo Jenzer studierte Pädagogik, Philosophie und französische Literatur und doktorierte über Michel de Montaigne. Er leitete 1969-I997 die Abteilung Pädagogik im Erziehungsdepartement des Kantons Solothurn und war in einigen Kommissionen der EDK aktiv, auch in der pädagogischen Kommission.

I3 I K. Frey, 1968b.

I32 Die Arbeitsgruppe (AG) konstituierte sich aus den Mitgliedern der PK, allerdings finden sich auf der Liste der AG keine Delegierten der KOSLO. Dazu exemplarisch Jenzer, Einladung an die Mitglieder der AG, I3. 5. 1976, Archivalien der FAL.

I 33 Konferenz der kantonalen Erziehungsdirektoren, I977, S. 8.

I34 Jenzer et al., 1978, S. 9.

I35 Eugen Egger war 1968-1985 Generalsekretär der Schweizerischen Konferenz der kantonalen Erziehungsdirektoren (EDK). 1962-1983 war er zudem Direktor der Schweize- 
Übergreifend zu den vier Kapiteln des Jahrbuches stehen die beiden Fragen: «Was können wir tun, um unsere kantonalen Lehrpläne zu verbessern? Was können wir tun, um allzu grosse oder unerwünschte Unterschiede in der Schulbildung der fünfundzwanzig Kantone abzubauen?» ${ }^{136}$ Die vorliegende Problemstellung sei aber nicht nur wissenschaftlich erörtert worden, zumal die beteiligten Autorinnen und Autoren allesamt eine «Scharnierstellung zwischen Forschung, Schulpraxis und Schulpolitik einnehmen» und aus der Sicht des «schulpolitisch engagierten Erziehungswissenschaftlers» schreiben würden. ${ }^{137}$ Besonders deutlich wird diese Kombination aus wissenschaftlicher und politischer Haltung am Ende zweier Kapitel, welche mit konkreten Vorschlägen für weitere Schritte abschliessen und als Resultate der Tagung von 1976 angekündigt werden. Dabei handelt es sich um Buchteile, welche erstens Funktion, Form und Inhalt künftiger Lehrpläne abhandeln und zweitens die Verfahren der Entwicklung künftiger Lehrpläne erörtern. Sie werden nun kurz erläutert. Die Vorschläge zu Funktion, Form und Inhalt künftiger Lehrpläne argumentieren in aller Deutlichkeit für fünf Elemente, welche in künftigen Lehrplänen formuliert werden sollten: Leitideen, Unterrichtsziele, ein Stoffverteilungsplan, methodisch-didaktische Hinweise sowie Literatur, Hilfsmittel und Medien. Erst wenn diese fünf Elemente Berücksichtigung fänden, werde der Lehrplan zum koordinationstauglichen Instrument, insbesondere weil Lehrplanvergleiche dann einfacher seien, so eines der Hauptargumente. ${ }^{138}$

Das Teilkapitel wird von der Grafik 4 abgeschlossen und präsentiert die hierarchische Gliederung der als lehrplanrelevant gesetzten Elemente. Eine Sammlung von generellen, einzelne Fachbereiche begründenden und erklärenden Aussagen, in der Grafik «Leitideen» genannt, gilt als Grundlage für die Formulierung von Zielen auf unterschiedlichen Abstraktionsebenen. Die Ziele sollten die zu vermittelnden Einstellungen der Schülerinnen und Schüler umfassen, welche wiederum zu Lerninhalten und Anforderungen an den Unterricht führen würden. Erst der Stoffverteilungsplan nennt gemäss diesem Modell konkrete, überprüfbare schulische Inhalte. Dieses knapp beschriebene Schema zur Lehrplanung sollte die Entwicklung künftiger Lehrpläne massgeblich prägen. ${ }^{139}$ Das Kapitel vier des Jahrbuches der EDK von 1978 präsentiert Vorschläge zu Verfahren der Entwicklung künftiger Lehrpläne und bezieht sich ebenfalls explizit die auf Debatten an der Tagung der PK von 1976. Es enthält konkrete

rischen Dokumentationsstelle für Bildungsfragen (CESDOC) und ab 1968 Professor für Erziehungswissenschaft an der Universität Genf.

I 36 Jenzer und Trier, I978, S. I I.

I 37 Ebd.

I 38 Stricker, Isenegger und Santini, I978b.

I39 Vgl. das Folgekapitel. 


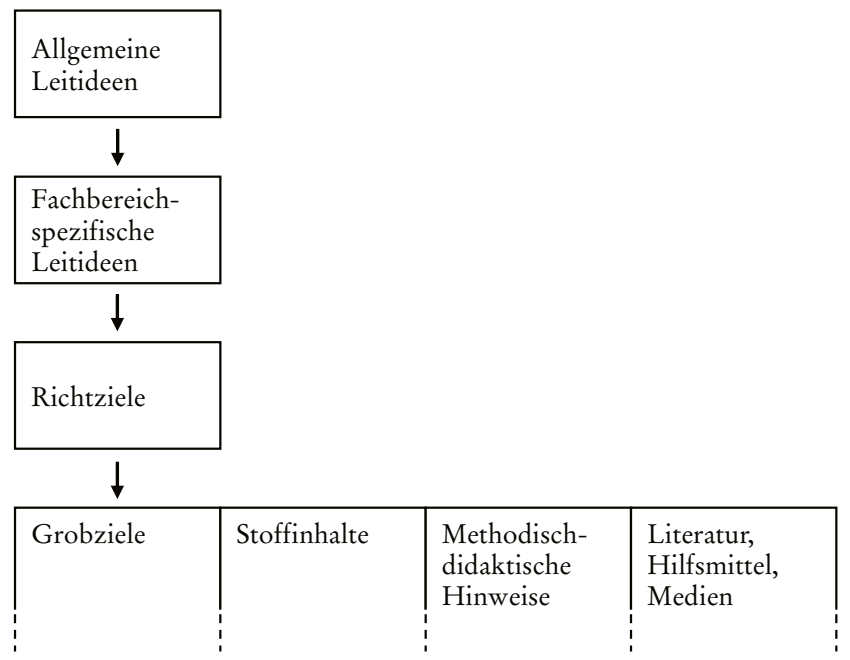

Quelle: Vorschläge betreffend Minimalanforderungen an die Form von Lehrplänen, Jenzer et al., 1978, S. 87

Aussagen zur Planung einzelner Phasen in der Lehrplanentwicklung, schlägt die Installierung eines «Informationssystems» vor, damit alle am Projekt Beteiligten angemessen informiert sind, oder empfiehlt «besondere Sorgfalt» bei der Abklärung von Rahmenbedingungen für das Projekt. ${ }^{10}$ Weiter umfassen die Vorschläge Anregungen zur Einbindung der Berufsverbände und der Elternorganisationen in den Entwicklungsprozess von Leitideen und darüber hinaus folgender Akteure: «Neben der breiten Beteiligung der Lehrkräfte bei der Erarbeitung eines Lehrplanes ist für den ganzen Entwicklungsprozess auch die Mitwirkung von Fachleuten der Bildungswissenschaft und der für die Inhalte der einzelnen Lernbereiche kompetenten Fachleute notwendig.» ${ }^{\mathrm{I}}$ Speziell erwähnt wird der erforderliche Beizug kompetenter Wissenschaftler und der Einsatz von Beratungsdiensten. «Diese stellen die Koordination zwischen den beteiligten Einzelwissenschaftern, die Rekrutierung zusätzlicher Wissenschafter oder Fachleute sowie die Vermittlung universitärer Forschung/ Theoriebildung und Schulpraxis sicher. $\gg^{142}$

I 40 Strittmatter, $1978 \mathrm{~d}$, S. I 20.

I4 I Ebd.

142 Ebd. 
Letztlich publizierte die EDK im Jahrbuch von 1978 den aktuellen Stand ihrer Diskussionen. Sie bündelte damit die Entwicklungslinien der kantonalen Lehrplanpolitik bis in die frühen r990er-Jahre. ${ }^{143}$ Sie schuf ferner Anknüpfungspunkte und Orientierung für kantonale und interkantonale Reformprojekte mit Lehrplanbezügen, und eines davon verantwortete sie unter dem Namen «Überprüfung der Situation der Primarschulen» (SIPRI) selbst. ${ }^{\mathrm{I} 4}$

Die Situation der Primarschulen sollte auf Druck der Lehrerverbände überprüft werden, nachdem die EDK 1975 den Kantonen empfohlen hatte, den Unterricht in der zweiten Landessprache in die Primarschule vorzuverlegen. Das bisher grösste gesamtschweizerische Reform- und Forschungsprojekt unter der Leitung der EDK dauerte von 1978 bis 1986 und war in vier Teilprojekte gegliedert: I. Lernziele der Primarschule - Anspruch und Wirklichkeit, 2. Funktionen und Formen der Schülerbeurteilung, 3. Übergang von der Vorschulzeit in die Primarschule, 4. Kontakte und Zusammenarbeit zwischen Schule und Elternhaus. ${ }^{145} \mathrm{Im}$ Rahmen des ersten Teilprojektes wurden etliche Entwicklungskataloge für Lehrplanrevisionen verfasst, Beratungen bei laufenden Lehrplanrevisionen durchgeführt, Basisinitiativen an unzähligen Schulen begleitet, entsprechende Informationsmaterialien für die Lehrerinnen- und Lehrerbildung entwickelt, regionale Gesprächsrunden organisiert oder bestehende Leitideen in kantonalen Lehrplänen verglichen und idealtypische «SIPRI-Leitideen» entwickelt. ${ }^{146}$ Diese nicht abschliessende Aufzählung von Tätigkeiten zeigt in aller Deutlichkeit die thematische wie disziplinäre Breite, mit welcher gesamtschweizerisch während acht Jahren Lehrplanfragen debattiert und bearbeitet wurden. Entsprechend waren Wissenschaftler, Lehrpersonen, Seminarlehrerinnen und -lehrer wie auch Personen aus den kantonalen Planungs- und Verwaltungsstellen ins Projekt involviert.

Ein Austausch zwischen Wissenschaft, Politik, Praxis und Verwaltung in Bezug auf Lehrplanfragen fand auch auf internationalem Parkett statt. Zum Thema «Bedingungen und Modelle der Curriculuminnovation» nahmen vom 28. September bis I I. Oktober 1975 in Schlierensee (D) über Ioo Personen aus Bildungsverwaltung, Bildungsforschung, Fachdidaktik und Schulpraxis an einem internationalen Seminar teil, wobei knapp ein Fünftel der Teilnehmenden aus der Schweiz angereist war. Das Ziel bestand darin, Grundlagen «für Aus- und Fortbildungsveranstaltungen sowie zur Lösung von Aufgaben im Bereich der Forschung, Bildungsplanung, Projektorganisation und Bildungspolitik» aus-

I 44 SIPRI wird im Folgekapitel ausführlicher behandelt, die Ausführungen hier sind entsprechend kurz gehalten.

I45 J. P. Meylan, I979.

I46 Strittmatter, I I. 6. I982, StALU, Ai $270 / 956$. 
zuarbeiten. ${ }^{147}$ Die Veranstaltung wurde organisiert vom Institut für die Pädagogik der Naturwissenschaften (IPN) der Universität Kiel in Zusammenarbeit mit dem Center for Educational Research and Innovation (CERI) der OECD. Basierend auf den bisherigen Erfahrungen mit Projekten, die auf die Konstruktion von neuen Curricula abzielten, hatte sich die Debatte mittlerweile unter Berücksichtigung von organisationssoziologischen Ansätzen auf die Frage konzentriert, «wie ein neues Curriculum zu einem wirksamen Bestandteil der Arbeit und des Lebens von Schulen werden kann». ${ }^{148}$ Die Curriculumforscher aus der Schweiz stützten sich in ihren Ausführungen weitgehend auf Berichte aus laufenden Curriculumentwicklungsprojekten. So thematisierten Karl Frey und Bruno Santini «die Legitimation von Innovationszielen im Innovationsprojekt Weiterbildungsschule Zug», während Joseph Eigenmann zur «Bestimmung und Behebung von Konflikten und Konfliktfeldern» anlässlich eines Curriculumprojektes im Kanton Freiburg referierte. Heinz Moser präsentierte sein Projekt über die «Innovationsstrategische Konzipierung von Projekten im Sinne handlungsorientierter Curriculumforschung». ${ }^{149}$

Aus der Perspektive der Bildungsverwaltung präsentierten Uri Peter Trier ${ }^{150}$ und Walo Hutmacher ${ }^{151}$ die Hintergründe und Argumentationslinien der leitenden Fragestellung der Tagung. Unter dem Titel «Zusammenarbeit zwischen Forschung, Schulpraxis, Schulverwaltung und Politik. Problemstellungen, Modelle, Empfehlungen für künftige Arbeiten» gingen die beiden Referenten und Autoren des gleichnamigen Artikels im Tagungsband des Seminars den Voraussetzungen der gewünschten Zusammenarbeit nach. Das Nachdenken über schulische Lerninhalte, deren Auswahl oder didaktische Aufbereitung im Unterricht habe aufgezeigt, dass dazu keine wissenschaftlichen Antworten möglich und darum politische Antworten erforderlich seien. Aus diesem Grund sei die curriculare Aufgabe nur in «Zusammenarbeit (z. B. bei Curriculumentwicklungsprojekten) unter Berücksichtigung der gegenseitigen Interessenlage» von allen beteiligten Akteuren zu leisten. ${ }^{52}$ Dazu zählten ihrer Ansicht nach die Felder Politik, Forschung, Verwaltung und Schulpraxis, die je unterschied-

I47 Hameyer, Aregger und Frey, 1976, S. 2.

I48 Waterkamp, I976, S. 333.

I49 Hameyer et al., I976.

I 50 Uri Peter Trier studierte Psychologie und Soziologie in Zürich und Basel, bevor er in der akademischen Berufsberatung tätig wurde. 1971-1990 leitete er die pädagogische Abteilung der Erziehungsdirektion des Kantons Zürich. Er präsidierte in den I970er-Jahren die pädagogische Kommission der EDK.

I I Walo Hutmacher studierte Soziologie und gründete nach seiner bildungsplanerischen Tätigkeit für die UNESCO I965 den Service de la recherche sociologique, welchen er bis 1995 leitete. Daneben lehrte er an den Universitäten Genf, Zürich und Neuenburg Bildungssoziologie.

I52 Hutmacher und Trier, 1976, S. I0I. 
lich miteinander in Beziehung stünden. Wenn letztlich der Erziehungsprozess effizienter gestaltet werden solle, sei das «von oben gesteuerte Industriemodell der Forschungsorganisation in Frage» zu stellen. ${ }^{153}$ Stattdessen müssten «andere Formen der Zusammenarbeit zwischen Forschung und Praxis, sowie neue Formen der Eingliederung und Steuerung der Forschung in der Verwaltung gesucht werden». ${ }^{54}$ Die beiden Autoren votierten demgemäss für eine stärkere Forschungsorientierung und Übernahme von Projektverantwortung in der Verwaltung.

\subsubsection{Umtriebige Vortragstätigkeit der FAL}

Für die I970er-Jahre insgesamt ist ein breites Interesse an Informationen zu verschiedenen Facetten aus dem Bereich der Curriculumforschung zu konstatieren. Erläuterungen waren beispielsweise dazu erwünscht, weshalb eine «Lehrplanreform als Grundlage der Schulreform» angezeigt sei, welche «Möglichkeiten in einem Entwicklungsprojekt» stecken, welche «Techniken zur Lernzielformulierung» bestehen oder wie die «Operationalisierung von Lernzielen» anzugehen sei. ${ }^{\text {Iss }}$

Der Bedarf an spezifischen Informationen zu Curriculumreformen ist nicht nur in der während knapp zehn Jahren beachtenswerten Dichte von Artikeln in einschlägigen Zeitschriften für Lehrerinnen und Lehrer abzulesen. ${ }^{156}$ In den Jahresberichten der Freiburger Arbeitsgruppe für Lehrplanforschung liegt ein deutlicher Nachweis für die ausserordentlich intensive Referatstätigkeit ihrer Mitglieder aufgelistet vor. Diese Angaben dienen im Folgenden für die Illustration dafür, dass die FAL ausserhalb der akademischen Community und insbesondere direkt in der Aus- und Weiterbildung der Lehrerinnen und Lehrer für ihre Position und ihre Argumente zugunsten einer curriculumbasierten Bildungsreform warben.

Die Tabelle 2 zeigt die Zahl der in den Jahren 1971-1978 von Mitgliedern der Freiburger Arbeitsgruppe gehaltenen Referate.

I 53

I 54

I 55

I 56

Ebd., S. II 5 .

Ebd.

Forschungszentrum FAL, Jahresbericht 1971, Archivalien der FAL, S. i f f.

Eine Analyse der Zeitschriften für Lehrerinnen und Lehrer, insbesondere der «Schweizer Schule» und der «Schweizerischen Lehrerzeitung», zeigt die beachtliche Publikationsdichte an Artikeln im Curriculumbereich ab den frühen I970er-Jahren, welche bis 1980 sukzessive abnimmt, um dann ganz zu verschwinden. Dazu weiterführend Höhener, 2018. 


\begin{tabular}{lrrrr} 
Jahr & $\begin{array}{r}\text { wissenschafticher } \\
\text { Anlass }\end{array}$ & $\begin{array}{r}\text { nichtwissenschaft } \\
\text { licher Anlass }\end{array}$ & $\begin{array}{r}\text { Total } \\
\text { Referate }\end{array}$ & $\begin{array}{r}\text { davon Anlass } \\
\text { der LehrerInnen- } \\
\text { bildung }\end{array}$ \\
\hline 1971 & 2 & 18 & 20 & 11 \\
1972 & 3 & 17 & 20 & 6 \\
1973 & 3 & 35 & 38 & 10 \\
1974 & 3 & 23 & 26 & 12 \\
1975 & 1 & 12 & 13 & 5 \\
1976 & 1 & 17 & 18 & 10 \\
1977 & 0 & 7 & 7 & 7 \\
1978 & 1 & 10 & 11 & 5 \\
\hline Total & 14 & 139 & 153 & 66
\end{tabular}

Gezählt wurden die Anlässe, an denen ein Referat eines FAL-Mitglieds gehalten wurde. Als ein Anlass gelten auch die vielen mehrtägigen Ereignisse wie Weiterbildungswochen oder Vorlesungen an Lehrerseminaren.

Quelle: Eigene Darstellung

Die Redeanlässe werden zunächst aufgeschlüsselt nach Anlässen mit vorrangig wissenschaftlichem oder nichtwissenschaftlichem Publikum. Den Angaben in der Spalte «wissenschaftlicher Anlass» ist zu entnehmen, dass die Mitglieder der FAL primär in der ersten Hälfte der I970er-Jahre auf wissenschaftlichen Kongressen zu Themen aus ihrer Curriculumforschung referierten. ${ }^{157}$ Gemessen an der gesamten jährlichen Referatstätigkeit sind die genannten Zahlen tendenziell tief, zumal grosse wissenschaftliche Kongresse in den I970er-Jahren noch nicht häufig waren.

Die Tabelle 2 zeigt auf, dass die Mitglieder der FAL knapp zehnmal häufiger vor wissenschaftsfernen Zuhörenden referierten als etwa an wissenschaftli-

I57 Auch anhand der hier vorliegenden Zahlen wird die These aus dem Kapitel zwei gestützt, wonach ab Mitte der 1970er-Jahre zunehmend weniger Forschende im Themenbereich Curriculumforschung tätig waren. 
chen Kongressen. Sie sprachen zu Lehrpersonen im Rahmen von Fortbildungen, hielten Vorträge vor Schülerinnen und Schülern, bei Elternvereinigungen oder Vereinsanlässen, referierten an Parteianlässen oder vor Vertreterinnen und Vertretern kantonaler «Kader» des Bildungswesens. ${ }^{158}$ Thematisch lassen sich diese Referate nur bedingt systematisieren. Am Beispiel des Berichtes von I $973^{159}$ wird im Folgenden ein Versuch unternommen: Die genannten Referatstitel versprechen erstens Informationen über «Problemstellungen und Ziele in der Curriculumforschung» oder zu «Funktionen des Curriculum». Diese und ähnliche Referate verfolgten offenbar das Ziel, über die eigene wissenschaftliche Forschungstätigkeit aufzuklären. Ein zweiter Themencluster könnte als Versuch gewertet werden, aktuelle bildungspolitische Reformvorhaben zu erläutern und einzuordnen. Demgemäss lauteten die Referatstitel etwa «Die Leitideen der Schulreform in der Zentralschweiz» oder «Bildungspolitik als Gesellschaftspolitik». Ein drittes Themenfeld wurde durch explizit lehrberufsspezifische Referate abgesteckt: «Vom Heimatkundeunterricht zum Sachunterricht», «Unterrichtsvorbereitung», «Tendenzen in der Reform der gymnasialen Unterstufe» oder «Lehrer und Curriculum». Schliesslich finden sich vereinzelt Referatstitel, die kaum Bezüge zum Forschungsgegenstand der FAL aufweisen.

\subsubsection{Curricula als Dreh- und Angelpunkt}

Curricula sind in ausserwissenschaftlichen Debatten der 1970er-Jahre zu einem Vektor mit unterschiedlichen Funktionen geworden. Sie bereiteten das Feld für Diskussionen über das Berufsbild, die Ausbildungsinhalte und die zu erwerbenden Qualifikationen künftiger Lehrpersonen. Sie avancierten zum Koordinationswerkzeug der EDK, die wiederum freimütig Form, Funktionen und Inhalte künftiger Lehrpläne und auch die Entwicklungsverfahren empfahl. Letztlich wurden Curricula aber auch zum Innovationswerkzeug und zum gestaltenden Element der Schule erhoben.

Am Anfang der Auseinandersetzungen, sei es über die Fixierung von Inhalten für die künftige Lehrerinnen- und Lehrerbildung oder über einzuleitende Entwicklungen in der Volksschule, formulierten die Beteiligten an Konferenzen und in Projekten stets Kritik am Bestehenden: Die derzeitige Ausbildung sei generell mangelhaft, sie werde den jüngsten Ansprüchen nicht gerecht. Die Curricula der Volksschule seien inhaltlich überfrachtet und für die Lehrpersonen im Berufsalltag wenig unterstützend. Oder: Die Unterschiede zwischen

I 58 Forschungszentrum FAL, Tätigkeitsbericht 1974, S. I 5 , Archivalien der FAL.

I59 Forschungszentrum FAL, Tätigkeitsbericht 1973, Archivalien der FAL. 
den kantonalen Curricula seien frappant und seien zu beheben. Dieselben Argumente, welche von den Promotoren der Curriculumforschung bereits zur Legitimation umfassender Reformen vorgebracht worden waren, sind in bildungspolitischen Debatten rezipiert worden.

Die politischen Prozesse zur Bearbeitung der formulierten Problemlagen verlangten, dass Akteure aus der Politik, der Wissenschaft, der Schulpraxis und der Bildungsverwaltung im Rahmen von Projekten, Kommissionen und Tagungen in Austausch kamen und es je nach Geschäft für längere Zeit bleiben sollten. Sowohl die Debatten in der pädagogischen Kommission der EDK als auch jene anlässlich der Reformbemühungen der Lehrerinnen- und Lehrerbildung haben gezeigt, dass vonseiten der Wissenschaft wohl Idealvorstellungen dargelegt wurden, diese aber weder in sich kongruent noch politisch telquel umsetzbar waren. Für die Mitglieder der FAL war das kein Hinderungsgrund, ein umtriebiges Referatswesen zu betreiben und ihr Ziel der Bekanntmachung der Curriculumforschung in einer breiten Öffentlichkeit und in der Lehrerinnen- und Lehrerbildung zu verfolgen.

Curricula wurden in den Debatten zu bildungspolitischen Reformen als Drehund Angelpunkt der jeweiligen Vorhaben konzipiert, wobei dieses Vorgehen nach weiteren, mit in die Debatten einzubringenden Expertisen verlangte. So gaben der Schweizerische Pädagogische Verband oder die EDK Forschungsarbeiten in Auftrag, welche dann Wissenschaftler wie die Mitglieder der FAL erstellten.

Dass die hier analysierten Debatten über die scheinbar notwendigen Elemente oder einzubeziehenden Inhalte bei der Konstruktion von Curricula nicht allein unter Curriculumforschenden, sondern in politischen Zusammenhängen geführt wurden, wird auch an einer begrifflichen Nuance ersichtlich: Bereits in den Anfängen der Curriculumforschung verwendeten Forschende zur Bezeichnung ihres Bereiches den Begriff Curriculum. Besonders im Teilkapitel zu Austausch zwischen Schulpraxis, Bildungsplanung und -forschung zeigen die angeführten Quellen und Zitate aber ein offensichtliches Ignorieren dieses Begriffs. Insbesondere in Debatten der EDK wurde konsequent das Wort «Lehrplan» verwendet. Man hatte sich zwar auf Konzepte und Strategien jener Forschungsrichtung eingelassen, der herkömmliche Begriff blieb aber unverändert. 


\subsection{Wissenschaftler und Experten in Wissensnetzwerken}

Dieses Kapitel hat ganz zu Beginn die Frage aufgeworfen, wie es dazu kam, dass Curricula als zunächst spezifischer Gegenstand der Forschung zum Thema umfangreicher und jahrelanger bildungspolitischer Debatten avanciert sind. Eine ähnliche Frage hat Armin Gretler für die Themensetzung innerhalb der schweizerischen Bildungsforschung gestellt und sogleich auf institutionelle Strukturen verwiesen. ${ }^{60}$ Die einzelnen Institute und Professuren seien es, die in der Forschung die Themen setzen würden. Ebenfalls nicht zu vergessen seien die verwaltungsinternen pädagogischen Planungs- und Arbeitsstellen, welche ihre Arbeiten an den Bedürfnissen ihrer Träger, der kantonalen Erziehungsdepartemente, ausrichten würden. Auch der Nationalfonds habe Anteil an der Themensetzung in der Forschung mit dem Format der nationalen Forschungsprogramme. Diese richten sich nach gesellschaftlichen Erwartungen und Bedürfnissen aus.

Im hier gegebenen Fall gestaltet sich die Problemstellung komplexer. Nicht die Setzung eines Themas innerhalb eines ausgewählten gesellschaftlichen Feldes wie der Wissenschaft, ${ }^{161}$ sondern dessen Rezeption darüber hinaus in bildungspolitischen Debatten ist zu klären. Um den konstatierten Austausch von Wissensbeständen über gesellschaftliche Felder hinweg fassbar zu machen und mögliche Erklärungen dafür zu generieren, wird im Folgenden auf die Resultate einer historischen Netzwerkanalyse zurückgegriffen.

Ein netzwerkanalytischer Zugriff stellt grundsätzlich die Akteure und ihre Beziehungen in den Vordergrund. ${ }^{162}$ Übertragen auf die vorliegende Problemstellung wird davon ausgegangen, dass Delegierte in Kommissionen oder an Konferenzen Referierende als Träger bestimmter Informationen und Wissensbestände zu begreifen sind und demgemäss ihre Ansichten in die Debatten als Zugehörige einer spezifischen «epistemic community» (EC) einbringen. ${ }^{63} \mathrm{Da}$ bei handle es sich um eine Möglichkeit, eigene Anliegen und Interessen einzubringen und besonders in politischen Kontexten Deutungshoheiten abzustecken und die Verteilung von Ressourcen zu eigenen Gunsten zu beeinflussen. ${ }^{164}$ Eine eigenartige Stellungnahme zur Zusammensetzung eines Gremiums hat Hans Gehrig einleitend in den Tagungsband der Konferenz zu «pädagogischen Disziplinen in der Lehrerbildung» ${ }^{165}$ von 1970 einfliessen lassen: «Mit

I60 Gretler, 2000.

I6I Bourdieu, I997.

I62 Düring, Eumann, Stark und Keyserlingk, 2016.

I63 Haas, 200I; 20I I; Knorr Cetina, I999.

I64 Szöllösi-Janze, 2004.

I65 Gehrig, I970a. 
der Einführung der Experten wird die Personabhängigkeit in den Planungsprozess eingeführt, jedoch - wie uns scheint - objektiviert durch die Tatsache, dass die Fachleute nur für ihr eng und genau begrenztes Gebiet [...] Aussagen liefern.» ${ }^{166}$ Gehrig als Beteiligter und Vertreter eigener Interessen im Bereich der Lehrerinnen- und Lehrerbildung schien es offenbar angezeigt, das Gewicht und die Selektivität der in Gremien involvierten Expertinnen und Experten zu relativieren.

Die Netzwerkanalyse basiert auf der systematischen Erhebung von Personendaten in den Quellenmaterialien aller in diesem und im Vorkapitel beschriebenen Konferenzen, Seminare und Projekte, welche sich mit curricularen Fragen auseinandergesetzt haben. Anhand der Listen der Teilnehmenden und Referierenden wurde dann das Feld der Debatten rekonstruiert. Anschliessend wurden die Daten ergänzt mit Angaben zu institutionellen Verortungen wie Studienorten oder Arbeitsorten der Personen ${ }^{167}$ um individuelle Berufsbiografien zu rekonstruieren wie auch eine institutionelle Verortung der Personen in gesellschaftlichen Feldern vorzunehmen.

Zur Sammlung und Aufbereitung des umfangreichen Datenmaterials wurde auf Nodegoat ${ }^{168}$ zurückgegriffen. Es handelt sich dabei um eine webbasierte Datenmanagementumgebung. Sie ist insbesondere für Netzwerkanalysen und Visualisierungen für historische Forschungsprojekte programmiert worden und erlaubt jene Flexibilität in der Definition von beziehungsrelevanten Analysekategorien, welche im Umgang mit dem Quellenmaterial unabdingbar ist.

\subsubsection{Verflechtung und Dominanz der FAL in den Debatten}

Das Ziel der Grafik s besteht darin, das Feld der Teilnehmenden in Projektgruppen zu visualisieren, allerdings zunächst mit einer Einschränkung: Es werden zwar sämtliche in diesem Kapitel präsentierten Projektgruppen als schwarze Punkte dargestellt. Von den in den Gruppen vertretenen Personen werden aber vorerst nur die Mitglieder der Freiburger Arbeitsgruppe abgebildet.

Beat Mayer engagierte sich beispielsweise im Projekt «Schulkoordination über Lehrplanreform» der EDK. Er ist wie jede andere Person als weisser Punkt dargestellt und mit dem entsprechenden Projekt verbunden. Es finden sich auch

I66 Möller, 1969, S. 79 f.

I67 Die zusätzlichen Personendaten wurden ausschliesslich aus öffentlich einsehbaren Quellen wie etwa dem «Historischen Lexikon der Schweiz» und anderen auffindbaren Nachweisen wie Nachrufen gewonnen oder Autorenverzeichnissen in Publikationen entnommen.

https://nodegoat.net. 


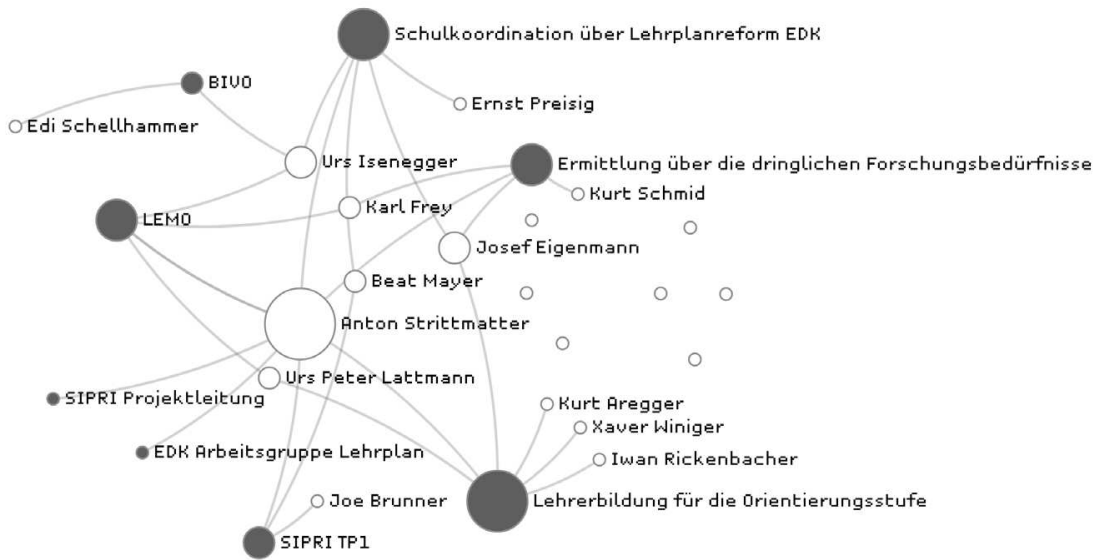

Quelle: eigene Darstellung

Mitglieder der FAL, welche in keiner Projektgruppe aktiv waren. Sie sind als weisse Punkte ohne Verbindung zu Projekten dargestellt. Im Vergleich zum Abbild von Beat Mayer ist der weisse Punkt von Anton Strittmatter deutlich grösser. Damit wird verdeutlicht, dass Strittmatter in mehreren Projekten aktiv war und er dementsprechend mehr Verbindungen aufweist. Generell ist in der Grafik s zu erkennen, dass die Mitglieder der FAL in den Projektgruppen sehr aktiv waren, in jedem Projekt war mindestens eine Person der FAL beteiligt. Anton Strittmatter engagierte sich ausser in BIVO sogar in allen Projekten. Bloss sieben der insgesamt 20 Mitglieder der FAL haben sich nicht in Projekten engagiert, in welchen Curriculumdebatten geführt wurden.

Die Grafik 6 zeigt wiederum sämtliche Projektgruppen zu Curriculumdebatten der 1970er-Jahren. Im Unterschied zur Grafik 5 wird hier aber das Gesamtnetzwerk der Projektmitarbeitenden abgebildet. Während vorhin nur die Mitglieder der FAL als weisse Punkte abgebildet wurden, zeigt das Netzwerk in der Grafik 6 sämtliche an den Projekten teilnehmenden Personen und ihre Verbindungen mit denjenigen Projekten, in welchen sie aktiv waren.

Der Blick auf das Gesamtnetzwerk der Projekte verdeutlicht insbesondere zwei Dinge. Erstens zeigen beispielhaft die Projekte LEMO und Lehrerbildung für die Orientierungsstufe in der Zentralschweiz, dass unter den Projektteilnehmenden in der Regel zwei Gruppen von Mitgliedern auszumachen sind. Die anzahlmässig grössere Gruppe der Projektinvolvierten war ausschliesslich 


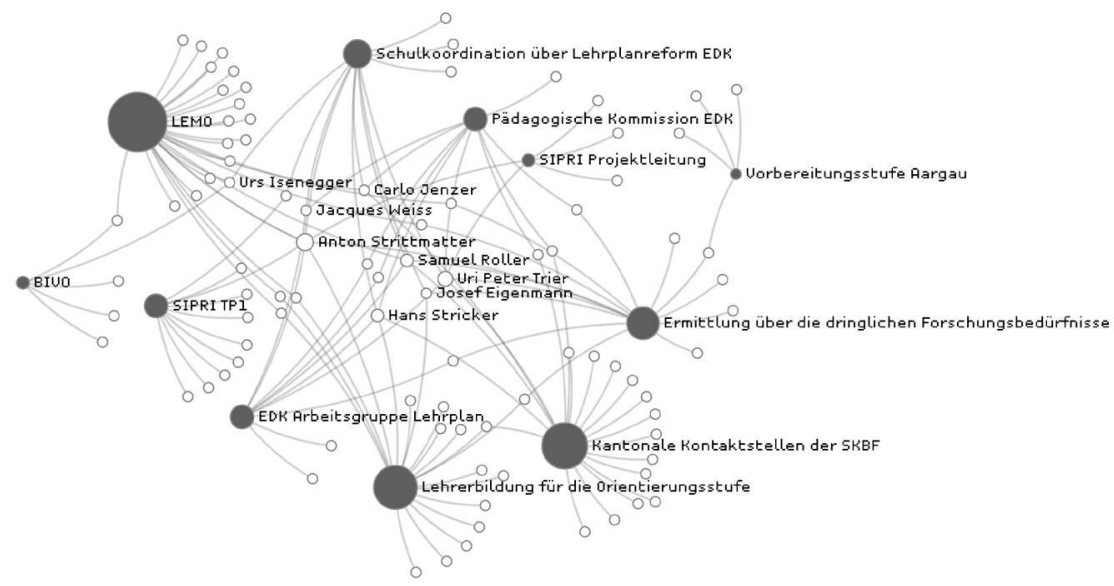

Quelle: eigene Darstellung

in einem Projekt engagiert. Sie weisen nur eine Verbindung auf und sind in der Grafik 6 kreisförmig um jenes eine Projekt angeordnet. Daneben waren in den Projekten jeweils einige wenige Personen aktiv, welche in mehreren Projekten engagiert waren. Diese Personen sind in der Mitte der Grafik positioniert. Ab vier Verbindungen ist ihr Name eingeblendet. In der Literatur zu Netzwerkanalysen werden diese Personen als «Broker» bezeichnet. ${ }^{169}$ Sie haben eine Schlüsselposition inne und verbinden Strategien, Erfahrungen und Wissensformen über verschiedene Projekte hinweg. Von den hier abgebildeten Schlüsselpositionen wurden drei von Mitgliedern der FAL eingenommen.

Die Grafik 6 verdeutlicht zweitens, dass die Schlüsselpositionen in projektbezogenen Curriculumdebatten oft von Personal aus Bildungsplanungsstellen und pädagogischen Arbeitsstellen eingenommen wurden. Mit Uri Peter Trier, Hans Stricker, Carlo Jenzer, Samuel Roller und Jacques Weiss waren entsprechende Stellen aus Zürich, Bern, Solothurn und der Romandie vertreten. In dieser Gruppe mitzuzählen ist Anton Strittmatter, welcher nach seiner Tätigkeit bei der FAL die Leitung des Zentralschweizer Beratungsdienstes übernommen hat.

Letztlich bedeutet die Verteilung der Schlüsselpositionen auf Planungs- und Verwaltungspersonal sowie auf die Mitglieder der FAL auch, dass kaum wei-

I69 Düring und Keyserlingk, 20 I 5; Fangerau, 2009; Nagel, 2009. 


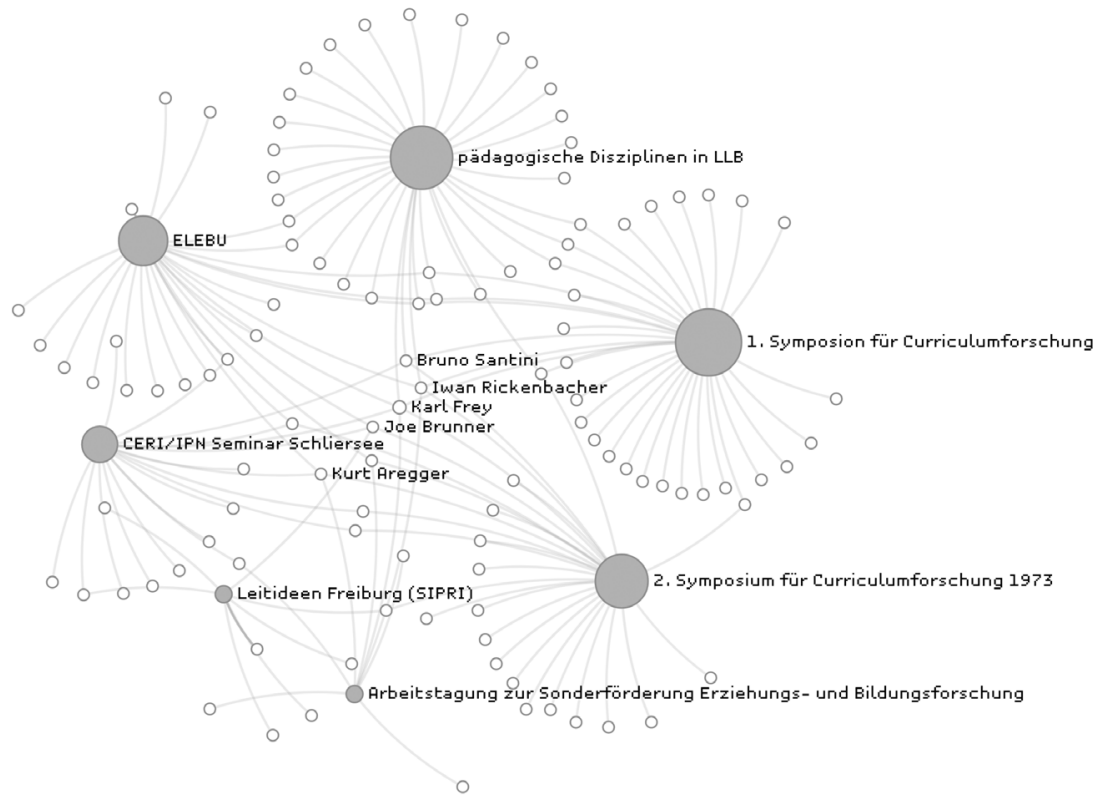

Quelle: eigene Darstellung

tere Exponenten aus der Wissenschaft in den zu führenden Debatten vertreten waren, sodass sie sich mit Nachdruck hätten einbringen können. Gestaltungsfragen im Volksschulbereich oder die Neuausrichtung der Lehrerinnen- und Lehrerbildung zog an den Wissenschaftsvertreterinnen und -vertretern etwa der Universitäten Bern, Zürich, Genf oder Basel weitgehend vorbei.

Die Grafik 7 visualisiert die an Konferenzen und Seminaren Teilnehmenden. Anders als bei den oben diskutierten Projekten handelt es sich bei den nun thematisierten Anlässen um Ereignisse, welche deutlich weniger zeitintensiv waren und sich in der Regel auf zwei bis drei Tage beschränkten. Die Grafik zeigt anschaulich, dass sich die Teilnehmenden in der überwiegenden Mehrheit nur einmal in die Debatten einer Konferenz beziehungsweise eines Seminars eingeschaltet haben. ${ }^{170}$ Diejenigen Akteure aber, welche an Konferenzen und Seminaren am besten vernetzt waren, gehörten ausnahmslos zur Freiburger

I70 Es ist an dieser Stelle zu vermerken, dass die Konferenzen und Seminare bisweilen über 80 Teilnehmende verzeichneten. Ausgehend von der Überlegung, dass hauptsächlich die Referierenden mit ihren Beiträgen die Debatten zu prägen vermochten, wurden nur diese in die Analysen einbezogen. 

1970er-Jahre

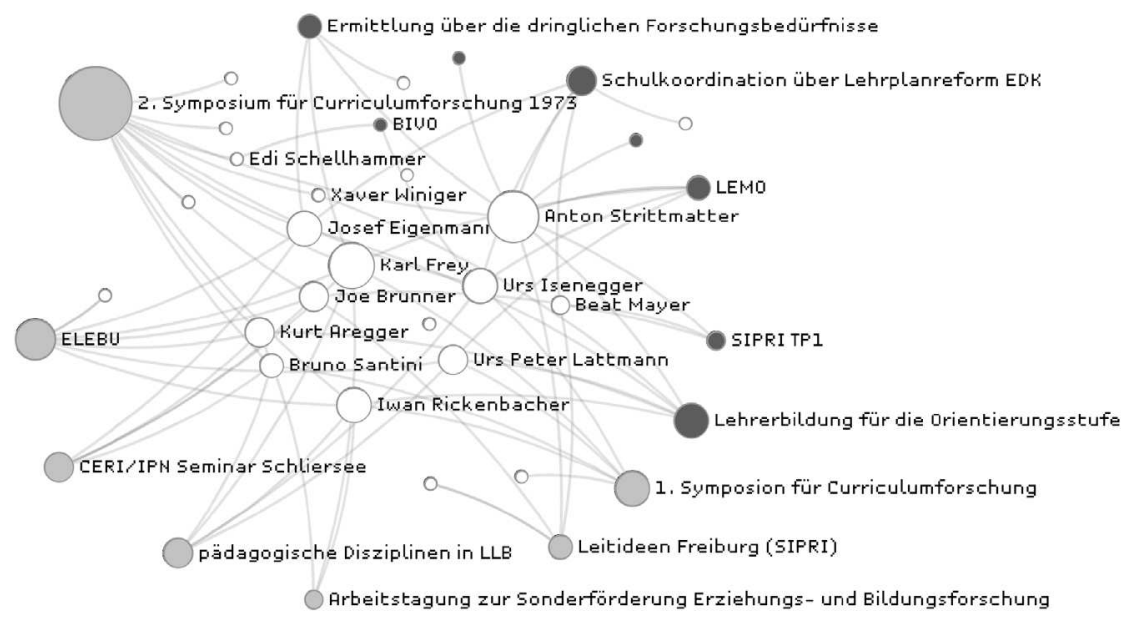

Quelle: eigene Darstellung

Arbeitsgruppe. Karl Frey war bis auf eine Konferenz anlässlich von SIPRI im Kanton Freiburg überall referierend vertreten. ${ }^{17 \mathrm{I}}$

Aufgrund der bisherigen Ausführungen ist festzuhalten: Es handelt sich um etwa ein Dutzend Personen, welche die Schlüsselpositionen in den Debatten auf Konferenzen und Seminaren wie auch anlässlich von Projekten im Analysezeitraum von zehn Jahren eingenommen haben. In diesen Debatten haben die Mitglieder der FAL prominent mehrere Schlüsselpositionen eingenommen. Was sie von allen anderen Personen in den Gremien und an Konferenzen unterscheidet, ist die Tatsache, dass sie sowohl der universitären Curriculumforschung angehörten als auch in die bildungspolitische Projektarbeit und die damit verbundenen Konferenzen involviert waren. Diese direkte personelle Verflechtung von Forschung und Entwicklung pädagogischen Wissens mit bildungspolitischer Projektarbeit macht die Grafik 8 nochmals deutlich.

In der Grafik werden die Projektgruppen und die Konferenzen gemeinsam abgebildet. In direkter Verbindung stehen sie durch die Mitglieder der FAL, wel-

I7 I Dass Karl Frey in vergleichsweise wenigen Projekten engagiert war, aber kaum eine Konferenz oder ein Seminar ausgelassen hat, dürfte mit dem Zeitaufwand für die jeweilige Veranstaltungsart zu erklären sein. Da Frey ab 197I in Kiel als ordentlicher Professor forschte, musste er auf zeitintensive Projektarbeiten in der Schweiz verzichten. 
che sich in die entsprechenden Debatten eingebracht haben. Es wird ersichtlich, dass in jeden Diskussionsanlass mindestens ein FAL-Mitglied involviert war. Es zeigt sich aber auch, dass von den 20 Mitgliedern der FAL einige die Debatten äusserst aktiv mitgestalteten.

Zudem geht aus der Grafik deutlich hervor, dass dieselben Personen, die wissenschaftliche Symposien zur Curriculumforschung organisierten und dort referierten, ihre Ideen und Konzepte ebenso in Projektgruppen über die Entwicklung der Lehrplanpolitik in der Schweiz einbringen konnten. Eine Gruppe von Wissenschaftlern bildete, im Jargon der Netzwerkanalyse gesprochen, einen Cluster und besetzte die überwiegende Mehrheit der Debatten mit Bezügen zu Curricula und Bildungsreformen. ${ }^{172}$ Dieser Befund führt zur Eingangsfrage zurück, weshalb Konzepte aus der Curriculumforschung in bildungspolitische Reformdebatten aufgenommen wurden.

\subsubsection{Wissenschaftler und Experten - Wissenschaftler als Experten}

Margrit Szöllösi-Janze ist der Ansicht, dass sowohl die Wissenschaftler zu diskutieren seien, die neues Wissen produzieren, wie auch die Experten, die vorhandenes Wissen reproduzieren, indem sie es einer nachfragenden Klientel aus Politik oder Bürokratie verfügbar machen. ${ }^{173}$ Im vorliegenden Fall liegt die Sache ähnlich und doch anders. Die Mitglieder der Freiburger Arbeitsgruppe sind als universitäre Wissensproduzenten aktiv gewesen, waren gleichzeitig aber auch als Experten in bildungspolitischen Projekten, an ebensolchen Konferenzen und in Planungsgremien vertreten. Wissenschaft und Lehrplanpolitik waren eng verflochten. Die Mitglieder der FAL konnten durch ihre grosse Präsenz in einschlägigen Gremien und an Konferenzen für ihren Wissensbereich Themenkonjunktur betreiben und die Nutzbarmachung ihrer Forschungsresultate erfolgreich voranbringen.

Die Identifikation der einzelnen FAL-Mitglieder mit den Vorstellungen und Grundüberzeugungen der Arbeitsgruppe im Sinne einer «epistemic community» hat massgeblich die breite Rezeption curricularer Konzepte in bildungspolitischen Debatten gefördert. Die Mitglieder verfügten über eine klar formulierte und zielstrebig umgesetzte Strategie der Wissenschaftskommission. Sie agierten koordiniert im Feld bildungsreformerischer Aushandlungen und sie sorgten für die Omnipräsenz ihrer wissenschaftlich legitimierten Positionen,

I72 Düring und Keyserlingk, 2015.

173 Szöllösi-Janze, 2004. 
etwa zur Aufgabenverteilung zwischen Bildungsverwaltung, Schulinspektion und zu involvierenden Experten.

Bei der Auftragsvergabe im Themenbereich der Curriculumforschung, gleich ob es sich um einen Bericht oder um die Begleitung eines Projektes handelte, musste die Wahl auf die Freiburger Arbeitsgruppe fallen. Diese hatte eigens zu diesem Zweck ein internes Merkblatt mit «Vorfragen an potentielle Auftraggeber für Projekte, Gutachten, Beratungen, Kurse» verfasst. ${ }^{174}$ Das Netzwerk der FAL erweiterte sich auf der einen Seite, indem sie ihres Expertenwissens wegen für Aufträge angefragt wurde. Auf der anderen Seite wuchs der Bekanntheitsgrad der FAL, indem ihre Mitglieder zunehmend in hoher Zahl in Gremien vertreten waren und bereits ihre Präsenz als Eigenwerbung fungierte. Die Mitglieder profitierten gewissermassen vom Matthäus-Effekt, wonach Personen mit vielen Beziehungen in Netzwerken mit grösserer Wahrscheinlichkeit neue Beziehungen dazugewinnen. ${ }^{175}$

Das Feld der in Curriculumdebatten involvierten Personen war überschaubar. Anlässlich von zugehörigen Projekten wie auch an einschlägigen Konferenzen und Seminaren traf sich während eines Jahrzehnts immerfort ein kleiner, überschaubarer Kreis von Leuten. Zwar blieben die Wissenschaftlerinnen und Wissenschaftler an wissenschaftlichen Symposien unter sich, zunehmend fand jener von der FAL angestrebte Austausch aber statt. Mit dafür verantwortlich war die Koinzidenz von Curriculumforschung und politisch forcierter Bildungsreform durch Lehrplanrevisionen in den 1970er-Jahren. Sie ermöglichte erst bestimmte Formate des Wissensaustausches: Konferenzen, Arbeitsgruppen, Projektteams. Bildungsreformen gewannen dadurch an wissenschaftlicher Unterfütterung und Legitimation, die Ansätze der Curriculumforschung als Teil der aufstrebenden Bildungsforschung im Gegenzug Anwendungszusammenhänge. Anders als die vornehmlich geisteswissenschaftlich ausgerichteten Professuren reagierte die Freiburger Arbeitsgruppe mit ihren sozialwissenschaftlich orientierten Modellen geschickt auf den bildungspolitischen Bedarf an Problembearbeitung. Curriculumforschung avancierte zum politischen Instrument.

I74 Strittmatter, Vorfragen an potentielle Auftraggeber für Projekte, Gutachten, Beratungen, Kurse, 28. 3. 1973, Archivalien der FAL.

175 Heinze, 2012. 


$$
\text { (name }
$$




\section{Lehrplanreformprojekte: Realisierung von Forschungskonzepten}

Auf die Dauer werden daher die Universitäten nicht all die Planungs- und Verwaltungsarbeit übernehmen wollen, die mit einem grösseren Curriculumprojekt unvermeidlich verbunden ist. ${ }^{\mathrm{T}}$

Während in bildungspolitischen Debatten der I970er-Jahre um konsensuale Positionen in Koordinationsfragen gerungen wurde und sich zunehmend die Lehrpläne als Gegenstand der Reformintentionen entpuppten, leiteten die Kantone dem gemässe, gross angelegte Bildungsreformprojekte ein, die teilweise bis in die frühen I990er-Jahre andauerten. Mit der Projektdurchführung wurden oftmals eigene Planungs- und Verwaltungsstellen betraut, welche ihrerseits die Lehrpersonen in die Lehrplanarbeiten involvierten.

Das vorliegende Kapitel untersucht vier ausgewählte Bildungsreformprojekte und interessiert sich übergeordnet für die Rezeption der in den Debatten verhandelten Wissensbestände mit curriculumtheoretischer Provenienz. Diese Prozesse sind vor dem Hintergrund von drei bereits im Vorkapitel ausgebreiteten Kontexten zu verstehen. Erstens unterlag das öffentliche, kantonal organisierte Schulwesen in den I970er-Jahren einer starken Koordinationstendenz, wobei Lehrpläne zu einem zentralen Element der bildungspolitischen Bestrebungen wurden. Darüber hinaus sind zweitens die bildungspolitischen Entwürfe der Projektarbeiten und deren Umsetzungen geprägt von Fortschrittsorientierung und Planungsgläubigkeit. Nicht zuletzt diesem Umstand ist das Bedürfnis nach wissenschaftsbasierter Beratung und Expertise geschuldet. ${ }^{2}$

Mit Blick auf den Kanton Basel-Stadt, die Zentralschweiz, den Kanton Freiburg und ein interkantonal angelegtes Projekt der EDK werden aus der Perspektive der je beteiligten Kantone die Auslöser für die jeweiligen Reformprojekte eruiert, die federführenden Akteure ermittelt und die Argumente ihrer Konzepte beleuchtet. Es gilt sodann die gewählten Formen der Projektumsetzung zu situieren und auf ihre Bedeutung und ihre Konsequenzen für die Lehrplanarbeit, die Lehrpläne und deren Funktion zu befragen. Letztlich wird eruiert, inwiefern Curriculumforschung nicht bloss geschriebenes und diskutiertes

I Huhse, I968, S. 53.

2 Weiterführend Kapitel 3.I. 
Wort blieb, sondern in bildungspolitischen Reformprojekten handlungsleitend wurde und auf die Forschung zurückwirkte.

\section{1 «Erziehungswissenschaftliche Kapazitäten» in Lehrplanreformprojekten des Kantons Basel-Stadt}

Für nicht ausgeschlossen halten wir es, dass in Zukunft jene, die durch Sprayaktionen öffentliche Bauten verunstalten, sich darauf berufen, das Bemalen von Wänden gehöre zum Lehrplan der staatlichen Realschule. ${ }^{3}$

Das erste Teilkapitel analysiert Lehrplanreformprojekte im Kanton Basel-Stadt von 1968 bis in die frühen I980er-Jahre. In die Projekte waren der kantonale Erziehungsrat, ${ }^{4}$ die Schulleitenden auf der Sekundarstufe I, die Lehrpersonen, die Bildungsverwaltung und wissenschaftliche Experten involviert, nicht zuletzt auch die breite Öffentlichkeit aufgrund der politischen Dimension, welche der Bestimmung schulischer Inhalte inhärent ist, wie das einleitende Zitat verdeutlicht.

Im Zentrum der Analysen steht die Frage, inwiefern in den langwierigen Lehrplanreformprojekten des Kantons Basel-Stadt auf Erkenntnisse aus der Curriculumforschung zurückgegriffen wurde. Wer waren die federführenden Akteure, auf welche Wissensbestände haben sie sich in Empfehlungen oder Konzeptarbeiten bezogen, und inwiefern sind Letztere realisiert worden?

Die ersten beiden Kapitel analysieren die Lehrplanreformprojekte im Kanton Basel-Stadt exemplarisch an den Arbeiten zum Lehrplan der Basler Realschulen bis in die frühen I980er-Jahre. Sie arbeiten jenen bedeutenden Bruch in der Lehrplanarbeit um 1975 heraus, welcher in den darauffolgenden Teilkapiteln das Institut für Unterrichtsfragen und Lehrerfortbildung (ULEF) und Karl Frey als Experten im Bereich der Curriculumkonstruktion in den Fokus rückt.

3 Hans Löffler, Betreffend Lehrplanreform Realschule BS, 28. 2. 1982, EDBS, Reg. Nr. 430/r. Hervorhebung im Original.

4 Der Erziehungsrat (auch Bildungsrat, Erziehungskommission, Landesschulkommission, Studienkommission) ist ein in den meisten Kantonen bestehendes Laiengremium, welchem in Abgrenzung von der Kantonsregierung die Aufsicht über das kantonale Bildungswesen zukommt. Je nach Kanton ist seine Funktion unterschiedlich weit ausgelegt und es sind ihm Beratungs-, Regelungs- bis hin zu Erlasskompetenzen übertragen, insbesondere hinsichtlich Lehrmitteln und Lehrplänen. Weiterführend Plotke, I979 S. 324326. 


\subsubsection{Lehrplanreformen zur Umsetzung der Koedukation}

Am I4. März I968 beschloss der Grosse Rat's des Kantons Basel-Stadt die Einführung der Koedukation für die Realschulen und die Sekundarschulen. ${ }^{6}$ Die Umsetzung wurde auf das Schuljahr I970/71 festgelegt, weshalb im Juni I968 eine zwölfköpfige Lehrplankommission unter der Leitung des damaligen Rektors der Knabenrealschule Hermann Jud die Ausarbeitung eines neuen Lehrplans aufnahm. ${ }^{7}$ Während neun Sitzungen hatte sich die Kommission über ihrer Aufgabe aber derart zerstritten, dass die Basler «National-Zeitung» und die «Basler Nachrichten» im Frühjahr 1969 berichteten: «Es stinkt im Erziehungsrat», «Es harzt bei der Realschul-Koedukation» und «Was alles in unserem Staate geschehen kann». ${ }^{8}$ Ein Teil der Kommissionsmitglieder hatte offenbar die Bemühungen einer eindeutigen Umsetzung der Koedukation in den Basler Realschulen unterlaufen wollen. Der Regierungsrat empfing in der Folge zwei Zwischenberichte, einen von der Kommission und einen der Kommissionsminderheit. Er löste aufgrund der Ungereimtheiten die Kommission per Beschluss vom i 8 . Februar I969 auf, um sie durch eine bedeutend kleinere mit neuen Delegierten zu ersetzen.

Das Ziel bestand nach wie vor darin, per Schuljahr I970/7I mit dem ersten Jahrgang an einer koedukativen Realschule zu starten. Der neue Kommissionspräsident liess verlauten, «dass die nun zu treffende Lösung des Koedukationsproblems an den Realschulen eindeutig den Charakter eines Provisoriums trägt».9 Er ergänzte zudem mit Blick auf das medienwirksame Scheitern der vorhergehenden Lehrplankommission: «Die mögliche Verwirklichung neuer Erkenntnisse in den Lehrplänen anderer Schulen in und ausserhalb unseres Kantons darf uns nicht davon abhalten, heute für unsere Realschulen eine Lösung vorzuschlagen, die mit einem Minimum an Unruhe und Unsicherheit morgen gangbar ist.» ${ }^{10}$

5 Der Grosse Rat ist das Parlament des Kantons Basel-Stadt.

6 Auftakt zur gemeinsamen Beschulung von Mädchen und Knaben für alle Schultypen der Sekundarschule I war der Ratschlag 63 I betreffend die Einführung der Koedukation vom I0. I I. I966. Die folgenden Ausführungen konzentrieren sich auf die Reformen der Realschulen Basel-Stadt. Für die Sekundarschulen des Kantons verlief parallel dazu ein vergleichbarer Prozess.

7 P. Böhringer, Kommission zur Vorbereitung der Lehrpläne der koeduzierten Realschule, I4. 3. 1972, StABS, ED REG 5a.

8 National-Zeitung, I 3. 4. 1969; Basler Nachrichten, 24. 4. I969; Basler Nachrichten, 4. 4. I969, StABS, ED REG sa.

9 P. Böhringer, Neue Kommission zur Vorbereitung der Lehrpläne der koeduzierten Realschule, 2. I0. 1969, StABS, ED REG sa.

Io Ebd. 
Auf Experimente wollte man sich nicht einlassen, ein grosser Wurf sollte der neue Lehrplan der koedukativen Realschulen demnach gar nicht werden. Das ausgearbeitete Endresultat wurde in der Vernehmlassung ${ }^{\text {II }}$ entsprechend kritisch diskutiert, etwa von Heinz Rupp von der Universität Basel. Der Professor für Germanistik bemerkte zum Lehrplan im Fachbereich Deutsch: «Der Lehrplan-Entwurf, hält man sich strikt an die Formulierung, ist, vom heutigen Stand der Wissenschaft aus gesehen und sanft formuliert, veraltet.» ${ }^{\mathrm{I} 2}$ Dennoch genehmigte der Erziehungsrat im März I 972 den ausgearbeiteten Lehrplan und setzte ihn für die folgenden vier Jahre provisorisch in Kraft.

Diverse Fachkommissionen und die Konferenzen der Lehrpersonen unterzogen den Lehrplan im Hinblick auf das Auslaufen des Provisoriums im Sommer 1975 einer kritischen Prüfung. Sie kamen zum Schluss: «Die Lehrziele und Stoffpläne der Realschule sind noch brauchbar, aber in mancher Hinsicht nicht mehr zeitgemäss.»'33 Gestützt auf die Schulinspektion und die Lehrpersonen stellte Hermann Jud als Rektor der Realschule Basel-Stadt an den Erziehungsrat den Antrag auf Verlängerung des Provisoriums mit der Begründung: «Wissenschaftliche Erkenntnisse der letzten Jahre zwingen uns zum weitgehenden Umdenken bei der Lehrplangestaltung. In zentralen Fächern wie Mathematik, Deutsch und Fremdsprachen werden völlig neue Wege eingeschlagen, die über kurz oder lang in den Stoffplänen ihren Niederschlag finden müssen. Die Notwendigkeit einer Neukonzeption der Lehr- resp. Lernziele und der Stoffpläne der Realschule liegen somit auf der Hand.» ${ }^{14}$

In demselben Schreiben nahm der Rektor zudem Bezug auf den beiliegenden Antrag der Konferenz der Realschullehrpersonen, wonach bald eine i 5 -gliedrige Kommission mit der «Totalrevision der Lehrziele und Stoffpläne» zu beauftragen sei. ${ }^{\text {is }}$ Der Erziehungsrat folgte den Antragstellenden und leitete per Beschluss vom I. Oktober I975 eine weitere Phase der Lehrplanarbeit im Kanton Basel-Stadt ein.

Zusammenfassend betrachtet waren die Lehrplandebatten bis 1975 gekennzeichnet von Gestaltungsfragen bei den Stoffplänen und den Stundentafeln. Die Zusammenführung des Unterrichts von Schülerinnen und Schülern stellte die Kommissionen vor die Aufgabe, die teilweise unterschiedlichen Lehrpläne und Fächerdotationen abzugleichen. Beispielsweise wurde Geometrie in der dritten

Die Vernehmlassung ist eine Phase im schweizerischen Gesetzgebungsverfahren. Dabei wird Verbänden, Parteien und anderen interessierten Kreisen eine Vorlage ausgehändigt, verbunden mit der Aufforderung zur Stellungnahme über sachliche Richtigkeit, Vollzugstauglichkeit und Akzeptanz. Weiterführend www.admin.ch.

Hein Rupp, 4. 2. 1972, StABS, ED REG sa.

Hermann Jud, 22. 8. 1975, StABS, ED REG sa.

Ebd.

Ebd. 
und vierten Klasse der Realschule für Mädchen bis dahin gar nicht erteilt. Im Vordergrund stand das Ringen um Kompromisse etwa zur Frage, wie viele Wochenstunden dem Fachbereich Handarbeit, Werken und Gestaltung zugeteilt werden müssen und wie sie auf die Mädchen und die Knaben aufzuteilen seien. In diesem Fachbereich wie auch im Sport wurde die Koedukation vorerst nicht vollständig umgesetzt. ${ }^{16}$ Die Diskussionen über die Stoffpläne wurden vergleichsweise beiläufig geführt. Sie waren geprägt von der Festlegung geeigneter Lehrmittel und der Aufteilung von Unterrichtsthemen auf die einzelnen Schuljahre. Im Fach Geschichte orientierte man sich an den Empfehlungen einer interkantonalen Kommission zur Vereinheitlichung der Lehrpläne und Lehrziele und hielt letztlich im Lehrplan fest: «Entwicklungsgeschichtlicher Überblick (Wohnung, Ernährung, Kleidung, Schrift etc.), Beispiel eines alten Kulturvolkes, Bedeutung der Antike für das Abendland, Das Christentum, Die Römer am Oberrhein und die Kelten (Helvetier) [...]» ${ }^{17}$ Diskussionen über Lehrziele einzelner Fachbereiche liegen in den umfassenden Dokumenten der Vernehmlassung kaum vor. Die Lehrziele waren in den Lehrplänen generell sehr allgemein gehalten, wie das folgende Beispiel, wiederum aus dem Fachbereich Geschichte, zeigt: «Ziel: Verständnis der heutigen politischen kulturellen, sozialen und wirtschaftlichen Verhältnisse aus der geschichtlichen Entwicklung.» ${ }^{18}$

\subsubsection{Neue Prinzipien der Lehrplanarbeit ab 1975}

Die Lehrpläne der Realschule im Kanton Basel-Stadt beinhalteten bis 1975 als gestaltende Elemente knappe Lehrziele, stichwortartige Stoffpläne und eine Stundentafel zur Bestimmung der Unterrichtsdauer der einzelnen Fachbereiche. Bis dahin waren Lehrpläne Dokumente, welche sich in ihrer Aufmachung nur geringfügig veränderten. Doch nach weiteren sechs Jahren der Lehrplanarbeit im Kanton Basel-Stadt kündigte der Vorsteher des Erziehungsdepartementes Arnold Schneider am I8. August I98 I in aller Deutlichkeit einen Wandel an: Er empfahl den Mitgliedern des Erziehungsrates die Genehmigung des neuen Lehrplanes für die Realschulen, wobei er hervorhob: «Der neue Lehrplan unterscheidet sich in verschiedenen Punkten sehr stark vom bisherigen. ${ }^{19}$ Die Veränderungen im neuen Lehrplan seien in drei Bereichen sichtbar: dem

I6 Schlussbericht der Kommission zur Vorbereitung der Lehrpläne der koeduzierten Realschule, I 4. 3. 1972, StABS, ED REG sa.

I7 Lehrziele und Stoffpläne für die koeduzierte Realschule Basel, I. Teil: I. und 2. Klassen, 5.3. 1970, StABS, ED REG sa.

I8 Ebd.

I9 Arnold Schneider, Neuer Lehrplan für die Realschule, i 8. 8. I98 I, StABS, ED REG sa. 
Umfang, der Inhaltsaufbereitung sowie der Kommissionszusammensetzung. Sie werden nachfolgend kurz ausgeführt.

Auf den ersten Blick fiel die Veränderung des Umfangs auf: «Der bisherige Lehrplan umfasste 44 lose bedruckte Seiten. Der neue muss in Form eines Ordners abgegeben werden. $»^{20} \mathrm{Er}$ wurde angereichert mit Überlegungen zur Stoffauswahl und Zielformulierungen, nicht zuletzt aufgrund eines neuen Vorgehens bei der Lehrplankonstruktion: «Der bisherige Lehrplan bestand aus stichwortartigen Zielen [...] und aus sehr summarischen Stoffangaben [...]. Demgegenüber geht der neue Lehrplan von Leitideen aus, die den Sinnzusammenhang des Unterrichts mit dem Leben in unserer Zeit und in unserer Gesellschaft hervorzuheben versuchen. Der Lehrplan enthält auch viele praktische Hinweise dazu, wie diese Leitideen in Unterricht umgesetzt werden können. In einer letzten Phase der Konkretisierung findet man dann wieder Stoffprogramme.» ${ }^{21}$

Leidideen wurden einerseits als «didaktische Leidideen» in der Einleitung des neuen Lehrplans platziert und lauteten beispielsweise: «Die Schule soll in gewissem Umfang ein eigenbestimmter Lebensraum für Schüler und Lehrer sein. $\gg^{22}$ Diesem Umstand sei in der Gestaltung des Unterrichts Rechnung zu tragen. Andererseits führten Leitideen die Lesenden in die fächerspezifischen Abschnitte des Lehrplans ein, bevor daraus abgeleitete Ziele und Aufgaben des Unterrichts erläutert wurden. In der Mathematik lautete eine Leitidee etwa: «Die Lernsituationen müssen für den Schüler aus dem Alltag stammen; sie sollen ihn packen und zum Mitmachen bewegen. Der Unterricht wird zum Anreiz für Tätigkeiten.» ${ }^{23}$

Der neue Lehrplan für die Realschulen beinhaltete zusätzlich das allen Fächern übergeordnete Erziehungsziel, «die Schülerinnen und Schüler zu mündigen Menschen zu erziehen». ${ }^{24}$ Mündigkeit wurde weiter definiert durch die Fähigkeit zur Selbstbestimmung, durch die Fähigkeit zu verantwortungsbewusstem Handeln und durch das Verfügen über Denkvermögen und Fachwissen zur Handlungs- und Entscheidungsfähigkeit, was je mit Selbstkompetenz, Sozialkompetenz und Sachkompetenz präzisiert wurde. Mit diesem einleitenden Erziehungsziel orientierte sich die Lehrplankommission überdies wortwörtlich an den publizierten Diskussionsergebnissen einer Tagung in Freiburg zu Leitideen anlässlich des SPIPRI-Projektes; dazu später in diesem Kapitel. ${ }^{25}$

Ebd.

Ebd.

Lehrplan Realschule Basel-Stadt. Revidierte Fassung von 1985, EDBS, S. 2, Reg. Nr. $430 /$ I.

Ebd., S. 52.

Ebd., S. I.

A. Oggenfuss, 1983, S. 73 . 
Die dritte Neuerung betraf den Prozess der Erarbeitung des neuen Lehrplans für die Realschulen. Nachdem die Lehrplankommission von 1969, bedingt durch innere Zerwürfnisse, aufgelöst worden war, erarbeitete die kleinere Folgekommission das bis I98 I gültige Provisorium weitgehend ohne Mitwirkung von Lehrpersonen. Der von 1975 bis I98 I ausgearbeitete Lehrplan «kam unter wesentlich breiterer Beteiligung der Lehrerschaft zustande. Er spiegelt die heutigen Vorstellungen der Lehrerschaft über einen modernen und sinnerfüllten Unterricht wider», ${ }^{26}$ wie der Vorsteher des Erziehungsdepartements Arnold Schneider betonte.

In der Vernehmlassung zum Lehrplan von 198 I nicht unumstritten blieb die Neuerung, Lernziele für die einzelnen Fachbereiche zu formulieren. Lernziele sind nicht zu verwechseln mit den oben angeführten Lehrzielen, welche bereits in älteren Lehrplänen vorlagen und sich durch tendenziell vage Beschreibungen auszeichneten. Lernziele waren bedeutend konkreter formuliert. Für den Umgang mit Texten im Deutschunterricht wurden beispielsweise folgende Lernziele vorgegeben: «Der Schüler soll Lesetexte vorausschauend, flüssig, lautrein und sinnerschliessend vortragen können; der Schüler soll lernen, den Inhalt zu erfassen, wiederzugeben und die Bedeutung von Einzelstellen für das Ganze zu erklären und zu beurteilen.» ${ }^{27}$

Lernziele waren im vorliegenden Fall auf bestimmte Fähigkeiten und Fertigkeiten hin ausgerichtet, welche die Schülerinnen und Schüler erwerben sollten. Den Lehrpersonen eröffneten sie Handlungsspielräume in der Bestimmung von Unterrichtsinhalten überall dort, wo der Lehrplan keine weiteren stofflichen Vorgaben direkt an die jeweiligen Lernziele knüpfte. Darauf zielte etwa die folgende Kritik anlässlich der Vernehmlassung im Februar 1982 ab: «Bei der vorliegenden Form, in welcher der Lehrer in jeder Klasse nach freiem Ermessen den Stoff auswählen kann, ist jeder Klassenwechsel (Zuzug nach Basel, Wegzug von Basel, Schulwechsel, Remotion, Klassenauflösung, Wechsel des Wohnquartiers etc.) für den Schüler eine Katastrophe.» ${ }^{28}$

Zusammenfassend zeigt dieser kurze Abriss über die von I968 bis I98 I dauernde Ausarbeitung neuer Realschullehrpläne im Kanton Basel-Stadt einen deutlichen Bruch in der Historie der Basler Lehrplanarbeit. Ausgehend vom schulstrukturell und gesellschaftspolitisch bedingten Bestreben, die Schülerinnen und Schüler koedukativ zu unterrichten, mussten die Lehrpläne und an erster Stelle die Stundentafeln revidiert werden. Im Zuge der Reformprozesse wurde von verschiedenen Seiten bemerkt, dass über die Stundentafeln hinaus

26 Arnold Schneider, Neuer Lehrplan für die Realschule, I8. 8. I98 I, StABS, ED REG sa.

27 Lehrplan Realschule Basel-Stadt. Revidierte Fassung von 1985, S. I r, EDBS, Reg. Nr. $430 /$ I.

28 Hans Schaub, Zum neuen Lehrplan der Realschule, I3. 2. I98 I, EDBS, Reg. Nr. 430/ I. 
ebenso Reformbedarf bei Lehrzielen und Stoffplänen bestehe und dass dies mitunter wissenschaftlich angezeigt sei. Reformdruck aufgrund auslaufender Fristen von provisorisch eingesetzten Lehrplänen, gekoppelt mit politischer Zurückhaltung bei gewagten Grossprojekten - eine weitere Negativkampagne in den Medien galt es zu vermeiden -, erlaubte erst ab i975 eine Aufnahme von umfassenden und grundlegenden Reformen. Dann schien es angezeigt, bestehende Stoffpläne grundsätzlich zu hinterfragen, neue Prinzipien auszuarbeiten und mit grösserer Beteiligung der Lehrpersonen in der zuständigen Kommission zu tagen. Elemente wie Leitideen, Lernziele und übergeordnete Erziehungsziele wurden ausgearbeitet und davon ausgehend die Auswahl von Unterrichtsinhalten vorgenommen.

Die Arbeiten zum Lehrplan der Realschulen waren mit der im November I98 I vom Erziehungsrat bewilligten Fassung noch nicht abgeschlossen. Bis in die späten I980er-Jahre wurden Teilbereiche des Lehrplans weiterbearbeitet. Insbesondere die einleitenden Grundsätze zur Realschule im Kanton Basel-Stadt wurden bis in die späten I980er-Jahre zum Gegenstand längerer Debatten zwischen dem Erziehungsrat und den involvierten Lehrpersonen. Darüber hinaus forderte der Erziehungsrat eine Ergänzung des Lehrplans durch einen für die Lehrpersonen verbindlichen, übersichtlichen Stoffplan. Die neuen Kernelemente wie Leitideen und Lernziele blieben aber weitgehend unumstritten. ${ }^{29}$ Die folgenden Ausführungen nehmen die grundlegende Veränderung der Lehrplankonstruktion Mitte der I970er-Jahre zum Ausgangspunkt und fragen nach den Mechanismen und Bedingungen, welche diesen Wandel ermöglicht haben. Es steht insbesondere die Frage im Fokus, wie die Informationen, die Ideen und damit das Wissen über neue Konzepte der Lehrplankonstruktion an die Lehrplankommission und an die weiteren Involvierten gelangt sind.

\subsubsection{Das Institut für Unterrichtsfragen und Lehrerfortbildung (ULEF)}

Im März 1968 genehmigte der Erziehungsrat des Kantons Basel-Stadt eine neue Ordnung für das Institut für Unterrichtsfragen und Lehrerfortbildung (ULEF). ${ }^{3 \circ}$ Im ersten Paragrafen wird der Auftrag des Instituts festgelegt als «Aufgabe, den Behörden, der Lehrerschaft und den Eltern vom Stand und von den Bestrebungen der Schule Kenntnis zu geben und die Weiterbildungen der Lehrer zu fördern, indem es Gelegenheit bietet, Neuerungen im Schulwesen

29 Arnold Schneider, Lehrplanreform an der Realschule, 29. 9. I982, EDBS, Reg. Nr. 430/ I.

30 Das ULEF hat sich ausgehend von der Basler Schulausstellung von 1924 stetig weiterentwickelt, bis 1968 eine Anpassung ihrer Ordnung vorgenommen wurde. Weiterführend Rüegg, Dunkel und Schuppli, 2009. 
vorzuführen, zu studieren und zu fördern».31 Das ULEF veranstaltete hauptsächlich Kurse, Vorträge, Lehrproben und Exkursionen für Lehrpersonen und weitere Interessierte. Darüber hinaus war es verschiedentlich in die schulpolitischen Entwicklungen im Kanton Basel-Stadt involviert, so auch in die Lehrplanreformen der Real- und Sekundarschulen.

Der Direktor des ULEF, Guido Harder, ${ }^{32}$ notierte im Jahresbericht von I97 I eine Übersicht über die im Berichtsjahr durchgeführten Vorträge. Fünf Referate thematisierten die Vorschulerziehung und verschiedene Kindergartenmodelle, es wurde «zum Selbstverständnis des Lehrers» oder «die Ausbildung des Primarlehrers» gesprochen, aber auch «Ergebnisse der denkpsychologischen Forschung und ihre Bedeutung für den Unterricht» wurden präsentiert und weitere fachwissenschaftliche Vorträge gehalten. ${ }^{33}$ Sie alle waren mit bis $\mathrm{zu} 300$ Zuhörenden gut besucht und wurden von anerkannten Referierenden vorgetragen. ${ }^{34}$

Daneben lancierte das ULEF sogenannte Werkstattseminare. Die an solchen Seminaren Teilnehmenden - in der Regel Lehrpersonen oder Ausbildnerinnen und Ausbildner an Lehrerinnen- und Lehrerseminaren - erhielten die Gelegenheit, in frei wählbaren Projektgruppen während eines oder mehrerer Tage sich neues Wissen anzueignen. Im Spätsommer 1972 veranstaltete das ULEF beispielsweise ein Werkstattseminar mit den Projektgruppen «Spracherziehung, Mathematik, Kunst- und Werkerziehung, Sachkundliche Erziehung, Allgemeine Didaktik» sowie einer «Projektgruppe für spezielle Fragen».35 Anlässlich eines Referates vor der Aufsichtskommission des ULEF lobte Karl Ingold als Mitorganisator dieser Weiterbildungsveranstaltung die Arbeit in Projektgruppen und betonte: «Nur durch eine solche Aktivierung der Teilnehmer ist es möglich, den verschiedenartigen Eindrücken und Erkenntnissen zum wirklich bleibenden Durchbruch zu verhelfen. Dabei ist es allerdings von absoluter Notwendigkeit, dass [...] Tutoren zur Verfügung stehen, welche die persönliche und fachliche Kompetenz in hervorragender Weise gewährleisten.»36

3 I Ordnung für das Institut für Unterrichtsfragen und Lehrerfortbildung des Kantons Basel-Stadt vom I4. 2. 1968, Archivalien des ULEF.

32 Guido Harder wurde 1969 vom Erziehungsrat des Kantons Basel-Stadt zum ersten vollamtlichen Direktor des ULEF gewählt. Er hatte dieses Amt bis zu seinem frühen Tod I980 inne.

33 Jahresbericht des Vorstehers, I97I, Institut für Unterrichtsfragen und Lehrerfortbildung, S. I8, Archivalien des ULEF.

34 Unter den Referierenden im Jahr I97 I waren etwa Rudolf Bergius, Universität Tübingen, Reinhard Tausch, Universität Hamburg, oder Rudolf Schenkel, Universität Basel.

35 Karl Ingold, Zusammenfassende Orientierung über das Werkstattseminar für Lehrkräfte vom 21. 8. bis I. 9. 1972, Archivalien des ULEF.

36 Ebd. 
Ingold insistierte auf der Bedeutung der beigezogenen Tutoren für die Werkstattseminare. Im Falle der Veranstaltung von 1972 waren das Rudolf Messner, Horst Rumpf und Klaus Heipcke als etablierte Didaktiker für die Gruppe «Spracherziehung», Heinrich Bauersfeld vom Institut für Mathematikdidaktik in Bielefeld für die Gruppe «Mathematik», Gunter Otto als Kunstpädagoge und Didaktiker für die Gruppe «Werkerziehung», die Professoren für Erziehungswissenschaft in Hamburg Karl-Heinz Flechsig und Wolfgang Schulz für die Gruppe «Allgemeine Didaktik» sowie Karl Frey vom Leibniz-Institut für die Pädagogik der Naturwissenschaften (IPN) für gleich drei Gruppen: «Allgemeine Didaktik», «Projektgruppe für spezielle Fragen» und «Sachkundliche Erziehung». Insgesamt engagierte das ULEF für diese und einige weitere Weiterbildungsveranstaltungen je eine illustre Gruppe von Experten für Unterricht und Didaktik.

Der Umstand, dass das ULEF in Basel und insbesondere dessen Direktor Guido Harder angesehene Autoritäten ausgewählter Fachbereiche für Seminare und Referate zu gewinnen wusste, hatte sich offenbar auch in Kreisen der Lehrerinnen- und Lehrerbildung ausserhalb beider Basel herumgesprochen. So ist im Jahresbericht von 1979 über ein Treffen in Basel zu lesen, welchem die Leiterinnen und Leiter aller schweizerischen Institutionen beiwohnten, an denen Lehrpersonen für die Sekundarstufen I und II ausgebildet wurden. Zu diesem Treffen vermerkte der Präsident der Aufsichtskommission des ULEF im Jahresbericht, Guido Harder sei attestiert worden, die erziehungswissenschaftliche Weiterbildung der Dozierenden vorzüglich voranzubringen. Er ergänzte: «Da mit wenigen Ausnahmen die Universitäten der Schweiz und insbesondere Basel bis vor kurzer Zeit schlecht für die erziehungswissenschaftliche Ausformung von Ausbildnern eingerichtet waren, stellte der Beizug namhafter Professoren aus dem In- und Ausland am ULEF eine Art «Pilot-Universität für Ausbildner dar.»37

Während des Besuchs der Ausbildungsleitenden im Basler ULEF befand sich die Reform der Lehrpläne für die Realschulen des Kantons Basel-Stadt in jener Phase, welche sich durch einen Einbezug von neuen Elementen und Gestaltungsideen auszeichnete, aber auch durch eine weitgehend von Lehrpersonen gestemmte Lehrplanarbeit. Im Jahresbericht von 1979 steht dazu: «Die Anpassung der «Didaktischen Analyse an unsere Bedürfnisse, die Entwicklung offener Curricula an der Stelle von starren, zwanghaften, den Lehrer entmündigenden Unterrichtszwängen sind Beispiele für fruchtbares Weiterdenken in den ULEF-Veranstaltungen.» ${ }^{8}$ 
Dieses Zitat verdeutlicht, dass das ULEF die Zügel der Lehrplanreformen mindestens im operativen Bereich in den Händen gehalten hat. Es wird den ULEF-Veranstaltungen die Qualität zugesprochen, neue Curriculumkonzepte in die laufenden Reformen einzubringen und sie gemäss den Ansprüchen der Basler Schulen zu adaptieren. Mit dem Tod des Direktors Guido Harder 1980 musste die Rolle des ULEF aber neu ausgehandelt werden. Als der Erziehungsrat des Kantons Basel-Stadt im Oktober 1982 auch für die Sekundarschulen einen neuen, verbindlichen Lehrplan wünschte, waren wohl die Inspektoren der Sekundarschulen, die Schulrektorate und die Projektleitung informiert, das ULEF wurde aber nicht darauf angesprochen. Im Protokoll der Arbeitstagung des ULEF vom Juni 1983 ist demgemäss vermerkt: «Die Stellung des ULEF im Rahmen der Lehrplanreform ist unklar definiert. Es liegt weder ein Auftrag der vorgesetzten Gremien vor, noch ist das ULEF Gesprächspartner dieser Gremien. Andere Kantone (z. B. Bern, Freiburg, Zürich, Baselland, Solothurn) kennen eigentliche «Leiter von Lehrplanprojekten〉 oder «Wissenschaftliche Mitarbeiter, die nichts anderes tun, als zu einschlägigen Themen Begleituntersuchungen durchzuführen.»39

Die ULEF-Kommission fasste an ihrer Arbeitstagung nach eingehender Diskussion dieses Befundes den Beschluss, wieder eine aktive Rolle in den Lehrplanreformen übernehmen zu wollen, und auferlegte sich die folgenden durch den Erziehungsrat noch zu genehmigenden neuen Aufträge: erstens die Einführung der neuen Lehrpläne zu unterstützen, zweitens die Reformkommissionen in der Leitideendiskussion durch Öffentlichkeitsarbeit zu unterstützen, drittens die Information der Bildungsausschüsse der politischen Parteien zu unterstützen. ${ }^{\circ}$ Harder hatte einst im ersten Paragrafen der Ordnung des ULEF erfolgreich den Zusatz einfügen können, dass auch «wissenschaftliche Untersuchungen» im Auftrag des ULEF zu erbringen sind. Aus personellen und finanziellen Gründen war man aber der Ansicht, dies derzeit nicht leisten zu können. ${ }^{41}$

Insgesamt betrachtet ist es weitgehend Guido Harders Verdienst, dass das ULEF in den Lehrplanreformen bis 1980 sichtbar und aktiv positioniert war. Er lancierte Arbeitsgruppen, stand mit den Schulrektoraten und dem Erziehungsrat im Austausch und verfügte über gute Kontakte in die Wissenschaft, mit deren Hilfe er neue Impulse in die laufenden Reformprojekte einzuflechten vermochte. Ein Beschwerdebrief des Präsidenten der ULEF-Kommissi-

39 Protokoll der Arbeitstagung der ULEF-Kommission, 23.-25.6. 1983, S. 5, Archivalien des ULEF.

40 Ebd.

4I Protokoll der Arbeitstagung der ULEF-Kommission, 23.-25.6. 1983, Archivalien des ULEF. 
on Hanspeter Müller ${ }^{22}$ an Harder zeugt von dessen teilweise sehr geschickter Vorgehensweise zur Organisation von Tagungen und Weiterbildungen, um die Themensetzung in seinem Sinne vorzunehmen. Jedenfalls konnte Harder so die eigene thematische Agenda realisieren und das ULEF während seiner Amtszeit als Schnittstelle und Vermittlungsinstanz zwischen erziehungswissenschaftlicher Forschung, bildungspolitischen Reformprojekten und der Aus- und Weiterbildung von Lehrpersonen positionieren.

Seine umtriebigen Tätigkeiten wurden ferner bis über die Landesgrenzen hinaus wahrgenommen: «Auch die Lehrplan-Entwicklung als Gross-Projekte ganzer Konferenzen, ebenfalls unter der Leitung erziehungswissenschaftlicher Kapazitäten, hat unter den Fachleuten im Ausland hohe Beachtung gewonnen.»33 Insbesondere Karl Frey, Gründer der FAL und ab I97I Leiter des IPN in Kiel, ist zu den oben genannten «erziehungswissenschaftliche[n] Kapazitäten» zu zählen. Er wurde vom ULEF mehrfach in die Lehrplanreformprojekte mit einbezogen und wirkte massgeblich an den Reformen ab 1975 mit, wie nachfolgend dargelegt wird.

\subsubsection{Karl Frey in den Reformprojekten des Kantons Basel-Stadt}

Im Juni 1975 verfassten Guido Harder und zwei Mitarbeitende gemeinsam mit Karl Frey das Konzept für die Lehrplanarbeit des neu zu erstellenden Realschullehrplanes, welches weitgehend als Drehbuch für die Folgejahre fungieren sollte. Unter dem Titel «Projektbeschreibung für die Lehrplanüberarbeitung der Realschulen im Kanton Basel-Stadt» ${ }^{44}$ wurden sieben Arbeitsprinzipien festgelegt, die Lehrplanarbeit in verschiedene Phasen aufgegliedert sowie die Arbeit in Teilgebiete - womit explizit nicht Unterrichtsfächer gemeint waren und erste Vorschläge zur Erarbeitung von Leitgedanken und Unterrichtsprinzipien der Realschule vorgelegt. Im Folgenden werden zentrale Elemente des Konzepts kurz umrissen.

Das erste und wichtigste Arbeitsprinzip wies den allgemeinen Leitgedanken und Unterrichtsprinzipien der Realschule eine zentrale Funktion zu. Sie sollten als Erstes ausgearbeitet werden. Sämtliche weiteren Arbeiten in Fachgruppen würden sich dann an den Leitgedanken orientieren. Eine ständige «Grundsatz-

42 Hanspeter Müller war zunächst Lehrer am Mädchengymnasium in Basel. 1956 wählte ihn der Regierungsrat zum Direktor des kantonalen Lehrerseminars, welches er bis Oktober I 980 leitete. Er war zudem Direktor der ULEF-Kommission.

43 H. P. Müller, Jahresbericht 1979, 6. 8. 1979, Archivalien des ULEF.

44 Lehrplanüberarbeitung der Realschule im Kanton Basel-Stadt, Projektbeschreibung, I0. 5. 1976, Archivalien des ULEF. 
gruppe» war zur Koordination mit den weiteren Arbeitsgruppen vorgesehen. Anders als in bisherigen Lehrplanreformen standen nicht die Stundentafeln oder die Stoffpläne am Anfang der Lehrplanarbeit.

Die Arbeit wurde als Gruppenarbeit mit fünf bis zehn Lehrpersonen konzipiert und ihr wurde ein Jahresrhythmus unterlegt, wobei die Zwischenergebnisse aus den Gruppen «evaluiert» wurden und halbjährlich im Plenum aller Arbeitsgruppen zur Diskussion vorzulegen waren. Zudem war vorgesehen, dass in jeder Arbeitsgruppe «mindestens $30 \%$ der Teilnehmer Nicht-Spezialisten sein» sollten. ${ }^{45}$ Wie ergänzend ausgeführt wurde, waren einer Arbeitsgruppe zum Unterrichtsfach Deutsch demnach zu mindestens 30 Prozent fachfremde Lehrpersonen zuzuteilen.

Das Konzept gab eine nicht abgeschlossene Liste von Gebieten vor, um die Lehrplanarbeit zu strukturieren. Als erstes «Vorausgebiet» standen die Leitgedanken und Unterrichtsprinzipien der Realschule fest, als zweites «strukturelle Elemente», worunter das Zeugniswesen, Promotionen, Remotionen oder die Materialausstattung subsumiert waren. Als drittes Gebiet wurden die Fachgebiete aufgeführt. Das Konzept sah vor, in einer ersten Phase in fächerübergreifenden Themengruppen zusammenzuarbeiten, etwa Sprachfächer gemeinsam oder die naturkundlichen Fächer gemeinsam, um dann die «Entwicklung zum Teil neuer Fachgebiete» zu erwägen, bevor eine «Bestätigung, Konsolidierung der jetzigen Lehrpläne in einzelnen Fächern» anzugehen sei. ${ }^{46}$ Als viertes und fünftes Gebiet der Lehrplanarbeit waren im Konzept «Unterrichtsmethoden» und «Dokumentation von Lehr- und Lernmaterial» vorgesehen.

Für die Formulierung von allgemeinen Leitgedanken und Unterrichtsprinzipien in der ersten Arbeitsphase legte die Projektbeschreibung bereits Termine und einzuleitende Schritte fest. Alle in die Lehrplanarbeit involvierten Lehrpersonen sollten, noch bevor sie auf die einzelnen Gebiete aufgeteilt würden, an der Erarbeitung von Leitgedanken mitarbeiten. Ergänzend waren eine Befragung mittels Fragebogen vorgesehen sowie eine Orientierung an «Leitgedanken und Unterrichtsprinzipien anderer fortschrittlicher Schulen».47

Insgesamt beinhaltete dieses Konzept zur Reform der Realschullehrpläne im Kanton Basel-Stadt ab 1975 einen neuen Zugang in der Bestimmung schulischer Inhalte und zeigte zugleich auf, wie die Arbeit vorrangig durch Lehrpersonen organisiert werden könnte. Das Konzept war gewissermassen die Blaupause für die folgenden Lehrplanreformen. Indes handelte es sich nicht um die erste Intervention von Karl Frey in Reformprojekten des Kantons Basel-Stadt.

46 Lehrplanüberarbeitung der Realschule im Kanton Basel-Stadt, Projektbeschreibung, I0. 5. 1976, S. 3, Archivalien des ULEF. 
Auf Einladung des ULEF und der Sekundarlehrerkonferenz referierte Frey anlässlich der Reform des Lehrplans der Sekundarschulen im Januar 1974 über «Schwerpunkte der Lehrplanreform für die Sekundarschulen». Vorab führte er mit 20 Lehrpersonen, Inspektoren und Schulleitenden ein Interview und analysierte die provisorisch erlassenen Lehrpläne, um in seinem Referat praktische Vorschläge zur Veränderung der Unterrichtsinhalte und zu Arbeits- und Verfahrensweisen vorzulegen, wobei er bemerkte: «Selbstverständlich sind die Vorschläge mit durch ein curriculumtheoretisches Konzept beeinflusst, das in verschiedenen Veröffentlichungen dargelegt worden ist.» $4^{8}$ Er plädierte bezüglich der Unterrichtsinhalte für eine «Vermehrung des problemorientierten Unterrichts» oder die «Vereinigung kleiner Fächer zu Lernbereichen» und formulierte dann vier Vorschläge zur Arbeitsweise und zum Verfahren:49

I. Die Lehrpläne sind zu ergänzen durch Lernziele, methodische Hinweise und Lernkontrollen in einem allgemeinen Teil; in einem zweiten Teil sind die Lehrpläne zu ergänzen durch Modellskizzen, Modellentwürfe und Beispiele zur direkten Verwendung im Unterricht.

2. Die Lehrplankommission ist mit der Lehrerbildung zu koppeln: Dozenten einzelner Lehrbereiche wie auch der allgemeinen Didaktik sollen in den Prozess mit einbezogen werden. Eltern und weitere Interessierte sind ebenfalls zu involvieren.

3. Es bedarf der «Einrichtung eines ständigen Informationsaustausches». Über Entwürfe und Vorschläge, neue Lehrplaneinheiten oder Ideen ist frühzeitig zu informieren, wie etwa «mit der kleinen zweimonatigen Zeitschrift <Lehrplaninformation $>$ im Kt. Fribourg».

4. Lehrpersonen sind im Zuge steigender fachdidaktischer Anforderungen, beispielsweise durch problemorientierten Unterricht, zu entlasten. Sie sollen sich auf Lernbereiche konzentrieren und nicht mehr das gesamte Fächerspektrum unterrichten müssen.

Frey vermittelte durch seine Aktivitäten in den Lehrplanreformprojekten im Kanton Basel-Stadt Reformimpulse, die sowohl auf die Probleme bei der Erneuerung der schulischen Inhalte abzielten, als auch die Erneuerung der Verfahren der Lehrplanarbeit betrafen..$^{\circ}$ Das Vorgehen bei der Erarbeitung der Reformvorschläge entsprach seiner sozialwissenschaftlichen Arbeitsweise: Zur Vorabklärung führte er Gespräche mit bisher involvierten Personen und er er-

$4^{8}$ Karl Frey, Schwerpunkte der Lehrplanreform für die Sekundarschulen, 7. I. 1974, S. 2, Archivalien des ULEF.

49 Ebd., S. 3-5.

so Karl Frey war über die Lehrplanreformprojekte hinaus involviert bei Klausurtagungen zur Ausarbeitung von Leitideen für die Lehrerbildung oder anlässlich von Arbeitstagungen der ULEF-Kommission. Vgl. Protokolle der Arbeits- und Klausurtagungen, Archivalien des ULEF. 
hob Angaben in Umfragen. Die gewonnenen Informationen vermengte er mit

seinen Positionen aus der Curriculumforschung, für welche er aus seiner regen Publikationstätigkeit bekannt war. So wichen seine Reformvorschläge denn auch kaum von den gängigen Konzepten und Modellen der Curriculumforschung ab: die Reformen von zu entwickelnden Leitideen ausgehend entwickeln, Lernziele für Lehrplan und Unterrichtsvorbereitung formulieren und den Lehrplan durch eine Materialsammlung für den Unterricht ergänzen.

\subsubsection{Lehrplanreform im Kanton Basel-Stadt}

[...] so kann der neue Lehrplan als ein wesentlicher Beitrag zur inneren Schulreform betrachtet werden. Dabei darf festgestellt werden, dass der ursprüngliche Höhenflug bildungswissenschaftlicher Begrifflichkeit sich in der vorliegenden Endfassung auf zumindest erträgliche alltagsbezogene Sprache reduziert hat. ${ }^{S I}$

Der Vorsteher des Bildungsdepartements des Kantons Basel-Stadt machte I98 I in seiner Empfehlung an den Erziehungsrat, den neuen Lehrplan zu verabschieden, auf die ursprüngliche Absicht der bis dahin über zehn Jahre dauernden Lehrplanreformen aufmerksam: die Einführung der Koedukation auf der Sekundarstufe I. Zudem wies er subtil auf die in die Lehrplanarbeit eingeflochtenen Bezüge zur Curriculumforschung hin, deren sprachliche Herausforderungen aber scheinbar bewältigt werden konnten..$^{52}$

Wenngleich in den Basler Realschulen bereits ab i97I der erste Jahrgang von Schülerinnen und Schülern mehrheitlich gemeinsam unterrichtet wurde, dauerten die Lehrplanarbeiten bis I98 I und in bestimmten Teilbereichen bis weit darüber hinaus an. Währenddessen wandelte sich der ursprüngliche Lehrplan aus den I960er-Jahren zu einem umfassenden Dokument, welches über die einstigen Stoffsammlungen hinaus angereichert wurde mit allgemeinen Leitgedanken zur Schulstufe sowie übergeordneten Unterrichtsprinzipien und Lernzielen für die einzelnen Unterrichtsfächer. Insbesondere zwei Faktoren haben diese Entwicklung begünstigt: die Neupositionierung des ULEF durch dessen Leiter Guido Harder und eine neue Vorgehensweise bei der Ausarbeitung von Lehrplänen ab i975.

5I Arnold Schneider, Neuer Lehrplan für die Realschule, i 8. 8. I98 I, StABS, ED REG sa, S. 2.

$52 \mathrm{Zu}$ Verständnisproblemen von Lehrpersonen beim Vokabular der Curriculumliteratur vgl. Kapitel 4.3. 
In Basel-Stadt wurde im Gegensatz zu anderen Kantonen im untersuchten Zeitraum keine Bildungsplanungsstelle geschaffen. ${ }^{33}$ Diese Lücke verstand Harder mit dem ULEF weitgehend erfolgreich zu füllen - wenn auch nur bedingt über seine zehnjährige Amtszeit hinaus. Er positionierte sein Institut als zwischen der Wissenschaft, der Lehrerinnen- und Lehrerweiterbildung und den bildungspolitischen Instanzen vermittelnde Stelle. Er pflegte gute Kontakte zu wissenschaftlichen Experten in den für die Lehrplanreformprojekte relevanten Bereichen und engagierte sie zur Gestaltung von Werkstattseminaren und anderen Weiterbildungsveranstaltungen für Lehrpersonen. Auf diese Weise schuf Harder auch eine Plattform für Karl Frey und seine curriculumbasierten Vorschläge, wie eine Lehrplanreform nach den neusten Erkenntnissen der Wissenschaft zu realisieren sei. Die Analyse der Basler Lehrplanarbeiten bis 198 I hat anschaulich gezeigt, dass Freys Empfehlungen in der Konzeptarbeit umfassend berücksichtigt wurden, sowohl in Bezug auf die neu im Lehrplan zu konstituierenden Elemente als auch hinsichtlich der Vorgehensweise bei der Lehrplanarbeit.

I975 erklärten sich die Basler Lehrpersonen bereit zur aktiven Mitarbeit in der Erarbeitung eines neuen Lehrplanes und gelangten mit einem entsprechenden Antrag an den Erziehungsrat. Unter der Leitung von Harder und Frey wurde der Fokus weg von Diskussionen über die Stundentafeln hin zur Bestimmung schulischer Inhalte anhand übergeordneter Leitideen bewegt. In einem ersten Schritt wurden die allgemeinen Leitgedanken und Unterrichtsprinzipien ausgearbeitet, erst danach folgte die Formulierung von davon ableitbaren Unterrichtszielen. Allerdings führte die damit verknüpfte normative und daher nie abschliessend zu beantwortende Frage nach den Zielen von Schule und Bildung zu langwierigen Debatten und letztlich dazu, dass der Erziehungsrat des Kantons Basel-Stadt gerade bei diesem neuen Element des Lehrplans noch etliche Nachbesserungen forderte.

\subsection{Zentralschweizer Schulreformprojekte unter Anleitung des ZBS}

Im März 1971 trat der Kanton Luzern dem Schulkonkordat vom 29. Oktober 1970 bei und revidierte in diesem Zusammenhang das kantonale Erziehungsgesetz. Im Zuge dessen sollten im Volksschulbereich verschiedene, teilweise unterschiedlich motivierte Veränderungen eingeleitet werden, insbesondere die Verlängerung der obligatorischen Schulzeit auf neun Jahre. Dies nahm der

53 Für einen Überblick über die unterschiedlichen Entwicklungen, Aufgabenbereiche und Beziehungen von Bildungsplanungsstellen beziehungsweise pädagogischen Arbeitsstellen vgl. Bain und Roller, 200I. 
Kanton Luzern zum Anlass, um die Weiterentwicklung der Volkschuloberstufe grundlegend voranzutreiben. Aufgrund ähnlich gelagerter Reformabsichten spannten die Zentralschweizer Kantone in ihren Entwicklungsbemühungen bald zusammen und gründeten Mitte der i970er-Jahre den Zentralschweizer Beratungsdienst für Schulfragen (ZBS).

Das Ziel dieses Teilkapitels besteht darin, die vonseiten der Bildungspolitik initiierten Schulreformprojekte ab den I970er-Jahren zu beleuchten und erstens zu fragen, wie es dazu kommt, dass zunächst schulstrukturelle Veränderungsabsichten letztlich eine fundamentale Überarbeitung sämtlicher Volksschullehrpläne der Zentralschweizer Kantone initiierten. Es gilt ferner aufzuzeigen, nach welchen Vorstellungen und in Bezug auf welche Konzepte die neu zu konzipierenden Lehrpläne erarbeitet wurden, um die den Lehrplanreformen zugrunde liegenden Wissensbestände herauszuarbeiten. Zur Bearbeitung dieser Fragen wird vorrangig auf Berichte und Protokolle aus bildungspolitischen Kommissionen, auf Publikationen und Archivalien des ZBS und auf Regierungsratsberichte zurückgegriffen.

Die Ausführungen zu den Zentralschweizer Schulreformen folgen einem chronologischen Aufbau. Als Erstes werden die Reformbemühungen um die Volksschuloberstufe diskutiert, um dann zu zeigen, inwiefern Lehrpläne als wesentliches Reforminstrument lanciert wurden. Das dritte Unterkapitel ist der Entstehung und den Tätigkeiten des ZBS mit besonderer Berücksichtigung der ihm anvertrauten Lehrplanarbeit in der IEDK gewidmet. ${ }^{44}$

\subsubsection{Oberstufenreform in der Zentralschweiz}

Jede Schule hat ihren rechtmässig umschriebenen Schulkreis. Je länger je mehr müssen wir aber feststellen, dass Lehrer und Behörden bei ihren Problemen ausschliesslich an den geltenden Schulkreis und selten oder nie an eine Lösung denken, die vielleicht zwei benachbarten Schulkreisen gut dienen könnte.ss

54 Zur Innerschweizer Erziehungsdirektorenkonferenz (IEDK) gehören die Kantone Luzern, Uri, Schwyz, Obwalden, Nidwalden, Zug und der deutschsprachige Teil des Kantons Wallis. Sie bildet eine der drei deutschschweizerischen Regionalkonferenzen neben der Regionalkonferenz der Romandie und des Tessins. Regionalkonferenzen sollten zwei Ziele verfolgen: einerseits Geschäfte der EDK auf regionaler Ebene vorbereiten, andererseits regionenspezifische Geschäfte regeln. Weiterführend Lehmann, 2013, S. I 46.

55 Bericht des Regierungsrates des Kantons Luzern an den Grossen Rat über die gesamte Staatsverwaltung in den Jahren 1970 und 1971, S. 39, Forschungsbibliothek Pestalozzianum, LU HB 2. 
Unter dem Titel «Pädagogische und organisatorische Reformen» leitete das oben stehende Zitat im Regierungsratsbericht des Kantons Luzern für das Berichtsjahr 1970/7 I in denjenigen Berichtsabschnitt ein, welcher ohne Weiteres als Vorbote für die bald darauf einsetzende Reformkaskade im Volksschulbereich des Kantons Luzern und weiterer der IEDK zugehöriger Kantone gelesen werden könnte. So wurde beschrieben, dass für Schulen und Schulbehörden aktuell insbesondere zwei Probleme virulent seien: ein markanter Mangel an qualifizierten Lehrpersonen sowie schrumpfende Schülerzahlen in peripheren Kleinschulen.

Eine steigende Anzahl Lehramtskandidatinnen und -kandidaten und deren frühzeitiger Berufseinstig vermochten knapp die vielen, auch altersbedingten Austritte aus dem Schuldienst aufzuwiegen. Dennoch war «vielerorts ein qualitativ genügender Unterricht in Frage gestellt». ${ }^{6}$ Auf die anhaltende Abwanderung der Wohnbevölkerung aus Luzerner Rand- und Berggebieten musste mit der Aufhebung von Schulstandorten reagiert werden. ${ }^{57}$

Durch die Revision des kantonalen Erziehungsgesetzes am 4. Juli I97I wurde zudem Handlungsspielraum für Schulversuche geschaffen; besonders vom Modell der Gesamtschule erhoffte man sich, den Lehrermangel und die vergleichsweise tiefen Schülerzahlen in den peripheren Schulklassen auf einen Streich abwenden zu können. Bisher besuchten Schülerinnen und Schüler aus unterschiedlichen Schultypen der Sekundarstufe I - dazu zählen beispielsweise Realschulen und Oberschulen - nicht nur unterschiedliche Schulklassen, die Schulklassen waren meist auch in unterschiedlichen Schulhäusern untergebracht. Die Gesamtschulversuche sahen nun vor, mit der Zusammenlegung unterschiedlicher Schultypen erste Erfahrungen zu sammeln und «durch innere Differenzierung den individuellen Begabungen der Schülerinnen und Schüler gerecht zu werden».$^{8}$

Die Revision des Luzerner Erziehungsgesetzes im Juli 1971 ist überdies als unmittelbare Folge des Luzerner Beitritts zum Schulkonkordat einzuordnen. Das Erziehungsgesetz regelte die notwendigen gesetzlichen Anpassungen, welche die konkordatsbedingten Verschiebungen beim Schuleintrittsalter, bei der Schuldauer bis zur Matura sowie beim Schuljahresbeginn und - für den vorliegenden Zusammenhang besonders bedeutsam - eine Verlängerung der Schul-

56 Ebd., S. 37.

57 Allein für das Berichtsjahr I970/7I werden Schulschliessungen an den Standorten Doppleschwand, Hohenrain, Romoos, Marbach, Hasle, Escholzmatt, Mauensee und Hergiswil genannt.

58 Bericht des Regierungsrates des Kantons Luzern an den Grossen Rat über die gesamte Staatsverwaltung in den Jahren 1970 und I971, S. 39, Forschungsbibliothek Pestalozzianum, LU HB 2. 
pflicht auf neun Jahre erforderten. 59 In den kantonalen Richtlinien für die Regierungspolitik der Periode 1972-I975 war dementsprechend festgehalten: «Im Zusammenhang mit der Einführung des neunten obligatorischen Schuljahres wird eine Reform der Volksschul-Oberstufe angegangen. [...] Die angestrebte Differenzierung des Bildungsangebotes auf der Volksschul-Oberstufe verlangt grössere Schulzentren. Wenn auch der Landschaft die Vorteile eines qualitativ anspruchsvolleren und vielfältigeren Bildungsangebotes zugute kommen soll [sic], müssen vor allem die Oberstufenschulkreise vergrössert werden.» ${ }^{60}$ Insgesamt waren verschiedene schulstrukturelle Herausforderungen die Ursache dafür, eine Reorganisation der Volksschuloberstufe in die Wege zu leiten: $\mathrm{Zu}$ tiefe Schülerzahlen und kleinräumige Schulkreise auf der Oberstufe, ein Mangel an qualifizierten Lehrpersonen sowie die Erfordernis, die Schulzeit auf neun Jahre zu verlängern. Zusätzlich proklamierte der Regierungsrat eine «anzustrebende Chancengleichheit» und verwies zu deren Legitimation auf die «moderne Erziehungswissenschaft», welche «verlangt, dass die bisher allzu starren Schulstrukturen aufgelockert und die einzelnen Schulstufen möglichst durchlässig gestaltet werden». ${ }^{61}$

\subsubsection{Curriculumreform statt Lehrplanreform}

Um das vielschichtige Reformvorhaben der Volksschuloberstufe Luzern auszuarbeiten, ernannte der Erziehungsrat am I 2. Oktober 1972 die Kommission zur Gestaltung der Oberstufe. Unter den Kommissionsmitgliedern fanden sich Lehrpersonen aller Schultypen, Mitglieder aus den Schulinspektoraten, Rektorinnen und Rektoren, Delegierte aus den Erziehungsdepartementen und der Luzerner Schulplanung. Die Kommission wurde bewusst auch mit Vertreterinnen und Vertretern aus den Kantonen Nidwalden, Obwalden, Schwyz und Zug besetzt, zumal «ein erster Schritt in Richtung auf eine regionale Schulreform vollzogen werden» müsse. ${ }^{62}$

Allerdings bemühte sich die IEDK schon zu einem früheren Zeitpunkt um eine Sichtung von Lösungsmöglichkeiten angesichts der anstehenden Probleme auf der Oberstufe. Bereits am 6. Februar 1971 hat die IEDK zur Frage der «Cur-

59 Der Kanton Luzern ist dem Konkordat am I. 3. 197 I beigetreten. Zu Geschichte und Inhalt des Konkordats weiterführend Kapitel 3.I.

60 Richtlinien für die Regierungspolitik in den Jahren 1972-1975. In: Bericht des Regierungsrates des Kantons Luzern an den Grossen Rat, 22. 4. I974, S. 5, StALU, A 8 I 2 I67.

6I Bericht des Regierungsrates des Kantons Luzern an den Grossen Rat, 22. 4. I974, S. 5, StALU, A 8I 2 I67.

62 Die Orientierungsstufe in kooperativen Schulzentren. Bericht der Kommission zur Gestaltung der Oberstufe. Ende November 1973, StALU, A 8I 2 I67, S. 2. 
riculumreform der Sekundarschulen in der Innerschweiz» ein Gutachten bei der Freiburger Arbeitsgruppe für Lehrplanforschung in Auftrag gegeben und einen Monat später erhalten. ${ }^{63}$ Die im Gutachten formulierten Varianten und Ansätze für eine Reform der Sekundarstufe nahmen wesentliche Vorschläge vorweg, welche von der Kommission zur Gestaltung der Oberstufe kurz darauf ausgearbeitet wurden.

Der Vorsitz der Oberstufenkommission wurde Iwan Rickenbacher vom pädagogischen Institut der Universität Freiburg zugesprochen, weswegen die Kommission oftmals als «Kommission Rickenbacher» bezeichnet wurde. Neben der Mitarbeit bei der Freiburger Arbeitsgruppe für Lehrplanforschung (FAL) beschäftigte sich Rickenbacher bereits anlässlich seiner 1972 publizierten Promotionsarbeit mit ähnlichen Planungs- und Entwicklungsfragen. Am Beispiel des Kantons Schwyz erarbeitete er ab i 968 zuhanden der Kantonsregierung einen Entwicklungsplan für die Volksschuloberstufe, welcher auf die Zielvorgaben der EDK zu reagieren und «mit wissenschaftlicher Genauigkeit so komplexe Probleme wie die Anpassung des Schulwesens an die heutige Zeit» vorzunehmen hatte. ${ }^{64}$

Wie in den folgenden Ausführungen deutlich wird, orientierte sich die 1972 einberufene Kommission zur Gestaltung der Oberstufe stark an Befunden der Freiburger Forschungsarbeiten der FAL. Die Arbeiten der Kommission lieferten zudem das Grundgerüst für die bald darauf einsetzenden Schulreformprojekte im Kanton Luzern und in den übrigen Zentralschweizer Kantonen, wobei Lehrpläne als wesentliches Reforminstrument lanciert wurden.

Ende November 1973 legte die Kommission dem Luzerner Regierungsrat einen umfassenden Bericht vor, in welchem die politisch sensiblen Vorschläge zur Zusammenlegung einzelner Schultypen in Schulzentren einleitend umschrieben waren als Versuch, «das Wünschenswerte und das Mögliche in Einklang zu bringen».65 Wie die Grafik 9 zeigt, präsentierte der Kommissionsbericht die Zusammenführung der einzelnen Schultypen auf der Volksschuloberstufe unter dem neuen Begriff Orientierungsstufe. Organisatorisch blieben Spielräume für verschiedene Ausgestaltungen offen, welche sowohl Umsetzungen in lediglich administrativ gefassten Schulzentren erlaubten, wie auch Gesamtschulen zuliessen.

63 K. Aregger, K. Frey, U. Isenegger, Die Curriculumreform der Sekundarschulen in der Innerschweiz, März 1971, Archivalien der FAL.

64 Schulinspektor A. Kälin zum Planungsauftrag der Kantonsregierung Schwyz. In: Rickenbacher, I 972b, S. 43. Vgl. auch Kapitel 2.3.

65 Die Orientierungsstufe in kooperativen Schulzentren. Bericht der Kommission zur Gestaltung der Oberstufe, Ende November 1973, S. 3, StALU, A 8 I 2 I67. Hervorhebung im Original. 
Grafik 9: Die Orientierungsstufe in der Zentralschweizer Oberstufenreform

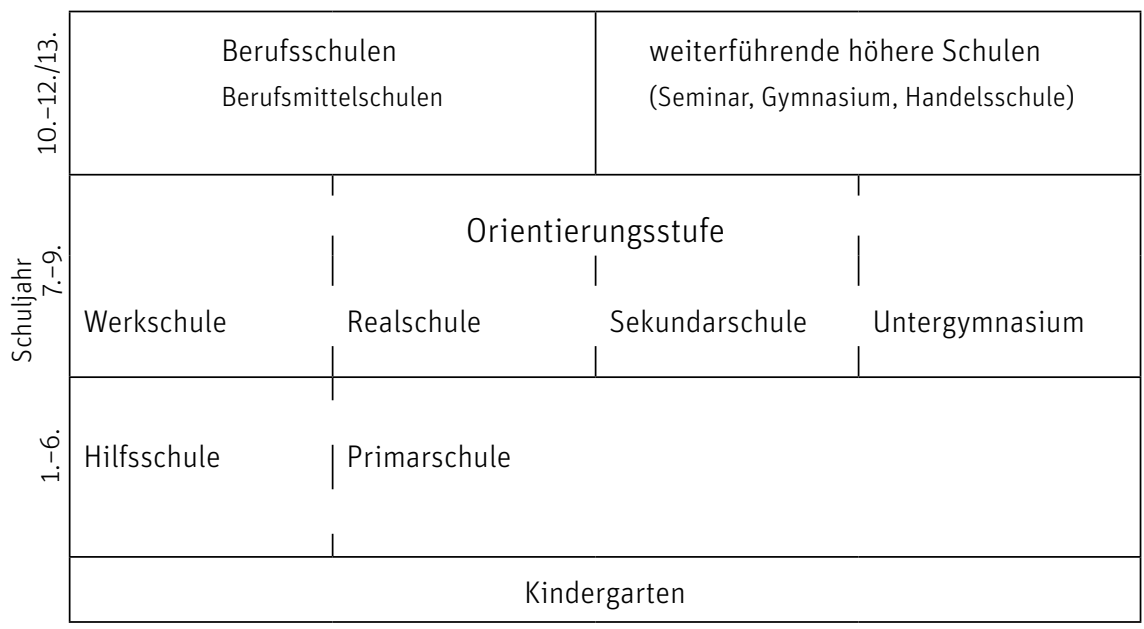

Quelle: Bericht der Kommission zur Gestaltung der Oberstufe, StALU, A 812 167, S. 6

Als Anknüpfungspunkte für die Argumentation zugunsten der Oberstufenreform waren im Bericht grundlegende «Zielsetzungen und Aufgaben der Schule» formuliert. Dazu ist vermerkt:

«Die Schule hat ganz allgemein

- die Lernfähigkeit der Schüler zu fördern

- die Lernziele immer wieder neu zu begründen

- die Lernenden zu motivieren

- Lernen zu belohnen [...]

- die Übertragung des Gelernten auf die Lebenssituation der Schüler zu ermöglichen (Heinrich Roth).» ${ }^{66}$

An der vorliegenden Setzung schulischer Ziele sind besonders der zweite und der fünfte Punkt bemerkenswert. Im Bericht war festgelegt, dass für schulisches Lernen neuerdings Lernziele zu formulieren seien, obwohl die im Erscheinungsjahr gültigen Lehrpläne des Kantons Luzern noch gar keine Lernziele aufwiesen. ${ }^{67}$ Darüber hinaus seien die Lernziele stets neu zu legitimieren.

66 Ebd.

67 Der Lehrplan für die Sekundarschulen des Kantons Luzern weist in der Ausgabe vom September 1972 wohl sehr allgemeine, übergeordnete Stoffziele und Bildungsziele aus, in den einzelnen Unterrichtsfächern kommen die «Stoffziele» allerdings Stoffsammlungen gleich. Ein Beispiel dafür aus der Algebra für die erste Klasse: «Grundbegriffe (Mengen, 
Diese Forderung knüpfte unmittelbar an die Position von Karl Frey und dessen Vorstellung von einer zirkulären, nie abgeschlossenen Überarbeitung von Curricula an. ${ }^{68}$ Auch die Referenz auf Heinrich Roth im fünften Punkt steht für die Rezeption aktueller Ansätze aus der Wissenschaft zur Fixierung schulischer Inhalte. Demgemäss sind Lernziele am Verwendungszweck der gegenwärtigen und künftigen Lebensbereiche der Schülerinnen und Schüler auszurichten. ${ }^{69}$

Der Kommissionsbericht knüpfte im Weiteren an die schulischen Zielsetzungen an und beinhaltete «Leitideen für die Reform der Orientierungsstufe». ${ }^{\circ} \mathrm{Sie}$ waren unterteilt in Leitideen zur «Schulorganisation», Leitideen zu «Selektion, Durchlässigkeit und Promotion», Leitideen zum «Lehrplan» sowie Leitideen zur «Lehrerbildung». Die folgenden Ausführungen konzentrieren sich auf ausgewählte Aspekte der Leitideen im Bereich der Lehrpläne. «Durch die Einführung des Begriffes Curriculum anstelle von Lehrplan soll die qualitative Verbesserung, die mit der Lehrplanreform bezweckt wird, herausgestellt werden. Künftige Curricula sollen neben allgemeinen Zielen der Schule und des Unterrichts mehr praxisbezogene Informationen (methodische Hinweise,

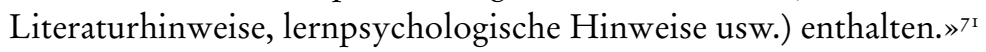

Das oben stehende Zitat aus dem Bericht der Kommission Rickenbacher verdeutlicht anschaulich den geplanten Wandel vom Lehrplan als Stoffsammlung hin zu einem nach curriculumtheoretischen Überlegungen angereicherten Dokument, welches neu als «Curriculum» zu bezeichnen sei. Für die Erarbeitung der neuen Curricula, so ist dem Bericht weiter zu entnehmen, «sind Arbeitsgruppen zu bilden, in denen Lehrer der betreffenden Lerngruppen mit weiteren Fachleuten (Erziehungswissenschafter, Psychologen, Fachdidaktiker, Berufsberater, Berufsleute, Eltern und Schüler) zusammenarbeiten». ${ }^{22}$ Weil, wie oben beschrieben, die zu formulierenden Lernziele einer permanenten Überprüfung in der Unterrichtspraxis unterzogen werden und $\mathrm{zu}$ entsprechenden Anpassungen führen müssten, legte der Bericht für die künftige Bewältigung der Lehrplanarbeit vier Empfehlungen nahe.

Erstens wurde eine «Ausbildung des Kaders für die Lehrplanarbeit»73 empfohlen. Damit meinte die Kommission eine Gruppe von Delegierten, welche

natürliche Zahlen, Grössen)». Lehrplan für die Sekundarschulen des Kantons Luzern, September 1972, S. 7, Forschungsbibliothek Pestalozzianum, LU GD i.

68 K. Frey, i969a. Vgl. dazu auch Kapitel 3.2.

69 Roth, 1968.

70 Die Orientierungsstufe in kooperativen Schulzentren. Bericht der Kommission zur Gestaltung der Oberstufe. Ende November 1973, S. 7 f., StALU, A 8 I 2 I67.

7I Ebd., S. 9. Hervorhebung im Original.

72 Ebd.

73 Ebd. 
sich strategisch der Lehrplanentwicklung annehmen und sich mit theoretischen Grundlagen beschäftigen würden. Zweitens sei die Entwicklung von Materialien für die Berufswahlorientierung sowie für die Förderung der Durchlässigkeit im Schulsystem anzustossen. Drittens sei in Anbetracht der nicht mehr aufrechtzuerhaltenden Annahmen über die Rollenverteilung zwischen den Geschlechtern der Hauswirtschaftsunterricht grundsätzlich neu zu gestalten. Viertens empfahl die Kommission in ihrem Bericht «die Einrichtung einer Beratungsstelle für Schulfragen in der Zentralschweiz».74 Die interkantonale $\mathrm{Zu}$ sammensetzung der Kommission habe gezeigt, dass in den anderen Kantonen der IEDK ähnliche Entwicklungsbedürfnisse und Prioritäten bestünden. In der Obliegenheit dieser Beratungsstelle wären dann beispielsweise Koordinationsarbeiten von Planungs- und Entwicklungsgruppen, Analysen im Schulbereich oder die Gewährleistung eines Informations- und Dokumentationsdienstes für Lehrpersonen.

Der Regierungsrat des Kantons Luzern hat den Kommissionsbericht zur Volksschuloberstufe am 22. April 1974 veröffentlicht. Das Kantonsparlament hat sich im darauffolgenden September zustimmend dazu geäussert.

Zusammenfassend zeigt der Bericht der Kommission zur Gestaltung der Oberstufe auf, wie der Luzerner Regierungsrat die drängenden Herausforderungen im Schulwesen des Kantons Luzern zu lösen plante. Infolge abwanderungsbedingter Schulschliessungen, Mangel an qualifiziertem Lehrpersonal und durch die Annahme des Schulkonkordats erzwungener, struktureller Veränderungen wurde eine umfassende Reform der Volksschuloberstufe erwogen, welche in «Orientierungsstufe» umbenannt wurde. Im selben Atemzug sollte mit der Realisierung von Schulzentren auf die Forderung nach mehr Durchlässigkeit zwischen den verschiedenen Schultypen im Sinne einer gesteigerten Chancengerechtigkeit reagiert werden.

Der Bericht macht zudem deutlich, dass die Zusammensetzung der Berichtskommission von grosser Bedeutung war. Mit der Vergabe des Vorsitzes an Iwan Rickenbacher erhielt die Kommission eine Leitung, welche in der Bearbeitung von entsprechenden Problemstellungen erfahren und mit den aktuellsten Erkenntnissen der Forschung zu bildungspolitischer Planung vertraut war. Es dürfte auch auf Rickenbachers Nähe zur Forschung der FAL an der Universität Freiburg und auf deren vorab verfasstes Gutachten zurückzuführen sein, dass die jüngsten Konzepte zur Curriculumkonstruktion unmittelbar in den Bericht eingeflossen sind. Wie noch deutlich werden wird, ermöglichte die Besetzung der Kommission mit Delegierten aus weiteren Innerschweizer Kantonen auch eine Intensivierung der Kooperation innerhalb der IEDK. 
Letztlich führte die vertiefte Auseinandersetzung mit der Volksschuloberstufe dazu, dass über die Aufgaben und Zielsetzungen der Schule innerhalb der neu zu gestaltenden Orientierungsstufe nachgedacht wurde. So setzte die Kommission die schulischen Inhalte mit auf die Reformagenda und brachte die Auseinandersetzung mit Lehrplänen, deren Funktion und Elementen wie auch die Art und Weise der Aufbereitung schulischer Inhalte in Gang. Die Diskussion über die Erarbeitung von Curricula nach aktuellen, in der Curriculumforschung verorteten Ansätzen war lanciert, erste Vorschläge zum Vorgehen lagen vor, die Schaffung eines schulischen Beratungsdienstes schien angesichts der Fülle von anstehenden Reformprojekten höchst angezeigt.

\subsubsection{Curriculumreform unter Anleitung des ZBS}

Bereits im Mai 1974, einen Monat nach der Publikation des Kommissionsberichtes zur Gestaltung der Oberstufe, unterzeichnete der Präsident der IEDK den Beschluss über «Planung und Koordination der Reform der Volksschuloberstufe der Zentralschweiz».75 Das Ziel bestand laut dem Beschluss darin, die Entwicklungen im Zentralschweizer Schulwesen «aufeinander abzustimmen», wobei zwei Bereiche vordringlich seien, «die Erarbeitung neuer Lehrpläne und die Entwicklung eines Konzepts für die Lehrerausbildung». Die Entwicklungsarbeiten müssten zudem «speditiv» und «wissenschaftlich abgestützt» vorgenommen werden und sie seien «nicht bloss durch nebenamtliche Kommissionsarbeit zu leisten», weshalb ein Planungs- und Beratungsdienst für die Kantone der IEDK einzurichten sei. ${ }^{76}$

Diesem IEDK-Beschluss war eine Serie von Kommissions- und Ausschusssitzungen zur Positionierung und Finanzierung eines neuen Beratungsdienstes innerhalb der IEDK vorausgegangen, wobei einige Entwürfe und Planungsdokumente bereits bestehender, ähnlicher Beratungsdienste in anderen Kantonen als Vorlage dienten. So befinden sich unter den Archivalien zur Gründung des ZBS auch die Statuten des Institut de recherche et de documentation pédagogigque (IRDP) und der Bildungsplanung des Kantons Zürich. Die IEDK hat sich insbesondere aufgrund der interkantonalen Ausrichtung des zu gründenden Planungsdienstes stark am IRDP orientiert. ${ }^{77}$

75 Beschluss über Planung und Koordination der Reform der Volksschuloberstufe der Zentralschweiz, 22. 5. 1974, StALU, A 69638 I.

76 Ebd., S. I.

77 Vgl. Protokolle der Kommission zur Gestaltung der Oberstufenreform und Protokolle des Ausschusses der Kommission, StALU, A 696 38r. Vgl. auch Protokolle zur Gründung des ZBS, StALU, A 696380. 
Parallel zu den Gründungsbestrebungen des ZBS fanden erste Orientierungsveranstaltungen für Lehrpersonen statt, welche unmittelbar von den anstehenden Veränderungen anlässlich der Oberstufenreform betroffen waren. Ende Mai und Anfang Juni 1974 stimmten dann die Regierungsräte der beteiligten Kantone Uri, Schwyz, Nidwalden, Obwalden, Luzern und Zug der vorgelegten Fassung zur Errichtung eines Planungs- und Beratungsdienstes zu.

\section{Der ZBS nimmt seine Tätigkeit auf}

Per I. Oktober 1974 nahm der neu gegründete Zentralschweizer Beratungsdienst für Schulfragen (ZBS) seine Arbeit auf. Er wurde als IEDK-Stabsstelle konzipiert, welche, vorerst befristet auf fünf Jahre, die Einführung der Orientierungsstufe unterstützen sollte. Im Jahresbericht der EDK wurden die Gründung und der künftige Aufgabenbereich des ZBS wie folgt umrissen: Es sei sinnvoll, wenn die anstehenden Planungsarbeiten «koordiniert, andererseits aber auch rationalisiert» würden, zumal es in der Innerschweiz sowohl an einem pädagogischen Institut wie auch an «Erziehungswissenschaftern» mangle. ${ }^{78}$ Die bestehenden und neu zu bildenden Arbeitsgruppen seien «in ihren Bestrebungen wissenschaftlich zu beraten», etwa mittels «Einführungskursen z. B. in die Lehrplanarbeit, Sammeln der notwendigen Unterlagen und Informationen, Herstellen wichtiger Querverbindungen, durch Erarbeiten von Planungsdispositionen, Redigieren der entstehenden Arbeitspapiere usw.».79

Obschon der Aufgabenkatalog des ZBS zunächst wenig abschliessend festgelegt war, wollte die IEDK auf jeden Fall etwaige Befürchtungen, es könnte ein interkantonales Planungsbüro entstehen, zerstreuen, welches in die Schulhoheit der beteiligten Kantone eingreifen würde. Vielmehr sollte der Beratungsdienst «die zum Teil stecken gebliebene Arbeit wieder in Gang bringen», Unterstützung «bei der Formulierung von Lernzielen» anbieten und also nicht selbst Lehrpläne entwickeln, sondern «Hilfeleistungen zu deren Konstruktion» erbringen sowie Lehrpersonen in der Ausarbeitung und Einführung «des neuen Instrumentes» unterstützen. ${ }^{8 \circ}$

Im Januar 1977 blickte der ZBS erstmals auf seine Anfänge zurück und publizierte den «Grundlagen- und Tätigkeitsbericht I974-I976», welcher sowohl die Funktion der Rechenschaftslegung gegenüber der IEDK als Trägerschaft übernahm, wie auch als Informationsorgan für eine breite Öffentlichkeit gedacht war. ${ }^{81}$ Iwan Rickenbacher hatte inzwischen die Leitung des Beratungsdienstes abgegeben und wurde zum Direktor des Lehrerseminars in Rickenbach im

78 Jahresbericht der Konferenz der kantonalen Erziehungsdirektoren 1973/74, S. 4I.

79 Ebd.

80 Ebd., S. 4I f.

8 I Grundlagen und Tätigkeitsbericht 1974-I976, ZBS, I I. I. I977, StALU, A 810 4I9. 
Kanton Schwyz berufen. Seine Nachfolge angetreten und die Aufbauarbeit des ZBS weitergeführt hatte Anton Strittmatter, ebenfalls ein ehemaliges Mitglied der Freiburger Arbeitsgruppe. ${ }^{{ }^{2}}$ Mit Bezug auf das Statut des ZBS betonte Strittmatter im Tätigkeitsbericht erneut die Schwerpunkte der bisherigen und zukünftigen Aufgaben des ZBS, namentlich die Realisierung der Reform der Volksschuloberstufe der IEDK..$^{83}$

Legitimiert durch die Leitideen für die künftige Orientierungsstufe legte der ZBS den Schwerpunkt seiner Tätigkeiten von Beginn weg auf den Bereich der Lehrerinnen- und Lehrerbildung sowie die Entwicklung eines Rahmenlehrplans in der IEDK. Man ging davon aus, dass mit einem Rahmenlehrplan die Förderung von Durchlässigkeit im Schulwesen und die Fixierung eines gemeinsamen Kerns an schulischen Inhalten realisiert werden könnte. Für die Realschulen, die Sekundarschulen und die Untergymnasien war beabsichtigt, spezifische Vorstellungen von Bildungszielen und Bildungsinhalten herauszuarbeiten und gegebenenfalls mit bestehenden Richtlinien abzugleichen. Es war ferner vorgesehen, zunächst eine Stundentafel für die künftige Orientierungsstufe auszuarbeiten. Die Stundentafel galt als «äussere[r] Rahmen, der die innere Reform der Orientierungsstufe erst ermöglicht». ${ }^{8}{ }^{8}$ Weder die Arbeiten zur Stundentafel noch die inhaltlichen Arbeiten zu den Bildungszielen waren bis zum Berichtsjahr 1977 abgeschlossen.

Hingegen vermeldete der «Grundlagen- und Tätigkeitsbericht 1974-1976» die nun einsetzende Vernehmlassung und Erprobung von jüngst entwickelten Lehrplänen für die Sekundarschulen. Es handelte sich dabei allerdings um Lehrpläne, deren Entwicklung die IEDK bereits I97I einer interkantonalen Kommission übertragen hatte. Als bei der Aufgabenformulierung des ZBS von inskünftig zu leistender Unterstützung bei festgefahrenen Kommissionsarbeiten die Rede war, war implizit auch der Entwicklungsprozess dieses Sekundarlehrplanes gemeint. Entsprechend verhalten äusserte sich Anton Strittmatter im ZBS-Bericht über das vorgelegte Endprodukt und schlug vor zu prüfen, «ob entweder Lehrplanteile mit kurzfristig leistbaren Änderungen übernommen

82 In den Akten des ZBS zur Nachfolge Iwan Rickenbachers finden sich ausschliesslich Dossiers von Mitgliedern der FAL. Dies lässt zwei Schlüsse zu: ı. Die FAL-Mitglieder waren die einzigen qualifizierten Wissenschaftler für die vorgesehene Aufgabe auf dem deutschsprachigen Schweizer Arbeitsmarkt. 2. Die IEDK wollte bevorzugt Personal der FAL anwerben, weil es sich gerade mit Projekten in der IEDK und Publikationen bereits einen Namen gemacht hatte. Vgl. Walter Gut, i 5. 5. I975, StALU, A 810 4 I 3.

83 Die Aufsichtskommission des ZBS hatte sich dementsprechend bei der Suche nach einer Nachfolge für die ZBS-Leitung explizit gegen eine Person ausgesprochen, welche den ZBS in Richtung einer Schulforschungsstelle hin weiterentwickeln wollte. 3. Protokoll der Aufsichtskommission, Mai 1975, StALU, A 696382.

84 Grundlagen und Tätigkeitsbericht 1974-1976, ZBS, I I. I. I977, S. 28, StALU, A 8 I0 4 I 9. 
werden können, einer gründlichen und längere Zeit beanspruchenden Überarbeitung bedürfen oder aber pauschal abgelehnt werden und völlig überarbeitet werden müssten». ${ }^{85}$ Insgesamt schien der vorgelegte Sekundarlehrplan aus der Zeit vor der Gründung des ZBS nicht den jüngsten Standards, Erwartungen und Bedürfnissen zu entsprechen.

Besonders vage blieb die Berichterstattung des ZBS im Januar I $977 \mathrm{zu}$ den Bestrebungen im Bereich der Lehrerinnen- und Lehrerbildung für die Orientierungsstufe. Der ZBS setzte voraus, dass die Entwicklung und die Umsetzung von Neuerungen wie etwa von Lehrplänen und Lehrmitteln nur unter Mitwirkung der Lehrpersonen möglich seien. Die Lehrpersonen für die Sekundarstufe I wie für die Kantonsschulen wurden bislang aber nicht in Kantonen der IEDK ausgebildet, weshalb es von Beginn weg klar gewesen sei, dass «die Schaffung einer zentralschweizerischen Einrichtung zur Ausbildung aller Orientierungsstufenlehrer eine dringliche Konsequenz aus der Einleitung der Orientierungsstufenrefom darstellt». ${ }^{86}$ Die Erstellung der «Reformfähigkeit» der Lehrpersonen war aus Sicht des ZBS demnach nicht gegeben, zumal die Fortbildung der Lehrpersonen allein für diese Aufgabe nicht ausreichend sein würde und deshalb ein Antrag an die IEDK für die Gründung einer entsprechenden Hochschule in Luzern erfolgen sollte. ${ }^{87}$

\section{Entwicklungsgrundsätze für den Orientierungsstufen-Rahmenlehrplan}

Im Februar 1978 verkündete der ZBS, dass die Entwicklungsarbeiten am Rahmenlehrplan für die Orientierungsstufen starten können, die IEDK habe an ihrer Sitzung vom ıo. Februar den entsprechenden Projektaufriss genehmigt. Die anstehenden Arbeiten wurden in zwei Phasen aufgeteilt. Als Erstes waren die Lehrpläne von vier Fachbereichen auszuarbeiten: ${ }^{88}$ I. Deutsch, 2. Mathematik, 3. Naturlehre: Biologie, Chemie, Geografie, Physik, 4. Gemeinschaftserziehung, Lebenskunde, Geschichte.

Der Start für die zweite Serie der Lehrplanarbeiten war für Herbst 1978 vorgesehen; die Fachbereiche wurden noch nicht genannt. Wie in den Entwicklungen der Schulreformprojekte im Kanton Basel-Stadt zeichnete sich auch in der Zentralschweizer Lehrplanarbeit die Tendenz ab, einzelne Fächer gemäss den Empfehlungen von Karl Frey zu Fachgruppen zusammenzufassen. ${ }^{89}$ Die

85 Ebd.

86 Ebd., S. 36.

87 Für den weiteren Verlauf der Hochschulgründung in der Zentralschweiz vgl. Huber, 2017 .

88 Start der Lehrplanarbeiten am Orientierungsstufen-Rahmenlehrplan, Kurzorientierung, o. D., StALU, A 888 295.

89 Vgl. Kapitel 4.I.4 zur Rolle von Karl Frey in den Reformprojekten des Kantons BaselStadt. 
Kurzorientierung des ZBS zum Startschuss der IEDK ist aber insbesondere aufgrund des folgenden Zusatzes interessant: «Die Arbeitsweise in der Projektleitung (ZBS) und in den Kommissionen richtet sich nach den Verfahrensgrundsätzen, wie sie von den Teilnehmern des Kaderkurses «Lehrplanentwicklung» (Winter 77/78) erarbeitet wurden. Die Grundsätze werden demnächst an geeigneter Stelle veröffentlicht.»90

In der elften Ausgabe der «Schweizer Schule» von 1978 publizierte der Leiter des ZBS, Anton Strittmatter, die für die Lehrplanarbeiten der Folgejahre gültigen Grundsätze. ${ }^{91}$ Die Grundsätze gliedern sich in fünf Abschnitte, welche im Folgenden überblicksartig zusammengefasst werden; anschliessend wurden sie in Bezug auf die Provenienz ihr inhärenter Konzepte diskutiert. Generell verkörpern die Grundsätze der Lehrplanarbeit eine strategisch-programmatische Positionierung und Vorgabe dafür, wie unter wissenschaftlicher Anleitung Ziele und Inhalte der künftigen Orientierungsstufe festzulegen sind. Der erste Grundsatz lautete zusammengefasst:

I. Die Lehrpläne enthalten die folgenden Elemente: Leitideen, Einstellungsziele, Jahresziele, Stoffverteilungspläne sowie zusätzliche didaktisch-methodische Hilfen für Lehrpersonen.

Die am Kaderkurs Teilnehmenden haben sich entschieden, mit der Berücksichtigung der genannten Elemente alle entsprechenden Anliegen seitens der Curriculumforschung aufzunehmen. ${ }^{92}$ Zusätzlich zu den traditionell gängigen Stoffverteilungsplänen würden, wie in den präzisierenden Ausführungen deutlich gemacht wurde, insbesondere anhand der Einstellungsziele und Jahresziele Elemente «zur Überprüfung des Lernerfolgs bzw. von Fortschritten im Lernprozess» der Schülerinnen und Schüler in den Lehrplan eingeführt. ${ }^{93}$ Darüber hinaus machte der Ruf nach Leitideen eine Auseinandersetzung über die spezifischen Bildungs- und Erziehungsziele dieser Schulstufe notwendig.

2. Die Lehrplanentwicklung dauert pro Fachbereich zwei Jahre und erfolgt in vier Phasen: r. Fortbildung der Kommissionsmitglieder, 2. Entwicklungsarbeit, 3. Erprobung, 4. Einführung und Inkraftsetzung.

In diesem Abschnitt formulierten die Teilnehmenden die Grundsätze für das idealtypische Verfahren der Lehrplanentwicklung. Wie sich noch herausstellen sollte, war die Entwicklungsdauer von zwei Jahren pro Fachbereichslehrplan etwas optimistisch angesetzt. ${ }^{94}$ Bezeichnenderweise sah das Verfahren vor, zu-

90 Start der Lehrplanarbeiten am Orientierungsstufen-Rahmenlehrplan, Kurzorientierung, o. D., StALU, A 888 295, S. I.

9I Strittmatter, I978b.

92 Vgl. dazu beispielsweise K. Frey, i969a.

93 Strittmatter, 1978 b, S. 380.

94 Der verbindliche Lehrplan für die Orientierungsstufe der IEDK-Kantone ist erst 1986 erschienen. 
nächst alle Involvierten in Fortbildungen mit den neuen Konzepten der Lehrplanarbeit vertraut zu machen, was unmittelbar mit der gewünschten Zusammensetzung der Lehrplankommissionen zusammenhängt. Dazu der folgende Grundsatz:

3. An der Lehrplanentwicklung sind zu beteiligen: die Lehrerschaft aller Schulstufen, Forschende aus den Bildungs- und Fachwissenschaften und der ZBS zur Auswahl und Nennung ebensolcher wissenschaftlicher Experten als Beraterinnen und Berater. Besonders bei der Entwicklung von Leitideen sind die Eltern, (Bildungs-)Politiker, Schülerinnen und Schüler, Vertreterinnen und Vertreter aus Wirtschaft und Kirche einzubeziehen.

Die möglichst breit angedachte Abstützung der Lehrplanarbeit durch eine vielfältige Zusammensetzung der Lehrplankommissionen verdeutlicht zwei Anliegen. Einerseits sollte in demokratischer Aushandlung und durch immer wieder eingeschaltete Vernehmlassungen und Rückmeldeschlaufen ein grösstmöglicher Konsens über die Bildungs- und Erziehungsziele der Schule angestrebt werden. Andererseits wurde dem ZBS eingeräumt, geeignete «Pädagogen, Psychologen und Soziologen» sowie Experten aus den Fachwissenschaften zur Begleitung der Lehrplankonstruktionsprozesse auszuwählen.9s

4. Ein transparentes «Informationssystem» fördert anhand von Informationsveranstaltungen oder einer eigenen Projektzeitung den gegenseitigen Austausch im Lehrplanprozess. ${ }^{96}$ Der ZBS gewährleistet den Austausch zwischen parallel laufenden Reformvorhaben. Notwendige Informationen werden bei Fachleuten, aus der fachdidaktischen Literatur oder Lehrmitteln gewonnen.

Auch dieser Grundsatz schrieb dem ZBS eine zentrale Funktion im Prozess der Konstruktion von Lehrplänen zu. Wenn die Arbeit gleichzeitig in vier verschiedenen Fachbereichen zu erfolgen hatte, bedurfte es einer koordinierenden Stelle, welche den Überblick über die laufenden Projekte und den gegenseitigen Austausch gewährleisten würde. Der Wille zur grösstmöglichen Transparenz der Erarbeitungsprozesse machte ein Informationsmedium erforderlich. Ein solches veröffentlichte der ZBS ab 1978 regelmässig unter dem Titel «ZBSInformationen». Das Ziel bestand in der «Verbesserung der öffentlichen Kontrolle unseres Tuns, der Mitsprachebedingungen für die Lehrerschaft und des Zusammenspiels der Arbeiten in den verschiedenen Teilprojekten».97

5. Die Erziehungsbehörden entscheiden über Leitideen oder Ziele. In Lehrplankommissionen und zugehörigen Dokumenten ist die Sprache einfach und verständlich zu halten.

95 Strittmatter, 1978b, S. $38 \mathrm{I}$.

96 Ebd.

97 ZBS-Informationen, Zentralschweizer Beratungsdienst für Schulfragen, I8. 7. I978, S. I, StALU, A 696382. 
In diesem letzten Grundsatz wurde die Kompetenz der Entscheidung über die Kernelemente der entstehenden Lehrpläne - dazu gehörten Leitideen, Einstellungsziele und Jahreslernziele - den Erziehungsbehörden eingeräumt. Dennoch behielt man sich in den Präzisierungen vor, dass Lehrpläne zusätzliche «Teile rein anregender, vorschlagender Natur enthalten, welche nicht Gegenstand der behördlichen Entscheidung im Sinne der Verbindlich-Erklärung sind».9 ${ }^{8}$ Damit waren Stoffverteilungspläne und zusätzliche didaktisch-methodische Hilfen für Lehrpersonen gemeint.

Werden die unter Anleitung des ZBS entstandenen fünf Grundsätze aus dem Kaderkurs «Lehrplanentwicklung» vor dem Hintergrund der I97 I publizierten Thesen der FAL zu Bildungsplanung und Schulreform ${ }^{99}$ betrachtet, zeigen sich frappierende Parallelen. Damals postulierte die FAL, Schulreform sei als Curriculumreform zu realisieren und Curriculumreform bedinge die Einführung überprüfbarer Lernziele. Die FAL vertrat ferner die These, wonach Schulreform partizipativ zu erfolgen habe und gesellschaftlich möglichst breit abgestützt zu organisieren sei. Darüber hinaus handle es sich um eine permanente Aufgabe, welche unter Zuhilfenahme und Kontrolle von wissenschaftlicher Beratung zu erfolgen habe. Alle genannten Thesen der FAL finden sich sinngemäss in den oben diskutierten Grundsätzen zur Lehrplanarbeit für die IEDK wieder. Die einzige Abweichung besteht darin, dass die FAL I97 I noch die These vertrat, dass den Schulinspektoren ${ }^{100}$ eine bedeutende Rolle in der Informationsverbreitung zukomme. Diese Funktion hat in der Programmatik der Zentralschweizer Orientierungsstufe-Lehrplanarbeit der neu geschaffene ZBS übernommen.

\section{Entwicklungsarbeiten im Lehrplanwesen der IEDK}

Bis Mitte der I980er-Jahre war die Berichterstattung des ZBS in seinem Informationsblatt geprägt von Darstellungen zu den laufenden Lehrplanarbeiten. Zusätzlich zu den Entwicklungsarbeiten in den Fachkommissionen für den Lehrplan der Orientierungsstufe rückte neu auch die Lehrplanarbeit für die Primarschulen in den Fokus. ${ }^{\text {Ior }}$ Die IEDK hatte hierzu per Anfang I979 das Mandat des ZBS erweitert. Neu konnte der ZBS die gesamte Oberstufenzeit zu seinem Aufgabenbereich zählen und ist aus diesem Grund mit weiteren Mitteln zur Anstellung von zusätzlichem Personal ausgestattet worden. ${ }^{102}$

98 Strittmatter, 1978 b, S. 382.

99 Vgl. dazu Kapitel 3.2.3 sowie Freiburger Arbeitsgruppe für Lehrplanforschung, I97 I.

ı०० In der IEDK war im Untersuchungszeitraum ausschliesslich männliches Personal in der Schulinspektion angestellt.

IоI Vgl. das Kapitel 4.4.

I02 ZBS-Informationen, Zentralschweizer Beratungsdienst für Schulfragen, I8.7. 1978, StALU, A 696382. 
Wenngleich der ZBS um grösstmögliche Transparenz und Übersicht über die laufenden Projekte bemüht war, herrschte grosse Unübersichtlichkeit in der Frage, an welchen Lehrplänen sich derzeit sowohl die Schulpraxis wie auch die Ausbildung der Lehrerinnen und Lehrer zu orientieren habe. In den Primarschulen etwa kursierten nach wie vor gültige Ausgaben aus den i970er-Jahren, welche mittlerweile vergriffen waren. Auf einen Neudruck wollte der Erziehungsrat des Kantons Luzern aber verzichten, weil ein den neuen Konzepten gemässer Lehrplan in Ausarbeitung war. Der Luzerner Erziehungsrat behalf sich wie folgt: «Für die Übergangszeit soll deshalb erneut, wie bereits vor zwei Jahren, eine Fassung herausgegeben werden, aus der ersichtlich ist, was wo zu finden ist. Die neue Ausgabe ist deshalb im Untertitel als «Orientierungshilfe für den Lehrer> zu bezeichnen.» ${ }^{103}$

Die Berichterstattung in den Informationsbulletins des ZBS über die laufenden Lehrplanverfahren zeigt deutlich, dass die 1978 entworfenen Grundsätze für die Lehrplanarbeiten nicht bloss geschriebenes Wort geblieben waren. Entsprechend den festgelegten Grundsätzen durchliefen die ersten Entwürfe aus den Fachkommissionen zunächst eine Vernehmlassung bei Behörden und Lehrpersonen in den beteiligten Kantonen. Anschliessend folgte eine zwei- bis dreijährige Erprobungsphase, bevor die Fachlehrpläne nach letzten Anpassungen von den beteiligten Kantonen als definitiv erklärt und gedruckt wurden. ${ }^{104} \mathrm{Nicht}$ nur auf Verfahrensebene, auch in Bezug auf den inhaltlichen Aufbau und die Strukturierung der neuen Lehrpläne erfolgte die Umsetzung sehr nahe an den Grundsätzen. Bereits in der ZBS-Berichterstattung im Mai 198 I über den derzeitigen Entwicklungsstand in den einzelnen Kommissionen war zu lesen: «Alle Lehrpläne werden nach einem einheitlichen Schema aufgebaut. Der Teil A «Leitideen und Richtzieles enthält die Kapitel (a) Allgemeine Bildungsziele, (b) Erläuterungen/Begründungen, (c) Rahmenbedingungen, (d) didaktische Prinzipien und (e) Richtziele. Der Teil B «Grobzieles ist in die Spalten (I) Grobziele, (2) nähere thematische Bestimmungen, (3) methodische Hinweise/Hinweise zu Lehrmitteln und (4) Querverbindungen, gegliedert.» ${ }^{\text {105 }}$

Passend zu diesen Ausführungen wurde eine Illustration über die einzelnen Fachbereiche und ihre Teilbereiche abgedruckt, um mit einer Schraffur über den derzeitigen Entwicklungsstand zu informieren.

Unter dem Titel «Die Einführung neuer Lehrpläne» war dann im November I985 in einem Beitrag in «ZBS-Information» zu lesen, dass nach einer zehnjäh-

I03 Protokoll des Erziehungsrates des Kantons Luzern, 7. 5. 1986, StALU, A I 2539.

I04 ZBS-Information. Zentralschweizer Beratungsdienst für Schulfragen, Oktober 1984, S. Io, StALU, A 696382.

I05 ZBS-Information. Zentralschweizer Beratungsdienst für Schulfragen, I5. 5. I98 I, S. 4, StALU, A 696382. 
rigen Phase der Erarbeitung von Lehrplänen für die Orientierungsstufe und die Primarstufe es nun gelte, anhand einer «vertieften Einführungsweise [...] jeder Lehrkraft den Weg ins «Neuland soweit zu ebnen, dass sie sich im Lehrplan und im Lehrmittel auskennt». ${ }^{106}$ Über die bereits an der Ausarbeitung beteiligten Lehrpersonen hinaus seien jetzt die kantonalen Stellen für die Fortbildung der Lehrpersonen, die im ZBS und in den kantonalen Erziehungsdepartementen Mitarbeitenden zur aktiven Mitarbeit aufgerufen.

Am Primarschullehrplan für den Fachbereich «Mensch und Umwelt» vom November 1983 soll zum Schluss dieses Teilkapitels deutlich gemacht werden: Die Grundsätze zur Entwicklung des Lehrplans für die Orientierungsstufe wurden auch in der Überarbeitung der Lehrpläne für die Primarschule angewendet. Die Grafik ı zeigt anschaulich das Modell, wonach aus Leitideen und Bildungszielen Grobziele abgeleitet wurden, welche wiederum als Grundlage für die Formulierung von inhaltlichen und instrumentellen Zielen in einzelnen Themenbereichen herangezogen wurden. Daran anschliessend beinhaltete der Lehrplan neuerdings auch Vorschläge für Unterrichtseinheiten.

Mit Blick auf die Ausführungen zum Jahrbuch der EDK und dessen Vorschlägen betreffend Minimalanforderungen an die Form von Lehrplänen ${ }^{107}$ wird hier besonders deutlich, dass die Lehrplanarbeit in der IEDK sich unmittelbar an diesen Empfehlungen orientierte und sie ohne Abwandlung bei der Konzeption der eigenen Lehrpläne implementierte.

\subsubsection{Lehrplanreform in der IEDK}

Es liegt in der Natur unserer Arbeit, dass grosse Ereignisse und Erfolge sehr selten, Misserfolge und Kritik häufig und stete und eher unauffällige Entwicklungsarbeit in Arbeitsgruppen und an Konferenzen und Kursen die Regel sind. ${ }^{\text {os }}$

Mit diesen Worten führte der Leiter des ZBS, Anton Strittmatter, in den Jahresrückblick von 1978 ein. Er offerierte der Leserschaft der «ZBS-Information» des seine Sicht auf die geleisteten Arbeiten jenes Beratungsdienstes, welcher eigens zur Begleitung, Beratung und Organisation der Zentralschweizer Oberstufenreform geschaffen wurde.

Während der hier analysierten Untersuchungsperiode von 1970 bis in die frühen I980er-Jahre zeichnete sich ein Wandel im Verständnis und in der Konzep-

Io6 Ebd., S. 5 f.

I07 Vgl. dazu Kapitel 3.3.

I08 ZBS-Informationen, Zentralschweizer Beratungsdienst für Schulfragen, I 5. 3. 1979, S. I, StALU, A 696382. 


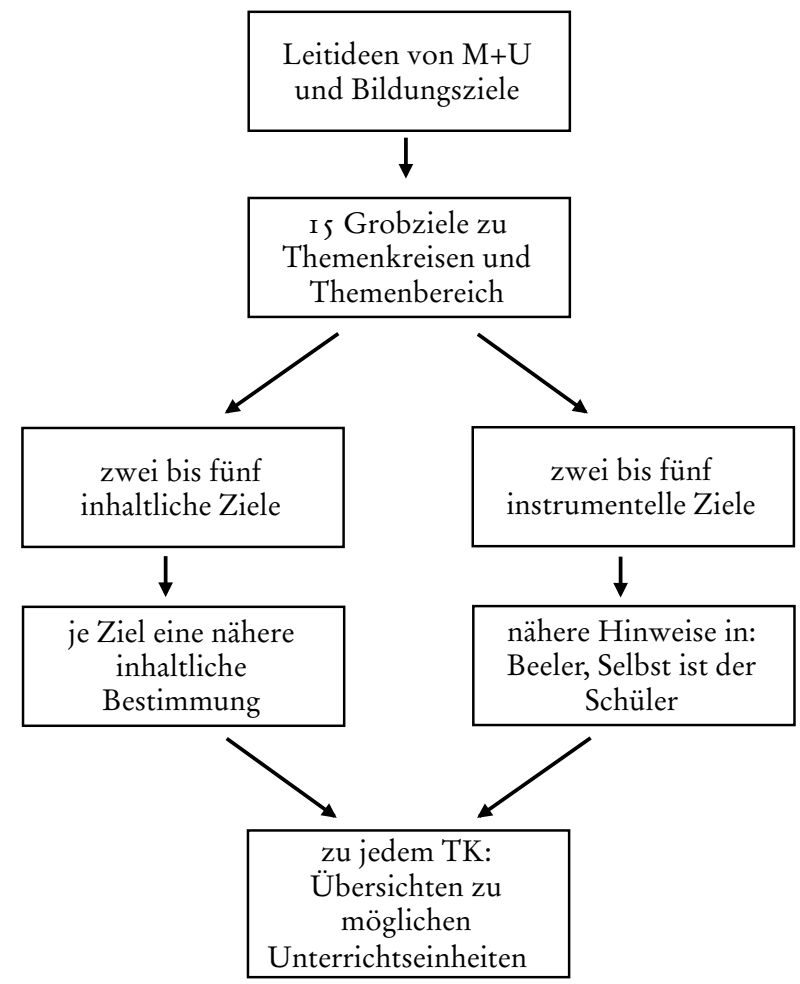

Quelle: Lehrplan «Mensch und Umwelt», November 1983, StALU, A 1253 9, S. 4

tion von Schulreformprojekten ab. Angesichts drängender schulstruktureller Herausforderungen auf der Volksschuloberstufe im Kanton Luzern setzte der Regierungsrat eine Kommission ein, deren Bericht die bildungspolitischen Bestrebungen aller IEDK-Kantone in den Folgejahren nachhaltig prägen sollte: Als Antwort auf den grossen Mangel an Lehrpersonen, die Problematik im Zusammenhang mit stark rückläufigen Schülerzahlen an bestimmten Standorten und angelehnt an die Forderung, die obligatorische Schulzeit auf neun Jahre zu verlängern, wurde eine organisatorische Zusammenführung benachbarter Schulstandorte $\mathrm{zu}$ sogenannten Schulzentren konzipiert. Angesichts politischer Forderungen nach erhöhter Durchlässigkeit im Schulsystem regte die Kommission zudem die Reorganisation der Volksschuloberstufe zur Orientierungsstufe an und lancierte insgesamt eine Debatte über die teilweise neu festzulegenden schulischen Inhalte und damit die Lehrpläne. 
Der interkantonalen Zusammensetzung der Kommission, der vergleichbaren Problemlage im Volksschulwesen aller Zentralschweizer Kantone und der Besetzung der Kommissionsleitung mit einem bestens vernetzten ehemaligen Mitglied der FAL ist geschuldet, dass der Kommissionsbericht eine interkantonale Lösungssuche auf der Ebene der IEDK angestossen, die Schaffung des Zentralschweizer Beratungsdienstes ermöglicht und Letzterem die Begleitung der anstehenden Reformen aufgetragen hat. Mit der Wahl von Anton Strittmatter als Nachfolger von Iwan Rickenbacher als Leiter des ZBS durch die IEDK wurde zudem begünstigt, dass ein curriculumbasiertes Verständnis von Schulreform in der Zentralschweiz Einzug halten konnte, wie es von der Freiburger Arbeitsgruppe zeit ihres Bestehens vertreten worden war.

Retrospektiv wenig erstaunlich, gelangte ein Kaderkurs unter der Leitung von Strittmatter nach einer fünftägigen Tagung 1978 zum Konsens, die anstehenden Lehrplanreformen für die Orientierungsschulen in der IEDK nach Grundsätzen zu realisieren, welche einstigen Strategiepapieren der FAL zum Verwechseln ähnlich sehen. ${ }^{109}$ In zweifacher Hinsicht kann die Realisierung jener Grundsätze beobachtet werden.

Der ZBS verfasste erstens Konzepte, nach welchen die einzelnen Phasen der Lehrplankonstruktion oder die Zusammensetzungen der Kommissionen für die Teilbereiche der Lehrpläne umgesetzt wurden. Vernehmlassungen zur breiten Abstützung aktueller Zwischenergebnisse in der Öffentlichkeit wurden ebenso zum Standard wie die aktive Mitarbeit von Lehrpersonen oder der Einbezug von wissenschaftlichen Experten in den Lehrplankommissionen.

Zweitens zeigt die Analyse von ab den späten i970er-Jahren entworfenen Lehrplänen, dass auch die inhaltliche Aufbereitung der schulischen Inhalte gemäss einstigen Modellen aus der Curriculumforschung erfolgte. Die Lehrplanarbeit in den Kommissionen war geprägt vom Formulieren von Leitideen, Grobzielen, dem Schreiben von Jahresstoffplänen und zusätzlichen Handreichungen für die Lehrpersonen.

I09 Einige dieser Strategien waren bereits in früheren Lehrplanreformprojekten im Kanton Freiburg unter Anleitung der FAL erprobt worden. Vgl. dazu das folgende Teilkapitel. 


\subsection{Das Freiburger EBAC-Projekt als «Erfahrungsfeld»}

Die Lernziele haben mit der Ausbreitung des Programmierten Unterrichts und der Curriculumforschung im besonderen sprunghaft an Bedeutung gewonnen. Zugleich ist aber auch die Problematik des Lernziels als Instrument in der Curriculumkonstruktion offensichtlicher geworden. Dennoch scheint sich die Funktion der Lernziele als Planungselement von Lernprozessen zunehmend auszuweiten. ${ }^{\mathrm{I} 10}$

Die Autoren des einleitenden Zitats übernahmen im Auftrag der Freiburger Erziehungsdirektion 1969 die Aufgabe, die bestehenden Lehrpläne für die deutschfreiburgischen Primarschulen dem neusten Stand der Forschung gemäss zu überarbeiten, wohl wissend, dass es jenen wissenschaftlichen Ansätzen teilweise an theoretischer Untermauerung und an Erfahrungen in der praktischen Umsetzung mangelte. Bis in die späten I970er-Jahre wurde unter breiter Beteiligung der Freiburger Lehrpersonen und unter wissenschaftlicher Anleitung und Beratung durch Mitglieder der Freiburger Arbeitsgruppe schrittweise der bisherige Lehrplan durch ein Curriculum ersetzt. Gleichzeitig wurde der gesamte Entwicklungsprozess aus curriculumtheoretischer Perspektive analysiert und beforscht.

Am Beispiel des Freiburger Lehrplanreformprojektes mit dem Namen «Entwicklung und begleitende Analysen eines Curriculums für die Primarschule» (EBAC) wird herausgearbeitet, inwiefern Erkenntnisse aus der Curriculumforschung in Bildungsreformprojekten rezipiert und teilweise implementiert wurden. Zusätzlich legt dieses Teilkapitel den Fokus stark auf die aus wissensgeschichtlicher Perspektive besondere Anlage des EBAC-Projektes: Die Hauptakteure der Schweizer Curriculumforschung konnten zu einem frühen Zeitpunkt ihrer Forschungstätigkeit erstmals in einem grösseren Projekt realisieren, was sie bisher an theoretischen Positionen in Fachzeitschriften zur Diskussion gestellt hatten. Damit einher geht die für dieses Kapitel leitende Frage nach etwaigen Rückwirkungen der Projektarbeiten auf die involvierten Konzepte aus der Forschung und allenfalls auf deren Weiterentwicklung. Dazu wird hauptsächlich auf den Quellenbestand zu Lehrplanreformen im Staatsarchiv des Kantons Freiburg zurückgegriffen. Ferner werden die Dokumentationen der FAL zum EBAC-Projekt berücksichtigt, welche im Zuge ihrer Begleitforschung verschiedentlich entstanden sind, sowie vereinzelt Archivalien der FAL beigezogen.

I IO K. Frey und U. P. Lattmann, EBAC-Bericht 4. Effekte der Operationalisierung von Lernzielen, 1970, S. 3, Archivalien der FAL. 
Im ersten Teilkapitel werden die Projektanfänge sowie die Projektziele und die Organisation erläutert. Darauf aufbauend stehen die Herausforderungen und Widerstände anlässlich der Bemühungen um eine breit abgestützte Lehrplanarbeit im Fokus. Der dritte Teil thematisiert das EBAC-Projekt als Praxisfeld für die Forschung.

\subsubsection{Beginn, Ziele und Organisation des EBAC-Projektes}

Ende Oktober 1969 wurde die «Freiburger Arbeitsgruppe für Lehrplanforschung» des Pädagogischen Institutes von der Erziehungsdirektion des Kantons Freiburg angefragt, an einer Lehrplanrevision für die Volksschulen (г. bis 8./9. Schuljahr) im deutschsprachigen Teil des Kantons Freiburg mitzuarbeiten. Da eine umfassende (globale) Lehrplan- oder Curriculumtheorie nur teilweise besteht oder in ihrer gegenwärtigen Form nicht ohne weiteres realisierbar ist, konnte sich die Mitarbeit der FAL nur auf Teilaspekte des Problemfeldes beziehen. ${ }^{\text {II }}$

Iwan Rickenbacher, selbst Mitglied der Freiburger Arbeitsgruppe, verfasste diese Zeilen 1970 für die «Schweizerische Lehrerzeitung». Das Zitat verdeutlicht, dass eine kantonale bildungspolitische Behörde auf die Wissenschaftler der Universität zugegangen war und sich damit derer Mitwirkung an der Reform versicherte. In diesem Zitat wird auch klar, dass zu Beginn der I970er-Jahre die Wissenschaftler mit einem theoretischen Handwerk zu Lehrplan- beziehungsweise Curriculumfragen zur Tat schritten, welches aus ihrer Sicht nicht ausgegoren war, und dass demnach etliche theoretische Teilaspekte noch ungeklärt bleiben mussten.

Bereits ab 1968 kursierte im pädagogischen Arbeitskreis der deutschsprachigen Schulen des Kantons Freiburg die Absicht, «die Ausarbeitung eines Führers und Lehrplanes für die deutschsprachigen Primarschulen» ins Auge zu fassen, zumal es dabei «um ein wichtiges Problem» gehe. ${ }^{112}$ Die Mitglieder des Arbeitskreises, zusammengesetzt aus dem Schulinspektorat, den Schuldirektoren, Vertretungen der Lehrpersonen und des Lehrerseminars ${ }^{113}$ sowie der Amtsleitung der deutschsprachigen Schulen in der Erziehungsdirektion, ${ }^{114}$ fassten im Rahmen

I I I Rickenbacher, 1970, S. 663.

I 2 Rechenschaftsbericht der Erziehungs- und Kultusdirektion des Kantons Freiburg für das Jahr i 968, S. 8, Forschungsbibliothek Pestalozzianum, FR HB I.

I 3 Das Lehrerseminar war im pädagogischen Arbeitskreis im Untersuchungszeitraum durch einen Methodiklehrer vertreten, das Lehrerinnenseminar hatte keine Vertretung.

I 4 Es handelte sich um die Erziehungs- und Kultusdirektion des Kantons Freiburg. Sie wird zugunsten der Lesbarkeit nachfolgend als Erziehungsdirektion bezeichnet. 
ihres regelmässigen Austausches in jenem Leitungsgremium den Entschluss, für diese Aufgabe zusätzlich die FAL vom pädagogischen Institut der Universität Freiburg anzufragen. Im Jahr darauf vermeldete der Rechenschaftsbericht des Kantons Freiburg: «Diese Gruppe ist mit der wissenschaftlichen Beratung der Arbeit beauftragt worden. Eine gewisse Anzahl von Primar-, Sekundar- und Mittelschullehrern sind zu Arbeitssitzungen einberufen worden, um Lernziele für die verschiedenen Unterrichtsfächer zu formulieren.» ${ }^{115}$

Aber worin genau bestand das angesprochene Problem, welches die Reform der Primarschullehrpläne initiiert hatte? Der einzige Hinweis fand sich in einem Projektbericht der FAL von 1972. Demnach war es bis in die späten I 960er-Jahre die Aufgabe der drei deutschfreiburgischen Schulinspektoren, für ihren Zuständigkeitsbereich jährlich eine Programmausarbeitung vorzunehmen. «Ein gewisser Koordinationsgedanke» sollte nun «eine Vereinheitlichung der Unterrichtsinhalte innerhalb des deutschsprachigen Kantonsteils herbeiführen». ${ }^{116}$ Es scheint, als ob die Vorgespräche zum geplanten Projekt zwischen Karl Frey von der FAL und dem zuständigen Amtsleiter von der Erziehungsdirektion es ermöglicht hätten, die angedachte Lehrplankoordination zu einer umfassenden Curriculumreform werden zu lassen.

Die Lehrplanarbeiten schritten zügig voran. Bereits im Frühjahr I 970 war die erste Erarbeitungsphase abgeschlossen. So verschickte die Erziehungsdirektion des Kantons Freiburg eine Konferenzeinladung für alle Lehrpersonen der deutschsprachigen Primarschulen des Kantons «zur Einführung in die Verwendung der Lernziele». ${ }^{117}$ Das dichte Tagungsprogramm war hauptsächlich bestimmt von Traktanden in der Verantwortung der FAL. Den Schulinspektoren oblag es, kurz in den Tag einzuführen sowie die Tagung abzuschliessen. Interessanterweise positionierte Urs Peter Lattmann von der FAL das Tagungsthema im ersten inhaltlichen Teil unter dem Titel «Der mögliche Beitrag des Lehrers für die Verbesserung des Unterrichts». ${ }^{118}$ Erst im zweiten Programmpunkt orientierte Kurt Aregger über Verlauf und Ziele des EBAC-Projektes, bevor Urs Isenegger dann die konkrete Einführung zu Bedeutung, Technik und Anwendung genau formulierter Lernziele im Unterricht übernahm. Die Lehrplanreform wurde den Lehrpersonen gegenüber als Instrument zur Unterrichtsoptimierung lanciert.

I I Rechenschaftsbericht der Erziehungs- und Kultusdirektion des Kantons Freiburg für das Jahr I969, S. 3, Forschungsbibliothek Pestalozzianum, FR HB I.

I 6 Aregger et al., 1972, S. I0.

I 17 Konferenzen für alle Lehrpersonen der deutschsprachigen Primarschulen des Kantons Freiburg, 4. 3. 1970, StAFR, DOA 2006/94.

i 8 FAL, I. Arbeitstagung für alle Primar- und Sekundarlehrpersonen, 4. 3. I970, StAFR, DOA 2006/94. 
Die Freiburger Lehrpersonen wussten bereits vor der Tagung, dass sich hinter dem Akronym EBAC der Projektname «Entwicklung und begleitende Analysen eines Curriculums» verbarg. Zusammen mit der Einladung wurde ihnen zur vorbereitenden Lektüre ein Arbeitsbericht der FAL über die ersten Monate dieses Lehrplanreformprojekts zugestellt. ${ }^{19}$ Sie fanden darin Informationen über die Kernpunkte der Anfänge des EBAC-Projektes und die weiteren geplanten Schritte. Das EBAC-Projekt wird im Folgenden kurz dargestellt.

Das EBAC-Projekt war darauf angelegt, «die Schulreform im Kanton Freiburg, vorerst auf der Primarstufe, wissenschaftlich zu begleiten», wobei zunächst «der Schwerpunkt auf die internen Strukturen des Unterrichts (Unterrichtsinhalte) gelegt» wurde. ${ }^{120}$ Dazu schrieb Iwan Rickenbacher präzisierend in der «Schweizerischen Lehrerzeitung», es gelte in der ersten Phase die für Lehrpersonen besonders bedeutsamen Bereiche der Lehrplanarbeit anzugehen: «Die Frage nämlich I. nach den Lernzielen («was müssen wir lehren`); 2. nach den Prüfverfahren (‘wie können wir feststellen, ob wir es gelernt haben`); 3. nach den Methoden (‘welches Material und welche Methoden eignen sich am besten, das zu lehren, was wir wollen $>$ ). ${ }^{121}$

Anders als in bisherigen Lehrplanreformprojekten hatten seit dem Projektbeginn im Jahr 1969 über 70 Lehrpersonen an der Findung und Formulierung von Lernzielen mitgearbeitet. Pro Fachbereich waren über I 200 Lernziele entstanden, welche auf über 200 Blättern in einem Ringordner abzulegen waren. ${ }^{122}$ Im Zuge dieses Prozesses sei mehr als ein traditioneller Lehrplan, aber weniger als ein Curriculum entstanden. Es müsse daher von einem Curriculumentwurf gesprochen werden, welcher, so die Sicht der Forscher der FAL, «laufend Änderungen und vor allem Ergänzungen [erfährt], die sich auf praktische und theoretische Erkenntnisse stützen». ${ }^{123}$

In der zweiten Phase ab Juni 1970 war anlässlich des EBAC-Projektes vonseiten der FAL geplant, die vorliegenden Lernziele nach ihrer Bedeutung zu gewichten und zu systematisieren, ihr Anspruchsniveau und damit die Angemessenheit für eine Verwendung auf der Zielstufe zu prüfen, laufend neue Lernziele zu entwerfen, ein Verfahren zur Überprüfung von Lernzielen zu erstellen sowie die Weiterbildung der Lehrpersonen weiterzuentwickeln. ${ }^{124}$ Rückblickend wurde diese zweite Phase, welche bis Mitte 1972 dauerte, als Phase der Revision bezeichnet. Ab Mitte 1973 zielte die dritte Phase des EBAC-Projektes dar-

I 9 EBAC-Projekt, Arbeitsbericht Nr. 2, I970, Archivalien der FAL.

I 20 Urs Isenegger, Jahresbericht der FAL zuhanden der Generalversammlung, 1972, Archivalien der FAL.

I 2 I Rickenbacher, 1970, S. 665.

I22 Aregger, I97Ia.

I 23 Ebd., S. 238.

I24 Rickenbacher, 1970. 


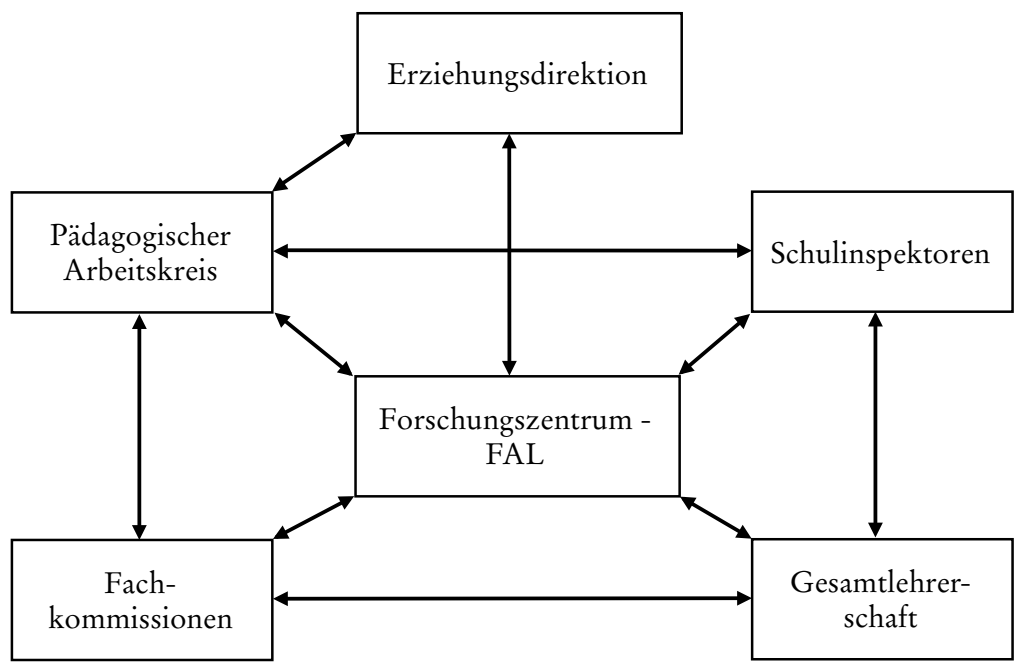

Quelle: Aregger et al., 1972, S. 4

auf ab, einerseits Unterrichtseinheiten für das Curriculum zu entwickeln und zu erproben, andererseits via Leitideen als didaktischen Bezugssystemen neue Curriculumteile zu entwickeln. ${ }^{125}$

Der Überblick über die Anfänge und die grobe Weiterentwicklung des EBAC-Projektes verdeutlicht erstens die Intention der FAL-Mitglieder, in der wissenschaftlichen Begleitung und Beratung der Lehrplanreform des Kantons Freiburg neue Ansätze aus der Curriculumforschung in den Reformprozess mit einfliessen zu lassen. Zweitens wird ersichtlich, dass das einst für die innerkantonale Lehrplankoordination angelegte Projekt zunehmend von den umfassenderen Reformintentionen der Curriculumforscher der FAL vereinnahmt wurde. Wie die folgenden Ausführungen zeigen, hat die Verteilung der Arbeitsbereiche auf die involvierten Akteure diese Entwicklung begünstigt.

Im ersten Tätigkeitsbericht der FAL über die Lehrplanreform an den deutschfreiburgischen Primarschulen ${ }^{126}$ liegt eine detaillierte Beschreibung der Aufgaben und Zuständigkeiten einzelner Akteursgruppen im Reformprozess vor. Passend dazu ist in der Publikation die Übersicht in Grafik I I abgebildet.

I 25 Brunner, Eigenmann und Mayer, 1977, S. 54.

I26 Aregger et al., I972. 
Die Aufgaben der einzelnen Akteursgruppen im EBAC-Projekt werden nachfolgend kurz umrissen, um ein klareres Bild der Projektorganisation zu ermöglichen:

- Die Erziehungsdirektion hatte im EBAC-Projekt die Aufgabe, den Auftrag für die Projektleitung beziehungsweise den Arbeitsvertrag zu formulieren, jede Projektphase auf Antrag zu genehmigen sowie die Beschlussfassungen über behördliche Erlasse vorzunehmen.

- Der pädagogische Arbeitskreis hatte die Aufgabe, die FAL zu unterstützen und die Erziehungsdirektion zu beraten.

- Die drei Schulinspektoren und der kantonale Schulinspektor hatten die Aufgabe, Fortbildungskonzepte zu beschliessen sowie die Erziehungsdirektion zu beraten und in den Fachkommissionen mitzuarbeiten.

- Die Fachkommissionen bestanden aus Lehrpersonen unterschiedlicher Schulstufen, Vertretungen aus der Fachwissenschaft, Schulinspektoren und Vertretungen aus der FAL. In den Fachkommissionen fand die praktische Curriculumentwicklung statt, dort wurden Begleituntersuchungen organisiert, die Anwendung neuer Curriculumelemente geprüft sowie die Durchführung von Fortbildungsveranstaltungen für die Gesamtlehrerschaft geplant.

- Die Gesamtlehrerschaft umfasste alle Lehrpersonen im Kanton Freiburg, welche mit dem Lehrplan arbeiten sollten. Ihnen wurde die Aufgabe zugedacht, Rückmeldungen bei praxisorientierten Problemstellungen zu geben und die FAL in der Projektgestaltung aus praktischer Perspektive zu beraten.

- Dem Forschungszentrum FAL wurde gemäss Projektbeschrieb die Aufgabe erteilt, die Projektplanung und -organisation zu übernehmen, die Erziehungsdirektion, den pädagogischen Arbeitskreis und Fachkommissionen wissenschaftlich zu beraten, Begleituntersuchungen zu planen und durchzuführen, theoretische Reflexionen über den Projektverlauf anzustellen, in der Planung und Durchführung der Lehrerfortbildung mitzuarbeiten und vieles mehr.

In aller Deutlichkeit zeigen die Ausführungen sowie die Grafik 9, dass die in diesem Projekt engagierten FAL-Mitglieder in einem intensiven Austausch mit allen weiteren Beteiligten standen. Sie waren in den Fachkommissionen und im pädagogischen Arbeitskreis vertreten, sie gestalteten die Weiterbildungsveranstaltungen für die Lehrpersonen, und sie formulierten die Projektausrichtung. Interessant ist die Tatsache, dass formell zwar eine Projektleitung existieren sollte, sie aber keiner Akteursgruppe zugeordnet war. ${ }^{127}$ Aufgrund der Präsenz der FAL in den Gremien und der damit verbundenen Planungs-, Organisations- 
und Beratungsmandate ist diese Aufgabe jedenfalls der FAL zuzuordnen. Mit Blick auf den Vertrag zwischen dem Erziehungsdepartement und der FAL wird allerdings auch klar, dass sich die FAL-Mitglieder in diesem Ausmass als wissenschaftliche Berater und Begleiter in die Reform einbringen mussten, zumal sie vom Konzept der wissenschaftlichen Beratung überzeugt waren, dass aus finanziellen Erwägungen bei Projektbeginn der Rückgriff auf externe Experten aber nicht vorgesehen war.

Im weiteren Verlauf des EBAC-Projektes hat sich die bestehende Projektorganisation als unzureichend herausgestellt, weshalb ein weiteres Gremium eingeführt wurde. Am ı०. Januar 1974 fand im Gebäude der Erziehungsdirektion die konstituierende Sitzung der «Planungsgruppe» (PG) statt, einer Gruppe mit dreifachem Auftrag: I. Beratung des pädagogischen Arbeitskreises, 2. Planung und Koordination der Lehrplanreform für die Primarschulen, 3. Kontaktpflege mit den anderen Schulstufen. ${ }^{128}$ Gemäss Protokoll lag die Ursache für die Gründung der Planungsgruppe im Folgenden: «Bisher war die FAL für die Reform verantwortlich. Sie plante und organisierte, die Lehrer waren die Ausführenden. Mit der PG soll die Reform demokratisiert werden.» ${ }^{129}$ Für den Vorsitz wünschte man sich eine Lehrperson, insbesondere unter Verweis «auf die Zurückhaltung und Skepsis vieler Lehrer gegenüber einer «Schulreform von oben>. Dies wäre der Fall, wenn die PG von einem Inspektor oder Methodiklehrer des Seminars geleitet würde.» ${ }^{130}$ Die Protokolle der weiteren Sitzungen zeigen allerdings, dass sich keine Lehrperson finden liess, welche der FAL die Verantwortung für die Lehrplanreform und die damit verbundene Fortbildung der Lehrpersonen abnehmen wollte. ${ }^{131}$

Zusammenfassend verdeutlichen die Anfänge der Lehrplanreformen im deutschsprachigen Teil des Kantons Freiburg, wie aus einem innerkantonalen Streben nach Harmonisierung der Lehrpläne aus der Feder der drei Schulinspektoren ein mehrjähriges Curriculumprojekt geworden war, welches bis zur Auflösung der FAL in den späten I970er-Jahren weiter bestehen sollte. Auf Initiative der kantonalen Bildungsbehörden wurde die FAL mit der Organisation und Planung der Reform beauftragt. Die Arbeitsgruppe der Universität Freiburg verknüpfte den Auftrag sogleich mit ihrem wissenschaftlichen Expertenwissen aus dem Bereich der Curriculumforschung. Ihre Mitglieder schufen mit Lehrpersonen besetzte Fachkommissionen und regten die Entwicklung

I 28 Protokoll der konstituierenden Sitzung der Planungsgruppe, Io. I. I974, StAFR, DOA 2006/I 89 .

I 29 Ebd., S. 2.

I30 Protokoll der konstituierenden Sitzung der Planungsgruppe, I0. I. 1974, S. 3 f., StAFR, DOA 2006/I 89 .

I3 I Vgl. Protokolle der Planungsgruppe, I6. I., I. 7., 2. 9., I0. 3. I975, StAFR, DOA 2006/I 89. 
von überprüfbaren Lernzielen an. Innert relativ kurzer Zeit entstanden Hunderte Lernziele, welche in noch ungeordneter Form provisorisch als neuer Lehrplan eingeführt wurden, nicht ohne alle Lehrpersonen in Weiterbildungen damit vertraut zu machen. Gleichzeitig machte die Gruppe um Karl Frey das Lehrplanprojekt zu ihrem Forschungsgegenstand. Sie plante Weiterentwicklungen der Lehrplanarbeit aufgrund ihrer begleitenden Analysen. Zudem waren die FAL-Mitglieder bestrebt, die ihnen zugedachte Leitungsfunktion des EBAC-Projektes abzugeben und den Lehrpersonen anzuvertrauen, ganz im Sinne einer Demokratisierung der Lehrplanarbeit. Dass dieser Prozess auch einige Schwierigkeiten mit sich brachte, zeigen die folgenden Analysen. ${ }^{32}$

\subsection{2 "Demokratische Lehrplanarbeit» - Herausforderungen und Widerstände}

Man will heute alles auf eine breite Basis stellen, man will möglichst alles mitreden und mitentscheiden lassen. Dieses neue Konzept bringt bestimmt nicht überall gute Früchte hervor. ${ }^{133}$

Kaum waren die ersten Monate des EBAC-Projektes verstrichen, kritisierte ein sichtlich skeptischer Schulinspektor gegenüber dem Amtsvorsteher der Freiburger Erziehungsdirektion den Beschluss, die Lehrpersonen mit in die Entwicklung von Lehrplanelementen einzubeziehen. Der Ärger wäre ihm ja nicht zu verübeln gewesen, immerhin war erst kürzlich der Schwerpunkt der Lehrplanarbeit von den Schulinspektoren weg zu den Lehrpersonen verlagert und unter die Anleitung und Beratung der FAL gestellt worden. In diesem Brief hingegen zielte die Kritik des Inspektors auf die vermeintlich mangelnde Distanz und Abstraktionsfähigkeit der Lehrpersonen: «Bezüglich Lehrplan geht meine Ansicht immer noch etwa dahin, dass viele Lehrer und Lehrerinnen in der Schulstube sehr gut arbeiten können, für die Erarbeitung eines Lehrplanes aber oft zu befangen sind in ihrer tagtäglichen Arbeit und so die ganze Sache zu wenig von oben sehen. Man kann mir entgegnen, dass die Herren des Päd. Instituts das Gegengewicht bilden. Das kann ich nicht bestreiten.» ${ }^{134}$

I32 Vorab ist zu bemerken, dass sich das folgende Stimmungsbild bezüglich Herausforderungen und geäusserter Kritik an Curriculumreformen durchaus vergleichbar an einigen deutschsprachigen Kantonen herausarbeiten liesse, in dieser Arbeit exemplarisch präsentiert am Beispiel des EBAC-Projektes.

I33 Ein Schulinspektor, 20. 7. 1970, StAFR, DOA 2006/19.

I34 Ebd. 
Die Schwierigkeiten in der von der FAL konzipierten Reform der Lehrplanarbeit schienen zusätzlich in der Pluralisierung der in den Reformprozess involvierten Akteure verortet. Bis anhin oblagen Ausarbeitung und Feinschliff der Lehrpläne «einer kleinen Gruppe von Lehrern, Schulinspektoren oder Schulräten». ${ }^{135}$ Die oben beschriebene Organisation mit Kommissionen, dem pädagogischen Arbeitskreis, dem Schulinspektorat und der FAL verursache Unklarheiten. Vor diesem Hintergrund bestellte Anfang Dezember 1970 der Amtsleiter aus der Erziehungsdirektion die Schulinspektoren zu einer Besprechung mit dem «Gesprächspunkt: Sinn und genaue Gestaltung der Tagungen». ${ }^{136}$ Anlass für die Unterredung war eine seit einiger Zeit bestehende Spannung hinsichtlich der Rollenverteilung in der Reform generell und an den Tagungen für Lehrpersonen zur Einführung in den neuen Lehrplan im Speziellen. Bereits im März 1970 richtete sich der Abteilungsleiter brieflich an Kurt Aregger von der FAL und hielt fest, «dass die Konferenzen jeweils unter der Verantwortung der betreffenden Herren Inspektoren stehen und von diesen eröffnet werden» ${ }^{137}$ Gleichentags schrieb er an die Schulinspektoren: «Von verschiedenen Stimmen aus dem Publikum habe ich vernommen, dass viele Lehrer den Eindruck haben, die FAL arbeite auf eigene Mühlen und betrachte die Lehrer als «quantité négligeable. Aus diesem Grund möchte ich Sie bitten, an den Konferenzen «das Heft in den Fingern zu behalten, oder eventuell Lehrer mit dieser moralischen Aufgabe zu beauftragen.» ${ }^{138}$

Die beschriebenen Ungereimtheiten liessen sich mit diesen Interventionen aus der Erziehungsdirektion nicht beiseiteschaffen. Sie gaben weiterhin Anlass zu schriftlichen Stellungnahmen und Aussprachen zwischen den Beteiligten. ${ }^{139}$

Demgegenüber machten den Lehrpersonen weniger die von der FAL in den Prozess eingebrachten Grundsätze der Partizipation oder Demokratisierung von Lehrplanarbeit zu schaffen als die damit einhergehende Mehrbelastung. Für das Engagement der Lehrpersonen in Projekt- oder Arbeitsgruppen waren keinerlei Entlastungen oder finanzielle Abgeltungen vorgesehen. ${ }^{140}$

Die Freiburger Erziehungsdirektion hatte zur Findung von Lehrpersonen für die Lehrplankommissionen eigens ein standardisiertes Formular erstellt und damit breitflächig zur Mitarbeit aufgerufen. Kaum war das Projekt aber ange-

I35 Rickenbacher, 1970, S. 665 .

I36 Abteilungschef der Erziehungsdirektion, 3. I2. 1970, StAFR, DOA 2006/94.

I37 Abteilungschef der Erziehungsdirektion, 4. 3. 1970, StAFR, DOA 2006/94.

I 38 Ebd.

I39 Vgl. beispielsweise Dokumente vom I4. 6., 29. 9. I972, StAFR, DOA 2006/94.

I40 Im Schuljahr 1972/73 musste das EBAC-Projekt ganz auf die Mitarbeit der Lehrpersonen verzichten. Die Lehrpersonen des Kantons legten sämtliche freiwilligen beziehungsweise unentgeltlichen Arbeiten nieder; sie standen mitten in festgefahrenen Lohnverhandlungen mit der Erziehungsdirektion. Vgl. Brunner et al., I977. 
laufen, erreichten die Erziehungsdirektion immer wieder Abmeldungen. Die Lehrpersonen machten meistens schulische und private Gründe geltend: «Aus nachfolgenden Gründen kann ich leider an der Lehrplanreform 1971/72 nicht mehr mitmachen: I. Arbeitstempo, 2. Übernahme einer Klasse v. 40 Schülern, 3. Schulhausneubau in Düdingen.» ${ }^{141}$ Bisweilen betonten die zur Mitarbeit Aufgerufenen aber auch, dass sie sich für diese Aufgabe schlicht nicht qualifiziert genug fühlten. ${ }^{\mathrm{I} 2}$

Nebst der zusätzlichen zeitlichen Belastung fürchteten die Lehrpersonen den Verlust von Gestaltungsfreiheit im Zuge der Neuerungen aus der Curriculumforschung, wie sie mit dem EBAC-Projekt in den Schulen Deutschfreiburgs angekommen waren. Lehrpersonen taten ihr Unverständnis öffentlichkeitswirksam in Lehrerzeitschriften kund, wie die folgenden Einblicke zeigen.

1973 war ein ungewöhnlich langer Artikel in der «Schweizerischen Lehrerzeitung» wie folgt übertitelt: «Curriculumforschung - Weg in Entmenschlichung und Bildungsdiktatur? $\gg^{143}$ Es handelte sich dabei um die flammende Rechtfertigung von Curriculumreformen im Schulwesen, welche durch eine Serie von Leserbriefen ausgelöst worden war. So störte sich ein Kritiker an den Wogen von Wortneuschöpfungen und Begriffskomposita: «Lernzielorientierter Unterrichtsvorbereitungs-Ordner», «Eingangsvoraussetzungen», «Verhaltensänderungsprozesse». ${ }^{144}$ Die Sprache der Curriculumforschenden sorgte im wahrsten Sinne des Wortes für Unverständnis, sodass ein Leserbrief monierte: «Und am Ende dann [...] - ist nicht einmal klar geworden, was das sein soll: ein Curriculum. Wunderliche Wissenschaft. ${ }^{\text {I4 }}$

In einer anderen Ausgabe der «Schweizerischen Lehrerzeitung» wurde ein fiktives Interview abgedruckt, durchgeführt in der fernen Zukunft mit dem Leiter der «SUMACUCO», was so viel bedeuten sollte wie «Schweizerische Unterrichtsmitschauanlage für Curriculumkontrolle». ${ }^{146}$ Von dieser hoch technifizierten Anlage mit dem Charme einer Raumfahrtzentrale aus könne man, so wird erklärt, jederzeit jede beliebige Lehrperson überwachen. Sollten dabei Unregelmässigkeiten wie das unerlaubte Ausschalten des Sprachlabors festgestellt werden, werden sie, die neu überdies «Unterrichtsbeamten» genannt werden, zur Korrektur in Bewährungskurse für Rückfällige gesteckt, und zwar in sogenannten Technological Centers. Damit werde der folgende entscheidende Fortschritt erzielt: «Wir haben nun endlich die Unterrichtsbeamten [...] eine

I4I Eine Lehrperson. Arbeit am Lehrplan, 20. 8. I97I, StAFR, DOA 2006/i 89.

I42 Eine Lehrperson. Weiterführung der Lehrplanarbeiten, 7. I0. 1970, StAFR, DOA 2006/189.

I43 Strittmatter, I973.

I 44 Muntwyler, I973, S. 209.

I45 Ebd.

I46 S. n., I972, S. 59I. 
der eigenwilligsten Gesellschaftsgruppen, die zu integrieren äusserst schwierig war, die haben wir nun im Griff. Alle früheren Versuche, ihre Leistung zu kontrollieren, sind bekanntlich gescheitert. Denn auch die Kontrolleure, man nannte sie je nach Gegend Inspektoren, Visitatoren, Schulpfleger und ähnlich, waren nicht frei von allzu menschlichen, ja sogar menschlichen Regungen, die heute erziehungswissenschaftlich als Irritationen bezeichnet werden.» Diese schalkhafte Parodie auf Diskussionen rund um Curriculumreformen zeichnete ein orwellsches Szenario von Überwachung, Kontrolle und verkümmerter Gestaltungsfreiheit der Lehrpersonen, welches mit der Einführung von Lernzielen im Unterricht begründet wurde. Weil Lernziele wie im EBACProjekt als Feinziele für den Unterricht im Lehrplan fixiert und vorgegeben wurden, fürchteten Lehrpersonen um ihre Methodenfreiheit. Darüber hinaus wurde die Technisierung durch Sprachlabore und generell eine Planungsgläubigkeit kritisiert, die konträr zu pädagogischen Grundüberzeugungen einiger Lehrpersonen zu stehen schienen. Das Formulieren von Lernzielen möge für kognitive Lernbereiche teilweise umsetzbar sein, für das Gemüt des Kindes sei aber kein Platz, so die Kritik.

Insgesamt weisen die Analysen auf drei Konfliktbereiche hin, welche sich in den ersten Umsetzungen der Freiburger Curriculumentwicklung abgezeichnet haben. Erstens haben die Versuche, die Lehrplanarbeit zu demokratisieren, vereinzelt zu Zerwürfnissen zwischen der Freiburger Arbeitsgruppe und dem Schulinspektorat geführt. Die Aufgabenbereiche in den Entwicklungsprozessen selbst sowie in der Weiterbildung und Kommunikation mit den Lehrpersonen waren offenbar nicht eindeutig genug abgesteckt, wenngleich die Schulbehörden und Lehrpersonen als «Träger» der Entwicklungsarbeiten vorgesehen und der FAL Planung und Organisation zugeschlagen worden waren. Zweitens wurde bei der Gründung von Kommissionen zur Entwicklung von Lernzielen vorausgesetzt, dass die Lehrpersonen bereit wären, diese Aufgabe zusätzlich zu den bestehenden Verpflichtungen und unentgeltlich zu übernehmen. Nach etlichen Schwierigkeiten, Lehrpersonen für die Kommissionen zu gewinnen und dort auch zu halten, kulminierte die Kooperationsverweigerung der Lehrpersonen gegenüber der Erziehungsdirektion 1972/73. Sie legten sämtliche freiwillig geleisteten Arbeiten nieder, um in Lohnverhandlungen ihren Positionen Nachdruck zu verleihen, und brachten damit das EBAC-Projekt für ein Jahr zum Stillstand. Schliesslich meldeten sich einige Lehrpersonen auch in Lehrerzeitschriften zu Wort und verliehen ihren Vorbehalten und Ängsten Ausdruck, wonach durch die Einführung lernzielorientierter Lehrpläne ihr Unterricht kontrollierbar, fremdgesteuert, einförmig und seelenlos werden würde. 
4.3.3 Das EBAC-Projekt als «Erfahrungsfeld» für die Forschung

Die EBAC-Projekte stellen die Erfahrungsfelder für die mehr auf Theoriebildung, auf übergreifendere, allgemeinere Fragen ausgerichteten Forschungsziele des Nationalfonds-Projektes dar. ${ }^{\mathrm{I} 47}$

Eine Eigenheit des EBAC-Projektes in Freiburg bestand darin, dass es von der Erziehungsdirektion vergleichsweise früh als Curriculumentwicklungsprojekt initiiert wurde, obwohl die theoretischen Grundlagen für die angestrebten Zielsetzungen nicht vollständig erarbeitet waren. Dass die schulischen Inhalte in den Lehrplänen neu zu einer Ansammlung von Lernzielen in einem Curriculum umgearbeitet würden, war als Stossrichtung wohl vereinbart, nur war unklar, wie etwa diese Entwicklung unter Mitarbeit der Lehrpersonen theoretisch wie organisatorisch angegangen werden sollte. In einem Bericht der FAL von 1970 ist dazu zu lesen: «Die vorhandenen Operationalisierungstechniken, insbesondere die bekannteste von Mager (1969), sind unzureichend (vgl. Lange 1967). Gleichwohl wird empfohlen, Lernziele zu operationalisieren.» ${ }^{148}$

Die involvierten Wissenschaftler der FAL berichten rückblickend, sie hätten zwar von Beginn weg lernzielorientierte Lehrpläne entwickeln wollen, wie das am besten gelingen könne, habe man im Laufe des Projektes aber zunächst herausfinden müssen. ${ }^{149}$ Die gross angelegten Weiterbildungsveranstaltungen für Lehrpersonen waren mithin Entstehungsanlass für Konzeptpapiere und Anleitungen dazu, wie Lernziele im Verbund zu formulieren seien. ${ }^{150}$ Nicht am Schreibtisch, sondern beispielsweise in Projektsitzungen anlässlich gesammelter Erfahrungen aus dem Reformprozess entstanden Überlegungen zur Koordination und Kooperation zwischen allen in den Prozess mit einzubeziehenden Akteuren. ${ }^{\text {s } 1}$ Selbst die Frage, inwiefern die Zusammensetzung der Lehrplankommissionen eine Auswirkung auf die Resultate der Lernzielfindung habe, blieb nicht unerforscht. ${ }^{152}$

Ganz in sozialwissenschaftlicher Manier ermittelte die FAL mittels Fragebogenerhebungen die Verwendung und Verwendbarkeit der entstandenen Curriculumentwürfe bei Lehrpersonen. Im Begleitbrief zur zweiten Befragung im Juli 1971 wurde begründet: «Dieser 2. Fragebogen soll aber nicht nur dazu

I47 Forschungszentrum FAL, Tätigkeitsbericht 1974, Februar 1975, S. I0, Archivalien der FAL.

I48 K. Frey und U. P. Lattmann, EBAC-Bericht 4. Effekte der Operationalisierung von Lernzielen, 1970, S. 3, Archivalien der FAL.

I49 Isenegger, 2016; Lattmann, 2015 ; Rickenbacher, 1970.

I 50 Eigenmann und Strittmatter, 1972.

I 5 I Strittmatter, I978a.

I 52 Rickenbacher, 1970. 
dienen, interessante Vergleichsstudien zu betreiben, sondern er ist in erster Linie als Instrument eingesetzt, um fortwährende Verbesserungen des Curriculums vornehmen zu können.» ${ }^{153}$ So wurde gefragt nach der Häufigkeit der Verwendung des Curriculumentwurfes, in welchen Fächern dieser verwendet werde, wozu der Curriculumentwurf überhaupt genutzt werde, aber auch wie die Lehrpersonen die Weiterbildungsveranstaltungen erlebt hatten.

Schliesslich war die praktische, projektgebundene Entwicklungsarbeit im Rahmen des EBAC-Projektes ebenso Impulslieferant für die Theoriearbeiten der Wissenschaftler, welche dann wiederum in nationalfondsgeförderten Projekten untersucht worden sind. ${ }^{54}$ Aus der projektbegleitenden Forschung sind so vier Dissertationen entstanden, die von Joseph Eigenmann, Bruno Santini, Kurt Aregger und Joe Brunner. ${ }^{\text {Iss }}$ Aber auch vom EBAC-Projekt losgelöste Promotionsarbeiten der FAL-Mitglieder haben an Problemstellungen aus dem Projekt angeknüpft. Kurt Bossart etwa publizierte zu Verfahren der Lernzielerhebung. ${ }^{156}$ Er thematisierte unter anderem eines der Grundprobleme aus den Anfängen des EBAC-Projektes: die Einteilung und Typisierung von Lernzielen. Der Vorschlag, die Lernziele nach einem übergeordneten «Begründungszusammenhang» ${ }^{157} \mathrm{zu}$ ordnen oder umgekehrt von einem solchen herzuleiten, kursierte also in Publikationen der FAL unter dem Begriff Leitidee. Leitideen gewannen zunehmend an Popularität und wirkten auf das EBAC-Projekt zurück: «Im Projektjahr 1974/1975 wurde intensiv an der Entwicklung von Leitideen für die Primarschulen gearbeitet», ${ }^{158}$ bevor sie später als Instrument zur Vergewisserung über die Ziele von Schule in Lehrplänen weiterer Kantone verwendet wurden. ${ }^{59}$

Insgesamt stehen die Ausführungen in diesem Teilkapitel für die Anfänge der Begleitforschung im Bereich der Curriculumentwicklung und zeigen, dass die Mitarbeiter der FAL von Beginn weg nicht nur gemeinsam mit Lehrpersonen Lernziele für den deutschfreiburgischen Primarschulunterricht entwickelten, sondern den gesamten Prozess aus ihrer Forschungsperspektive begleiteten und dazu publizierten. Was die zur Beratung, Begleitung und Organisation des EBAC-Projektes beauftragten Mitglieder der FAL in der praktischen Arbeit an Problemstellungen beobachten konnten, hat sie zur weiteren Beschäftigung angeregt. Sie verfassten einerseits Artikel und Buchbeiträge, um über ihre Erfahrungen und Feststellungen im Projekt zu berichten. Andererseits setzten sie

I53 Begleitbrief zum 2. Fragebogen, Juli 197I, StAFR, DOA 2006/94.

I54 Vgl. dazu Kapitel 2.3.

I55 Aregger, 1974b; Brunner, 1980; Eigenmann, I975; Santini, I971. Vgl. auch Kapitel 2.3.

I56 Bossart, 1975b.

I 57 Isenegger, 1972, S. 3 I.

I 8 Tätigkeitsbericht Forschungszentrum FAL von 1975, Archivalien der FAL, S. 5.

I 59 Mayer, I984; F. Oggenfuss, 1984. 
sich theoretisch mit vorgefundenen Problemstellungen auseinander und suchten nach Antworten, welche teilweise im weiteren Projektverlauf wieder als Verfahren zur Problembearbeitung rezipiert wurden.

\subsubsection{Curriculum(theorie)entwicklungsprojekt in Freiburg}

Ich glaube, dass dieser Lehrplan letztendlich etwas Brauchbares und Nützliches werde. Aber bis er das sein wird, mag es mit all den durchzuspielenden Experimenten 1972 oder gar 1973 werden. Man hat sehr weit ausgeholt und viel Wissenschaftlichkeit herbeigeholt. ${ }^{160}$

Mit dem Weggang der FAL von der Universität Freiburg ${ }^{161}$ wurde auch das EBAC-Projekt im Kanton Freiburg nach knapp zehn Jahren eingestellt. Seinem Namen gemäss war das Projekt von Anfang an darauf angelegt, einerseits ein Curriculum für die Freiburger Primarschulen zu entwickeln, andererseits diesen Prozess begleitend zu analysieren. Per Beschluss der Freiburger Erziehungsdirektion von 1969, die Lehrpläne für die deutschfreiburgischen Schulen einander anzugleichen und zu erneuern, wurde die FAL mit der Projektorganisation und Koordination betraut. Sie war zudem aufgefordert, die wissenschaftliche Beratung und die begleitenden Analysen zu übernehmen.

Wie dem einleitenden Zitat zu entnehmen ist, dauerten die Entwicklungsarbeiten länger als zunächst projektiert. Zwar konnte bereits nach einem Jahr ein erster Entwurf für ein neues, lernzielbasiertes Primarschulcurriculum vorgelegt werden. Aus mindestens drei Gründen mündeten diese Arbeiten aber in ein noch Jahre dauerndes Entwicklungsprojekt. Erstens wurden die Lehrpersonen, die Schulbehörden und alle weiteren Interessierten in die Entwicklung mit einbezogen. Sie waren auf Weiterbildungen angewiesen und hatten sich auf eine nicht unumstrittene Projektorganisation einzulassen. Zweitens vermittelten die Mitglieder der FAL als wissenschaftliche Autoritäten in diesem Prozess die Vorstellung eines Curriculums als Instrument für eine «permanente Schulreform». ${ }^{162}$ Demgemäss war jedes Arbeitsergebnis immer nur Zwischenprodukt, bis es wieder überarbeitet würde. Drittens war selbst den Mitgliedern der FAL nur klar, in welche Richtung die Entwicklung eines Curriculums gemäss ihren wissenschaftlich abgestützten Vorstellungen gehen könnte. Handlungsanweisungen zur Formulierung von Lernzielen mussten aber auch sie im Projekt-

I60 Ein Schulinspektor brieflich an den Abteilungschef Erziehungsdirektion Freiburg, 19. 9. 1970, StAFR, DOA2006/I 89 .

I6I Vgl. dazu Kapitel 2.4.

I62 Brunner et al., I977, S. 55 . 
verlauf erst entwickeln. Der Stand der Forschung in diesem Bereich war unzureichend.

Die Analysen zur Begleitforschung der FAL wie auch zu den Herausforderungen und Widerständen im Zuge der Projektumsetzung haben dargelegt, dass auf Geheiss der Freiburger Erziehungsdirektion von der FAL entwickelte Modelle und Ansätze aus der Curriculumforschung in konkrete Entwicklungsarbeiten im Kanton übersetzt wurden: Lehrpersonen mussten Weiterbildungsveranstaltungen absolvieren, Lernziele formulieren, Leitideen ausarbeiten. Wegen einiger erst in der konkreten Projektarbeit absehbarer Problemstellungen erwies sich das EBAC-Projekt zudem als Anlass für die Weiterentwicklung curriculumtheoretischer Modelle, welche dann in Publikationen mündeten und letztlich wiederum in der Praxis rezipiert wurden.

\subsection{SIPRI - ein «Animationsprojekt» der EDK für die Primarschule}

Ein Animationsprojekt der Pädagogischen Kommission der EDK. SIPRI will zeigen, wo die Primarschule heute steht, damit Lehrer, Wissenschafter und Verwaltung gemeinsam konkrete Verbesserungen vorschlagen und erproben können. ${ }^{163}$

Die zweite Informationsbroschüre für alle Interessierten, insbesondere aber für die Lehrpersonen der deutschsprachigen Kantone, versuchte mit diesen ersten Sätzen den Leserinnen und Lesern das Ziel des interkantonalen «Animationsprojektes» zu vermitteln: die «Verbesserung» der Primarschulen.

Hinter dem Namen SIPRI verbarg sich ein Projekt, welches dem Namen nach etwas bescheidener zur «Überprüfung der Situation der Primarschulen» anleitete. Von 1978 bis 1986 realisierte die Pädagogische Kommission der EDK auf Initiative der Konferenz der Schweizerischen Lehrerorganisationen (KOSLO) ein Gesamtprojekt, welches in Anbetracht des Umfangs ihrer Absichten und Zielsetzungen in vier Teilprojekte aufgeteilt wurde. Das erste Teilprojekt führte Lehrpersonen, Personal aus den kantonalen Bildungsverwaltungen wie auch Forschende im Bereich der Erziehungswissenschaft zusammen. Sie alle beschäftigten sich während der achtjährigen Projektdauer mit Lehrplänen, ihrer Bedeutung für den täglichen Schulunterricht und der Angemessenheit der darin formulierten Erwartungen an das Lernen der Schülerinnen und Schüler.

Wie die bisherigen Teilkapitel zu den Schulreformprojekten im Kanton BaselStadt, in der Zentralschweiz und im Kanton Freiburg analysiert dieses vierte 
Teilkapitel ein Projekt, im Zuge dessen die Lehrpläne als Instrument der Reform begriffen wurden und in den Fokus der Veränderungsintentionen rückten. Wiederum stehen die Fragen im Zentrum, welche Probleme überhaupt eine Reform schulischer Lehrpläne anstiessen, wie Lehrpläne verändert werden sollten und wozu sowie besonders auf welche Wissensbestände in welcher Form zurückgegriffen wurde während der Reformbemühungen.

Zunächst werden die Beweggründe und kontextuellen Ursachen erläutert, welche zur Realisierung eines Grossprojektes im Auftrag der EDK führten. Anschliessend fokussiert das Kapitel auf dasjenige der vier Teilprojekte von SIPRI, welches die Lehrpläne der Primarschule ins Zentrum der Projekttätigkeiten stellte. In drei Schritten werden dann die Projekttätigkeiten analysiert.

\subsubsection{Die Situation der Primarschulen ist zu überprüfen}

Am 26. Oktober 1978 beschloss die EDK die Durchführung ihres ersten Grossprojektes. In Zusammenarbeit zwischen der pädagogischen Kommission der EDK und der Konferenz der Schweizerischen Lehrerorganisationen (KOSLO) wurde das Projekt seit 1976 ausgearbeitet, welches auf die Überprüfung der aktuellen Situation der Primarschulen hin angelegt war. ${ }^{164}$ Stein des Anstosses war eine an die Kantone gerichtete Empfehlung der EDK von 1975, wonach der Unterricht in der zweiten Landessprache auf die Primarschulen vorzuverlegen war. Infolgedessen befürchteten die Primarlehrpersonen eine Überlastung ihrer Schülerinnen und Schüler und ihrer selbst und forderten eine grundsätzliche Überprüfung der Situation der Primarschulen. Demgemäss wurden die folgenden Projektziele entworfen und in einer Sondernummer der «Schweizerischen Lehrerzeitung» breit publiziert: ${ }^{165}$

- wesentliche Aspekte des Ist-Zustandes der Primarschule erfassen

- Vorschläge für konkrete Verbesserungen und für neue Lösungen von Aufgaben in der Primarschule formulieren und nach Möglichkeit erproben

- umfassendere Grundlagen für die Weiterentwicklung der Primarschule bereitstellen und die Zusammenarbeit zwischen den Kantonen auf diesem Gebiet fördern

- in den interessierten Kreisen (Lehrerschaft, Schulverwaltung, Öffentlichkeit, Forschung) eine intensivere Auseinandersetzung mit Fragen und Problemen der Primarschule auslösen und ein Überdenken der Aufgabe dieser Schulstufe fördern sowie auf neue Möglichkeiten zu ihrer Erfüllung hinweisen 
Bis anhin, so wurde von bildungspolitischer Seite argumentiert, sei die Primarschule von den «stürmischen pädagogischen Entwicklungen» im schweizerischen Schulwesen seit den i 960er-Jahren «verschont» geblieben. ${ }^{166}$ Gleichwohl seien stets neue Anliegen wie etwa solche nach mehr Medienpädagogik, Sexualoder Verkehrserziehung an sie herangetragen worden, ohne je «die Überlastung bzw. die Kopflastigkeit der Lehrpläne als Hauptübel» anzuerkennen. ${ }^{167}$ Auch die Resultate einer Befragung von Primarschullehrpersonen deuteten auf diesen Missstand hin. Vor dem Hintergrund wurde anlässlich des SIPRI-Projektes die Primarschule mit ihren Aufgaben und Herausforderungen unter die Lupe genommen, wobei es «weder um das «Modernisieren noch um eine Kehrtwendung [geht], sondern um ein Abwägen von dem, was die Primarschule leisten soll, welche Erziehungsfunktionen erfüllt werden können, welche Lerninhalte beibehalten, ergänzt, erneuert oder weggelassen werden sollten, wie die Unterrichtsorganisation und -methoden verbessert werden können». ${ }^{168}$

Die Vielfalt der oben formulierten Ziele des Projektes sowie die Breite der hier aufgezählten und zu überprüfenden Bereiche deuten bereits darauf hin, dass der Dringlichkeit der gegebenen Herausforderungen entsprechende Schwerpunkte herausgearbeitet wurden. Unter Einbezug der EDK-Regionalkonferenzen und der KOSLO lagen letztlich vier Teilprojekte vor: I. die Lernziele der Primarschule: Anspruch und Wirklichkeit, 2. die Funktionen und Formen der Schülerbeurteilung, 3. der Übergang von der Vorschulperiode zur Primarschule, 4. Kontakte und Zusammenarbeit zwischen Schule und Elternhaus.

Die folgenden Ausführungen fokussieren auf die Analyse des ersten Teilprojektes. ${ }^{69}$ Wie sich zeigen wird, wurden Lehrpläne im Rahmen der Projektarbeiten als die zentralen Dokumente, als Regelwerk für die Primarschulen interpretiert, die Nutzung der Lehrpläne durch die Lehrpersonen und damit deren Bedeutung für den Unterricht thematisiert sowie davon ausgehend die Belastung der Schülerinnen und Schüler der Primarschule thematisiert. Zunächst bedarf es noch einer Vorbemerkung zur Projektorganisation, welche zwar vornehmlich die anderen Teilprojekte prägte, insgesamt aber eine Eigenheit von SIPRI ausmachte.

In das Gesamtprojekt zur Überprüfung der Situation der Primarschulen sollten «möglichst alle Partner des Erziehungswesens einbezogen» und «gewisse fest-

I66 J. P. Meylan, 1979, S. 626; Trier, 1979.

I67 Salamin, I979, S. I 577.

I68 J. P. Meylan, I979, S. 627.

I69 Die Auseinandersetzung mit Lehrplänen wurde vorrangig im Teilprojekt I geleistet. Allerdings wurden auch in den drei weiteren Teilprojekten Diskussionen geführt und Arbeitspapiere erstellt, deren Vorschläge die Lehrpläne betrafen. Die gesammelten Resultate wurden in einem Dossier festgehalten; Vgl. Schweizerische Konferenz der kantonalen Erziehungsdirektoren, 1985 . 
gefahrene Gewohnheiten, so zum Beispiel die typische Arbeitsform der reinen Expertenkommission, überwunden werden». ${ }^{170}$ Dieser Ansatz veranlasste die Organisatoren von SIPRI dazu, alle Kommissionen und Leitungspositionen möglichst ausgewogen mit Lehrpersonen, Personal aus den Schulverwaltungen und Forschenden aus der Erziehungswissenschaft zu besetzen. Zusätzlich wurden sogenannte Projektschulen verschiedentlich in die Teilprojekte mit einbezogen. Im Grundsatz zielte das Vorgehen im SIPRI-Projekt auf die Zusammenarbeit von Praxis, Wissenschaft und Bildungsverwaltung ab, damit gemeinsam der Ist-Zustand der Primarschule analysiert und Verbesserungsvorschläge ausgearbeitet und erprobt werden konnten. ${ }^{171}$

\subsubsection{Die Lernziele der Primarschule: Anspruch und Wirklichkeit}

Da gibt es den Lehrplan 1907 und den anderen von 1977. Da gibt es nebeneinander den 5 seitigen Stoffkatalog, das 8oseitige Epos im Stil eines Erbauungsvortrags und den 300 seitigen Ordner mit Lernzielkatalogen, Stoffverteilungsplänen, methodischen Hinweisen und Lektionsskizzen. Da wird an einem Ort obligatorisch gefordert und am andern Ort unverbindlich angeregt. Da gibt es im Kanton A ein Fach für bestimmte Lernziele, welche im Kanton B diskret in vier andere Fächer eingebaut sind. ${ }^{172}$

Mit der Leitung des ersten SIPRI-Teilprojektes zu den Lernzielen der Primarschule wurde der Autor der oben stehenden Zeilen beauftragt, Anton Strittmatter, vormaliges Mitglied der Freiburger Arbeitsgruppe für Lehrplanforschung und zu diesem Zeitpunkt Leiter des Zentralschweizer Beratungsdienstes für Schulfragen. ${ }^{173}$ Bis im Juni 1982 konzipierte und diskutierte Strittmatter gemeinsam mit acht weiteren Mitgliedern, wie sie die Leitfragen ihres Teilprojektes umsetzen würden. Als Ausgangslage stand ihnen die Projektskizze der pädagogischen Kommission zur Verfügung, welche drei Schwerpunkte aufwies: ${ }^{174}$

I70 Salamin, I979, S. I 578 .

I7 I Schweizerische Konferenz der kantonalen Erziehungsdirektoren, Projekt SIPRI, I 98 I.

I72 Strittmatter, I979, S. I 582.

I73 Die EDK und die KOSLO als Ausarbeitende und Initiatoren des SIPRI-Projektes haben sich darauf verständigt, das Projekt fast ausschliesslich durch unentgeltliche Mitarbeit aller Teilnehmenden zu realisieren. Einzig für die beiden von der pädagogischen Kommission der EDK angestellten Projektleiter Jean-Pierre Salamin und Werner Heller wurden Teilzeitanstellungen ausgerichtet. Weitere Kosten waren von den Kantonen zu tragen, beispielsweise bei Freistellungen von Lehrpersonen für SIPRI oder bei weiterführenden Projektaufträgen im Dienste der beteiligten Schulplanungsdienste. Weiterführend Salamin, 1979; Anstellungsverträge der Projektleitung, I978, StALU, A I 270/926.

Strittmatter, I979, S. I 582. 
I. Auftrag der Primarschule gemäss Lehrplänen, 2. Bedeutung der Lehrpläne für den Unterricht, 3. Belastung des Primarschülers.

Wie das einleitende Zitat verdeutlicht, sah sich die Arbeitsgruppe vom ersten Teilprojekt vor keine einfache Aufgabe gestellt, zumal dem Unterricht auf der Primarstufe in jedem Kanton ein eigener Lehrplan zugrunde lag und die Lehrpläne nach wie vor gravierende Unterschiede aufwiesen. Der zu prüfende Auftrag der Primarschule gemäss Lehrplänen schien auf den ersten Blick ein ambivalenter zu sein.

In Bezug auf das zweite Ziel, die Überprüfung der Bedeutung von Lehrplänen für den Unterricht, stellte die Arbeitsgruppe ebenfalls fest, dass Lehrpläne bestimmten, die Untersuchungen erschwerenden «Randbedingungen» unterliegen würden: «Sie können obligatorisch oder fakultativ erklärt werden, Aussagen in Form von Handlungsalternativen oder von verpflichtenden Vorschriften enthalten» sowie «Ziele auf allgemeiner Ebene oder detailliert oder operationalisiert beschreiben». ${ }^{175}$ Wenn die Bedeutung der Lehrpläne für den Unterricht zu prüfen sei, so schloss die Arbeitsgruppe, gelte es jeweils die als Kontext verstandenen kantonalen Schulsysteme und die Gewohnheiten der Lehrpersonen mit zu berücksichtigen.

Zur Prüfung der Belastung von Primarschülern bemerkte Strittmatter bereits zu Projektbeginn: «Es gibt nicht die Belastung des Primarschülers, sondern es gibt wahrscheinlich ein grosses Bündel von Belastungsfaktoren, auf welche die verschiedenen Schüler sehr verschiedenartig mit Belastungssymptomen reagieren.» ${ }^{176}$ Die Arbeitsgruppe sah vor, in der Bearbeitung dieses dritten Schwerpunkts im Teilprojekt zwei Experten beizuziehen: zum Thema «Schülerbelastung» Prof. Siegfried von der Universität Bern und zum Thema Schule als «Stress-Ort» Prof. Meili von der Universität Zürich. ${ }^{77}$ Nichtsdestotrotz bereitete im weiteren Projektverlauf die Klärung von Belastungsfaktoren nach wie vor Schwierigkeiten, insbesondere weil kaum Forschung dazu vorzuliegen schien und demnach kaum auf Modelle zur Strukturierung der Diskussionen zurückgegriffen werden konnte. ${ }^{178}$

Die Arbeitsgruppe beschloss dann, ihre Arbeiten auf zwei Subgruppen aufzuteilen, eine zu «Lernzielen» und eine zweite zu «Belastungen». Auch aufgrund der Nachrangigkeit für die vorliegenden Fragestellungen wird auf eine weitere Analyse des Projektverlaufs der Subgruppe «Belastungen» verzichtet.

175 Projektskizze SIPRI, TP I, 3. I0. 1980, S. 7, StALU, A I $270 / 954$.

I76 Strittmatter, 1979, S. I 584 . Hervorhebung im Original.

I77 Arbeitsgruppe SIPRI, TP I, Protokoll der ersten Sitzung, 26. 2. 1980, S. 3, StALU, Ar 270/95 I.

I78 Zwischenbericht TP i, November 1980, StALU, A I 270/934. 
Wie dem Protokoll einer zu Beginn der SIPRI-Projektphase im Dezember 1979 anberaumten arbeitsgruppeninternen Diskussionsrunde zu entnehmen ist, war keinesfalls klar, in welche Richtung sich die Arbeiten zu entwickeln hatten oder welche Schwerpunkte gesetzt werden sollten. ${ }^{179}$ An erster Stelle wurde bemängelt, dass die vorgesehenen Aktivitäten der Arbeitsgruppe im Milizsystem zu stemmen seien. Zur Klärung dieser Fragen bedürfe es des Einsatzes von wissenschaftlichen Mitarbeitern. Dazu müsse Geld über ein ordentliches Budget beschafft werden. Zweitens bestand Unklarheit darüber, ob letztlich eine «Diagnose» ausreichend wäre oder auch «positive Vorschläge zur Entlastung der Lehrpläne» formuliert werden müssten. Die Arbeitsgruppe legte für sich fest, «dass den Kantonen mindestens Anleitungen zur ‘Entrümpelung〉 der Lehrpläne und zur Neubesinnung gegeben werden müssen. Solche Anleitungen wären Kriterien für die Elementarisierung des Primarschulstoffes, Rahmenempfehlungen für die Stundentafelgestaltung, Hinweise auf günstige Lehr- und Lernformen in den Fächern und auf Spielregeln zur Gestaltung der Hausaufgaben..$^{180}$ Aufgrund dessen war für die Arbeitsgruppe klar, dass es «eine Besinnung auf die Leitideen und Bildungsziele der Primarstufe» brauche. ${ }^{181}$ Hierzu nahm man sich vor, eine umfassende Analyse aller Schulfächer vorzunehmen und konkrete Empfehlungen von Lernzielen und Unterrichtsinhalten vorzulegen. So konnte man sich auch auf bereits geleistete Vorarbeiten abstützen, welche die Schweizerische Koordinationsstelle für Bildungsforschung (SKBF) eigens für das SIPRI-Projekt zusammengestellt hatte.

Die Projektübersicht der SKBF basierte auf den Daten ihrer Erhebung von 1972 sowie den seither laufend erfassten Bildungsforschungsprojekten in der Schweiz. Die für SIPRI aufbereiteten Forschungsangaben waren thematisch gemäss den vier SIPRI-Projektbereichen aufbereitet. Zum ersten Teilbereich «Auftrag der Primarschule gemäss Lehrplänen» wurden zunächst dreizehn Projekte aufgelistet, welche allesamt von der Freiburger Arbeitsgruppe durchgeführt worden waren. Es folgten i 8 weitere Projekte in der Verantwortung von neun verschiedenen Institutionen, bevor letztlich sechs Forschungsprojekte unter der Leitung des ZBS aufgeführt wurden. ${ }^{182}$

Diese Übersicht der SKBF verdeutlicht nicht nur die bereits in Kapitel zwei herausgearbeitete Dominanz der von den FAL-Mitarbeitern im Forschungsbereich Lehrpläne geleisteten Arbeiten. Die Übersicht steht in diesem Kontext sinnbildlich dafür, dass Forschungsarbeit der FAL in den Folgejahren wiederum als weithin sichtbarer Orientierungspunkt zur Rezeption empfohlen wor-

I 8 I Ebd.

I 82 Schweizerische Koordinationsstelle für Bildungsforschung, I98 I. 
den war, um «es den SIPRI-Mitarbeitern vor allem auch [zu] erleichtern, notwendige und gewünschte Kontakte zur Forschung herzustellen». ${ }^{83}$

Insgesamt zeigen die Ausführungen zu den Anfängen der ersten SIPRI-Arbeitsgruppe, dass die Analyse von Anspruch und Wirklichkeit der Primarschulen entlang bestimmten thematischen Leitfragen erfolgte und dazu die Lehrpläne in den Mittelpunkt gestellt wurden. Darüber hinaus ist das SIPRI-Teilprojekt in zweierlei Hinsicht von der Forschung der Freiburger Arbeitsgruppe eingeholt worden. Einerseits leitete mit Anton Strittmatter ein ehemaliges FAL-Mitglied die SIPRI-Arbeitsgruppe, welche kurzerhand entschied, Vorschläge zur «Entrümpelung» der Lehrpläne vorzulegen. Andererseits zeigte sich mit den Verweisen der SKBF auf SIPRI-relevante Forschung, dass im Lehrplanbereich nach wie vor nicht an den Konzepten der Curriculumforschung der FAL vorbeizukommen war. Es gab kaum nennenswerte Alternativen.

\subsubsection{Lehrpläne und Lehrplanentwicklung im SIPRI-Teilprojekt 1}

Die Analysen der lehrplan- und lernzielbezogenen Arbeiten des SIPRI-Projektes werden nachfolgend ihren drei thematischen Strängen entsprechend präsentiert. Die Arbeitsgruppe des Teilprojektes I hat gleichzeitig erstens Leitideen für die Primarschule als strukturierendes und koordinierendes Instrument der Lehrplanarbeit propagiert, zweitens didaktische Entwicklungstendenzen in den laufenden Lehrplanreformprojekten in den Kantonen erhoben und drittens die Frage verfolgt, inwiefern Lehrpläne im Unterricht wirksam sind.

\section{Leitideen in den Lehrplänen}

Es kommt nicht von ungefähr, dass Leitideen vor allem von Lehrplanschaffenden verlangt und ausgearbeitet werden. Einerseits machten sie die Erfahrung, dass die endlose Aneinanderreihung von weitgehend zusammenhanglosen Lernzielen (z. B. im Curriculum der Freiburger Arbeitsgruppe für Lehrplanforschung - FAL) einer Korrektur, oder besser eines pädagogisch-anthropologisch-didaktischen Rahmens bedurfte. Andererseits kann bei der Überarbeitung und Neufassung von Lehrplänen ohne bestimmte Orientierungshilfen keine zukunftsweisende Arbeit geleistet werden. ${ }^{1{ }^{8}}$

Im März 1982 trafen sich anlässlich des ersten SIPRI-Teilprojektes etwa 60 Erziehungswissenschaftler, Lehrpersonen und Mitarbeiter kantonaler Erzie-

I83 Ebd., S. 7 .

I84 A. Oggenfuss, 1983 . 
hungsdepartemente aus der ganzen Schweiz zur Auseinandersetzung «mit dem neuartigen Phänomen der Leitideen für die Primarschulen». ${ }^{185}$ Vorgängig, im Juni 198 I, wurde im Zuge eines Erfahrungsaustausches der kantonalen Beauftragten für Lehrplanrevisionen bereits vereinbart, ebenjene Leitideentagung dann im Kontext des SIPRI-Projektes auszubringen. ${ }^{86}$ Aus der Leitideentagung ist ein Tagungsband ${ }^{187}$ hervorgegangen, welcher umfassende Einblicke in die je kantonalen Bestrebungen, die geführten Debatten und die fachlichen Hintergründe gewährt.

Bemerkenswert an der Tagung im März 1982 ist zunächst das Programm. Die Liste der Referierenden umfasste Anton Strittmatter, den Leiter der SIPRIArbeitsgruppe von Teilprojekt eins, Joe Brunner mit einem Beitrag zu Leitideen in der Zentralschweiz und dem Tessin, Beat Mayer mit Ausführungen zu Funktion und Wirkung von Leitideen im Kanton Bern, Rudolf Künzli mit Einblicken in seine Vergleichsarbeit zum Leitideengebrauch verschiedener Kantone sowie zwei Kurzbeiträge von Carlo Jenzer und Werner Christen mit Erläuterungen zur Leitideenentwicklung in den Kantonen Aargau und Solothurn. Mit zwei Ausnahmen referierten also ausschliesslich ehemalige Mitarbeiter der Freiburger Arbeitsgruppe beziehungsweise Personen, die einen unmittelbaren Bezug zur Curriculumforschung der I970er-Jahre aufwiesen. ${ }^{188}$

In den Referaten und Podiumsdiskussionen wurden Leitideen weitgehend unkritisch als identitätsstiftende Elemente für die Lehrpersonen, strukturierende und koordinierende Instrumente im Lehrplanreformprozess oder gewinnbringendes Mittel zur Kommunikation vom Schulreformen gegenüber der Öffentlichkeit positioniert. ${ }^{189}$ Leitideen, so die Kernbotschaft über alle Beiträge hinweg, würden den bisherigen Ausdeutungsprozess der Zweckartikel in den kantonalen Unterrichts- und Schulgesetzen sichtbar, nachvollziehbar und letztlich einer breiten Mitsprache zugänglich machen. ${ }^{190}$ Vor dem Hintergrund des mit SIPRI verfolgten Anliegens, «eine Rückbesinnung auf die erzieherische Grundaufgabe, die Rolle der Primarschule» einzuleiten, wurden Leitideen an der Tagung als Vehikel positioniert, um die Debatten über zu ergänzende, zu erneuernde oder wegzulassende Bildungsinhalte bei der Lehrplanarbeit zu strukturieren. ${ }^{191}$

I 85 Schweizerische Konferenz der kantonalen Erziehungsdirektoren, I983, S. 3.

I 86 Arbeitsgruppe SIPRI, TP I, Protokoll vom 6. 5. I98 I, StALU, A I 270/95 I.

I 87 Schweizerische Konferenz der kantonalen Erziehungsdirektoren, I983.

I 88 In den I980er-Jahren befanden sich die Curriculumdebatten mit weitgehend denselben Akteuren in ihrer zweiten, latenten Phase. Weiterführend Höhener, 2018.

I89 Zusammenfassend die Einleitung in: Schweizerische Konferenz der kantonalen Erziehungsdirektoren, $1983, \mathrm{~S} .3$.

I90 A. Oggenfuss, 1983 , S. Io f.

I9I Ideensammlung zu einer Tagung Leitideen, Arbeitsgruppe des SIPRI-TP I, 24. I. I98 I, StALU, Ai 270/956. 
Im August 1983 legte der Kanton Bern eigens entworfene Leitideen für die Primar- und Sekundarschulen als Teil der neu erarbeiteten Lehrpläne vor. In den Kantonen Solothurn und Aargau genehmigten die Schulbehörden nach breiter öffentlicher Diskussion die je entwickelten Leitideen zur weiteren Verwendung in der Planung der noch laufenden Lehrplanarbeiten. ${ }^{192}$ In der Innerschweiz verabschiedete die IEDK im März I 984 die unter der Leitung des ZBS erarbeiteten Leitideen für die Volksschule als Empfehlung an die angeschlossenen Kantone. Die IEDK vermerkte dazu, es sei «zu hoffen, dass die Schulbehörden aller Stufen, Politiker, Lehrer, Eltern und alle an der Schule interessierten Kreise sie [die Leitideen] ernst nehmen und daraus die Konsequenzen ziehen». ${ }^{193}$

\section{Erhebung didaktischer Entwicklungstendenzen}

Auf Initiative von Anton Strittmatter setzte das SIPRI-Teilprojekt I einen zweiten Schwerpunkt in der Evaluation von «didaktischen Entwicklungstendenzen». ${ }^{194}$ Die mittels Fragebogen durchzuführende Erhebung beabsichtigte, eine umfassende Übersicht über die gegenwärtigen kantonalen Reformtendenzen zu schaffen. So sollten neue Entwicklungstendenzen in den Schulfächern und den fächerübergreifenden Lernbereichen konstatiert werden, um letztlich «zur Bestimmung künftiger Bildungsziele der Primarschulen und zur Findung von 〈Treffpunkten〉 auf interkantonaler Ebene» beizutragen. ${ }^{195}$ Es war also intendiert, aufgrund einer Erhebung der aktuellen Bildungsreformprojekte in den Kantonen ermitteln zu können, in welche Richtung sich Schule und Unterricht tendenziell entwickeln.

«Fortschreibung der FAL-Untersuchung» hatte Strittmatter schon beim Start von SIPRI I 979 auf dem ersten Übersichtsplan von Teilprojekt I festgehalten. ${ }^{196}$ Es bedürfe einer vergleichenden Erhebung von Zahl und Art der Fächer, der Stundendotationen und Einführungszeitpunkten aufgrund einer Analyse kantonaler Lehrpläne. Der Leiter der Arbeitsgruppe I hatte offenbar vor, an die Studien der FAL anzuknüpfen und einen Überblick über den Ist-Zustand im Lehrplan- und Lehrplanreformwesen zu erarbeiten.

$\mathrm{Zu}$ Beginn setzte sich die Arbeitsgruppe in internen Diskussionen mit der Unklarheit auseinander, was unter einer Reform überhaupt zu verstehen sei. Die Mitglieder debattierten die Frage: «Gehören z. B. nur offizielle, von den Erziehungsdepartementen initiierte Reformen dazu, oder auch Basisprojekte ver-

192 Mayer, 1984.

I93 Innerschweizer Erziehungsdirektorenkonferenz IEDK, I984, S. 2.

I94 Arbeitsgruppe SIPRI, TP I, Protokoll vom 26. 6. I980, StALU, A r 270/95 I.

I95 Projektentwurf SIPRI zu TP I, 29. 4. 198 I, S. 4, StALU, A I $270 / 956$.

I96 Ausschnitt aus dem Referat von A. Strittmatter, SIPRI-Orientierungstagung vom I. Io. I979, StALU, Ai 270/954. 
schiedener Lehrergruppen?» ${ }^{197}$ Es herrschte auch eine gewisse Unsicherheit in Bezug auf die Wahl der Erhebungsmethode. Das zunächst gewählte Vorgehen mittels Fragebogen zuhanden der kantonalen Verantwortlichen von Lehrplanund Lehrmittelprojekten schien unzuverlässig, weil es lokale, niederschwellige Reformprojekte in der Schulpraxis unberücksichtigt gelassen hätte. So entschieden sich die Mitglieder der Arbeitsgruppe, die Ermittlung der für eine Befragung geeigneten Personen den «kantonalen Verbindungsleuten ${ }^{198}$ aus dem SIPRI-Projekt zu überlassen, nicht ohne sie vorab anlässlich einer Tagung zu instruieren. Eine engere Definition des Reformbegriffs wurde nicht vorgenommen.

Im Juni 198 I legte die Arbeitsgruppe ihre Ergebnisse in Form eines Arbeitspapiers vor mit dem Titel «Didaktische Entwicklungstendenzen in der Primarschule - eine in allen Kantonen durchgeführte Erhebung zu aktuellen Reformprojekten in der Primarschule».199 Der Hauptautor des Papiers, Gianni Ghisla, betonte einleitend, dass hiermit bloss ein erster Schritt in der Analyse von Reformarbeiten und didaktischen Entwicklungstendenzen auf der Primarschulstufe erfolgt sei. Nun bedürfe es aufgrund dieser «Grunddaten und Materialien» einer «intensiven Diskussion [...] in enger Zusammenarbeit mit den kantonalen Verbindungsleuten», sofern «die Bestimmung gemeinsamer Schwerpunkte und Koordinationsansätze eines Lehrplanmodelles» ${ }^{200}$ ermöglicht werden sollte.

Welche Erkenntnisse präsentierten die vorliegenden Arbeitsergebnisse jener Umfrage unter den Kantonen? Zunächst wurde betont, dass einige Kantone unter Verweis auf anstehende Schulgesetzrevisionen oder die Unbedeutsamkeit ihrer Reformprojekte auf eine Rückmeldung und damit auf eine Teilnahme an der Umfrage verzichtet hätten. Einigen Raum nehmen ferner die Beschreibungen zur Einführung des Rahmenlehrplans für die Primarschulen in den französischsprachigen Kantonen seit 1972 ein. ${ }^{20 r}$ Schliesslich enthielt das Arbeitspapier nebst einer umfassenden tabellarischen Gesamtübersicht über

I97 Arbeitsgruppe SIPRI, TP I, Protokoll vom 26. 6. I980, StALU, A I 270/95 I.

I98 Per i 5. 5. 1979 schrieb die SIPRI-Projektleitung sämtliche Kantone an mit der Bitte, je einen «kantonalen Verbindungsmann» zu ernennen. In deren Aufgabenbereich fiel die Kommunikation zwischen der SIPRI-Gesamtleitung und den kantonalen Teilprojekten. Es war zudem ihre Aufgabe, bei der Besetzung von Arbeitsgruppen durch die Nennung geeigneter Personen mitzuhelfen. Vgl. Erster Zwischenbericht der SIPRI-Projektleiter, 31. I2. I979, StALU, A I 270/934.

I99 Gianni Ghisla, Didaktische Entwicklungstendenzen in der Primarschule, Juni I98 I, StALU, Ai $270 / 955$.

200 Ebd., S. 2.

20 I Die Commission interdépartementale romande de coordination de l'enseignement primaire leitete seit 1972 die Erarbeitung eines Rahmenlehrplans für die Primarschulen in den französischsprachigen Kantonen Bern, Freiburg, Neuenburg, Wallis und Waadt. Der 
die einzelnen Reformprojekte in den Kantonen ein Kapitel «Gesamtreformprojekte». Darin waren ausschliesslich Beschreibungen von Projekten zur Entwicklung von Leitideen für die Volksschule in den Kantonen Bern, Freiburg, Solothurn, Aargau und der Zentralschweiz aufgeführt. ${ }^{202}$ Ebendiese sind an der oben beschriebenen Tagung ein Jahr später dann präsentiert worden.

Die Erhebung didaktischer Entwicklungstendenzen im Zuge des ersten SIPRI-Teilprojektes könnte zusammenfassend als Pilotstudie bezeichnet werden, welche zunächst der Ermittlung von Trends in kantonalen Lehrplanreformprojekten diente, um in einem zweiten Schritt die Promotion ebendieser als koordinationswirksame Grundlage für künftige Revisionen zu begünstigen. Was auf den ersten Blick trivial erscheinen mag, setzt eine bestimmte Übersicht über die laufenden Reformprojekte in den Kantonen voraus, was keinesfalls gegeben war. Mit diesem SIPRI-Teilprojekt sind die Vorarbeiten der Freiburger Arbeitsgruppe wieder aufgenommen und weitergeführt worden. Dabei zeigte sich einerseits, dass zu Beginn der I980er-Jahre noch immer keine Übersicht über die laufenden Bildungsreformprojekte in den Kantonen bestand und die erforderliche Datenbeschaffung zäh war, andererseits wurde durch die Erhebung deutlich, dass etliche Kantone spätestens ab den frühen I980er-Jahren die Entwicklung von Leitideen als strukturierendes Konzept der Lehrplanarbeit aufgenommen haben. Im Zuge von Erhebungen, Tagungen und Informationsmaterial von SIPRI wurde gesamtschweizerisch diese Option der lehrplanbasierten Schulreform weiter beworben. Allerdings stellten sich die Mitglieder der ersten SIPRI-Arbeitsgruppe ab Projektbeginn die Frage, inwiefern Lehrpläne überhaupt im Schulunterricht wirken.

\section{Wirksamkeit von Lehrplänen im Unterricht}

Bereits in der ersten Ideensammlung für eine Projektskizze im Teilprojekt I von SIPRI ist notiert worden: «Der Auftrag der Primarschule hat bis jetzt vorwiegend in den Lehrplänen schriftlichen Niederschlag gefunden. Es soll daher

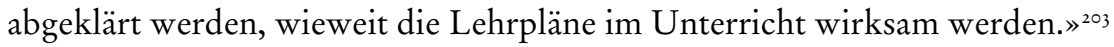
Das Ideenpapier legte sogleich zwei Anschlussfragen vor: Die erste fragte nach den Bedingungen dafür, dass ein Lehrplan auf den Unterricht wirke, die zweite fragte, inwiefern Lehrpläne als Grundlage für die Unterrichtsvorbereitung gelten würden. Aus diesen einleitenden Fragen wurde das Erkenntnisinteresse

bis in die frühen I980er-Jahre dauernde und auf die Sekundarstufe I ausgeweitete Prozess wurde begleitet und dokumentiert vom IRDP. Weiterführend Berthoud, 2016.

202 Gianni Ghisla, Didaktische Entwicklungstendenzen in der Primarschule, Juni 198 I, StALU, Ai 270/955.

203 Bedeutung des Lehrplanes für den Unterricht. Ideen zur Projektskizze, o. D., S. I, StALU, Ai 270/954. 
des Teilprojektes über die Wirksamkeit der Lehrpläne abgeleitet. Die Untersuchung sollte «aufzeigen, unter welchen Bedingungen des Lehrplans, der schulischen Situation und unter Einbezug der Lehrerpersönlichkeit ein Lehrplan grösseren oder kleineren Einfluss auf das Unterrichtsgeschehen hat». ${ }^{204}$

Die Konzeption der Studie wollte sich übrigens nicht auf die Unterrichtsvorbereitung beschränken, denn vielmehr «interessiert hier die Theorie des Unterrichts»». ${ }^{205}$ So listeten die Verfasser in der Ideenskizze unzählige Einflussfaktoren für schulischen Unterricht auf, um dann zu schliessen, man wolle darüber hinaus auch die Mechanismen für die jeweiligen Einflüsse auf Unterricht ergründen. Als Mittel der Datenerhebung war beabsichtigt, alle Lehrpersonen einer SIPRI-Projektschule in Interviews zu befragen.

Parallel zur Skizzierung von Ideen zur Erhebung der Einflussfaktoren von Lehrplänen auf schulischen Unterricht sind weitere methodische und theoretische Vorüberlegungen zur Erforschung der Wirksamkeit von Lehrplänen angestrengt worden. Über vier Seiten entfaltete ein entsprechendes Papier ein Modell der Wechselwirkung von Lehrplan und Unterricht. Eine bedeutende Rolle wurde der Lehrperson zugesprochen mit dem Verweis: «Bei der Person des Lehrers scheint mitentscheidend, ob er sich durch Identifikation (oder durch Gehorsam) dem geltenden Lehrplan verpflichtet fühlt.» ${ }^{206}$ Anders als in der Ideenskizzierung schlug dieses Papier aber eine Vergleichsstudie vor. Weil das Interesse für die Wirkung von Lehrplänen besonders auf die neuen Lehrpläne gerichtet sei, wäre es erstrebenswert, parallel dazu die Wirkung der älteren Lehrpläne ebenfalls zu erheben. Ferner lasse sich die Wirkung nicht allein mittels Befragungen von Lehrpersonen nachweisen. Es bedürfe zusätzlich einer Verarbeitung von Lehrplandokumenten und von weiteren Dokumenten zu kontextuellen Faktoren wie etwa Bedingungen der Unterrichtssituation oder kantonale Eigenheiten im Schulsystem. ${ }^{207}$

Anfang Oktober 1980 legte die Arbeitsgruppe des ersten Teilprojektes dann ihre Projektskizze vor. Basierend auf den Ideenskizzen wurde vorgeschlagen, erstens die Einflussfaktoren auf den Unterricht der Primarschulen und deren Mechanismen zu untersuchen, zweitens die Wirksamkeit der Lehrpläne unter Berücksichtigung der Persönlichkeiten der Lehrpersonen vorzunehmen. ${ }^{208}$ Im Projektentwurf vom April i98 I zuhanden der SIPRI-Projektleitung wurde dann deutlich unpräziser über die Vorhaben in Teilprojekt I informiert: «Durch gezielte Beobachtungen in den Kontaktschulen soll mehr über das

205 Ebd.

206 Wirksamkeit von Lehrplänen, 25. 9. 1980, StALU, A I 270/954, S. 2.

207 Ebd., S. 2-4.

208 Projektskizze SIPRI-TP I, 3. I0. I980, StALU, A $1270 / 954$. 
Zusammenspiel solcher Einflussgrössen (Lehrpläne, Lehrmittel, Selektionsnormen, Lehrerfortbildungskurse, strukturelle Weisungen usw.) bzw. über mögliche Bedingungen einer Aufwertung der Lehrpläne in Erfahrung gebracht werden.» ${ }^{209}$

Ein Jahr später war im dritten Zwischenbericht zu lesen, es würde «demnächst eine Untersuchung über die Wirksamkeit von Lehrplänen, Lehrmitteln und anderen Einflussgrössen in bezug auf die qualitative (didaktische) Veränderung der Schulwirklichkeit begonnen». ${ }^{20}$ Bis dahin scheint das Projekt nicht richtig Fahrt aufgenommen zu haben. Dennoch verblieb es auf einer Liste mit dem Titel «Was liefert das SIPRI-Teilprojekt I 〈Lernziele der Primarschule`?», welche zuhanden der pädagogischen Kommission der EDK im Juni 1982 abgefasst worden war. ${ }^{21 I}$ Der Liste war zu entnehmen, dass «in Verbindung mit dem CERI-Regionalseminar 1983 in Berlin» die «Durchführung und Anwendung von Studien über die relative Wirksamkeit von Lehrplänen» anstehe. ${ }^{212}$

Der Blick in den Tagungsband des Regionalseminars ${ }^{213}$ zum Thema Lehrplanentwicklung und Schulpraxis in Deutschland, Österreich und der Schweiz bestätigt durchaus die Präsenz von an SIPRI beteiligten Themen und Personen. Im Fokus der Debatten stand aber die Wirksamkeit von Lehrplänen ausschliesslich im Zusammenhang mit Leitideen. ${ }^{214}$ Zur angekündigten Untersuchung der Wirksamkeit von Lehrplänen oder zur Berücksichtigung von Merkmalen der Lehrpersonen war im Regionalseminar nichts dokumentiert. Auch in den Abschlussberichten, weiteren Publikationen zu SIPRI oder im Evaluationsbericht des Projekts blieben entsprechende Untersuchungsdokumentationen und der Begriff Wirksamkeit aus.

Über die Ursache für das Entfallen der Arbeiten zur Wirkung von Lehrplänen und damit einhergehenden Befragungen von Lehrpersonen anlässlich des ersten SIPRI-Teilprojektes kann nur spekuliert werden. Anknüpfend an die einleitenden Ausführungen zur Organisation von SIPRI wäre die plausibelste Ursache im Mangel an Ressourcen für ein solches Forschungsunterfangen zu suchen. Die an der betreffenden Arbeitsgruppe Beteiligten hatten teilweise Forschungserfahrung vorzuweisen und dürften realisiert haben, dass ihr Erkenntnisinteres-

209 Projektentwurf SIPRI zu TP i, 29. 4. I98 I, S. 4 f., StALU, Ai $270 / 956$.

2 I० Werner Heller, Jean-Pierre Salamin, SIPRI-Projekt. Dritter Zwischenbericht, März I982, S. 6, StALU, Ar $270 / 935$.

2 I I A. Strittmatter, Was liefert das SIPRI-Teilprojekt I «Lernziele der Primarschule»?, i I. 6. I982, StALU, Ar 270/956.

2 I 2 Ebd.

2I3 Beim CERI handelt es sich um ein Bildungsforschungszentrum der OECD. Es bearbeitete und förderte ab den späten 1960er-Jahren bildungspolitische Fragestellungen. Weiterführend Bürgi, 2017.

2 I 4 Vgl. dazu den Tagungsbeitrag von Mayer, 1984. 
se nicht beiläufig zu verfolgen war. Erst im Zuge des 33. nationalen Forschungsprogrammes zur Wirksamkeit der Bildungssysteme in der Schweiz ist die Frage nach dem Nutzen der Lehrplanarbeit und dem Gebrauch von Lehrplänen wieder bearbeitet worden. ${ }^{215}$ Das SIPRI-Teilprojekt I kann zumindest für sich in Anspruch nehmen, erste Vorüberlegungen dazu angestellt zu haben.

\subsubsection{Verbesserung der Primarschullehrpläne}

Es zeigt sich, dass Schulreformen heute meist von der Revision des Lehrplanes ausgehen. In vielen Kantonen sind Lehrplan-Revisionen in Gang resp. geplant. ${ }^{216}$

Eingefordert und mitkonzipiert von den schweizerischen Lehrerorganisationen, hatte das Projekt zur Überprüfung der Situation der Primarschule erneut die Lehrpläne, deren Funktion, Struktur und die scheinbar ideale Art und Weise ihrer Konstruktion zum Thema gemacht. Zu Beginn der I980er-Jahre hatten einige Kantone soeben ihre Lehrpläne neu geschrieben, andere standen erst am Anfang des Reformprozesses. Mit SIPRI war die Gelegenheit gegeben, die laufenden und anstehenden Arbeiten zu überblicken und zu bündeln. ${ }^{217}$

Für die thematische Ausrichtung und zur Bestimmung einzelner Teilprojekte spielte eine bedeutende Rolle, dass sich diejenigen Personen in der zugehörigen Arbeitsgruppe I zu Anspruch und Wirklichkeit der Primarschullernziele wiederfanden, die bereits über Sachverstand im Lehrplanwesen verfügten. Mit Anton Strittmatter, Joe Brunner und Beat Mayer prägten ehemalige Arbeitskollegen der Freiburger Arbeitsgruppe die Projektarbeiten. Sie publizierten bereits $1979 \mathrm{zu}$ Leitideen und zur Bestimmung von Lernzielen für die Primarschule. ${ }^{218}$ Dasselbe Konzept konnte nun mittels Tagungen und Publikationen einem breiteren Publikum beliebt gemacht werden, bestand eines der ersten Ziele von SIPRI doch darin, Lehrpersonen, Bildungsverwaltungsmitarbeitende und in der Erziehungswissenschaft Forschende in Austausch zu bringen.

Zur Beantwortung der Frage, wie und auf welcher Grundlage Lehrpläne verändert werden sollten, wurde auf die Wissensbestände der Arbeiten und Erfahrungen der FAL und des ZBS zurückgegriffen. Eine Weiterentwicklung dieser Vorarbeiten wäre angesichts des Projekts zur Wirksamkeit von Lehrplänen auf schulischen Unterricht beabsichtigt gewesen. Aber dafür stellte SIPRI als an

2 Is Adler und Künzli, I997; Bähr, I999; Künzli et al., I999.

2 I6 Didaktische Entwicklungstendenzen in der Primarschule, SIPRI Werkstattbericht 5, Bern I983, StALU, Ai 270/1025.

2 I7 Vgl. dazu auch Künzli, 2006.

2 I 8 Brunner, Eigenmann, Mayer und Schmid, 1979. 
Austausch interessiertes Grossprojekt nicht den geeigneten Rahmen bereit. Stattdessen bot es sich einmal mehr an, für den Lehrplan als geeignetes Instrument für Schulreformen zu werben sowie für zukünftige Lehrplanrevisionen Hilfsmaterial bereitzustellen.

\subsection{Planen, leiten und beraten: Bildungsengineering in Schulreformen}

Neue Ideen müssen sorgfältig und systematisch erprobt, die unerlässlichen Versuche vorausschauend geplant, seriös durchgeführt und gründlich ausgewertet werden. In diesem Zusammenhang kommt dem Gedanken- und Erfahrungsaustausch zwischen den an solchen Experimenten massgeblich Beteiligten eine wesentliche Bedeutung $\mathrm{zu}^{2}{ }^{219}$

Die Modelle und Konzepte der Curriculumforschung gewannen in bildungspolitischen Debatten der i970er-Jahre als problemlösungsorientierte $\mathrm{Pla}$ nungs- und Organisationskonstrukte Oberhand, so der abschliessende Befund aus Kapitel drei. Im vorliegenden vierten Kapitel wurde die Anschlussfrage verfolgt, inwiefern die Kantone in ihren Reformprojekten auf die Angebote der Curriculumforschung reagierten. Wurden im Zuge der Debatten Bildungsreformprojekte realisiert, die auf Wissensbestände der Curriculumforschung zurückgegriffen haben? Und wie können etwaige Rezeptionen charakterisiert und erklärt werden? Das einleitende Zitat von Urs Hochstrasser verdeutlicht bereits, dass den Leitern der Bildungsreformprojekte sowie den beteiligten Experten eine entscheidende Funktion in der Gestaltung der Reformprojekte zukam.

In den vier analysierten Bildungsreformprojekten waren je unterschiedliche Beweggründe im Spiel, die zu einer Überarbeitung von Lehrplänen führten. Im Kanton Basel-Stadt war der Beschluss zur Einführung der Koedukation auf der Sekundarstufe I umzusetzen, in der Zentralschweiz waren politische Forderungen nach mehr Durchlässigkeit und weitere schulstrukturelle Veränderungen das Reformmotiv. Im deutschsprachigen Teil des Kantons Freiburg stand das Streben nach innerkantonaler Harmonisierung der Lehrplanausführungen am Anfang der Reformprozesse, und SIPRI als bisher grösstes interkantonales Projekt der EDK ist einzuordnen als Reaktion auf die Emp-

219 Hochstrasser, I983, S. 5. Urs Hochstrasser war der damalige Leiter der Bundesabteilung für Wissenschaft und Forschung. Weiterführend Kapitel 3.3. 
fehlung, den Unterricht einer zweiten Landessprache auf der Primarschulstufe einzuführen.

Die Lehrpläne erfuhren eine grundlegende Veränderung, sowohl in ihrer Erscheinungsform als Dokument wie auch in Bezug auf ihre Funktion in den Reformprojekten. Im Zuge der kantonalen Lehrplanreformen wandelten sich die Lehrpläne von eher kurzen, überschaubaren Stoffsammlungen zu umfassenden Dokumenten. Sie enthielten zunehmend neue Elemente wie Leitideen, einen Stoffverteilungsplan, methodische und didaktische Hinweise als Anregungen für Lehrpersonen sowie Verweise auf Lernmedien oder weitere Hilfsmittel. Die schulischen Inhalte wurden neu in Form von Lernzielen festgehalten. Das SIPRI-Projekt hat diese Entwicklungen begünstigt, zumal der Vergleich unter den Kantonen zum reformwirksamen Argument aufgebaut worden war.

Die Kantone erhoben die Lehrpläne zum Instrument für die angestrebten Schulreformprojekte. Lehrpläne wurden nicht um ihrer selbst willen erneuert, nur in Ausnahmefällen wurde veraltete Fachlichkeit der Lehrpläne zum Argument. Vielmehr verfolgten die Kantone mit den initiierten Reformprojekten zunächst andere Zwecke, in erster Linie die Realisierung schulstruktureller Veränderungen. So bot sich dann die Gelegenheit, gleichsam über eine Veränderung der Lehrpläne in Bezug auf dessen Erscheinungsform und die Aufbereitung der Inhalte in Form von Zielen zu debattieren. Um die Festlegung von Leitideen wurde teilweise jahrelang gerungen, wie auch Lernziele insbesondere bei den Lehrpersonen nicht unumstritten waren.

Besonders die Erfahrungen in Projekten mit teilweise wenig ausgereiften theoretischen Konstrukten zur Lernzielformulierung oder mit planskizzenartigen Vorstellungen von partizipativer Lehrplanarbeit zeigten Rückwirkungen auf die Curriculumforschung. Dies ist nicht zuletzt dem von Beginn weg als Begleitforschung geplanten EBAC-Setting zuzuschreiben. Es sollte im Bereich der Lehrplanarbeit aber ein seltenes Beispiel bleiben. Bloss die im Zuge von SIPRI entstandenen, aber unbeantwortet gebliebenen Wirkungsfragen im Bereich von Lehrplänen können in ähnlicher Weise als Anstoss für eine spätere wissenschaftliche Bearbeitung stehen.

Zur Konzeption und Durchführung der einzelnen Lehrplanreformprojekte zogen die Kantone ausgewiesene Experten mit Forschungshintergrund und Bezügen zur Curriculumforschung bei und übertrugen ihnen Leitungsfunktionen in neu geschaffenen oder bestehenden Planungs- und Verwaltungsstellen. In Basel-Stadt prägte Guido Harder vom ULEF, in der Zentralschweiz der hierzu gegründete ZBS, in Freiburg die FAL und anlässlich des SIPRI-Projektes Anton Strittmatter die einschlägigen Entwicklungen. Sie planten und moderierten die Lehrplanarbeiten in den Kommissionen, legten die einzelnen Arbeitsschritte fest und griffen bei Bedarf auf weitere Experten wie etwa Karl Frey zurück. 
Die involvierten Experten und ihre Vorstellungen prägten die einzelnen Reformverläufe massgeblich. Sie übersetzten Begriffe und Modelle der Curriculumforschung freiburgischer Provenienz in möglichst praxistaugliche Verfahren und votierten an Kaderkursen, Weiterbildungen für Lehrpersonen, in pädagogischen Projektleitungskreisen oder gegenüber den Leitenden der Amtsstellen zugunsten von Leitideen, demokratischer Lehrplanarbeit oder der Entwicklung von Lernzielen. Kaum war jeweils der Startschuss für die Reformarbeiten gegeben, brachten sie die Erneuerung der Lehrpläne nach curriculumforschungsbasiertem Verständnis ins Spiel, entwarfen Pläne zur Vorgehensweise und legten dementsprechende Verfahren vor. Wenn die Lehrpläne schon überarbeitet werden sollten, dann nach den neusten Erkenntnissen der Forschung.

Das Vorgehen der beteiligten Experten folgte einer Systematik, welche dem Ingenieurwesen entliehen sein könnte: Sie analysierten eine bildungssystematische Problemstellung, konzipierten auf der Grundlage von Wissen aus der Curriculumforschung eine Projektbeschreibung und moderierten und leiteten dann in diesem Sinne die Arbeiten der Projektgruppen. Den Experten wurde also nicht die Analyse eines Problems zur Ausarbeitung einer Expertise anvertraut. Sie legten auch keine Variantenstudien vor. Sie präsentierten vielmehr Konzepte für Projekte zur Behebung der gegebenen Problemlagen. Ihre Arbeitsergebnisse sollten vorderhand keine weiteren Fragen aufwerfen, sondern die bestehenden beantworten. Die in Bildungsreformprojekten verpflichteten Experten betrieben Bildungsengineering. ${ }^{220}$

Für die einzelnen Reformverfahren in den Kantonen und im ersten SIPRI-Teilprojekt zog der Rückgriff auf Wissensbestände der Forschung der FAL mindestens drei unmittelbare Folgeerscheinungen nach sich: I. eine Steigerung der Komplexität der Verfahren, 2. einen intensivierten Einbezug der Lehrpersonen in die Prozesse der Erarbeitung der Lehrpläne, 3. eine unerwartet lange Dauer der Verfahren.

Im Zuge der analysierten Bildungsreformprojekte wurde eine Veränderung der kantonalen Lehrpläne hinsichtlich der Art und Weise der Fixierung von schulischen Inhalten angestrebt. Dies verursachte eine deutliche Steigerung der Komplexität der Lehrplanverfahren. Das Formulieren von Lernzielen oder das Ausarbeiten von Leitideen setzte curriculumtheoretisch begründete Vorgehensweisen voraus, welche für die beteiligten (Lehr-)Personen erläuterungsbedürftig waren und deshalb spezifische Weiterbildungen sowie den Einbezug von Experten notwendig machte. Darüber hinaus provozierte die

220 Dieser Begriff taucht teilweise auch im Kontext der angloamerikanischen Curriculumforschung auf. Dort wird er mit Referenz auf George A. Beauchamp allerdings zurückgebunden auf das Element der Planung. Vgl. Beauchamp, 1968. 
Einführung von Leitideen neue Reibungsflächen. Die Diskussion darüber, was eine gute Schule sei und welche schulischen Inhalte unmittelbar damit zu verknüpfen wären, erforderte Geschick in der Moderation entsprechender Aushandlungen und rief wiederum anforderungsreiche Theoriebezüge auf den Plan. Schliesslich arbeiteten oftmals mehrere Fachgruppen teilweise gleichzeitig und nur lose koordiniert an neuen Fachlehrplänen, während sich die Schulpraxis an mehrfach überarbeiteten, teilweise nur provisorisch in Kraft gesetzten Lehrplänen zu orientieren hatte. Nicht zuletzt wurde die Komplexität umfassender Lehrplanreformen zur Legitimationsformel für die Gründung verwaltungsnaher Schulberatungs- oder Planungsstellen mit entsprechend qualifiziertem Personal.

Die neu zu schreibenden Lehrpläne sollten im Sinne der Curriculumforschung zu umfassenden Dokumenten mit etlichen methodischen, didaktischen und unterrichtspraktischen Hinweisen erweitert werden, welche die Lernziele ergänzen würden. «Wer kann diese Arbeiten besser leisten als der Lehrer, der sich täglich mit dieser gesamten Problematik befasst? ${ }^{221}$ So lautete die von der Freiburger Arbeitsgruppe für Lehrplanforschung allseitig vertretene Position, und sie wurde zum Leitgedanken aller untersuchten Bildungsreformprojekte. Lehrplanarbeit wurde demokratisiert, mindestens vordergründig. Die Lehrpersonen lieferten fachgruppenspezifische Lernziele und weiterführende Hinweise und sie wirkten mit in der Formulierung von Lernzielen. Dabei waren sie aber immer eingebunden in curriculumtheoretische und fachliche Rahmungen, die ihnen von Experten in Weiterbildungen und Fachgruppenleitenden vorgegeben waren. Letztlich versprachen sich die FAL und diejenigen, welche sich auf ihre Konzepte bezogen, vom Einbezug der Lehrpersonen gleichwohl ein höheres Mass an Akzeptanz der Arbeitsresultate in der Schulpraxis. Damit lassen die untersuchten Bildungsreformprojekte eine deutliche Diskrepanz zu expertokratischen Curriculumprojekten in der Bundesrepublik nach Robinsohn erkennen und sind offensichtlich in der Tradition der FAL zu verorten respektive deren Rezeption angloamerikanischer Ansätze. ${ }^{222}$

Die Fallbeispiele aus der Zentralschweiz, dem Kanton Basel-Stadt und dem Kanton Freiburg stehen letztlich für Reformprojekte, welche zwar darauf angelegt waren, innert Kürze abgeschlossen zu sein, sich dann aber als langjährige Grossprojekte herausstellten. Man scheint sich zu Beginn der Verfahren nicht bewusst gewesen zu sein, inwiefern die Prozesse der Entwicklung der Lehrpläne in die Länge gezogen würden durch die beschriebenen komplexitätssteigernden Elemente, durch Vernehmlassungen oder weitere Demokrati-

221 Aregger, I971a, S. 239.

222 K. Frey et al., I97I; Goodlad, I964; Robinsohn, 1967. 
sierungsbemühungen. Bis in die frühen I $990 e r-J a h r e$ verabschiedeten deshalb die Kantone schrittweise einzelne Fachlehrpläne und operierten mit zunächst überprüfungsbedürftigen Provisorien. Im Grunde wurde das Vorhaben der Bildungsreform via Lehrplanreform jeweils aufgerieben durch unintendierte Folgeerscheinungen und zerfiel so in der Pluralisierung, in der Vielfalt einzelner, teilweise unübersichtlicher Lehrplanreformen. 


$$
\text { (name }
$$




\section{$5 \quad$ Netzwerke und die Dynamisierung pädagogischer Forschung}

Bildungspolitik, Bildungsverwaltung und die pädagogische Forschung sind eingebunden in Beziehungsgefüge, welche die gegenseitigen systematischen Grenzen relativieren und einen regen Wissensaustausch gewährleisten. Diese Erkenntnis aus der vorliegenden Untersuchung zu lehrplanspezifischen Entwicklungen in Forschung und Politik der Bildung um die I970er-Jahre könnte auf den ersten Blick als Gemeinplatz gelten. Vor dem Hintergrund der einleitend formulierten Fragestellung zur Entwicklung, Weiterentwicklung und Rezeption von pädagogischen Forschungserkenntnissen zu Curricula war jener Austausch aber von weitreichender Bedeutung für disziplinäre und bildungspolitische Entwicklungen wie auch für das teilweise schwierige Verhältnis zwischen den beiden Teilbereichen. Dazu wird im Folgenden eine Annäherung in sechs Punkten vorgenommen, um dann mit weiterführenden Überlegungen abzuschliessen.

I. Die wissenschaftliche Auseinandersetzung mit Lehrplänen in den I970erJahren war in der Schweiz unmittelbar verbunden mit den Tätigkeiten der Freiburger Arbeitsgruppe für Lehrplanforschung. Wissenschaftssoziologisch gesprochen hat sich die FAL unter der anfänglichen Leitung von Karl Frey als «Schule» formiert mit gemeinsamen Ansichten und Grundüberzeugungen dazu, worin die bedeutenden Fragestellungen der pädagogischen Forschung bestehen und mittels welcher methodischen Mittel diese zu bearbeiten wären. ${ }^{\mathrm{I}}$ Ein Merkmal der FAL bestand zunächst ganz wesentlich darin, ihren Namen als Programm positioniert und realisiert zu haben. Ab 1968 knüpfte sie an das international boomende, vorrangig angloamerikanisch geprägte Themenfeld der «curriculum studies» an und avancierte zum Orientierungspunkt in der Schweizer Curriculumforschung wie auch in den damit verbundenen Anwendungszusammenhängen. Erheblich zur Sichtbarkeit der FAL beigetragen haben die Konstitution als Arbeitsgruppe an der Universität Freiburg (Schweiz) mit bis zu zehn gleichzeitig engagierten Mitarbeitern und die thematische Kohärenz in den Artikeln und Monografien.

Ganz im Sinne der Forderung nach gesicherten Methoden zwecks einer «realistische[n] Wendung in der pädagogischen Forschung $»^{2}$ zielte die FAL auf

I Kuhn, 1967; Rieger-Ladich, 2009.

2 Roth, 1963, S. I09. 
zweierlei ab: erstens auf einen in ihrem Sinne wissenschaftlichen Entwurf zur Neubestimmung der Inhalte der Volksschule und zweitens auf eine Optimierung schulischen Unterrichts, etwa durch den Einbezug von Lernzielen in die Unterrichtsvorbereitung. Unter Berücksichtigung sozialwissenschaftlicher Vorgehensweisen erfragten die Mitglieder der FAL Präferenzen und andere Ansichten von Involvierten im Lehrplanbereich. Sie errechneten bereits computergestützt vermutete Zusammenhänge, um sie in ihren Publikationen zu diskutieren. Schliesslich konstruierten sie teilweise überkomplexe, theoretische Modelle als Referenzrahmen zur Selektion des künftig in Schulen zu vermittelnden Wissens. Insgesamt zeigen die Fragestellungen und Forschungsmethoden der Schweizer Curriculumforschung zugespitzt formuliert eine weitgehend einheitliche Stossrichtung: weg von geisteswissenschaftlichen Forschungstraditionen hin zur vergleichenden Perspektive, zur sozialwissenschaftlich ausgerichteten, anwendungsorientierten Forschung.

2. Aufgrund der Analysen gilt die FAL umso mehr als herausragendes Beispiel für eine Gruppe von Wissenschaftlern mit gemeinsamen bildungspolitischen Ambitionen, die gewillt war, ihre planvolle Absicht in die Tat umzusetzen, und sich auch nicht scheute, offensiv auf die Medien zuzugehen. Der Curriculumforschung war unter diesem Begriff zwar eine kaum zehnjährige Dauer beschieden. Mit der Auflösung der FAL I978 wurde in der Schweiz auch die Grundlagenforschung in dieser Domäne eingestellt. Die Anstrengungen ihrer Mitglieder erschöpften sich zeit ihres Bestehens aber bei weitem nicht in der reichlich geförderten Theoretisierung von Modellen zur Curriculumkonstruktion oder zur Curriculumreform. Sie hatten daneben gemeinsame Konzepte zur Bekannt- und Nutzbarmachung ihrer Forschungsleistungen ausgearbeitet und verfolgten eine, wie sich herausstellte, effiziente Verwertungslogik. So problematisierte die FAL zuerst die bestehenden Lehrpläne der Volksschule auf der Grundlage ihrer wissenschaftlich abgestützten Auffassung. Zugleich verwiesen ihre Vertreter öffentlichkeitswirksam in Zeitschriften für Lehrerinnen und Lehrer wie auch auf Konferenzen und Tagungen auf einen dringenden Handlungsbedarf, um letztlich ihre forschungsbasierten Konzepte und Modelle für eine Reform anzubieten.

Erstaunlicherweise schlug der FAL kaum nennenswerter Widerstand von «Gegenspielern» anderer «epistemic communities» oder wissenschaftlicher Schulen entgegen. Ihre Konzepte ernteten wohl Widerspruch an Tagungen, etwa von Forschern mit Bezügen zur kritischen Theorie oder mit Verankerung in der Psychologie oder der Wirtschaftspädagogik. In vorrangig bildungspolitisch initiierten Gremien waren ihre Standpunkte aber weitgehend unbestritten, was auch begründet sein dürfte in der Zusammensetzung der in den Gremien debattierenden Personen: Wenn Leute aus der Forschung zugegen waren, war die 
FAL gut vertreten. Es handelt sich aber kaum um ein singuläres Ereignis der intendierten Einflussnahme von Forschern auf die bildungspolitische Agenda. Mit Blick auf das erstarkende Forschungs- und Handlungsfeld der Sozialpädagogik in den I970er-Jahren könnten etwa bei Heinrich Tuggener vergleichbare Motive vermutet werden. ${ }^{3}$

3. Modelle und Konzepte der Curriculumforschung wurden zum ideologischen Gerüst, gewissermassen zur Leitidee der Lehrplanreformprojekte in den I970er- und I980er-Jahren. Vonseiten der Curriculumforscher ging ein wissenschaftlicher Impetus zur Reform der bis dahin gültigen Lehrpläne aus, welcher nicht zuletzt an der Ausgestaltung der zahlreichen kantonalen Lehrplanreformprojekte der Kantone in den Folgejahren abzulesen ist. In Bezug auf den inhaltlichen Aufbau der Lehrpläne, die Art und Weise der Fixierung schulischer Inhalte mittels Lernzielen, aber auch hinsichtlich der Verfahren ihrer Neubearbeitung stellte sich die Curriculumforschung als Referenzpunkt heraus. Wissen aus der Curriculumforschung hatte den Charakter von Regulierungswissen bei der Gestaltung von Reformprojekten angenommen.

Vonseiten der Bildungspolitik bestand sowohl auf der Ebene der Kantone wie auch auf der interkantonalen Ebene eine starke Nachfrage nach wissenschaftsbasierten Beratungs- und Entwicklungsangeboten im Lehrplanbereich, nicht zuletzt seit der Wille zur Koordination der kantonal stark divergierenden Schulsysteme mit dem Schulkonkordat vom Oktober 1970 zwar bekräftigt, aber von einigen Kantonen nur halbherzig umgesetzt worden war. ${ }^{4}$ Die von der EDK ausgegebene Strategie der Schulkoordination über Lehrplanreform ist von den Mitgliedern der FAL weitgehend mitgestaltet worden. Anknüpfend an die Dissertation von Karl Frey, entwarfen die Mitglieder der FAL anlässlich einer Auftragsstudie für die EDK ab Mitte der 1970er-Jahre erneut einen vergleichenden Überblick über die bestehende Lehrplanheterogenität in der Schweiz. Die Studie wurde zusammen mit ihren Vorschlägen über Form, Funktion und Inhalt künftiger Lehrpläne sowie mit konkreten Handreichungen für künftige Entwicklungsverfahren im Jahrbuch der EDKs publiziert und gleichsam zum Regelwerk für Lehrplanreformen bis in die I990er-Jahre.

An diesem exemplarischen Fall für ein Zusammenspiel von pädagogischer Forschung und bildungspolitischer Entwicklungsstrategie wird deutlich, dass die Positionierung des Gegenstandes der Curriculumforschung in bildungspoli-

3 Heinrich Tuggener war besonders in Forschung und Debatten zum Jugendmassnahmenvollzug und zu Jugendstrafuntersuchungen präsent. Analog zur FAL wurde der Forschungsbereich Tuggeners für die Bearbeitung gesellschaftlicher Fragen relevant, allerdings im sozialpädagogischen Politikfeld.

4 Manz, 20I I.

5 Jenzer et al., I 978. 
tischen Debatten auch einer Koinzidenz geschuldet war: Auf bildungspolitischer Seite lag ein offenkundiges Bedürfnis vor nach Lösungsvorschlägen zu aktuellen, bisweilen schulstrukturell begründeten Problemlagen. Dazu zählten die Einführung der Koedukation, die Abstimmung der Lehrpläne zwischen einzelnen Schulbezirken, die Revision der Lehrpläne im Zuge der Erweiterung der obligatorischen Schulzeit auf neun Jahre, wie auch die umfassende Überprüfung der Situation der Primarschulen anlässlich der Einführung des Frühfranzösisch. ${ }^{6}$ Wie auch immer das Problem umrissen wurde, als Dreh- und Angelpunkt seiner Bearbeitung wurde immer der Lehrplan definiert.

4. Die bildungspolitische Rezeption sozialwissenschaftlicher Deutungs- und Interpretationsangebote blieb nicht folgenlos.7 Als offensichtlichste und "greifbarste» Folge der Curriculumforschung könnte zunächst bezeichnet werden, dass die Lehrpläne als Dokument in den Kantonen Basel-Stadt, Freiburg und in der Zentralschweiz nach einer mehrjährigen Reformkaskade weit umfangreichere Ausmasse als die bisherigen Stoffsammlungen angenommen hatten. Über die veränderte Materialität hinaus implizierte die Berücksichtigung von Modellen und Konzepten der Curriculumforschung noch weitere Veränderungen, besonders im Feld der Bildungspolitik. Direkt abzulesen ist die als Versozialwissenschaftlichung zu beschreibende Entwicklung am vermehrten Bedarf an Expertinnen und Experten infolge einer erhöhten Komplexität des Gegenstandes sowie an einem damit erforderlich gewordenen Ausbau der Bildungsverwaltung. Insbesondere die Fallstudie zu Zentralschweizer Lehrplanreformen hat deutlich aufzeigen können, dass die in den späten I96oer-Jahren eingeleiteten Lehrplanreformen auf der Volksschuloberstufe in den frühen I970er-Jahren von den jüngsten Forschungserkenntnissen eingeholt wurden. Waren Lehrpersonen anfangs noch in der Überarbeitung von Lehrplänen weitgehend sich selbst überlassen, hatte bald eine mit wissenschaftlichem Personal durchsetzte Kommission die Aufsicht mit neuen Absichten übernommen. Die bisherigen Arbeiten wurden geflissentlich übergangen, stattdessen die Gründung des verwaltungsnahen Beratungsdienstes ZBS angeregt, seine Leitung einem ehemaligen Curriculumforscher übertragen und fortan die Lehrplanreformen weitgehend gemäss den Konzepten der Freiburger Arbeitsgruppe vorangetrieben. Das Einfangen von Rückmeldungen zu provisorischen Lehrplanentwürfen, die Vernehmlassungen zu Leitideen, besonders aber die Anleitung der Lehrpersonen zur Ausarbeitung von lernzielbasierten Lehrplänen waren inhaltlich voraussetzungsreich und generierten Bedarf an qualifiziertem Personal. Einige Mitglieder der FAL nutzten die Gelegenheit und wechselten auf

6 Vgl. im Einzelnen Kapitel 4.

7 Beck und Bonss, I985. 
dafür geschaffene Stellen in der Bildungsverwaltung. Wo jener Verwaltungsausbau politisch nicht opportun war, bedurfte es gleichwohl unterstützender Expertise, welche in Form von Beratungsmandaten in die Verfahren einfloss. Die bildungspolitische Rezeption sozialwissenschaftlicher Angebote aus der Feder der Curriculumforschung hat nebst den bisher diskutierten, eher systematisch-strukturellen Erscheinungen mindestens eine weitere, wenn auch seitens der Politik unintendierte Folge nach sich gezogen: ${ }^{8}$ Mit der Implementation von Lernzielen in Lehrpläne wurde die Planung und Durchführung von Unterricht vorderhand strukturierter, zumal Lernziele der Unterrichtsgestaltung einen engeren Rahmen vorgeben als die vagen, stichwortartigen Stoffsammlungen. Gleichzeitig eröffneten sich damit Möglichkeiten der standardisierten Überprüfung von Wissenserwerb über einzelne Schulklassen hinaus. ${ }^{9}$ Die pädagogische Forschung hat sich also weitere, in Zukunft bedeutsame Anwendungsgebiete geschaffen. In den Konzepten der Curriculumforscher war die Überprüfung von Lernzielen durchaus vorgesehen. Sie wurde als Errungenschaft und Verbesserung des Unterrichts propagiert, brachte den Forschenden aber auch einiges an Kritik ein. Die Lehrpersonen vermuteten Fremdsteuerung und Überwachung ihres Unterrichts, wie sie auch Unbehagen gegenüber einem lernzielbedingt steigenden Druck auf die Schülerinnen und Schüler äusserten. Curriculumforschung als bildungstechnologisches Erbe angloamerikanischer Herkunft gekoppelt mit Bezügen zu psychologischen Taxonomietheorien entpuppte sich erst Jahre später als fruchtbare Grundlage für Steuerungsabsichten der Politik mittels Schulleistungsmessungen. In keinem der untersuchten Bil-

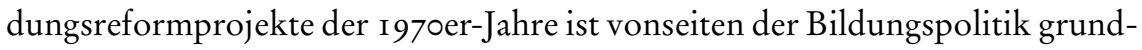
sätzliche Kritik an der Einführung von Lernzielen geäussert worden. Vielmehr geht aus den Analysen hervor, dass bildungspolitisch die Schulkoordination durch Lehrplanreform oder andere system- oder gesellschaftsbedingte Problemlagen im Fokus gestanden hatten, dass gegebenenfalls um die finale Fassung von Leitideen für die Volksschule gerungen wurde, nicht aber dass die Formulierung schulischer Inhalte in Form von Lernzielen verhandelt worden wäre. Mit der zunehmenden Präsenz von pädagogischen Forschungserzeugnissen in bildungspolitischen Debatten sind wohl Beratungsmandate und Expertisen einhergegangen, aber so weit wie Plumpe ${ }^{10}$ zu gehen und von neuer Unübersichtlichkeit in den bildungspolitischen Reformen zu sprechen - dieser Befund lässt

$8 \mathrm{Zu}$ unintendierten Folgeproblemen von Reformen weiterführend Lehmann und Imlig, 2018.

9 Diese Überlegung setzt natürlich die regelmässige Nutzung von Lehrplänen durch die Lehrpersonen zur Unterrichtsvorbereitung voraus, wobei die Forschung der FAL explizit auf dieses Ziel hinarbeitete. Vgl. Santini, I97I. 
sich im gegebenen Fall nicht stützen, im Gegenteil. Obwohl Lehrplanarbeit nach wie vor in kantonaler Zuständigkeit lag, schwenkten bald die meisten Kantone auf die neuen Konzepte zur Modernisierung ihrer Lehrpläne ein. Mindestens auf programmatischer Ebene gingen die Kantone inskünftig im Gleichschritt vor.

5. Die Frage nach der Bedeutung von Versozialwissenschaftlichung im Wechselspiel zwischen pädagogischer Forschung und Bildungspolitik stellt sich auch im umgekehrten Sinne: Inwiefern wirkte dieser Prozess auf die Forschung zurück? Mit Blick auf die Resultate aus der Untersuchung zur Finanzierung der Curriculumforschung in der Schweiz ist hervorzuheben, dass diese Forschungsrichtung aus zweierlei Quellen alimentiert wurde: einerseits vom Schweizerischen Nationalfonds, andererseits von kantonalen und privaten Geldgebern. Während der Nationalfonds drei Projekte im Bereich der Grundlagenforschung mitfinanzierte, waren alle übrigen im Bereich der anwendungsorientierten Forschung angesiedelt. Mit Tenorth kann die anwendungsorientierte Forschung in bestimmter Hinsicht als Vorläuferin der gegenwärtig teilweise kritisch diskutierten evidenzbasierten Bildungsforschung bezeichnet werden: ${ }^{11}$ Wissenschaftler forschten nach Vorgaben der Politik, waren angewiesen, handlungsrelevantes Wissen zu produzieren und ebenso Handlungsempfehlungen zu formulieren. Teile der Curriculumforschung sind also jener Verwertungslogik gefolgt und schufen sich mithin gravierende Normativitätsprobleme. Ein Ausweg aus dieser wissenschaftstheoretischen Bredouille bestand darin, sich von den theoretischen Modellen ein Stück weit zu verabschieden und stattdessen Leitideen als Referenzrahmen zur Bestimmung schulischer Inhalte beliebt zu machen. Der gesellschaftliche Konsens sollte der neue Referenzpunkt sein. Ein zweiter Umgang der Wissenschaftler mit dem Problem der Verwertungslogik wurde gefunden, indem praxis- oder schulnahe Curriculumentwicklung gestärkt und die Lehrpersonen bei der Formulierung schulischer Inhalte mehr eingebunden wurden. Es wurde mehr Verantwortung an die Schulpraxis delegiert. Schliesslich sind gegen Ende der I970er-Jahre vereinzelt selbstkritische Buch- und Zeitschriftenbeiträge erschienen, die auf das Legitimationsproblem schulischer Inhalte zu sprechen kamen und sich, wenig überraschend, von zukünftigen Resultaten der Lehr-Lern-Forschung Abhilfe versprachen. ${ }^{12}$

6. Teile der pädagogischen Forschung und die programmatische Ausrichtung von Bildungsreformen bezogen sich während einer kurzen Phase auf dieselben Wissensbestände, allerdings nicht zufällig. In den Anmerkungen über die Beziehung zwischen Wissenschaft und Politik für die zweite Hälfte des 20. Jahrhunderts erörtern Weingart, Lentsch und Ash die aufstrebende Rolle der Wis-

I I Für die Kritik vgl. auch Reichenbach, 20I7; Tenorth, 20I4a.

I2 Exemplarisch Eigenmann und Schmid, 1978. 
senschaft in der deutschen Politik. ${ }^{13}$ Sie verweisen auf das Grundproblem dieser Beziehung, wonach an das wissenschaftliche Wissen in Beratungszusammenhängen eine doppelte Anforderung gestellt wird: Wissen muss nach den Kriterien innerwissenschaftlicher Relevanz produziert werden und sich gleichzeitig auf die Kriterien politischer Relevanz beziehen. Die Curriculumforschung ist beiden Anforderungen nachgekommen. Als Forschungsbereich war sie während zehn Jahren breit rezipiert, und in bildungspolitischen Reformdebatten und Projekten wurden ihre Modelle und Konzepte als problemlösendes Planungs- und Organisationskonstrukt nachgefragt.

Die vorliegenden Analysen lassen darauf schliessen, dass Aushandlungsprozesse auf der intermediären Ebene zwischen Wissenschaft und Politik die bildungspolitische Themensetzung bestimmten und eine Rezeption von wissenschaftlichem Wissen begünstigten. ${ }^{14}$ Formate wie die Kongresse der Schweizerischen Gesellschaft für Bildungsforschung, die CERI-Seminare unter der Schirmherrschaft der OECD, Tagungen zur Sonderförderung der Erziehungs- und Bildungsforschung oder Projektgruppen im Bereich der Lehrerinnen- und Lehrerbildung führten Personen aus Bildungspolitik und Bildungsverwaltung zusammen mit Forschenden, die zur Bearbeitung der gegebenen Problemlagen eigene Konzepte und mithin Expertise anzubieten hatten. Innerhalb der so konstituierten Community bestimmte eine überschaubare Gruppe von Personen über die Themensetzung und den Verlauf der Debatten. Wie im Kapitel vier auch netzwerkanalytisch gezeigt, zählten einige Mitglieder der FAL mit zu den Schlüsselakteuren, welche ihre gemeinsam erarbeiteten Erkenntnisse aus der Forschung letztlich als bildungspolitisches Instrument profilieren und portieren konnten. Sie waren involviert in ein bildungspolitisches Establishment, welches in Bildungsreformprojekten und deren thematischer Ausrichtung den Takt schlug. ${ }^{\text {is }}$ Diese Personen nutzten die zur Verfügung stehenden Gremien und Podien nachhaltig, ihnen oblag die Besetzung von Stellen in Projekten und der Bildungsverwaltung mit neuem Personal. Sie waren bestens untereinander vernetzt.

Wenn inhaltliche Entscheidungen bei der Ausgestaltung bildungspolitischer Reformprojekte erklärt werden sollen, muss demnach die Tendenz zur Versozialwissenschaftlichung bildungspolitischer Sachfragen einhergehend mit der Verflechtung von Entscheidungsträgern und ihren Handlungsoptionen gedacht werden. Die von Terhart, Tillmann und Vollstädt nur angedeutete «wech-

I 3 Weingart, Lentsch und Ash, 2015.

I4 Halling und Fangerau, 2009; Holzer, 2009.

Is Establishments können sich auch innerhalb des wissenschaftlichen Feldes etablieren. So kommt eine fundierte Auswertung der ersten 25 Jahre «Zeitschrift für Pädagogik» zum Schluss, die Zeitschrift werde vom erziehungswissenschaftlichen Establishment dominiert. Vgl. Tenorth, 1986. 
selseitige[] Durchdringung» der Aufgabenbereiche von Bildungsforschung, Bildungspolitik, Bildungsverwaltung und Bildungspraxis ist aufgrund der vorliegenden Analysen wie folgt zu konkretisieren: ${ }^{16}$ Akteure der Forschung und der Verwaltung waren vor dem Hintergrund eines «technokratischen Wissenschaftsverständnisses ${ }^{17}$ wesentlich an der Aushandlung und Bestimmung von Zielsetzungen in Bildungsreformprojekte involviert, womit die systematisch definierte Arbeitsteilung zwischen den gesellschaftlichen Teilsystemen teilweise aufgehoben war. Wissenschaftler waren massgeblich in politischen Gestaltungsräumen aktiv, damit wurde Politik verwissenschaftlicht und umgekehrt Wissenschaft politisiert.

Dieser Befund wird gestützt durch den Rückgriff auf die These von Tillmann zum sich wandelnden Verhältnis von Pädagogik und Schulreform in Deutschland. ${ }^{18}$ Demnach habe sich die dortige pädagogische Forschung in den I970er-Jahren aus der politiknahen Forschung zurückgezogen und das Feld ausseruniversitären Instituten überlassen. In der Schweiz hat gleichermassen eine Verlagerung der politiknahen Forschung hin zu verwaltungsinternen Stellen wie dem ZBS stattgefunden. ${ }^{19}$ Dies könnte im gegebenen Zusammenhang auch interpretiert werden als institutionelle Einverleibung von Bereichen der Forschung durch die Verwaltung. ${ }^{20}$ Die Curriculumforschung wurde zu Verwaltungshandeln transformiert und damit politische Praxis. Die pädagogische Forschung hat sich damit ein neues Praxisfeld erschlossen.

Letztlich verweisen die Analysen zu Verfahren und Dimensionen des Wissensaustausches zwischen Forschung und Politik der Bildung auf ein symbiotisches Verhältnis. Forschungsergebnisse erreichen die Politik direkt oder über einen Umweg über die Medien. Eine direkte Folge davon ist laut Weingart et al. in der Form von gesteigerten Befindlichkeiten für Bedrohungsszenarien oder Verheissungen hinsichtlich vorliegender oder neu ausgemachter Problemlagen ersichtlich. Darüber hinaus würden Ergebnisse der Forschung einerseits wirksam im Agendasetting der Politik, provozierten andererseits weiteren Forschungsbedarf. ${ }^{21}$ Dasselbe Phänomen konnte in den I970er-Jahren bei der Curriculumforschung und ihrer bildungspolitischen Rezeption beobachtet werden, mit einem wesentlichen Unterschied: Während in etlichen Politikfeldern ein Häufung von Expertisen und Gegenexpertisen festgestellt werden konnte, ${ }^{22}$ gab

I6 Terhart, 200I, S. 27; Tillmann und Vollstädt, $200 \mathrm{I}$.

I7 Weingart, 2005, S. 20.

I8 Tillmann, 2008b.

I9 Criblez, 2016b.

20 Für die französischsprachige Schweiz hat Christina Rothen einen ebensolchen Befund herausgearbeitet: Rothen, 2016.

2 I Weingart et al., 20I 5 , S. i $\mathrm{f}$.

22 Dazu ausführlicher Strassheim, 20I4. 
es für die bildungspolitischen Entscheidungen bei Reformprojekten im Bereich der Lehrpläne keine Handlungsalternative in Form von Gegenexpertisen. Eine Revision der Lehrpläne gemäss den wissenschaftlichen Konzepten schien alternativlos, was auf die pädagogische Forschung zurückwirkte. Die Forschung in diesem Bereich war nun bildungspolitischen Erwartungen und Abhängigkeiten ausgesetzt. Drerup hat diesen Sachverhalt für die Bundesrepublik Deutschland wie folgt umrissen: «Zu der schon traditionellen wissenschaftstheoretischen, (alltags)praktischen und wissenschafts- sowie hochschulpolitischen Herausforderung der Erziehungswissenschaft tritt die bildungspolitische. ${ }^{23}$

Insgesamt förderten die Netzwerke(r) der Curriculumforschung eine Dynamisierung der pädagogischen Forschung. Dieser Prozess veränderte letztlich das Verhältnis zwischen der pädagogischen Forschung und den in sie gesetzten bildungspolitischen Erwartungen und forcierte gleichsam eine Wende hin zur empirischen Bildungsforschung. Die seit den I960er-Jahren in Schwang gekommenen Planungsbedürfnisse seitens der Politik waren im Zuge der Weltwirtschaftskrise ab Mitte der 1970er-Jahre vordergründig abgeschwächt. Gleichwohl bediente fortan die teilweise verwaltungsintern angesiedelte Bildungsforschung bildungspolitisch opportune Fragen und schickte sich an, für die Schulpraxis handlungsleitend zu werden. ${ }^{24}$ Jene Bildungsforschung konnte fortan als Teil der Erziehungswissenschaft wohl schulpraktische und damit gesellschaftliche Relevanz ihrer Resultate für sich beanspruchen. Ihre Relevanz war teuer erkauft mit dem Einverständnis, fortan in Abhängigkeit von politischen Themenkonjunkturen zu bestehen.

«Fragen sind nicht ursprünglich, nicht voraussetzungslos und sicherlich auch nicht vollkommen offen.»'s Diese grundsätzliche These zum geisteswissenschaftlichen Erkenntnisgewinn beschreibt im vorliegenden Zusammenhang prägnant die Einschränkung der thematischen Spielräume in der pädagogischen Forschung angesichts bildungspolitischer Erwartungen. Das Zitat betont zudem die Bedeutung von Wissensbeständen, welche die jeweiligen Forschungsfragen prädisponieren. Vor diesem Hintergrund scheint es unzureichend, den einleitend angeführten Problemkomplex zur Auswahl und Fixierung schulischer Inhalte allein mit einer Klärung von Kriterien, Verantwortlichen und Instrumenten abzustecken. Er ist zu ergänzen um eine Perspektive, welche die mit jener Frage beauftragten Forschenden, ihre Intentionen und ihr Wissen mit berücksichtigt.

$24 \mathrm{Zu}$ Wendungen und Windungen der deutschen Erziehungswissenschaft weiterführend K. Vogel et al., 2018.

25 Drews, Hubrich, Ludwig, Renker und Schütt, 2019, S. I8. 


$$
\text { (name }
$$




\section{Grafiken}

Grafik I: Verwendung des Schlagwortes «Curriculum» in deutschsprachiger Literatur 1960-1990

Grafik 2: SNF-Fördergelder Geisteswissenschaften I für Bildung und Erziehung

Grafik 3: Spätere Arbeitgeber der FAL-Mitglieder

Grafik 4: Schema zur Lernplanung

Grafik 5: Mitglieder der FAL in Projektgruppen der i 97oer-Jahre

Grafik 6: Alle an Projektgruppen Teilnehmenden der I 97oer-Jahre

Grafik 7: Alle Teilnehmende an Konferenzen und Seminaren der i 970er-Jahre

Grafik 8: Mitglieder der FAL in Projektgruppen, Konferenzen und Seminaren der I970er-Jahre

Grafik 9: Die Orientierungsstufe in der Zentralschweizer Oberstufenreform. Bericht der Kommission zur Gestaltung der Oberstufe

Grafik ıо: Zum Aufbau des Lehrplans. Lehrplan «Mensch und Umwelt», November 1983

Grafik I : Beteiligte Akteursgruppen im EBAC-Projekt 
AEPF

BIVO

CERI

EBAC-PS

EBAC-WS

EDK

ELEBU

ETH

FAL

IEDK

IPN

IRDP

KOSLO

LEMO

LUV

OECD

PK

SIPRI

SKBF

SLZ

SNF

ULEF

ZBS
Arbeitsgruppe für empirische pädagogische Forschung

Bildungsbedürfnisse der Volksschullehrer

Center for Educational Research and Innovation

Entwicklung und begleitende Analysen eines Curriculums für die

Primarschulen Deutschfreiburgs

Entwicklung und begleitende Analysen eines Curriculums der

Weiterbildungsschule Zug

Schweizerische Konferenz der kantonalen Erziehungsdirektoren

Expertentagung Lehrerbildung und Unterricht

Eidgenössische Technische Hochschule

Freiburger Arbeitsgruppe für Lehrplanforschung

Innerschweizer Erziehungsdirektorenkonferenz

Leibniz-Institut für die Pädagogik der Naturwissenschaften und

Mathematik

Institut romand de recherches et de documentation pédagogiques

Konferenz Schweizerischer Lehrerorganisationen

Expertenkommission «Lehrerbildung von morgen»

Lernzielorientierte Unterrichtsvorbereitung

Organisation für wirtschaftliche Zusammenarbeit und Entwicklung

Pädagogische Kommission der EDK

Projekt «Überprüfung der Situation der Primarschulen»

Schweizerische Koordinationsstelle für Bildungsforschung

Schweizerische Lehrerzeitung

Schweizerischer Nationalfonds

Institut für Unterrichtsfragen und Lehrerfortbildung

Zentralschweizer Beratungsdienst für Schulfragen 


\section{Quellen und Literatur}

\section{Ungedruckte Quellen}

\section{Staatsarchiv Basel (StABS)}

ED REG 5a, Knaben-Realschule 1930-1972, ab 1970/71 koeduzierte Klassen

\section{Staatsarchiv Luzern (StALU)}

A I 253 9, Protokolle des Erziehungsrates des Kantons Luzern

AI 270/926, Personelles - Besetzung der Projektleitungs- und Sekretariatsposten,

Verteilerschlüssel

A I 270/934, Information - Informationskonzept, Zwischenberichte, Informations-

broschüren

A I 270/935, Information - Informationskonzept, Zwischenberichte, Informations-

broschüren, Publikationsliste

Ar 270/95 I, Sitzungen SIPRI Teilprojekt I

A $270 / 954$, Verschiedene Unterlagen Projektierung SIPRI Teilprojekt I

AI 270/955, Erhebung in den Kantonen über didaktische Entwicklungstendenzen A $270 / 956$, Verschiedene Unterlagen SIPRI Teilprojekt I

Aı 270/1025, Leitideen für die Volksschule

A 810413 , Zentralschweizerischer Beratungsdienst für Schulfragen: Fortführung

Leitung (1975)

A 8104 I9, Zentralschweizerischer Beratungsdienst für Schulfragen: Tätigkeits-

berichte (1974-1976)

A 8 I 2 I67, Berichte Regierungsrat, Kommission zur Gestaltung der Oberstufe A 888 295, Oberstufenreform Luzern

A 696 380, Zentralschweizerischer Beratungsdienst für Schulfragen: Schaffung, Vorakten

A 69638 I, Zentralschweizerischer Beratungsdienst für Schulfragen: Schaffung der Stelle

A 696 382, Zentralschweizerischer Beratungsdienst für Schulfragen: ZBS-Informationen, Aufsichtskommission, Beschaffung Personal und Räumlichkeiten

\section{Staatsarchiv Zürich (StAZH)}

MM 3.I 30, Beschluss des Regierungsrates des Kantons Zürich Nr. 5384 vom 5. I I. 1970

\section{Erziehungsdirektion Basel-Stadt (EDBS)}

Reg. Nr. 430/I, Allgemeines, Lehrplan und Lehrziel, Pensen 


\section{Forschungsbibliothek Pestalozzianum}

FR HB I, Rechenschaftsbericht der Erziehungs- und Kultusdirektion des Kantons Freiburg für das Jahr 1968

LU HB 2, Bericht des Regierungsrates des Kantons Luzern an den Grossen Rat über die gesamte Staatsverwaltung in den Jahren 1970 und I97I

LU GD I, Lehrplan für die Sekundarschulen des Kantons Luzern, September 1972

\section{Institut für Unterrichtsfragen und Lehrerfortbildung Kanton Basel-Stadt (ULEF)}

Ordnung für das Institut für Unterrichtsfragen und Lehrerfortbildung des Kantons Basel-Stadt vom I4. 2. I 968

Guido Harder, Jahresbericht des Vorstehers, I97I

Karl Ingold, Zusammenfassende Orientierung über das Werkstattseminar für Lehrkräfte vom 21. 8. 1972 bis I. 9. 1972

Karl Frey, Schwerpunkte der Lehrplanreform für die Sekundarschulen, 7. I. I 974

Lehrplanüberarbeitung der Realschule im Kanton Basel-Stadt. Projektbeschreibung, I0. 5.1976

Jahresbericht 1979, H. P. Müller, 6. 8. 1979

Protokoll der Arbeitstagung der ULEF-Kommission, 23.-25. 6. 1983

\section{Staatsarchiv Freiburg (StAFR)}

DOA 2006/19, Schulkoordination I969-1975

DOA 2006/94, Lehrplan/Lehrertagungen

DOA 2006/I89, Lehrplankommission 1969-1978

\section{Archiv der Universität Zürich (UAZ)}

E.3.I.304, Professorendossier zu: Fend, Helmut, 1985-2006

\section{Dekanat der Philosophischen Fakultät der Universität Freiburg}

Schachtel 273, Section Pédagogie-Psychologie: Didactique générale, I967-I990

Schachtel 274, Section Pédagogie-Psychologie: Didactique de branche, I965-1993

\section{Archivalien der Freiburger Arbeitsgruppe für Lehrplanforschung (FAL)}

Tätigkeitsberichte und Jahresberichte des Forschungszentrums FAL, I97I-I978

Reglemente, Berichte, Konzepte und Protokolle

EBAC-Projekt, Arbeitsbericht Nr. 2, 1970

Reglement der FAL, Pädagogisches Institut der Universität Freiburg, 22. I. I97 I

K. Aregger, K. Frey, U. Isenegger, Die Curriculumreform der Sekundarschulen in der Innerschweiz, März I97 I 
FAL-Konzept zur Öffentlichkeitsarbeit im Rahmen der Schweizerischen Bildungspolitik, Januar 1972

Urs Isenegger, Jahresbericht der FAL zuhanden der Generalversammlung, 1972

Vorfragen an potentielle Auftraggeber für Projekte, Gutachten, Beratungen, Kurse, 28. 3. 1973

Informations- und Diskussionsnachmittag für Redaktoren und Journalisten zu

Fragen der schweizerischen Bildungspolitik, 7. 8. 1973

Protokoll der ordentlichen FAL-Generalversammlung I975, 4. 6. I975

Briefe

K. Schmid, Dringende Forschungsbedürfnisse in der Schweiz, 26. 5. 1970

C. Jenzer, Einladung an die Mitglieder der AG zur Tagung Lehrplangestaltung, Reform und Koordination, I 3. 5. 1976

\section{Privatarchiv von Karl Frey}

Karl Hecht an Karl Frey, I 2. 3., I 4. 4., I6. 7. 1970

Lebenslauf Karl Frey, i 5. 5. I980

\section{Periodika}

Bildungsforschung und Bildungspraxis, I979-1984

Deutschfreiburger Schulinformationen, 1978/79

Jahresberichte des Schweizerischen Nationalfonds, I967-1980

Jahresberichte der Konferenz der kantonalen Erziehungsdirektoren, I968-I 98 I

Lehrplan Informationen, I967, 1975-1977

Schweizerische Lehrerzeitung, I968-1979

Schweizer Schule, I968-1985

ZBS Information, I974-1988

\section{Gedruckte Quellen}

Achtenhagen, F. und Meyer, H. (197I). Einleitung. In: F. Achtenhagen und H. Meyer (Hg.), Curriculumrevision - Möglichkeiten und Grenzen (S. I I-2 I). München: Kösel.

Aebli, H. (196I). Grundformen des Lebrens. Ein Beitrag zur psychologischen Grundlegung der Unterrichtsmethode. Stuttgart: Klett.

Aebli, H. (I971). Entwicklungspsychologische Kriterien für die Auswahl von Curriculumsinhalten. In: K. Frey (Hg.), Kriterien in der Curriculumkonstruktion (S. 49-62). Weinheim: Beltz.

Aebli, H. (1985). Die Ausbildung der Ausbildner für die Lehrerbildung von morgen. Beiträge zur Lebrerinnen- und Lebrerbildung, 3 (I), 23-27.

Aregger, K. (I971a). Freiburger Lehrplanreform. EBAC-Bericht Nr. 5, Schweizer Schule, $58(6), 238 \mathrm{f}$. 
Aregger, K. (197Ib). Lehrplanreform als Grundlage der Schulreform. Schweizerische Lebrerzeitung, (50), I $824-\mathrm{I} 83 \mathrm{I}$.

Aregger, K. (1974). Lebrerzentrierte Curriculumreform. Planungsformen, Verlauf und organisatorische Modelle eines schulnaben Entwicklungsprojektes. Bern: Haupt.

Aregger, K. (1978). Schulinnovation als Arbeitsfeld des Lehrers. In: K. Aregger, U. P. Lattmann und U. P. Trier (Hg.), Lebrerbildung und Unterricht (S. 39-48). Bern: Haupt.

Aregger, K., Brunner, J. und Isenegger, U. (Hg.). (1972). Lebrplanreform als Grundlage der Schulreform. Freiburg: Kantonaler Lehrmittelverlag.

Aregger, K. und Gretler, A. (I983). Curriculumforschung in der Schweiz. In: K. Frey, H. Haft und U. Hameyer (Hg.), Handbuch der Curriculumforschung (S. 825-834). Weinheim und Basel: Beltz.

Aregger, K., Lattmann, U. P. und Trier, U. P. (Hg.). (1978). Lebrerbildung und Unterricht. Bern: Haupt.

Ausubel, D. P. (1968). The Psychology of Meaningful Verbal Learning. An Introduction to School Learning. New York: Grune \& Stratton.

Beauchamp, G. A. (I968). Curriculum Theory. Wilmette, IL: The Kagg Press.

Bloom, B. S. und Krathwohl, D. R. (1964). Taxonomy of Educational Objectives. The Classification of Educational Goals. New York: Longman.

Bossart, K. (1975a). Persönlichkeitsmerkmale von Lernzielformulierern. Eine empirische Untersuchung über Abnebmer, Experten, Ausbildner und Berufsträger als Formulierer von Lernzielen. Basel: Beltz.

Bossart, K. (1975b). Verfabren zur Lernzielerbebung. Entwicklung und Erprobung eines Modells zur Erbebung von Lernzielen für einen neuen Schultyp. Basel: Beltz.

Bruner, J. (I966). Toward a Theory of Instruction. Cambridge: Belknap.

Brunner, J. (1980). Die Erziehungsziele der Primarlehrer und Probleme ibrer empirischen Erhebung. Basel: Beltz.

Brunner, J., Eigenmann, J. und Mayer, B. (I977). Reformprozesse in der Freiburger Primarschule. In: E. Egger und B. Kehrli (Hg.), Bildungspolitik. Jabrbuch der Schweizerischen Konferenz der kantonalen Erziehungsdirektoren (S. 47-67). Frauenfeld: Huber.

Brunner, J., Eigenmann, J., Mayer, B. und Schmid, K. (1979). Die Leitideen. Ziele für die Primarstufe auf anthropologischer und didaktischer Grundlage. Zug: Klett \& Balmer.

Dolch, J. (1959). Lehrplan des Abendlandes. Zweieinhalb Jahrtausende seiner Geschichte. Ratingen: Henn.

Drerup, H. (Hg.). (1979). Wissenschaftstheorie und Wissenschaftspraxis. Probleme der Vermittlung zwischen metawissenschaftlichen Forschungsprogrammen und einer Praxis der Sozial-/Erziehungswissenschaft. Bonn: Bouvier.

Dubs, R. (1974). Praxisorientierte Curriculumforschung und Lehrplanarbeit. Schweizer Schule, 6I (4), 538-547. 
Dubs, R. (1976). Bildungsplanung und Schulreform als Problem der Erziehungswissenschaft. In: K. Widmer (Hg.), Bildungsplanung und Schulreform (S. 59-27). Frauenfeld: Huber.

Dubs, R., Metzger, C. und Hässler, T. (1973). Lebrplangestaltung und Unterrichtsplanung. Ein Modell für den Praktiker. Zürich: Verlag des Schweizerischen Kaufmännischen Vereins.

Edding, F., Jensen, S. N. und Ulshoefer, H. (1967). Internationales Seminar über Bildungsplanung. Berlin, 19.-28. Okt. 1966. Referate und Diskussionen. Berlin: Institut für Bildungsforschung in der Max-Planck-Gesellschaft.

Egger, E. und Blanc, E. (1977). Neuerungen im schweizerischen Schulwesen. Besonderbeiten und Tendenzen. Genf: Schweizerische Dokumentationsstelle für Schul- und Bildungsfragen.

Eigenmann, J. (1975). Sequenzen im Curriculum. Eine Studie zum Problem der Sequenzenbildung in der Curriculumentwicklung unter besonderer Berücksichtigung kognitionspsychologischer Theorien und des Lernzielansatzes. Weinheim: Beltz.

Eigenmann, J. und Schmid, K. (1978). Zwei Schwerpunkte aktueller Curriculumdiskussion. In: W.-R. Minsel (Hg.), Curriculum und Lebrplan (S. I68-I 87). München: Urban \& Schwarzenberg.

Eigenmann, J. und Strittmatter, A. (1972). Ein Zielebenenmodell zur Curriculumkonstruktion (ZEM). Beitrag zu einem standardisierten beuristischen Instrumentar zur Formulierung von Lernzielen. Fribourg: polykop. Skript.

Flitner, W. (1977). Verwissenschaftlichung der Schule? Zeitschrift für Pädagogik, $23(6), 947-955$.

Freiburger Arbeitsgruppe für Lehrplanforschung. (I97I). Is Thesen zur Bildungsplanung und Schulreform in der Schweiz. Schweizer Schule, 58 (I 2), 46 I.

Frey, K. (1968a). Der Bildungsauftrag der Realschule. Weinheim: Beltz.

Frey, K. (1968b). Der Lehrplan der Real-, Sekundar-und Bezirksschulen. Eine vergleichende Analyse zur Koordination und Neugestaltung der kantonalen Lehrpläne in der Schweiz. Weinheim: Beltz.

Frey, K. (I969a). Das Curriculum im Rahmen der Bildungsplanung und Unterrichtsvorbereitung. Die deutsche Schule, 6I, 270-28 I.

Frey, K. (1969b). Der Ausbildungsgang der Lebrer. Eine Modellanalyse des Unterrichts in den 52 Lebrerbildungsanstalten zum Zwecke der Curriculumreform. Weinheim: Beltz.

Frey, K. (1969c). Die Ausbildung der Lehrer an Volks-, Real-, Sekundar- und Bezirksschulen. Archiv für das schweizerische Unterrichtswesen, 54/196855/1969, 9-39.

Frey, K. (1969d). Lehrplananalyse als Teil der Curriculumforschung. International Review of Education, Is (I), 4-26.

Frey, K. (I970a). Die Taxonomien und das Curriculum. Paedagogica Europaea, 6, 50-72.

Frey, K. (I970b). Technisierung des Unterrichts? Schweizerische Lebrerzeitung, I I (28), I007-Iого. 
Frey, K. (1971). Theorien des Curriculums. Weinheim: Beltz.

Frey, K. (1975). Modelle diskursiver Legitimation. In: R. Künzli (Hg.), Curriculumentwicklung - Begründung und Legitimation (S. 103-1 29). München: Kösel.

Frey, K. (1978). Wissenschaftliche Disziplinen in der Lehrerbildung. In: K. Aregger, U. P. Lattmann und U. P. Trier (Hg.), Lebrerbildung und Unterricht (S. $25-$ 37). Bern: Haupt.

Frey, K., Horn, R., Isenegger, U., Lattmann, U. P., Rickenbacher, I. und Santini, B. (I97I). Eine Handlungsstrategie zur Curriculumkonstruktion. Zeitschrift für Pädagogik, I7 (I), I 2-29.

Frey, K. und Isenegger, U. (1972). Kriterien und Theorien in der Curriculumkonstruktion. In: S. B. Robinsohn (Hg.), Curriculumentwicklung in der Diskussion (S. 63-72). Stuttgart: Klett.

Frey, K. und Lattmann, U. P. (I 970). Effekte der Operationalisierung von Lernzielen. EBAC-Bericht 4. Freiburg: Universität, Pädagogisches Institut.

Gagné, R. M. (1965). The Conditions of Learning. New York: Holt, Rinehart and Winston.

Gehrig, H. (Hg.). (1970a). Die pädagogischen Disziplinen in der Lebrerbildung. Basel: Beltz.

Gehrig, H. (1970b). Einleitung: Problemstellung und Zielsetzung. In: H. Gehrig (Hg.), Die pädagogischen Disziplinen in der Lebrerbildung (S. 9-1 7). Basel: Beltz.

Goldschmid, M. (1977). Preface. In: Schweizerische Gesellschaft für Bildungsforschung. Erster Kongressbericht 1976 (S. 232-235). Lausanne: SGBF.

Goodlad, J. I. (1964). School Curriculum Reform in the United States. New York: Fund for the Advancement of Education.

Heckhausen, H. (1970). Lehrer 1980. Lebrerbildung für die künftige Schule. Düsseldorf: Bertelsmann.

Heiniger, U. (1974). Curriculumentwicklung an der Basis? Schweizer Schule, 6I (I), I3-I9.

Heiniger, U. (1977). Die Rolle des Lehrers, neu entdeckt. Didaktische Zentren als Marktplatz für Schulreform. Zug: Klett und Balmer.

Hochstrasser, U. (1983). Zum Geleit. In: J.-P. Meylan (Hg.), Evaluation von Innovationen im Bereich der Primarschule. Bericht über ein OECD/CERI-Seminar, Muttenz 198 I (S. 5). Bern: Haupt.

Horn, R. (1969). Ausbildung und Anstellung der Gymnasiallebrer. Eine vergleichende Dokumentation als Grundlage für die Reformplanung. Freiburg: Pädagogisches Institut Universität Freiburg.

Horn, R. (1972). Lernziele und Schülerleistung. Die Evaluation von Lernzielen im kognitiven Bereich. Weinheim: Beltz.

Huhse, K. (1968). Theorie und Praxis der Curriculum-Entwicklung. Ein Bericht über Wege der Curriculum-Reform in den USA mit Ausblicken auf Schweden und England. Berlin: Berlin.

Hutmacher, W. und Trier, U. P. (1976). Zusammenarbeit zwischen Forschung, Schulpraxis, Schulverwaltung und Politik: Problemstellungen, Modelle, Empfeh- 
lungen für künftige Arbeiten. In: K. Frey, K. Aregger und U. Hameyer (Hg.), Bedingungen und Modelle der Curriculuminnovation (S. I-23). Weinheim und Basel: Beltz.

Innerschweizer Erziehungsdirektorenkonferenz IEDK (Hg.). (1984). Leitideen für die Volksschule. IEDK.

Isenegger, U. (1972). Lernzielerhebung zur Curriculumkonstruktion. Methodenstudie für das BIVO-Projekt (Bildungsbedürfnisse der Volksschullebrer). Weinheim: Beltz.

Isenegger, U. (1973). Funktionen des Curriculum. Schweizerische Lebrerinnenzeitung, 77 (4), 93-107, https://doi.org/10.5 I69/seals-3 I 76 33

Isenegger, U. (1975). Einleitung. In: U. Isenegger und B. Santini (Hg.), Begriff und Funktionen des Curriculums (S. I0-19). Weinheim: Beltz.

Isenegger, U. (2016). Informationsgespräch zur FAL - Entstehung, Empirie und Weiterentwicklung der Curriculumforschung.

Isenegger, U. und Gehrig, H. (I97I). Bildungsbedürfnisse des Volksschullehrers. Zu einer Untersuchung im Kanton Zürich. Schweizer Schule, 58 (9), 3 I 8-325.

Jenzer, C. und Trier, U. P. (1978). Einleitung: Worum es in diesem Buch geht. In: C. Jenzer, J. Weiss und A. Strittmatter (Hg.), Schulkoordination über Lehrplanreform. Jabrbuch der Schweizerischen Konferenz der kantonalen Erziebungsdirektoren (S. I I-I 2). Frauenfeld: Huber.

Jenzer, C., Weiss, J. und Strittmatter, A. (Hg.). (1978). Schulkoordination über Lehrplanreform. Jahrbuch der Schweizerischen Konferenz der kantonalen Erziebungsdirektoren. Frauenfeld: Huber.

Kägi, E. (5. I I. 1974). Sonderförderung der Erziehungs- und Bildungsforschung. Nene Zürcher Zeitung.

Kaiser, L. (1970). Die Fortbildung der Volksschullehrer in der Schweiz. Eine empirische Untersuchung der Situation in den 25 Kantonen der Schweiz, bei den Volksschullehrern und den Bezirksinspektoren des Kantons Luzern (Bd. 6). Weinheim; Basel: Beltz.

Knab, D. (1969). Curriculumforschung und Lehrplanreform. Nene Sammlung, 9 (2), I69-1 85 .

Konferenz der kantonalen Erziehungsdirektoren. (1970). Jahresbericht der Konferenz der kantonalen Erziebungsdirektoren 1968/69. Zug, Genf.

Konferenz der kantonalen Erziehungsdirektoren. (1974). Jabresbericht der Konferenz der kantonalen Erziehungsdirektoren 1973/74. Zürich, Genf.

Konferenz der kantonalen Erziehungsdirektoren. (1975). Jahresbericht der Konferenz der kantonalen Erziebungsdirektoren 1974/75. Zürich, Genf.

Konferenz der kantonalen Erziehungsdirektoren. (1977). Jabresbericht der Konferenz der kantonalen Erziehungsdirektoren 1976/77. Zürich, Genf.

Lattmann, U. P. (I97Ia). Kriterien in der Curriculumkonstruktion. Weinheim: Beltz.

Lattmann, U. P. (I97 Ib). Lernziele und Unterrichtsvorbereitung. EBAC-Projekt, Bericht 7. Basel: Beltz.

Lattmann, U. P. (1972). Unterrichtsvorbereitung. Hitzkirch: Comenius-Verlag. 
Lattmann, U. P. (1978). Qualifizierung des Lehrers für das Unterrichten. In:

K. Aregger, U. P. Lattmann und U. P. Trier (Hg.), Lebrerbildung und Unterricht (S. 97-106). Bern: Haupt.

Lattmann, U. P. (2015). Informationsgespräch zur FAL. Entstehung, Empirie und Weiterentwicklung der Curriculumforschung.

Mager, R. F. (1965). Lernziele und programmierter Unterricht. Weinheim: Beltz.

Mayer, B. (I984). Leitideen im Lehrplan und ihre Bedeutung für die Schulpraxis. In: Schweizerische Konferenz der kantonalen Erziehungsdirektoren, Bundesministerium für Unterricht und Kunst und Bund-Länder-Kommission für Bildungsplanung und Forschungsförderung (Hg.), Lebrplanentwicklung und Schulpraxis in Deutschland, Österreich und der Schweiz. OECD/CERISeminar Berlin 1983 (S. 72-85). Bonn: Köllen.

Meyer, H. (I97I). Das ungelöste Deduktionsproblem in der Curriculumforschung. In: F. Achtenhagen und H. Meyer (Hg.), Curriculumrevision. Möglichkeiten und Grenzen (S. I06-I32). München: Kösel.

Meylan, J. P. (1979). Die Überprüfung der Situation der Primarschule (SIPRI). Schweizer Schule, 66 (I 8), 626-630.

Möller, C. (1969). Technik der Lehrplanung. Berlin, Weinheim, Basel: Beltz.

Moser, H. (I97 I). Technik der Lernplanung: Curriculumforschung und Ideologie. Zeitschrift für Pädagogik, I7 (I), 55-74.

Moser, H. (Hg.). (1973). Probleme der gegenwärtigen Erziebungswissenschaft II. Vorlesungen zum Funkkolleg Erziehungswissenschaft. Zürich: Pädagogisches Institut der Universität Zürich.

Moser, H. (1974). Handlungsorientierte Curriculumforschung. Überlegungen zum gegenwärtigen Stand der Curriculumdiskussion. Weinheim: Beltz.

Moser, H. J. (1980). Entstebung kantonaler Curriculumreformen. Überprüfung theoretischer Erklärungsansätze anhand von Fallstudien. St. Gallen: ok Matterhorn AG.

Müller, F. (Hg.). (1975). Lebrerbildung von morgen. Grundlagen - Strukturen Inhalte. Bericht der Expertenkommission «Lehrerbildung von morgen» im Auftrag der Schweizerischen Konferenz der kantonalen Erziebungsdirektoren. Hitzkirch: Comenius-Verlag.

Müller, F. (1985). Professionalisierung - Eine Rückschau auf die Wurzeln und Wesen des LEMO-Berichts. Beiträge zur Lehrerbildung, 3 ( I), I I-I 8.

Muntwyler, M. (1973). Was lange währt, macht gar nichts gut. Schweizerische Lehrerzeitung, (5), 2 I0-2 I I.

Niedermann, A. (1977). Formative Evaluation. Entwicklung und Erprobung einer Evaluationskonzeption für eine Modellschule. Weinheim Basel: Beltz.

Oggenfuss, A. (1983). Was sind Leitideen? In Schweizerische Konferenz der kantonalen Erziehungsdirektoren. Pädagogische Kommission (Hg.), Leitideen für die Primarschule: kantonale und regionale Konzepte. Diskussionsergebnisse einer Tagung über Leitideen [Freiburg], März I982 (S. 9-I4). Genf: Schweizerische Konferenz der kantonalen Erziehungsdirektoren. 
Oggenfuss, F. (1984). Leitziele der Schule und Lehrplanreform. In: Schweizerische Konferenz der kantonalen Erziehungsdirektoren, Bundesministerium für Unterricht und Kunst und Bund-Länder-Kommission für Bildungsplanung und Forschungsförderung (Hg.), Lebrplanentwicklung und Schulpraxis in Deutschland, Österreich und der Schweiz. OECD/CERI-Seminar Berlin 1983 (S. 55-59). Bonn: Köllen.

Ohne Autor (1976). Nationale Forschungsprogramme. Schweizerische Bauzeitung, 94 (2 I), 286.

Oswald, F. (1984). Zielansatz der Lehrplanreform - Anspruch und Wirklichkeit. In: Schweizerische Konferenz der kantonalen Erziehungsdirektoren, Bundesministerium für Unterricht und Kunst und Bund-Länder-Kommission für Bildungsplanung und Forschungsförderung (Hg.), Lebrplanentwicklung und Schulpraxis in Deutschland, Österreich und der Schweiz. OECD/CERISeminar Berlin 1983 (S. 42-54). Bonn: Köllen.

Preisig, E. (1980). Das erzieherische Belobnungs- und Bestrafungsverhalten. Eine empirische Untersuchung zur Befragung sowie zu den Auswirkungen und Bedingungen erzieherischer Bekräftigung. Bern: sn.

Regierungsrat des Kantons Bern. Verordnung über die Ausbildung und Prüfung von Lehrern und Sachverständigen der Erziehungs- und Bildungswissenschaften (I974).

Rickenbacher, I. (1970). Lehrplanreform an Volksschulen. Schweizerische Lebrerzeitung, i I 5 (20), 663-675.

Rickenbacher, I. (1972a). Curriculumreform als Ansatz zur Schulreform. Schweizer Schule, 59 (2), 59-65.

Rickenbacher, I. (1972b). Entwicklungsplan der Schulen im Kanton Schwyz. Liebefeld/Bern: Lang.

Rickenbacher, I. (I974). Der Lehrer als Objekt wissenschaftlicher Untersuchungen. Schweizer Schule, 6I (4), 547-550.

Rickenbacher, I. (20I6). Informationsgespräch zur FAL. Entstehung, Empirie und Weiterentwicklung der Curriculumforschung.

Robinsohn, S. B. (1967). Bildungsreform als Revision des Curriculum. Luchterhand.

Robinsohn, S. B. (I971a). Ein Struktur-Konzept zur Curriculum-Entwicklung. In: F. Achtenhagen und H. Meyer (Hg.), Curriculumrevision-Möglichkeiten und Grenzen (S. 57-74). München: Kösel.

Robinsohn, S. B. (I97 Ib). Zur gegenwärtigen Curriculumdiskussion, 24 (Bildung und Erziehung), $32 \mathrm{I}-326$.

Robinsohn, S. B. (Hg.). (1972). Curriculumentwicklung in der Diskussion. Stuttgart: Klett.

Roller, S. (1975). Regards sur la recherche éducationnelle en Suisse en I975. Neuchâtel: IRDP.

Roth, H. (1963). Die realistische Wendung in der Pädagogischen Forschung. Die Deutsche Schule, 55 (3), I09-I I9.

Roth, H. (1968). Stimmen die Deutschen Lehrpläne noch? Die Deutsche Schule, $60(2), 69-76$. 
Ruhstaller, P. R. (1977). Nachruf Pater Ludwig (Hans) Räber. Einsiedeln: Stiftsschule Einsiedeln, www.gwick.ch/M63/nekrolog/PLR.pdf

Salamin, J. P. (1979). SIPRI-SIPRI-SIPRI. Überprüfung der Situation der Primarschulen. Schweizerische Lehrerzeitung, 44 (Sondernummer), I 577-I 579.

Santini, B. (1970). Die Bedeutung des Curriculums in der inneren und äußeren Bildungsplanung. Schweizer Schule, 57 (14-1 5), 526-528.

Santini, B. (I97I). Das Curriculum im Urteil der Lebrer. Eine empirische Untersuchung. Weinheim: Beltz.

Schweizerische Gesellschaft für Bildungsforschung. (1988). Entwicklungsplan der schweizerischen Bildungsforschung. Bildungsforschung und Bildungspraxis, Io (Sondernummer).

Schweizerische Konferenz der kantonalen Erziehungsdirektoren (Hg.). (I983). Leitideen für die Primarschule. Kantonale und regionale Konzepte. Diskussionsergebnisse einer Tagung über Leitideen. Genf: Schweizerische Konferenz der kantonalen Erziehungsdirektoren.

Schweizerische Konferenz der kantonalen Erziehungsdirektoren (Hg.). ( 985 ). Lebrplan-Lebrplanentwicklung. Dossier. Bern: Sekretariat EDK.

Schweizerische Konferenz der kantonalen Erziehungsdirektoren und Conférence suisse des directeurs cantonaux de l'instruction publique, Projet SIPRI. (I98 I). SIPRI-Werkstattbericht. Genf: Schweizerische Konferenz der kantonalen Erziehungsdirektoren.

Schweizerische Koordinationsstelle für Bildungsforschung. (1973). Tätigkeitsbericht September I97I - August 1973. Aarau: SKBF.

Schweizerische Koordinationsstelle für Bildungsforschung. (1975). Bericht über die Arbeitstagung "Die Bildungsforschung im Rabmen des Forschungsberichtes des Schweizerischen Wissenschaftsrates" Oktober 1974. Aarau: SKBF.

Schweizerische Koordinationsstelle für Bildungsforschung. (I98 I). Schweizerische Forschungs- und Entwicklungsprojekte im Bereich der obligatorischen Schulzeit. Ein Beitrag der Schweizerischen Koordinationsstelle für Bildungsforschung an das Projekt SIPRI (Überprüfung der Situation der Primarschule) der Schweizerischen Erziebungsdirektorenkonferenz. Aarau: Schweizerische Koordinationsstelle für Bildungsforschung.

Schweizerischer Wissenschaftsrat. (1973). Schlussbericht Bildungsforschung. Ermittlung über die dringlichen Forschungsbedürfnisse der Schweiz. Bern: Schweizerischer Wissenschaftsrat.

s. n. (I972). SUMACUCO. Ein Interview im Jahre 2002 - auch eine Reaktion zu SLZ I I/I972. Schweizerische Lebrerzeitung, (I 5), 59I.

s. n. (I973). Aufgabenbereiche der Pädagogischen Kommission. Schweizer Schule, 60 (34), 1032-1037.

Stricker, H., Isenegger, U. und Santini, B. (I978a). Form und Inhalt künftiger Lehrpläne. In: C. Jenzer, J. Weiss und A. Strittmatter (Hg.), Schulkoordination über Lehrplanreform. Jabrbuch der Schweizerischen Konferenz der kantonalen Erziebungsdirektoren (S. 65-84). Frauenfeld: Huber. 
Stricker, H., Isenegger, U. und Santini, B. (I978b). Vorschläge betreffend Minimalanforderungen an die Form von Lehrplänen. In: C. Jenzer, J. Weiss und A. Strittmatter (Hg.), Schulkoordination über Lehrplanreform. Jabrbuch der Schweizerischen Konferenz der kantonalen Erziehungsdirektoren (S. 84-88). Frauenfeld: Huber.

Strittmatter, A. (1973). Curriculumforschung - Weg in Entmenschlichung und Bildungsdiktatur? Schweizerische Lebrerzeitung, (2), 5 I-56.

Strittmatter, A. (1975). Der Beitrag der Hochschulen zur Lehrerbildung und deren Reform. In: U. Isenegger, I. Rickenbacher und A. Strittmatter (Hg.), Reformbedingungen der Lebrerbildung in der Schweiz. Über die Notwendigkeit und die Möglichkeiten einer dauernden Reform (S. 55-74). Basel: Beltz.

Strittmatter, A. (1978a). Die Mitarbeit der Wissenschafter und Lehrer an der Lehrplanentwicklung. In: C. Jenzer, J. Weiss und A. Strittmatter (Hg.), Schulkoordination über Lebrplanreform. Jabrbuch der Schweizerischen Konferenz der kantonalen Erziebungsdirektoren (S. 84-88). Frauenfeld: Huber.

Strittmatter, A. (1978b). Grundsätze für die Lehrplanentwicklung. Schweizer Schule, 65 (I I), 380-383.

Strittmatter, A. (1978c). Lehrerbildung für die Orientierungsstufe: Empfehlungen einer Expertenkommission der Innerschweizer Erziehungsdirektorenkonferenz. Schweizer Schule, 65 ( I-2), 5-7.

Strittmatter, A. (1978d). Vorschläge zum Verfahren der Lehrplanentwicklung und zur Zusammenarbeit der Kantone. In: C. Jenzer, J. Weiss und A. Strittmatter (Hg.), Schulkoordination über Lehrplanreform. Jabrbuch der Schweizerischen Konferenz der kantonalen Erziebungsdirektoren (S. I I9-I 22). Frauenfeld: Huber.

Strittmatter, A. (1979). Lernziele der Primarschule: Anspruch und Wirklichkeit. Schweizerische Lebrerzeitung, 44 (Sondernummer), I $582-1585$.

Strittmatter, A. und Winiger, X. (1978). Der Kommissionsbericht im Abriss.

Schweizer Schule, 65 (I-2), 7-24.

Taba, H. (1962). Curriculum Development. New York: Harcourt, Brace \& World.

Treml, A. (I97I). Karl Frey: Theorien des Curriculums. Zeitschrift für Pädagogik, I 8 (I), 307-3 I I.

Trier, U. P. (1976). Schulreform: Wege und Perspektiven. In: K. Widmer (Hg.), Bildungsplanung und Schulreform (S. 73-9I). Frauenfeld: Huber.

Trier, U. P. (I979). SIPRI: Ein Anliegen aller Betroffenen. Schweizerische Lebrerzeitung, 44 (Sondernummer), I 579-I 58 I.

Trier, U. P. (1978). Zur Einleitung: Über die Schwierigkeit, mit Wissenschaft den Unterricht zu verbessern. In: K. Aregger, U. P. Lattmann und U. P. Trier (Hg.), Lebrerbildung und Unterricht (S. 2 I-24). Bern: Haupt.

Tyler, R. W. (1949). Basic Principles of Curriculum and Instruction. Chicago: The University of Chicago Press.

Vernetz, H. (1968). Koordination und Lehrplanreform. Schweizer Schule, 55 (I4-1 5), s60-56I. 
Waterkamp, D. (1976). Bericht. CERI/IPN-Seminar über «Bedingungen und Modelle der Curriculuminnovation» vom 28. September bis I I. Oktober 1975 in Schliersee/Obb. Bildung und Erziehung, 29, 333-338.

Weniger, E. (1975). Theorie der Bildungsinhalte und des Lehrplans (1930/1952). In: E. Weniger, B. Schonig und B. Schwenk (Hg.), Ausgewäblte Schriften zur geisteswissenschaftlichen Pädagogik (S. 199-294). Weinheim: Beltz.

Widmaier, H. P. (1968). Zur Strategie der Bildungspolitik. Bern: Stämpfli.

Widmer, K. (1976). Vorwort: Bildungsplanung und Schulreform zwischen Euphorie und Resignation. In: K. Widmer (Hg.), Bildungsplanung und Schulreform (S. 7-2I). Frauenfeld: Huber.

\section{Sekundärliteratur}

Adler, J. und Künzli, R. (1997). Lehrplanarbeit in der Schweiz. Eine Darstellung von Verfabren und Prozessen in sieben Kantonen (NFP 33: Wirksamkeit unserer Bildungssysteme «Von der Lehrplanung zur Lehrplanorganisation»). Aarau.

Amann, K. (I 994). «Guck mal, Du Experte». Wissenschaftliche Expertise unter ethnographischer Beobachtung und wissenssoziologischer Rekonstruktion. In: R. Hitzler, A. Honer und C. Maeder (Hg.), Expertenwissen. Die institutionalisierte Kompetenz zur Konstruktion von Wirklichkeit (S. 32-43). Opladen: Westdeutscher Verlag.

Ash, M. G. (2002). Wissenschaft und Politik als Ressourcen für einander. In: R. vom Bruch (Hg.), Wissenschaften und Wissenschaftspolitik. Bestandsaufnabmen zu Formationen, Brüchen und Kontinuitäten im Deutschland des 20. Jabrhunderts (S. 32-5 I). Stuttgart: Steiner.

Bähr, K. (1999). Lebrplanarbeit - Strukturen, Erwartungen, Perspektiven. Umsetzungsbericht. Aarau: Schweizerische Koordinationsstelle für Bildungsforschung.

Bain, D. und Roller, S. (Hg.). (200I). L'épopée des centres de recherche en éducation en Suisse, 1960-2000 / Die Geschichte der Bildungsforschungsstellen in der Schweiz, 1960-2000. Neuchâtel: IRDP.

Baur, N., Besio, C., Norkus, M. und Petschick, G. (2016). Wissenschaft als Mehrebenen-Phänomen. Der Makro-Meso-Mikro-Link in der Wissenschaft. In: N. Baur, C. Besio, M. Norkus und G. Petschick (Hg.), Wissen-Organisation - Forschungspraxis. Der Makro-Meso-Mikro-Link in der Wissenschaft (S. I 3-43). Weinheim: Beltz Juventa.

Beck, U. und Bonss, W. (1985). Was will und wozu dient «Verwendungsforschung»? In H.-W. Franz (Hg.), 22. Deutscher Soziologentag I984. Sektions- und Ad-boc-Gruppen (S. 610-6I3). Wiesbaden: VS Verlag für Sozialwissenschaften.

Behm, B., Drope, T., Glaser, E. und Reh, S. (2017). Wissen machen. In: S. Reh, E. Glaser, B. Behm und T. Drope (Hg.), Wissen machen. Beiträge zu einer 
Geschichte erziebungswissenschaftlichen Wissens in Deutschland zwischen I945 und I990 (S. 7-15). Weinheim: Beltz Juventa.

Berthoud, C. (2016). Le cycle d'orientation genevois. Une école secondaire pour démocratiser l'accès à la culture, 1927-1977. Gollion: Infolio.

Bosche, A. (2013). Schulreformen steuern. Die Einfübrung neuer Lebrmittel und Schulfächer an der Volksschule. Bern: hep.

Bosche, A. (20I6). The back office of school reform: educational planning units in German-speaking Switzerland (I960s and I970s). Paedagogica Historica, $52(4), 380-394$.

Böschen, S. (2010). Wissenschaft: Epistemisches Niemandsland? In A. Engelhardt und L. Kajetzke (Hg.), Handbuch Wissensgesellschaft. Theorien, Themen und Probleme (S. I 50-I60). Bielefeld: transcript.

Bourdieu, P. (1993). Über einige Eigenschaften von Feldern. In: Soziologische Fragen (S. I07-I I 4). Frankfurt am Main: Suhrkamp Taschenbuch.

Bourdieu, P. (1997). Les usages sociaux de la science. Pour une sociologie clinique du champ scientifique. Paris: INRA Editions.

Bradley, J. H., Paul, R. und Seeman, E. (2006). Analyzing the structure of expert knowledge. Information E Management, 43 (I), 77-91, https://doi.orı g/I0.10I6/j.im.2004.I I.009

Brandstetter, T. (20I 2). Wissenschaftsgeschichte. In: S. Maasen, M. Kaiser, M. Reinhart und B. Sutter (Hg.), Handbuch Wissenschaftssoziologie (S. 24I-249). Wiesbaden: Springer Fachmedien.

Braun-Thürmann, H. (2010). Wandel der Wissensproduktion. In: D. Simon, A. Knie und S. Hornbostel (Hg.), Handbuch Wissenschaftspolitik (S. 7I-88). Wiesbaden: VS Verlag für Sozialwissenschaften.

Breidbach, O. (2007). Wissenschaftsgeschichte. In: R. Schützeichel (Hg.), Handbuch Wissenssoziologie und Wissensforschung (S. 8 I 4-835). Konstanz: UVK Verlagsgesellschaft.

Brosziewski, A. (2016). Aufstieg und Fall der Unterrichtsexperten oder: Wozu sich Professionelle von Experten unterscheiden. In: J. Raab und R. Keller (Hg.), Wissensforschung - Forschungswissen. Beiträge und Debatten zum I. Sektionskongress der Wissenssoziologie. Weinheim: Beltz Juventa.

Bürgi, R. (2017). Die OECD und die Bildungsplanung der freien Welt. Denkstile und Netzwerke einer internationalen Bildungsexpertise. Leverkusen: Budrich, Barbara.

Burke, P. (20I4). Die Explosion des Wissens. Von der Encyclopédie bis Wikipedia. Berlin: Wagenbach.

Carrier, M. (20I4). Wissenschaftliche Expertise. Bedingungen ihrer Verlässlichkeit und Vertrauenswürdigkeit. In: A. Bora, A. Henkel und C. Reinhardt (Hg.), Wissensregulierung und Regulierungswissen (S. I 57-I74). Weilerswist: Velbrück Wissenschaft.

Criblez, L. (1995). Zwischen Pädagogik und Politik. Bildung und Erziehung in der deutschsprachigen Schweiz zwischen Krise und Krieg (1930-1945). Bern: Lang. 
Criblez, L. (200Ia). Bildungsexpansion durch Systemdifferenzierung - am Beispiel der Sekundarstufe II in den I960er- und I970er-Jahren. Schweizerische Zeitschrift für Bildungswissenschaften, 23 (I), 95-I I 8.

Criblez, L. (200Ib). Editorial: Die Bildungsexpansion in der Schweiz der 1960er- und I970er-Jahre. Schweizerische Zeitschrift für Bildungswissenschaften, 23 (I), $5-\mathrm{I} 2$.

Criblez, L. (200Ic). Promotion - Ein Weg in die Erziehungswissenschaften? Actes du congrès 2000 de la Société suisse pour la recherche en éducation, CD-ROM.

Criblez, L. (2002a). Fragil und unstet - Zur Entwicklung der Erziehungswissenschaften an den Universitäten der deutschsprachigen Schweiz. In: R. Hofstetter und B. Schneuwly (Hg.), Erziebungswissenschaft(en). 19.-20. Jabrbundert. Zwischen Profession und Disziplin (S. 425-453). Bern: Lang.

Criblez, L. (2002b). Wozu Pädagogik? Zum Funktionswandel der Pädagogik in der Lehrerbildung. Beiträge zur Lebrerbildung, 20 (3), 300-3 I 8.

Criblez, L. (2007a). Bildungsföderalismus und Schulkoordination: konfligierende Prinzipien der Schulpolitik in der Schweiz. In: R. Casale und R. Horlacher (Hg.), Bildung und Öffentlichkeit. Jürgen Oelkers zum 60. Geburtstag (S. 262-276). Weinheim und Basel: Beltz.

Criblez, L. (2007b). Die Neukonstituierung der Bildungsforschung in der Schweiz Ende der i960er und anfangs der I970er Jahre - und deren Konsequenzen. In: C. Crotti und F. Osterwalder (Hg.), Pädagogik und Politik. Historische und aktuelle Perspektiven. Festschrift für Fritz Osterwalder (S. 205-228). Bern: Haupt.

Criblez, L. (2008). Bildungsforschung und Bildungspolitik oder: von überdauernden Problemen der Grenzziehung. Eine Replik auf Walter Herzog. Schweizerische Zeitschrift für Bildungswissenschaften, 30 (I), I 53-166.

Criblez, L. (201 5a). Forschung im Bildungsbereich: Aufgabe der akademischen Disziplin, der Bildungsplanungsstellen und der Pädagogischen Hochschulen. In: E. Glaser (Hg.), Unscharfe Grenzen - eine Disziplin im Dialog. Pädagogik, Erziehungswissenschaft, Bildungswissenschaft, Empirische Bildungsforschung (S. 5 I-70). Bad Heilbrunn: Klinkhardt.

Criblez, L. (20 I 5b, Oktober). Bildungsexpansion - Phänomen und Kontexte. Kolloquium HBS, Institut für Erziehungswissenschaft der Universität Zürich.

Criblez, L. (20 I6a). Die Transformation von Staatlichkeit in der Schweiz I960-1990 aus bildungshistorischer Perspektive. In: C. Rothen, L. Criblez und T. Ruoss (Hg.), Staatlichkeit in der Schweiz. Regieren und verwalten vor der neoliberalen Wende (S. 385-40I). Zürich: Chronos.

Criblez, L. (2016b). Erziehungswissenschaft in der Schweiz - aktuelle Situation und Entwicklungstendenzen. Zeitschrift für Erziehungswissenschaft, 27 (I), 69-83.

Criblez, L., Oelkers, J., Reusser, K., Berner, E., Halbheer, U. und Huber, C. (2009). Bildungsstandards. Zug: Klett und Balmer.

Deplazes, D. (2020). «Balance of mind (...) seems more necessary than the promotion of teaching machines»- Technology in Swiss Schools in the I 960 . IJHE 
Bildungsgeschichte - International Journal for the Historiography of Education $\mathrm{I} \circ(\mathrm{I}), 42-63$.

Drerup, H. (1989). Probleme ausserwissenschaftlicher Verwendbarkeit von Erzien hungswissenschaft. Zum Einfluss von Erziehungswissenschaft im politischadministrativen Bereich. In: U. Bracht, E. König und P. Zedler (Hg.), Rezeption und Verwendung erziehungswissenschaftlichen Wissens in pädagogischen Handlungs- und Entscheidungsfeldern (S. I43-165). Weinheim: Deutscher Studien-Verlag.

Drews, K., Hubrich, A.-K., Ludwig, S., Renker, S. und Schütt, F. (Hg.). (2019). Die Frage in den Geisteswissenschaften. Herausforderungen, Praktiken und Reflexionen. Berlin: Frank \& Timme.

Düring, M., Eumann, U., Stark, M. und Keyserlingk, L. von (Hg.). (2016). Handbuch Historische Netzwerkforschung. Grundlagen und Anwendungen. Berlin: LIT.

Düring, M. und Keyserlingk, L. von. (2015). Netzwerkanalyse in den Geschichtswissenschaften. Historische Netzwerkanalyse als Methode für die Erforschung von historischen Prozessen. In: R. Schützeichel und S. Jordan (Hg.), Prozesse (S. 337-350). Wiesbaden: Springer Fachmedien.

Fabian, S. (2016). Boom in der Krise. Konsum, Tourismus, Autofabren in Westdeutschland und Großbritannien 1970-1990. Göttingen: Wallstein.

Fangerau, H. (2009). Der Austausch von Wissen und die rekonstruktive Visualisierung formeller und informeller Denkkollektive. In: H. Fangerau und T. Halling (Hg.), Netzwerke. Allgemeine Theorie oder Universalmetapher in den Wissenschaften? Ein transdisziplinärer Überblick (S. 2 I 5-246). Bielefeld: Transcript.

Fleck, L. (1935). Entstehung und Entwicklung einer wissenschaftlichen Tatsache. Einfübrung in die Lehre vom Denkstil und Denkkollektiv. Basel: Benno Schwabe.

Frey, A. (20I7). Informationsgespräch zur FAL. Entstehung, Empirie und Weiterentwicklung der Curriculumforschung.

Fuhse, J. (2019). Netzwerke im Feld der Politik. In: J. Fuhse und K. Krenn (Hg.), Netzwerke in gesellschaftlichen Feldern (S. 8 I-I09). Wiesbaden: Springer VS, www.academia.edu/37360 I22/Netzwerke_im_Feld_der_Politik

Gees, T. (2016). Viel Diskurs - wenig Steuerung. Schweizer Wissenschaftspolitik in der Mehrebenenrealität. In: L. Criblez, C. Rothen und T. Ruoss (Hg.), Staatlichkeit in der Schweiz. Regieren und verwalten vor der neoliberalen Wende (S. 3 I 7-340). Zürich: Chronos.

Geiss, M. (2018). Zeitdiagnostik und die Altlasten der Bildungsexpansion. Zeitschrift für Pädagogik, 64 (I), 42-50.

Gilcher-Holtey, I., Eckert, R., François, E., Klessmann, C. und Ruchniewicz, K. (2006). Debatte. Die I 970er-Jahre in Geschichte und Gegenwart. Zeithistorische Forschungen / Studies in Contemporary History, (3), 422-438.

Goffman, E. (I99I). Asyle. Über die soziale Situation psychiatrischer Patienten und anderer Insassen. Frankfurt am Main: Suhrkamp. 
Göldi, S. (20I I). Von der bloomschen Taxonomy zu aktuellen Bildungsstandards. Zur Entstehungs- und Rezeptionsgeschichte eines pädagogischen Bestsellers. Bern: hep.

Gonon, P. (1998). Das internationale Argument in der Bildungsreform. Die Rolle internationaler Bezüge in den bildungspolitischen Debatten zur schweizerischen Berufsbildung und zur englischen Reform der Sekundarstufe II. Bern: Lang.

Graf, R. (20I4). Öl und Souveränität. Petroknowledge und Energiepolitik in den USA und Westeuropa in den I970er Jahren. Berlin: de Gruyter Oldenbourg.

Gretler, A. (1979a). Löst sich die Freiburger Arbeitsgruppe für Lehrplanforschung (FAL) auf? Bildungsforschung und Bildungspraxis, I (I), 95-97.

Gretler, A. (I 979b). Organismes d'enseignement et de recherche. Revue française de pédagogie, 47, 109-1 23.

Gretler, A. (1982). Bemerkungen zum Selbstverständnis der Bildungsforschung in der Schweiz. Bildungsforschung und Bildungspraxis, 4 (2), I I7-I 23.

Gretler, A. (1983). Finanzierung der Curriculumforschung in der Schweiz. In: K. Frey, U. Hameyer und H. Haft (Hg.), Handbuch der Curriculumforschung (S. 747-750). Weinheim und Basel: Beltz.

Gretler, A. (2000). Die schweizerische Bildungsforschung der Nachkriegszeit im Spiegel ihrer Institutionen und ihrer Themen - von der Geschichte zu aktuellen Fragestellungen. Scbweizerische Zeitschrift für Bildungswissenschaften, 22 (I), I I I-I 44.

Gretler, A. (200I). Entwicklungen der universitären und ausseruniversitären Bildungsforschung - Gewichtsverschiebungen in der zweiten Hälfte des 20. Jahrhunderts. In: D. Bain, J. Brun, D. Hexel und J. Weiss (Hg.), Die Geschichte der Bildungsforschungsstellen in der Schweiz I960-2000 (S. 3 I I323). Lausanne: IRDP, Neuchâtel.

Grossenbacher, S. und Oggenfuss, C. (2015). «Information Bildungsforschung». 40 Jabre Forschungsdokumentation der SKBF (Nr. I s). Aarau: SKBF.

Gruber, H. und Rehrl, M. (2009). Netzwerkforschung. In: R. Tippelt und B. Schmidt (Hg.), Handbuch Bildungsforschung (S. 967-98 I). Wiesbaden: Springer VS.

Gruber, K. H. (1992). Anzeichen konvergierender Trends der europäischen Schulund Curriculumentwicklung. In: Schweizerische Konferenz der kantonalen Erziehungsdirektoren, Bund-Länder-Kommission für Bildungsplanung und Forschungsförderung und Bundesministerium für Unterricht und Kunst (Hg.), Lernen für Europa. Neue Lebens- und Lernformen in der Schule. OECD/CERI-Seminar Stift Geras I99I (S. 2 I-29). Bonn: Köllen.

Haas, P. (I992). Epistemic communities and international policy coordination Introduction. International Organization, 46 (I), I-35.

Haas, P. (200I). Policy Knowledge: Epistemic Communities. In: P. B. Baltes und Smelser (Hg.), International Encyclopedia of the Social E Behavioral Sciences (S. I I 578-I I 586). Oxford: Pergamon, www.sciencedirect.com/science/article/ pii/Boo80430767045265

Haas, P. (20I I). Epistemic Communities. In: International Encyclopedia of Political Science (S. I I 578-I I 586). California: Elsevier Ltd. 
Hafner, U. (2015). Vom Wissenschaftsrat zum Innovationsrat. Die historische Entwicklung des Schweizerischen Wissenschaftsrates in der Aussensicht (Schrift Nr. 4). Bern: Schweizerischer Wissenschafts- und Innovationsrat SWIR.

Halling, T. und Fangerau, H. (2009). Netzwerke - Eine allgemeine Theorie oder die Anwendung einer Universalmetapher in den Wissenschaften? In T. Halling und H. Fangerau (Hg.), Netzwerke. Allgemeine Theorie oder Universalmetapher in den Wissenschaften? Ein transdisziplinärer Überblick (S. 267285 ). Bielefeld: Transcript.

Hameyer, U. (1982). Die Ordnung der Zeit in den Erwartungen an die Curriculumforschung. In: Zeit für Veränderungen (S. 79-1 55). Opladen: Leske Verlag + Budrich, https://doi.org/I0.1007/978-3-322-84185-8_5

Hameyer, U. (1983). Systematisierung von Curriculumtheorien. In: U. Hameyer, K. Frey und H. Haft (Hg.), Handbuch der Curriculumforschung (S. 54-100). Weinheim und Basel: Beltz.

Hameyer, U. (I992). Stand der Curriculumforschung - Bilanz eines Jahrzehnts. Zeitschrift für Lernforschung, 20 (3), 209-232.

Hameyer, U., Aregger, K. und Frey, K. (Hg.). (1976). Bedingungen und Modelle der Curriculuminnovation. Bericht über das CERI/IPN-Seminar vom 28. September bis I I. Oktober I975 in Schliersee. Weinheim: Beltz.

Hameyer, U., Frey, K. und Haft, H. (Hg.). (1983). Handbuch der Curriculumforschung. Übersichten zur Forschung. Weinheim: Beltz.

Heinze, T. (201 2). Netzwerke der Wissenschaft. In: S. Maasen, M. Kaiser, M. Reinhart und B. Sutter (Hg.), Handbuch Wissenschaftssoziologie (S. 19I-20I). Wiesbaden: Springer Fachmedien, https://doi.org/I0.1007/978-3-53 I-I 891 8S_IS

Hoffmann, D. (2005). Heinrich Roth. In: Neue Deutsche Biographie (Bd. 22, S. I I 3 f.), www.deutsche-biographie.de/pnd i 8603 I 24 .html\#ndbcontent Hoffmann-Ocon, A. (2013). Netzwerke in bildungshistorischer Perspektive Versuch einer disziplintheoretischen Annäherung. In: H.-U. Grunder, A. Hoffmann-Ocon und P. Metz (Hg.), Netzwerke in bildungshistorischer Perspektive (S. 23-32). Bad Heilbrunn: Klinkhardt.

Hofstetter, R., Ratcliff, M. und Schneuwly, B. (20I2). Cent ans de vie I9I2-20I2. La Faculté de psychologie et des sciences de l'éducation héritière de l'Institut Rousseau et de l'ère piagétienne. Geneve: Georg.

Höhener, L. (2018). Zur latenten Kontinuität der Curriculumdebatte in den I980er-Jahren - eine Spurensuche in der Schweiz. In: L. Criblez und A. Hoffmann-Ocon (Hg.), Schwerpunkt Scheinbarer Stillstand. Pädagogische Diskurse und Entwicklungen in den Achtzigerjabren (Bd. 23, S. I 19-I38). Bad Heilbrunn: Julius Klinkhardt.

Höhener, L. und Criblez, L. (20I 8). Wissenstransfer im Kontext der Schweizer Curriculumdiskussionen der I970er-Jahre. Schweizerische Zeitschrift für Bildungswissenschaften, 40 (I), 87-I IO. 
Holzer, B. (2009). Netzwerktheorie. In: G. Kneer und M. Schroer (Hg.), Handbuch Soziologische Theorien (S. 253-275). Wiesbaden: VS Verlag für Sozialwissenschaften, https://doi.org/I0.1007/978-3-53 I-9I600-2_I3

Honegger, C. (2007). Aufbruch in die Planungseuphorie. In: C. Honegger, H.-U. Jost, S. Burren und P. Jurt (Hg.), Konkurrierende Deutungen des Sozialen. Geschichts-, Sozial-und Wirtschaftswissenschaften im Spannungsfeld von Politik und Wissenschaft (S. 107-I 52). Zürich: Chronos.

Hopmann, S. und Künzli, R. (2015). Spielräume der Lebrplanarbeit. Grundzüge einer Theorie der Lebrplanung, http://bildungswissenschaft.univie.ac.at/fileadmin/user_upload/inst_bildungswissenschaft/Spielr_ume_der_Lehrplanarbeit.pdf, abgerufen am 24. 9. 2015 .

Horlacher, R. und De Vincenti, A. (20I4). From Rationalist Autonomy to Scientific Empiricism. A History of Curriculum in Switzerland. In: W. F. Pinar (Hg.), International Handbook of Curriculum Research (S. 476-492). New York: Routledge.

Horn, K.-P. (20I4). Pädagogik/Erziehungswissenschaft der Gegenwart. In: R. Fatke und J. Oelkers (Hg.), Das Selbstverständnis der Erziehungswissenschaft. Geschichte und Gegenwart (S. I4-32). Weinheim: Beltz Juventa.

Hoyningen-Huenem, P. (1989). Wissenschaftsentwicklung und Wirklichkeit in der Theorie Thomas S. Kuhns. Deutsche Zeitschrift für Philosophie, 37 (6), 508.

Huber, C. (20I7). Lebrerinnen- und Lebrerbildungsregion Zentralschweiz. Bildungspolitische Entwicklungen seit Ende der I95oer Jabre. Wien, Zürich: LIT.

Hübinger, G. (2013). Wissenschaften, Zeitdiagnosen und politisches Ordnungsdenken. In: G. Hübinger (Hg.), Europäische Wissenschaftskulturen und politische Ordnungen in der Moderne (I890-1970) (S. I-28). München: Oldenbourg.

Ingenkamp, K. (I992). Die Anfänge der Arbeitsgruppe für empirische pädagogische Forschung, AEPF, 1965-1969. Zeitschrift zu Theorie und Praxis erziehungswissenschaftlicher Forschung, 6 ( I), I09-I I 7.

Jansen, D. (2003). Einfübrung in die Netzwerkanalyse. Grundlagen, Methoden, Forschungsbeispiele. Opladen: Leske + Budrich.

Jarausch, K. H. (2006). Krise oder Aufbruch? Historische Annäherungen an die I 970 r-Jahre. Zeithistorische Forschungen / Studies in Contemporary History, (3), 334-34I.

Jasanoff, S. (1994). The Fifth Branch. Science Advisers as Policymakers. Cambridge, MA: Harvard University Press.

Jenzer, C. (1995). Entwicklung des Schulwesens in der Schweiz. In: Schweizerische Konferenz der kantonalen Erziehungsdirektoren, Bund-Länder-Kommission für Bildungsplanung und Forschungsförderung und Bundesministerium für Unterricht und Kunst (Hg.), Innovationen im Bildungswesen als übernationale Aufgabe (S. 35-40). Bonn: Köllen.

Keiner, E. (2002). Rezeption und Verwendung erziehungswissenschaftlichen Wissens. In: L. Wigger (Hg.), Beibeft zur Zeitschrift für Pädagogik (S. 24 I-249).

Keller, R. und Proferl, A. (20I6). Wissenskulturen als Forschungsgegenstand. In: J. Raab und R. Keller (Hg.), Wissensforschung - Forschungswissen. Beiträge 
und Debatten zum I. Sektionskongress der Wissenssoziologie (S. 740-75 I). Weinheim: Beltz Juventa.

Ketelhut, K. (20I3). Netzwerke(r) in der historischen Bildungsforschung: der Reformpädagoge Berthold Otto. In: H.-U. Grunder, A. Hoffmann-Ocon und P. Metz (Hg.), Netzwerke in bildungshistorischer Perspektive (S. I I I-I 20). Bad Heilbrunn: Klinkhardt.

Knorr Cetina, K. (I999). Epistemic cultures: how the sciences make knowledge. Choice Reviews Online, 37 (02), 329, https://doi.org/ı0.5860/ CHOICE.37-0887

König, E. und Zedler, P. (1998). Die wissenschaftstheoretische Diskussion um die «richtige Wissenschaft». In: E. König und P. Zedler (Hg.), Theorien der Erziehungswissenschaft. Einfübrung in Grundlagen, Methoden und praktische Konsequenzen (S. 206-240). Weinheim: Beltz.

Kuhn, T. (1967). Die Struktur wissenschaftlicher Revolutionen. Frankfurt am Main: Suhrkamp.

Künzli, R. (1999). Lebrplanarbeit. Über den Nutzen von Lebrplänen für die Schule und ibre Entwicklung. Chur, Zürich: Rüegger.

Künzli, R. (2006a). Kantonale Lehrplanpolitik in der Schweiz. In: L. Criblez, P. Gautschi, P. Hirt Monico und H. Messner (Hg.), Lehrpläne und Bildungsstandards. Was Schülerinnen und Schüler lernen sollen. Festschrift zum 65. Geburtstag von Rudolf Künzli (S. 6I-78). Bern: hep.

Künzli, R. (2006b). Standards statt Lehrpläne - zurück zu den Bildungsinhalten? In L. Criblez, P. Gautschi, P. Hirt Monico und H. Messner (Hg.), Lebrpläne und Bildungsstandards. Was Schülerinnen und Schüler lernen sollen. Festschrift zum 65. Geburtstag von Rudolf Künzli (S. 83-102). Bern: hep.

Künzli, R. (20I I). Der Lehrplan als Auftrag der Gesellschaft an die Schule. In: L. Criblez, B. Müller und J. Oelkers (Hg.), Die Volksschule - zwischen Innovationsdruck und Reformkritik (S. 200-2 I6). Zürich: Verlag Neue Zürcher Zeitung.

Künzli, R., Bähr, K., Fries, A.-V., Ghisla, G., Rosenmund, M. und Seliner-Müller, G. (1999). Lehrplanarbeit. Über den Nutzen von Lebrplänen für die Schule und ibre Entwicklung. Chur, Zürich: Rüegger.

Künzli, R. und Frey, K. (1977). Fachdisziplinen in der Bildung - Disziplinierung oder Befreiung durch Wissenschaft? Zeitschrift für Pädagogik. Beibeft, I (I 3), 273-280.

Künzli, R., Fries, A.-V., Hürlimann, W. und Rosenmund, M. (20I3). Der LehrplanProgramm der Schule. Weinheim: Beltz Juventa.

Kussau, J. und Oertel, L. (200I). Bildungsexpansion, Reform der Sekundarstufe I und Pädagogische Arbeitsstellen. Schweizerische Zeitschrift für Bildungswissenschaften, 23 (I), I 37-163.

Leemann, R. J. und Christian, I. (20I I). Zum Zusammenhang von Geschlechterungleichheiten in Bildung, Beruf und Karriere: Ein Ausblick. In: A. Hadjar (Hg.), Geschlechtsspezifische Bildungsungleichbeiten (S. 4I7-440). Wiesbaden: VS Verlag. 
Lehmann, L. (2013). Zwang zur freiwilligen Zusammenarbeit. Steuerungsinstrumente und interkantonale Governance in der schweizerischen Lebrerinnen-und Lebrerbildung. Bern: hep.

Lehmann, L. und Imlig, F. (2018). Folgeprobleme. In: F. Imlig, L. Lehmann und K. Manz (Hg.), Schule und Reform (S. I-I6). Wiesbaden: VS Verlag.

Lengwiler, M. (2010). Konjunkturen und Krisen in der Verwissenschaftlichung der Sozialpolitik des 20. Jahrhunderts. Archiv für Sozialgeschichte, 50, 47-68.

Luhmann, N. (2015). Die Gesellschaft der Gesellschaft, Bd. 2. Frankfurt am Main: Suhrkamp.

Manz, K. (20I I). "Schulkoordination ja-aber nicht so!». Die Anfänge der schweizerischen Schulkoordination (1960-1985). Bern: hep.

Nagel, M. (2009). Wissenstransfernetzwerke. Eine netzwerkanalytische Bewertung der Effektivität von intraorganisationalen Wissenstransfernetzwerken. In: V. Schneider, F. Janning, P. Leifeld und T. Malang (Hg.), Politiknetzwerke (S. 353-367). Wiesbaden: VS Verlag für Sozialwissenschaften, https://doi. org/I0.1007/978-3-53 I-91883-9_i6

Nezel, I. und Ghisla, G. (1977). Struktur und Curriculum. Theorie und Verfabren zur Konstruktion und Analyse curricularer Wissensstrukturen. Weinheim: Beltz.

Oelkers, J. (2007). Einige Bemerkungen zum Verhältnis von Erziehungswissenschaft und Bildungspolitik. In: U. Herrmann (Hg.), In der Pädagogik etwas bewegen. Impulse für Bildungspolitik und Schulentwicklung (S. 62-69). Weinheim: Beltz.

Patry, J.-L. und Gretler, A. (1992). Bildungsforschung in der Schweiz I 970 bis I990: Interdisziplinarität und Forschung-Praxis-Bezug. Zeitschrift zu Theorie und Praxis erziehungswissenschaftlicher Forschung, 6 (I), 33-7 I.

Plotke, H. (1979). Schweizerisches Schulrecht. Bern: Haupt.

Plumpe, W. (2009). Der Abschied von der Gesellschaft: Sozioökonomischer Strukturwandel und die Paradoxien des Wissens in den i $960 e r$ bis I $980 e r$ Jahren. In: J. Fried und M. Stolleis (Hg.), Wissenskulturen. Über die Erzengung und Weitergabe von Wissen (S. 103-I24). Frankfurt am Main, New York: Campus.

Radtke, F.-O. (1996). Wissen und Können. Die Rolle der Erziehungswissenschaft in der Erziebung. Wiesbaden: VS Verlag für Sozialwissenschaften.

Raphael, L. (1996). Die Verwissenschaftlichung des Sozialen als methodische und konzeptionelle Herausforderung für eine Sozialgeschichte des 20. Jahrhunderts. Geschichte und Gesellschaft, 22, 165-193.

Raphael, L. (20I3). Zwischen Sozialaufklärung und radikalem Ordnungsdenken. In: G. Hübinger (Hg.), Europäische Wissenschaftskulturen und politische Ordnungen in der Moderne (1890-1970) (S. 29-50). München: Oldenbourg.

Reichenbach, R. (2017). Theoriefreie Bildungsforschung. In: T. Rucker (Hg.), Erkenntnisfortschritt (in) der Erziebungswissenschaft. Lernt die Disziplin?

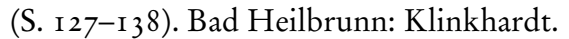

Reichmann, W. und Knorr Cetina, K. (2016). Wissenskulturen. Wissenschaftliche Praxis und gesellschaftliche Ordnung. In: N. Baur, C. Besio, M. Norkus und 
G. Petschick (Hg.), Wissen - Organisation - Forschungspraxis. Der MakroMeso-Mikro-Link in der Wissenschaft (S. 46-70). Weinheim: Beltz Juventa.

Reitmayer, M. und Marx, C. (20I0). Netzwerkansätze in der Geschichtswissenschaft. In: C. Stegbauer und R. Häußling (Hg.), Handbuch Netzwerkforschung (S. 869-880). Wiesbaden: VS Verlag für Sozialwissenschaften.

Rheinberger, H.-J. (2007). Historische Epistemologie zur Einführung. Hamburg: Junius.

Richards, R. J. und Daston, L. (Hg.). (2016). Kubn's Structure of Scientific Revolutions at Fifty. Reflections on a Science Classic. Chicago: University of Chicago Press.

Rieger-Ladich, M. (2009). Pierre Bourdieus Theorie des wissenschaftlichen Feldes: Ein Reflexionsangebot an die Erziehungswissenschaft. In: B. Friebertshäuser, M. Rieger-Ladich und L. Wigger (Hg.), Reflexive Erziehungswissenschaft. Forschungsperspektiven im Anschluss an Pierre Bourdieu (S. I55-174). Wiesbaden: VS Verlag für Sozialwissenschaften.

Rieger-Ladich, M. (2019). Archivieren und Speichern. Das Gedächtnis der Disziplin als Politikum. In: M. Rieger-Ladich, A. Rohstock und K. Amos (Hg.), Erinnern, Umschreiben, Vergessen. Die Stiftung des disziplinären Gedächtnisses als soziale Praxis (S. 17-49). Weilerswist: Velbrück Wissenschaft.

Roelcke, V. (2010). Auf der Suche nach der Politik in der Wissensproduktion: Plädoyer für eine historisch-politische Epistemologie. Berichte zur Wissenschaftsgeschichte, 33 (2), 176-192.

Rothen, C. (2016). Educational research within the administration: a booming business in the French-speaking part of Switzerland (1950-I980). Paedagogica Historica, I-I 3 .

Rüegg, S., Dunkel, S. und Schuppli, C. (2009). Von der Idee zum Institut. ULEFeine Erfolgsgeschichte (Sonderdruck). Oberentfelden: Sauerländer Verlage.

Saldern, M. von (I992). Zur Geschichte der AEPF. In: K. Ingenkamp, H. Pettillon, S. R. Jäger und B. Wolf (Hg.), Empirische Pädagogik, 1970-1990. Eine Bestandsaufnabme der Forschung in der Bundesrepublik Deutschland (Bd. 2, S. 684-705). Weinheim: Deutscher Studien Verlag.

Saldern, M. von (20I0). Geschichte der Empirischen Pädagogik/Erziehungswissenschaft in der Bundesrepublik - Offene Fragen. In: C. Ritzi und U. Wiegmann (Hg.), Beobachten - Messen - Experimentieren. Beiträge zur Geschichte der empirischen Pädagogik/Erziehungswissenschaft (S. 305-328). Bad Heilbrunn: Klinkhardt.

Sarasin, P. (20I I). Was ist Wissensgeschichte? Internationales Archiv für Sozialgeschichte der deutschen Literatur (IASL), 36 (I), I 59-I 72.

Sarasin, P. und Kilcher, A. (20 I I). Editorial. In: D. Gugerli, M. Hagner, P. Sarasin und J. Tanner (Hg.), Zirkulationen (S. 7-Io). Zürich: Diaphanes.

Schützeichel, R. (2007a). Laien, Experten, Professionen. In: R. Schützeichel (Hg.), Handbuch Wissenssoziologie und Wissensforschung (Bd. I , S. 546-578). Konstanz: UVK. 
Schützeichel, R. (2007b). Soziologie des wissenschaftlichen Wissens. In: R. Schützeichel (Hg.), Handbuch Wissenssoziologie und Wissensforschung (Bd. I 5 , S. 306-327). Konstanz: UVK Verlagsgesellschaft.

Schwarzmann, R. (1985). Der Schweizerische Nationalfonds zur Förderung der wissenschaftlichen Forschung. Zürich: Schulthess.

Schweizerischer Nationalfonds zur Förderung der wissenschaftlichen Forschung. (1977). 25 Jabre Schweizerischer Nationalfonds zur Förderung der wissenschaftlichen Forschung. Bern: SNF.

Sheldon, G. (2010). Der Schweizer Arbeitsmarkt seit 1920: Langfristige Tendenzen. Die Volkswirtschaft. Das Magazin für Wirtschaftspolitik, 83 (I/2), I 5-I9.

Speich Chassé, D. und Gugerli, D. (20I2). Wissensgeschichte - eine Standortbestimmung. Traverse, (I), $85-\mathrm{I} 00$.

Stettler, N. (1994). Die Zukunft ist errechenbar ... In J.-D. Blanc und C. Luchsinger (Hg.), Achtung: die soer Jahre! Annäherung an eine widersprüchliche Zeit (S. 95-I I 7). Zürich: Chronos.

Stolleis, M. (2009). Wissenskulturen. Über die Erzeugung und Weitergabe von Wissen. Einleitung. In: J. Fried und M. Stolleis (Hg.), Wissenskulturen. Über die Erzengung und Weitergabe von Wissen (S. 7-I I). Frankfurt am Main, New York: Campus.

Strassheim, H. (20I4). Politische Expertise im Wandel. In: S. Kropp und S. Kuhlmann (Hg.), Wissen und Expertise in Politik und Verwaltung (S. 65-86). Opladen, Toronto: Barbara Budrich.

Strittmatter, A. (2003). Hat LEMO den Rahmenlehrplan verpasst? Beiträge zur Lebrerbildung, $2 \mathrm{I}$ (3), 334-34 I.

Szöllösi-Janze, M. (2004). Wissensgesellschaft in Deutschland: Überlegungen zur Neubestimmung der deutschen Zeitgeschichte über Verwissenschaftlichungsprozesse. Geschichte und Gesellschaft, 30 (2), 277-3 I 3 .

Tenorth, H.-E. (1986). Transformationen der Pädagogik. In: Beibeft der Zeitschrift für Pädagogik (Bd. 20, S. 2 I-8 5). Weinheim: Beltz.

Tenorth, H.-E. (I990). Vermessung der Erziehungswissenschaft. Zeitschrift für Pädagogik, 36 (I), I 5-27.

Tenorth, H.-E. (20I4a). Evidenzbasierte Bildungsforschung vs. Pädagogik als Kulturwissenschaft - Über einen neuerlichen Paradigmenstreit in der wissenschaflichen Pädagogik. Education Sciences $\mid$ Education - Research - Innovation, (3), 5-2 I.

Tenorth, H.-E. (20I4b). Politikberatung und Wandel der Expertenrolle oder: Die Expertise der Erziehungswissenschaft. In: R. Fatke und J. Oelkers (Hg.), Das Selbstverständnis der Erziehungswissenschaft. Geschichte und Gegenwart (S. I39-167). Weinheim: Beltz Juventa.

Terhart, E. (200I). Bildungsforschung, Bildungsadministration, Bildungswirklichkeit: eine systematische Annäherung. In: K.-J. Tillmann und W. Vollstädt (Hg.), Politikberatung durch Bildungsforschung. Das Beispiel: Schulentwicklung in Hamburg (S. 17-32). Wiesbaden: VS Verlag für Sozialwissenschaften. 
Tillmann, K.-J. (1991). Erziehungswissenschaft und Bildungspolitik. Erfahrungen aus der jüngsten Reformphase. Zeitschrift für Pädagogik, 37 (6), 955-974.

Tillmann, K.-J. (2008a). Erziehungswissenschaft und Bildungspolitik. Die Deutsche Schule, $\mathrm{I} 00(\mathrm{I}), 3 \mathrm{I}-42$.

Tillmann, K.-J. (2008b). Schulreform - und was die Erziehungswissenschaft dazu sagen kann. Zeitschrift für Pädagogik, 54 (6), 852-868.

Tillmann, K.-J. (2015). Empirische Bildungsforschung als Aufklärung? Die Deutsche Schule, I07 (3), 299-3 I 4 .

Tillmann, K.-J. und Vollstädt, W. (200I). Bildungsforschung im politischen Auftrag: eine Einführung. In: K.-J. Tillmann und W. Vollstädt (Hg.), Politikberatung durch Bildungsforschung (S. 9-16). Wiesbaden: VS Verlag für Sozialwissenschaften.

Trischler, H. (2015). Experten im Fokus: Grobkonturen einer Wissensgeschichte Europas im langen 20. Jahrhundert. In: M. Kintzinger und S. Steckel (Hg.), Akademische Wissenskulturen. Praktiken des Lebrens und Forschens vom Mittelalter bis zur Moderne (Bd. I3, S. 89-1 20). Basel: Schwabe.

Vandendriessche, J. (201 5). Scientists' Expertise as Performance. Between State and Society, I860-1960. London: Pickering \& Chatto.

Vogel, J. (2004). Von der Wissenschafts- zur Wissensgeschichte. Für eine Historisierung der «Wissensgesellschaft». Geschichte und Gesellschaft, 30 (4), 639-660.

Vogel, K., Bers, C., Hild, A., Brauns, J., Stisser, A. und Horn, K. P. (Hg.). (2018). Wendungen und Windungen in der Erziehungswissenschaft. Empirische Studien. Bad Heilbrunn: Julius Klinkhardt.

Vogel, P. (1989). Zur Rekonstruktion pädagogischer Wissensformen. In: D. Benner, P. Zedler und E. König (Hg.), Rekonstruktionen pädagogischer Wissenschaftsgeschichte. Fallstudien, Ansätze, Perspektiven (S. 429-445). Weinheim: Deutscher Studien-Verlag.

Weingart, P. (2005). Die Stunde der Wabrbeit? Zum Verbältnis der Wissenschaft zu Politik, Wirtschaft und Medien in der Wissensgesellschaft. Weilerswist: Velbrück Wissenschaft.

Weingart, P. (2010). Wissenschaftssoziologie. In: D. Simon, A. Knie und S. Hornbostel (Hg.), Handbuch Wissenschaftspolitik (S. I I 8-i 29). Wiesbaden: VS Verlag für Sozialwissenschaften.

Weingart, P., Lentsch, J. und Ash, M. G. (2015). Wissen-Beraten-Entscheiden. Form und Funktion wissenschaftlicher Politikberatung in Deutschland. Weilerswist: Velbrück Wissenschaft.

Weishaupt, H. (199I). Pädagogische Begleitforschung zwischen Bildungspolitik und Bildungsplanung. In: D. Hoffmann und H. Heid (Hg.), Bilanzierungen erziebungswissenschaftlicher Theorieentwicklung. Erfolgskontrolle durch Wissenschaftsforschung (S. I73-200). Weinheim: Deutscher Studien-Verlag.

Willems, H. (20I0). Figurationssoziologie und Netzwerkansätze. In: C. Stegbauer und R. Häussling (Hg.), Handbuch Netzwerkforschung (S. 255-268). Wiesbaden: VS Verlag für Sozialwissenschaften. 
Wingens, M. (1988). Soziologisches Wissen und politische Praxis. Nenere theoretische Entwicklungen der Verwendungsforschung. Frankfurt am Main: Campus.

Zymek, B. (1975). Das Ausland als Argument in der pädagogischen Reformdiskussion. Schulpolitische Selbstrechtfertigung, Auslandspropaganda, internationale Verständigung und Ansätze zu einer vergleichenden Erziehungswissenschaft in der internationalen Berichterstattung deutscher pädagogogischer Zeitschriften, I87I-I952. Ratingen: Aloys Henn. 